


\section{DISCLAIMER}

This report was prepared as an account of work sponsored by an agency of the United States Government. Neither the United States Government nor any agency Thereof, nor any of their employees, makes any warranty, express or implied, or assumes any legal liability or responsibility for the accuracy, completeness, or usefulness of any information, apparatus, product, or process disclosed, or represents that its use would not infringe privately owned rights. Reference herein to any specific commercial product, process, or service by trade name, trademark, manufacturer, or otherwise does not necessarily constitute or imply its endorsement, recommendation, or favoring by the United States Government or any agency thereof. The views and opinions of authors expressed herein do not necessarily state or reflect those of the United States Government or any agency thereof. 


\section{DISCLAIMER}

Portions of this document may be illegible in electronic image products. Images are produced from the best available original document. 
T/D/SNA - ITIT

\section{MASTER}

MOTICE

POMTIONS OF THIS REPORT ARE ILLEGIBLE. $n$

has been renranicad io orr the best available copj to permu the arbanesi possible arift atility. 
NOZZLE AND HOT BLEED PORT
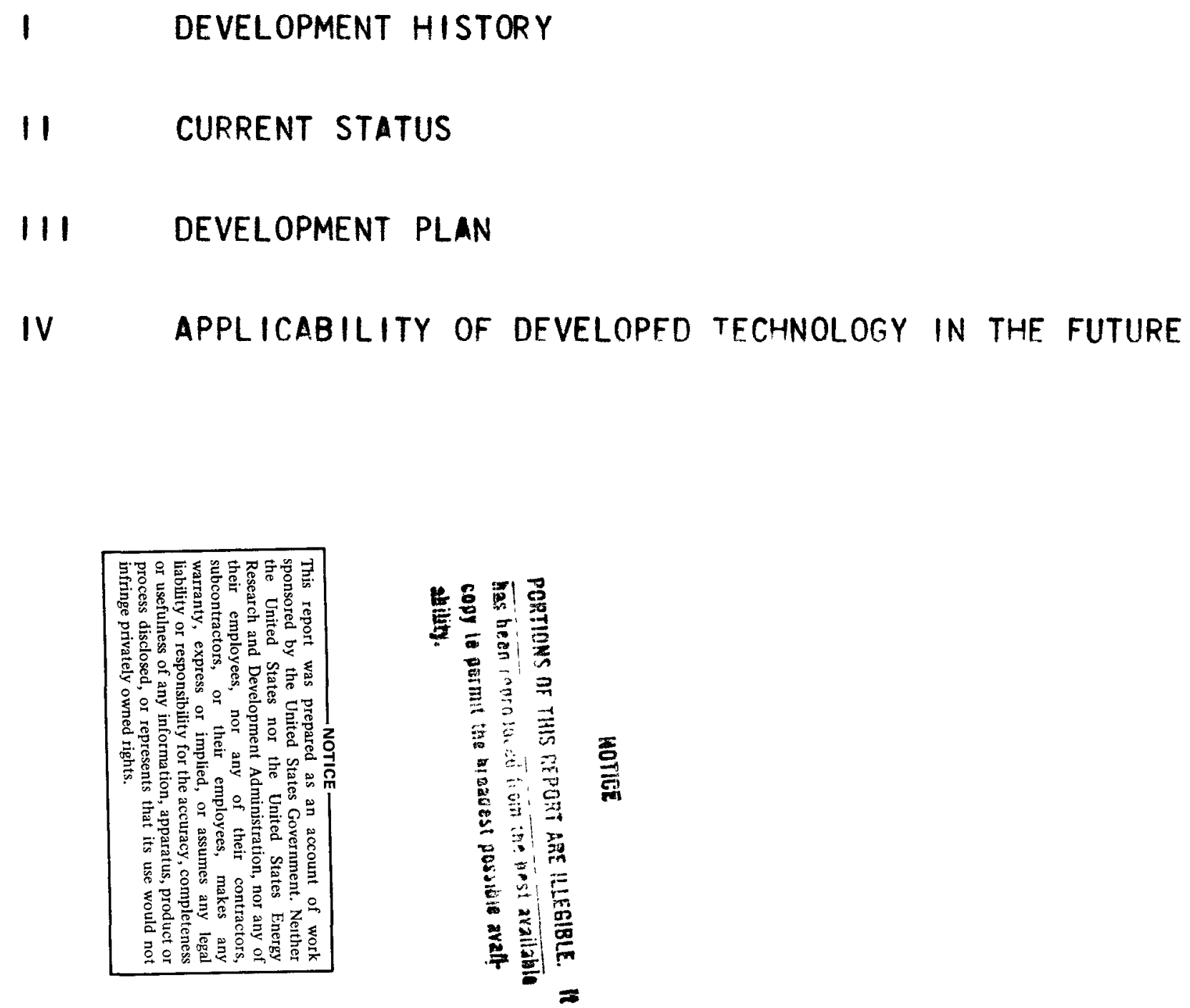
NOZZLE AND HOT BLEED PORT (CON'T)

I NOZZLE DEVELOPMENT HISTORY

A. ORIGINAL NOZZLE CONCEPTS

1. CONVENTIONAL COOLANT TUBE CONSTRUCTION

2. ONE-DIRECT IONAL COOLANT FLOW

3. EXTERNAL STRUCTURAL SHELL - HEAT TREATABLE CAST ALUMINUM

4. WIRE INTERLOCKING THE COOLANT PASSAGES TO THE EXTERNAL SHELL 


\section{NOZZLE AND HOT BLEED PORT CON'T)}

NOZZLE DEVELOPMENT HISTORY

B. PAST PROBLEMS

1. DESIGN-FABR ICATION DIFFICULTIES IMPOSED BY:

A. LONGITUDINAL LOADS

B. HIGH CONVERGENCE RATIO

C. RADIATION HEATING

2. STRUCTURAL FAILURES CAUSED BY:

A. POOR CAST ALUMINUM PROPERTIES

B. TUBE BUNDLE/ALUMINUM JACKET INTFRFACE

3. THERMAL PROBLEMS

A. HEAT TRANSFER COEFFICIENTS NOT VERIFIED

B. HYDROGEN PROPERTIFS NOT STANDARDIZED

C. EXTERNAL JACKET COOLING REOUIREMENTS

D. THROAT COOLANT AREA RELATED TO ENGINE BOOTSTRAP 


\section{NOZZLE AND HOT BLEED PORT (CON'T)}

NOZZLE DEVELOPMENT HISTORY

C. SOLUTIONS TO PROQEMS AND ACHIEVEMENTS

1. ALUMINUM PROPERTIES IMPROVED FROM BRITTLE STATE TO 15\% ELONGATION AND 40 KSI ULTIMATE STRENGTH.UN IFORMITY NOT DETERMINED

2. ELIMINATED STRESS RISES BY MODIFYING THE WIRE INTERLOCK ATTACHMENT

3. MINIMIZED EXTERNAL LEAKAGE

4. CONDUCTED HOT FIRING TESTS AT CHAMBER PRESSURES UP TO 565 PS IA 


\section{NOZZLE AND HOT BLEED PORT}

II CURRENT STATUS

A. EVOLUTION TO NEW DESIGN CONCEPT

1. ELIMINATE POTENTIAL OVERHEATING OF THE EXTERNAL SHELL

2. PRECLUIDE SEPARATION OF THE COOLANT PASSAGES FROM SHELL

3. PRECLUDE POTENTIAL eXTERnAL LEAKAGE

4. ENHANCE FABR ICATION REPRODUCIBILITY

5. SUITABILITY TO MODIFICATION IN EXTERNAL ATTACHMENTS FOR INSTRUMENTATION PURPOSES

6. EXTEND APPLICABILITY TO FUTURE SYSTEMS 


\section{NOZZLE AND HOT BLEED PORT (CON'T)}

II CURRENT STATUS

B. DESIGN DESCRIPTION

1. ONE-DIRECTIONAL COOLANT FLOW THROUGH U-SHAPED BRAZED-IN CHANNELS

2. ONE-PIECE STEEL EXTERNAL SHELL

3. BRAZE JOINT TESTED TO 4000 PSI WITHOUT FAILURE

4. TWO UNITS COMPLETED AND TESTED, THREE UNITS WITH UPGRADED COOLING CAPABILITY IN PROCESS 
NOZZLE AND HOT BLEED PORT (CONT'D)

II CURRENT STATUS

C. CURRENT PROBLEMS

1. DESIGN AND FABRICATION - NONE

2. THERMAL AND COOLING ASPECTS:

UNDEF INED AREAS ARF CIRCUMVENED BY DESIGN CONSERVAT IVE ENOUGH TO COVER ALL CONTINGENCIES

3. TUBE DAMAGE BY PYROTECTIC IGNITERS: CONVERTING TO SPARK IGNITION SYSTEM 


\section{NOZZLE AND HOT BLEED PORT (CON'T)}

III ORIGINAL HOT BLEED PORT CONCFPT

A. PURPOSE :

TO PROVIDE TURB INE DRIVING FLUID

B. MEANS OF ACHIEVEMENT:

MIX HOT CHAMBER PLENUM GAS WITH COLD DILUENT HYDROGEN FROM THE COOLANT PASSAGE 


\section{NOZZLE ANO HOT BLEED PORT}

III ORIGINAL HOT BLEED PORT CONCFPT

C. HOT BLEED PORT PRELIMINARY WORK

1. DES IGNED AND FABRICATED 2.4-IN. DIA UNIT

2. CONDUCTED (18) TESTS ON A TITAN CHAMBER

3. EXPERIENCE GAINED

A. LOCATED HOT SPOTS WITHIN THE ASSEMBLY

B. ELIMINATED OVERHEAT ING BY MODIFYING DILUENT INJECTION

C. ESTABLISHED NEED FOR LARGER BLEFD PORT DIAMETER

D. EVALUATED EFFECTS OF TURBINE POWER CONTROL VALVE ACTUATION 
NOZZLE AND HOT BLEED PORT (CON'T)

III ORIGINAL HOT BLEED PORT CONCEPT

D. NERVA HOT BLEED PORT REOUIREMENTS

1. HOT BLEED PORT REFINEMENTS

A. ENLARGE THE PORT DIAMETER TO $4.0 \mathrm{IN}$.

B. MAKE DILUENT FLOW INDEPENDENT OF NOZZLE COOLING SYSTEM

C. TO FACILITATE DEVELOPMENT TESTS PROVIDE A BOLT-ON JOINT BETWEEN THE PORT ASSEMBLY AND THE NOZZLE 


\section{NOZZLE AND HOT BLEED PORT (CON'T)}

IV DEVELOPMENT PLAN FOR NOZZLES

1. PROCURE FOUR NOZZLES TO SUPPORT DEVELOPMENT PROGRAM

2. SUPPLY ONE UNIT FOR ENGINE COLD FLOW SYSTEM DEVELOPMENT TESTS

3. DELIVER THREE UNITS FOR REACTOR TESTS NRX-A3, -A4, AND -A5

4. CONDUCT 18 NOZZLE DEVELOPMENT AND SIMULATION TESTS BY FEB 1965

5. ESTABLISH NOZZLE ULTIMATE COOLING CAPABILITY (FAILURE POINT) FEB 1965

6. CONDUCT 2 ACCEPTANCE TESTS ON EACH OF DELIVERABLE UNITS

7. CONDUCT 28 HOT BLEED PORT DEVELOPMENT TEST

8. CONDUCT 6 FINAL REFINEMENT VERIFICATION TEST JUNE 1965

9. CONDUCT 1200 SECONDS ENDURANCE TESTS ( 12 - 100 SEC) SEPT 1965 


$$
\text { NOZZLE AND HOT BLEED PORT (CON'T) }
$$

$\checkmark$ DEVELOPED TECHNOLOGY (FUTURE APPLICATIONS)

1. RELIABLE BRAZING TECHNINUE NOT RFSTRICTED BY THE NOZZLE SIZE

2. PROVEN FABRICABILITY AND PROCESS REPRODUCIBILITY SUITABLF FOR APPL ICATION TO LARGER UNITS

3. DEVELOPED EXTERNAL AND INTERNAL LEAK-FREE SYSTEM ELIMINATING POTENTIAL PROBLEMS ON FUTURE PROJECTS

4. DEVELOPED TURBINE DRIVE SYSTEM SUITABLE FOR APPLICATION TO FUTURE PROJECTS 
NOZZLE AND HOT BLEED PORT (CONT'D)

U-TUBE NOZZLE TEST DATA SUMMARY

\begin{tabular}{|c|c|c|c|c|}
\hline NOZZLE & $\begin{array}{l}\text { PC } \\
\text { CHAMBER } \\
\text { PRESSURE } \\
\text { PS IA }\end{array}$ & $\begin{array}{l}\text { FS } 1 \text { FS } \\
\text { DURATION } \\
\text { SEC }\end{array}$ & $\begin{array}{l}\quad \text { MR } \\
\text { MIXTURE } \\
\text { RATIOO }\end{array}$ & REMARKS \\
\hline$S / N 008$ & 471 & 1.6 & 6.15 & $\begin{array}{l}\text { AUTOMATIC SHUTDOWN BY FAULTY } \\
\text { COMBUST ION STABILITY MONITOR. } \\
\text { NO DAMAGE TO COMPONENTS }\end{array}$ \\
\hline$S / N 008$ & 625 & 6.9 & 9.3 & $\begin{array}{l}\text { TUBE EROS IONS CAUSED BY IN JECTOR } \\
\text { FAILURE. TERMINATED TEST SER IES }\end{array}$ \\
\hline$S / N 009$ & 499 & 6.1 & 3.9 & $\begin{array}{l}\text { INJECTOR EROSION. NO DAMAGE } \\
\text { TO NOZZLE. REPLACED INJECTOR }\end{array}$ \\
\hline$S / N 009$ & 555 & 4.6 & 8.3 & $\begin{array}{l}\text { TUBE DENTED BY EJECT ING IGN ITER } \\
\text { PART ICLES. REMOVED UNIT FROM } \\
\text { THE STAND FOR TUBE REPAIR }\end{array}$ \\
\hline$S / N 009$ & 660 & 5.8 & 4.1 & $\begin{array}{l}\text { ERODED IN IECTTOR MINOR TUBE } \\
\text { EROS IONS IN THROAT AREA. } \\
\text { TERMINATED TEST SERIES }\end{array}$ \\
\hline
\end{tabular}


NOZZLE FABRICATION

"U" TUBE - IST

FORGING ACQUISITION

PREGROOVE MACHINING GROOVING OPERATION

BRAZE AND LEAK TEST

FINAL MACHINE

FORGING ACQUISITION PREGROOVE MACHINING GROOVING OPERATION BRAZE AND LEAK TEST FINAL MACHINE
17

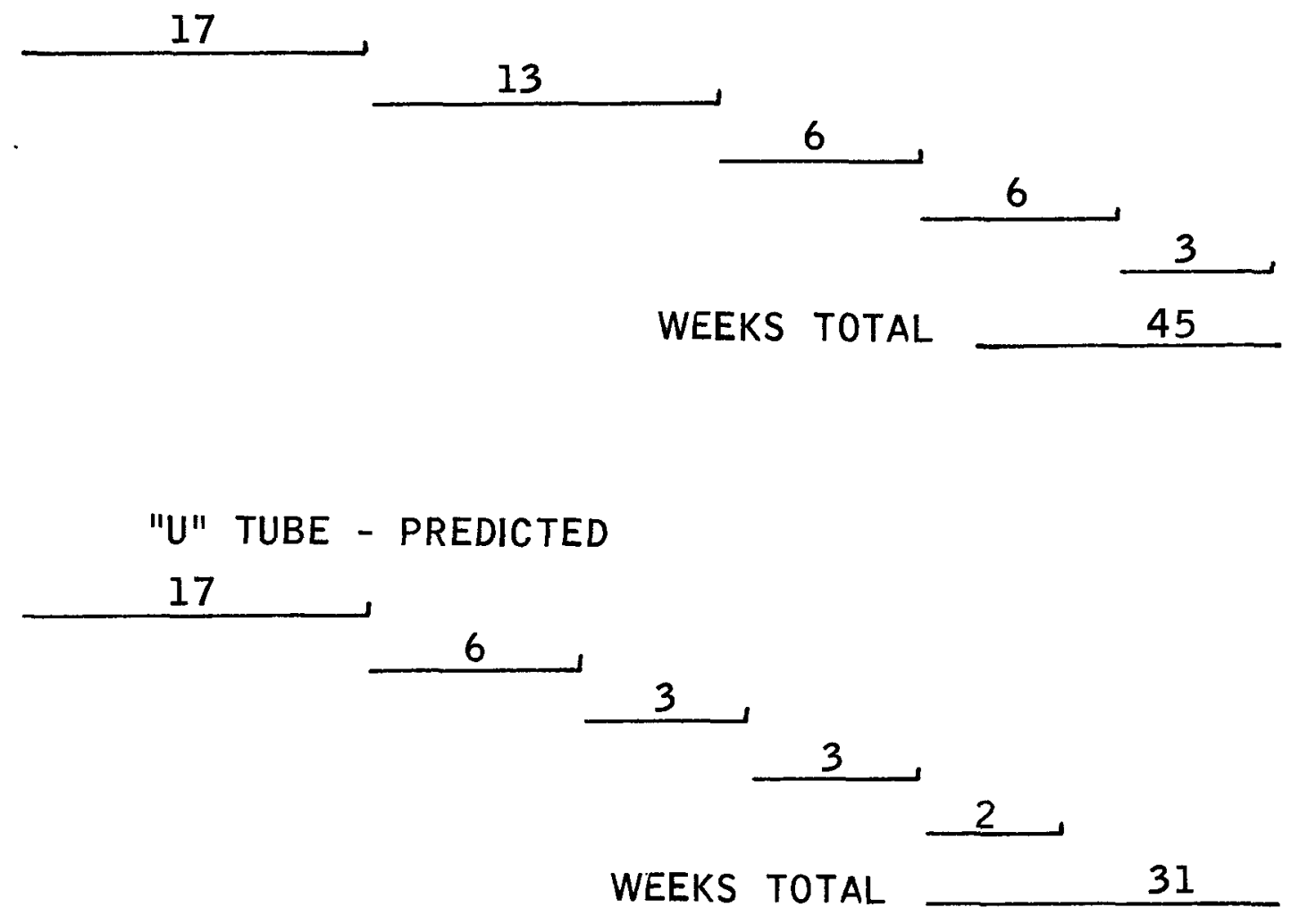




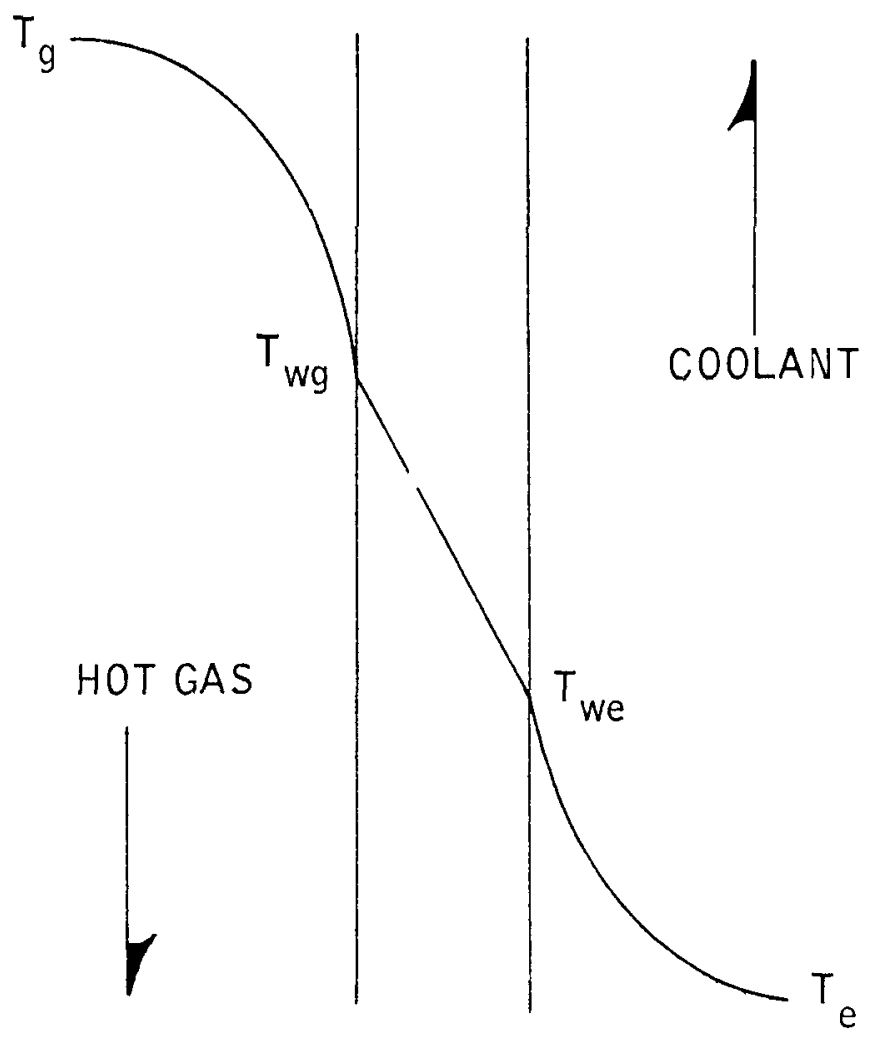

$$
Q^{\prime} A=U\left(T_{g}-T_{e}\right)
$$$$
\text { WHERE } \frac{1}{u}=\frac{1}{h_{g}}+\frac{x_{w}}{k_{w}}+\frac{1}{h_{e}}
$$

$\left.h_{g}\right)$ FROM "BEST ESTIMATE"

$h_{e}$ ) SCALE-MODEL CORRELATIONS

UNCERTAINTIES RESULT FROM

- SCALE FACTORS

- GEOMETRY VARIATIONS

- MODEL NON-SIMILARITY 
$\mathrm{NRX}-\mathrm{A} 2$

$1610^{\circ} \mathrm{F}$

1. FORWARD IMPINGEMENT $\mathrm{Cg} .067$

$1 / 3$ CON. SECT.

2. 1.2 Cg PRE NRX-A2

3. $1.05 \mathrm{He}$ (PRE NRX-A2)

4. COOLANT FLOW AREA

AT THROAT
$N R X-A 3$

AND SUBSEQUENT UNITS

$1410^{\circ} \mathrm{F}$

$1570^{\circ} \mathrm{F}$

$1370^{\circ} \mathrm{F}$

6.2 SQ. IN.

10.4 SQ. IN.

$\begin{array}{ll}1780^{\circ} \mathrm{F} & 1570^{\circ} \mathrm{F} \\ 1580^{\circ} \mathrm{F} & 1370^{\circ} \mathrm{F} \\ 10.4 \text { SQ. IN. } & 6.2 \mathrm{SQ} . \mathrm{IN} .\end{array}$




\section{HOT GAS SIDE WALL TEMPERATURE}

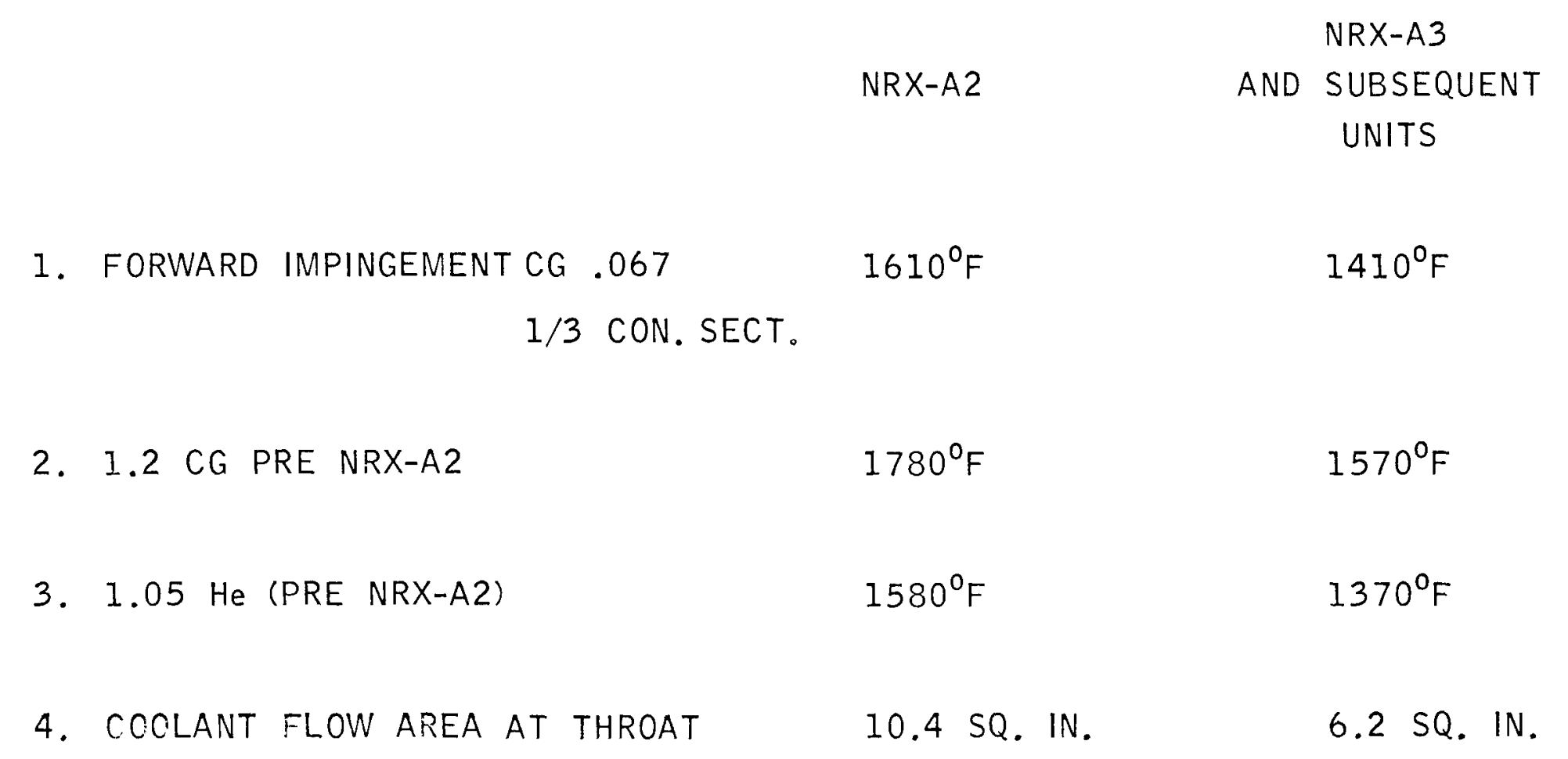


1. KAMT 51-1 USED WITH COPPER FACE PLATES IN INJECTOR S/N 260.

A. LIKE-ON-LIKE IMPINGING FUEL ORIFICES.

B. SHOWERHEAD OXIDIZER ORIFICES.

C. APPROXIMATELY CONSTANT M.R. ACROSS FACE OF INJECTOR.

D. FACE COOLANT TUBES IN FIFTEENTH CHANNEL.

E. PRESSURE DROP ACROSS OXIDIZER FACE PLATES TO BE GREATER THAN 120 PSI.

F. PRESSURE DROP ACROSS FUEL FACE PLATES TO BE APPROXIMATELY 30 40 PSI.

2. KAM 5I-8 USED WITH STAINLESS STEEL FACE PLATES IN INJECTOR S/N 260.

A. LIKE-ON-LIKE IMPINGING FUEL ORIFICES.

B. SHOWERHEAD OXIDIZER ORIFICES.

C. HIGH M.R. AT CENTER OF INJECTOR TAPERING TO LOW M.R. AT PERIPHERY.

D. PRESSURE DROP ACROSS OXIDIZER FACE PLATES TO BE GREATER THAN 120 PSI.

E. PRESSURE DROP ACROSS FUEL FACE PLATES TO BE APPROXIMATELY 3040 PSI. 
1. COPPER FACE PLATE INJECTORS:

A. PRESSURE DROP ACROSS OXIDIZER FACE PLATES WAS INCREASED TO REDUCE MAJOR INJECTOR EROSION.

B. ORIFICE PATTERN IN SIXTH CHANNEL WAS CHANGES TO MOVE ALL HOLES AT LEAST ONE INCH FROM BAFFLE TO ELIMINATE BAFFLE EROSION.

C. ADDED FACE COOLANT TUBES IN FIFTEENTH CHANNEL TO ELIMINATE EROSION OCCURING IN SIXTEENTH AND SEVENTEENTH CHANNELS.

2. STAINLESS STEEL FACE PLATE INJECTORS:

A. TO ELIMINATE EROSION OF TENTH, TWELFTH, FOURTEENTH, SIXTEENTH, AND SEVENTEENTH CHANNELS, THE MIXTURE RATIO ACROSS THE FACE OF THE INJECTOR WAS REALIGNED TO BE HIGH AT THE CENTER AND LOW AT THE PERIPHERY.

A TOTAL OF FIVE PATTERN CONFIGURATIONS WERE TESTED DURING 1964. 
EXTERNAL SHIELD

$\frac{\text { DESIGN }}{\text { DESIGN LAYOUT }}$
DESIGN CRITERIA
DETAIL DESIGN
FINAL ASSY. DESIGN
NUCLEAR ANALYSIS
STRUCTURE ANALYSIS
I.C.D. COORDINATION
COMPLETE DESIGN PACKAGE
DESIGN FREEZE
FABRICATION
INITIATE FABRICATION
DELIVERY
INSPECTION AND TESTING

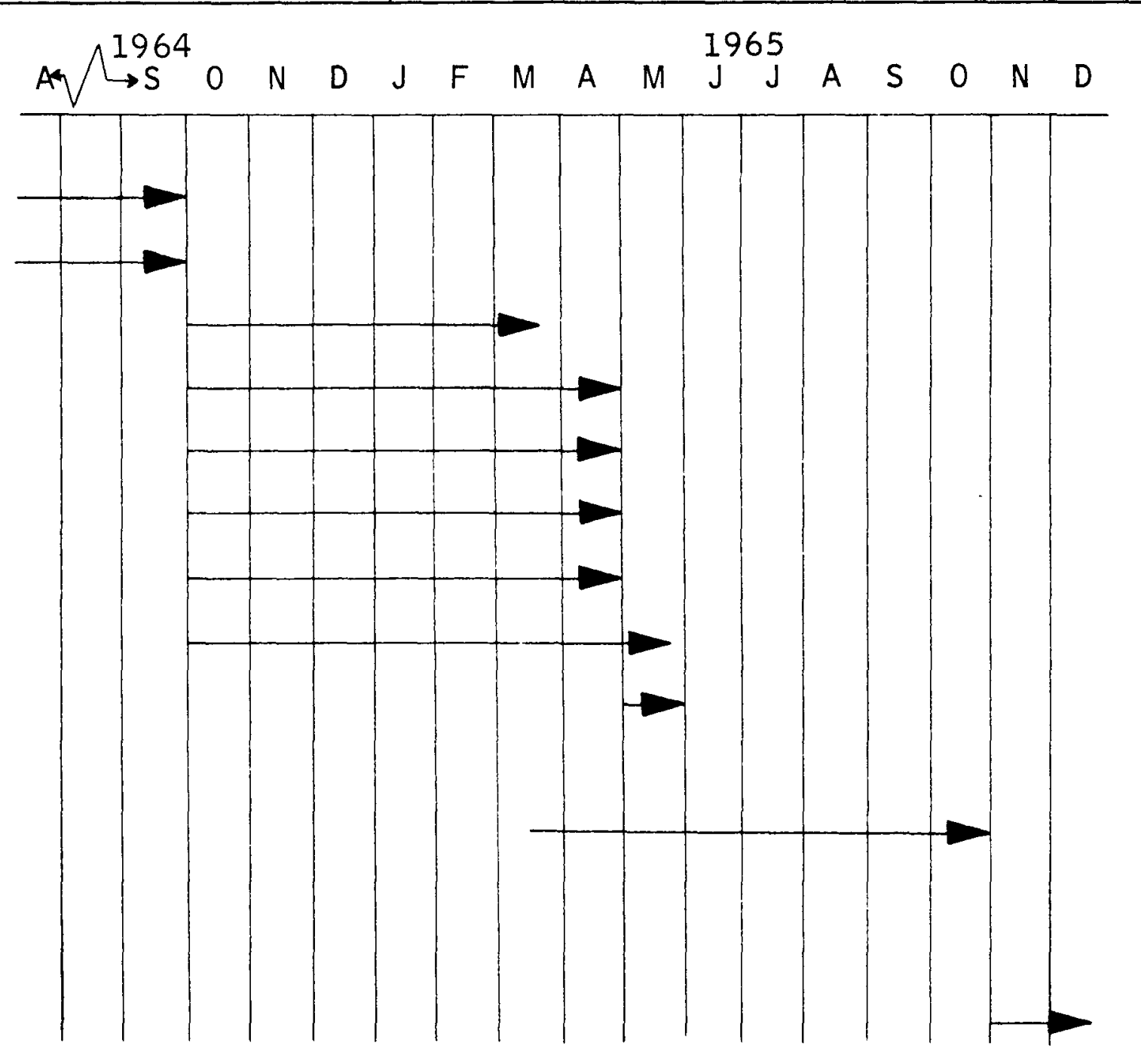


1. DESIGN AND DOCUMENTATION

A. MASTER LAYOUT - 95\% COMPLETE

B. DETAIL DRAWINGS - IN DESIGN PHASE, $10 \%$ COMPLETE

C. DETAIL SPECIFICATION - ISSUED 7 NOV. 1964

D. INTERFACE DRAWING LAYOUT - IN PROCESS, 50\% COMPLETE

2. TOOLING

A. MATCH PLATE GAGES - IN DESIGN, 10\% COMPLETE

3. FABRICATION

A. MOCKUP - IN FABRICATION

4. PROCUREMENT

A. ADVANCE COST QUOTES - RECEIVED ON PRIMARY COMPONENTS

B. BALL BEARINGS - REQUISITION PREPARED 
MATCH PLATES

\section{DESIGN}

DETAIL DESIGN

PRELIMINARY REVIEW

FINAL REVIEW

\section{FABRICATION}

\section{FABRICATION}

\section{DELIVERY}

INSPECTION \& ACCEPTANCE

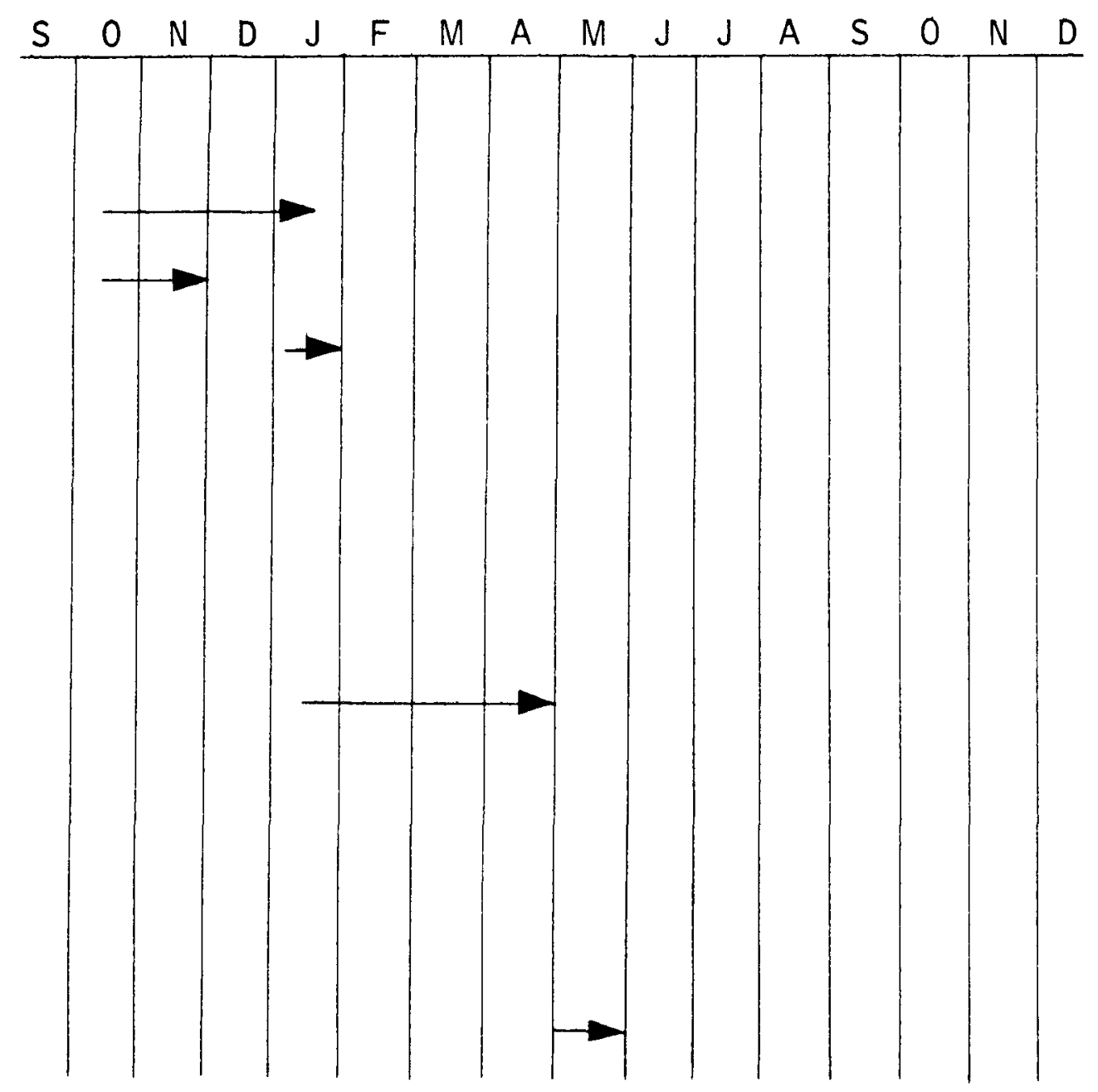


1. DEVELOPED SATISFACTORY MATERIAL PROPERTIES IN CAST ALUMINUM JACKETS

2. PROVIDED TWO UNITS (NRX-A1 AND NRX-A2) TO NRDS

3. DEVELOPED SATISFACTORY BRAZE JOINT BETWEEN THE STEEL NOZZLE SHELL AND THE COOLANT CHANNELS

4. DEMONSTRATED TEST SUPPORT EQUIPMENT CAPABILITY OF EXTENDED TEST DURATION 


\section{NOZZLE COOLANT TUBE}

\section{FLOW, SOLID, AND SURFACE NODE REPRESENTATION}

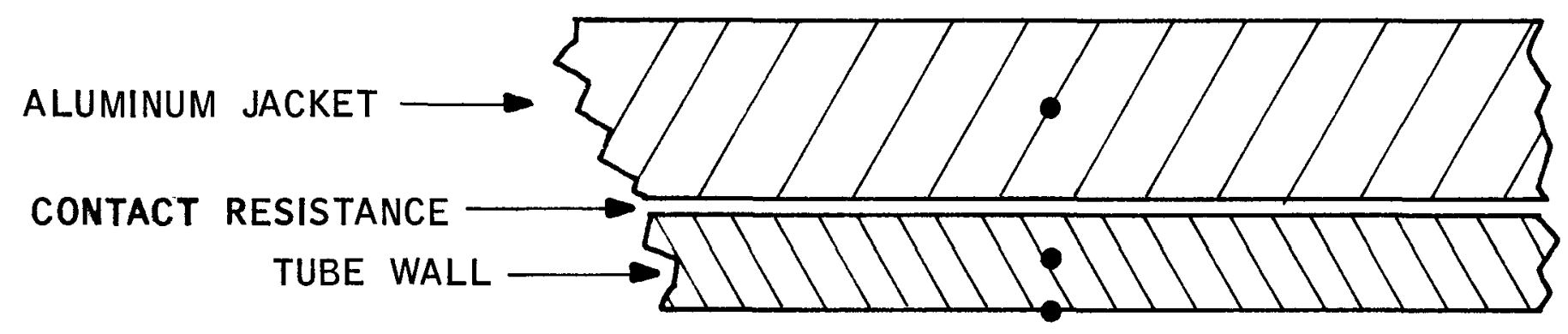

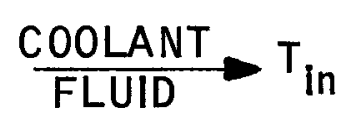
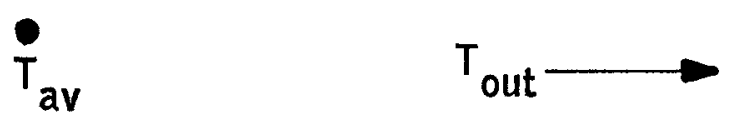

TUBE WALL

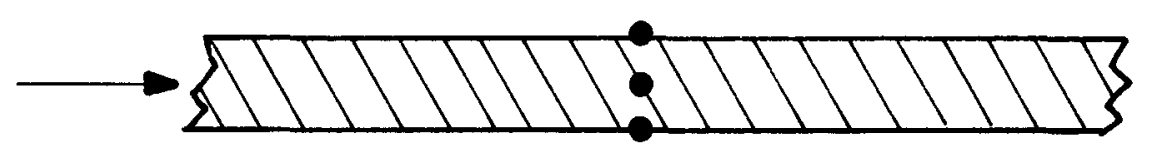

$+\frac{\text { HOT }}{\text { GAS }}$ 


\section{MECHANICAL PROPERTIES}

\begin{tabular}{|c|c|c|c|c|}
\hline NOZZLE & DATE CAST & UTS (RSI) & YS (KSI) & ELONG $(\%)$ \\
\hline \multirow{2}{*}{$001 \mathrm{H}$} & $11 / 28 / 62$ & 27.5 & 26.9 & 1.0 \\
\hline & & 28.5 & 28.5 & 0.8 \\
\hline \multirow[t]{2}{*}{$002 \mathrm{~L}$} & $5 / 01 / 63$ & 28.6 & 23.5 & 2.5 \\
\hline & & 28.6 & 25.4 & 1.5 \\
\hline \multirow[t]{2}{*}{0004} & $5 / 29 / 63$ & 34.2 & 27.6 & 3.0 \\
\hline & & 33.2 & 28.7 & 3.0 \\
\hline \multirow[t]{2}{*}{0015} & $4 / 01 / 64$ & 40.3 & 25.5 & 15.0 \\
\hline & & 40.1 & 25.0 & 13.4 \\
\hline 0014 & $8 / 04 / 64$ & 35.7 & 23.8 & 15.8 \\
\hline & & POROSITY & & \\
\hline
\end{tabular}

001H NO LARGE ISOLATED VOIDS WERE PRESENT, HOWEVER THE JACKET CONTAINED SOME SMALL GAS VOIDS AND THE USUAL ROUND GAS POROSITY IN THE BACKGROUND.

002L ROUND GAS POROSITY WAS STILL PRONOUNCED THROUGHOUT THE JACKET.

0004 THIS JACKET WAS VIRTUALLY FREE OF ROUND GAS POROSITY, HOWEVER, IN ITS PLACE, FIND SHRINKAGE WAS DISPERSED THROUGHOUT THE FLANGE AREÁ.

0015 A SLIGHT AMOUNT OF SHADING WAS DISCERNIBLE, HOWEVER, THERE WERE NO CONCENTRATIONS OF POROSITY.

0014 THIS NOZZLE WAS COMPLIMENTARY TO 0015 EXCEPT THAT SMALL BLOWS WERE NOTED NEAR TWO OF THE THERMOCOUPLE PASS-THROUGHS. 



\section{-}

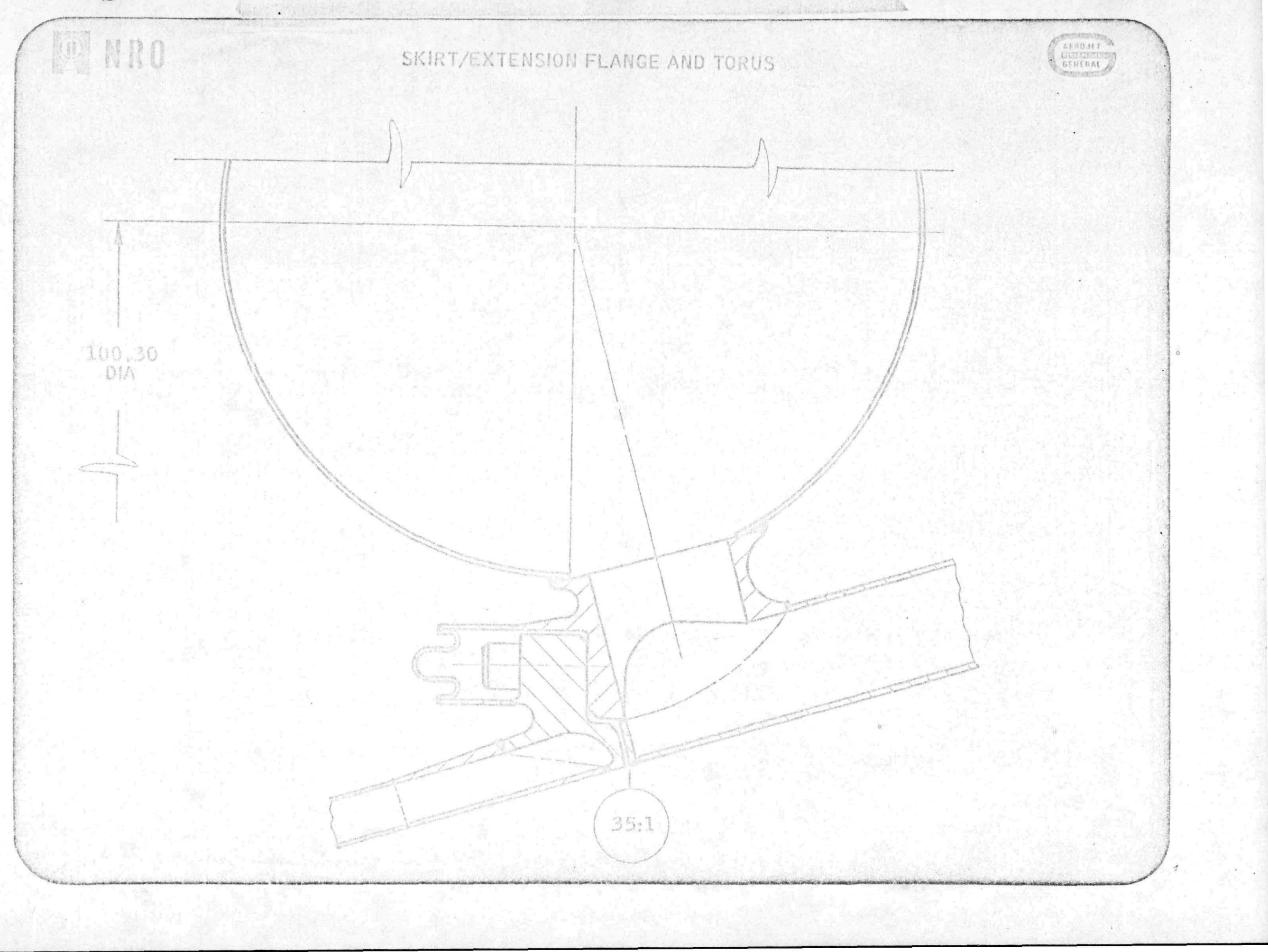





\section{NOZZLE-SKIRT COMPARISON}
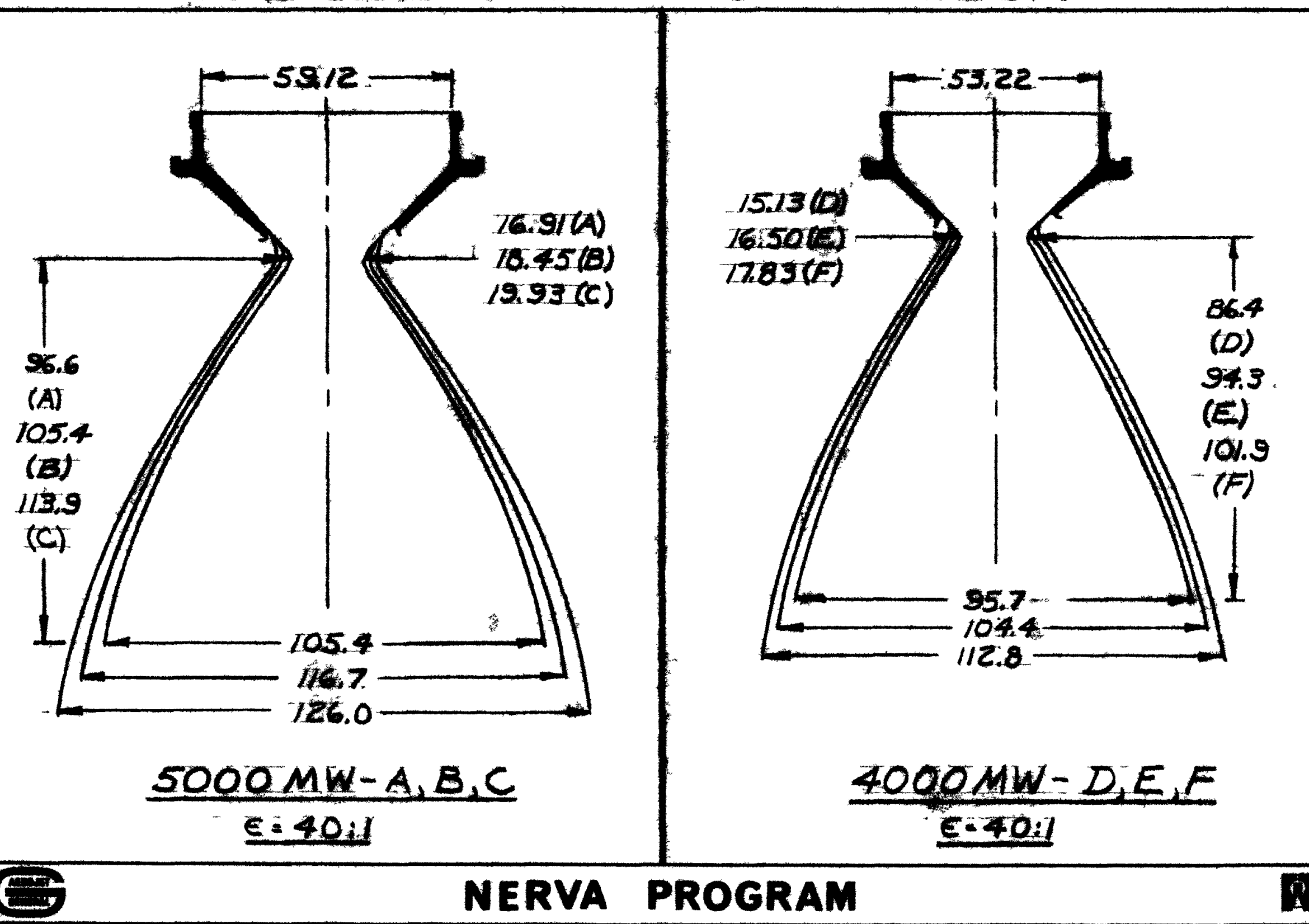

$5000 M W-A, B, C$ $E=40 . I$

$867-022$ 


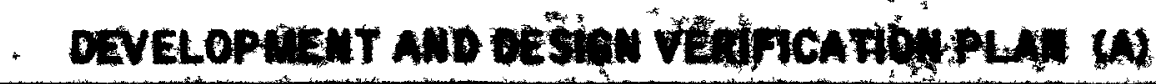

A. BLED PORT WISERT ASSEMBGAS

1. EVALOATE OPERATIMG CMARACTERISTICS OF FOUR DIFFEnIT MODELS BY SHMLATME THERMALLOADS

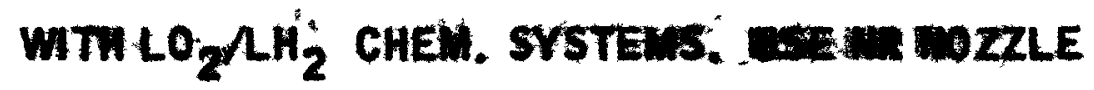
(TEST SULAMUMECY 2 MODELS PER TEST SERIES)

2. SELECT A MODEL SUITABLE FOR THE ENOME SIX MONTHS PRIOR TO DELIVERY OF THE FIRST UNIT

3. ESTABLISHETHE UL TIMATE OPERATIMG CAPABILITY OF THE SELECTED MODEL $\mathrm{MLO} \mathrm{L}_{2} \mathrm{LH}_{2}$ ATMOSPHERE BY:

a. GRADUAL REDUCTION OF COOLAIT FLOW RATE UNTIL FAILURE AT DESIGN HOT BLEED FLOW

b. GRADUAL INCREASE OF HOT BLEED FLOW UNITL FAILURE AT DESIGN COOLANT FLOW. VERIFY PREDICTION 
B. NOZZLE

1. INSTALL OPERATIONAL BLEED PORT INSERT IN ME MOZZLE

2. DEMONSTRATE NOZZLE OPERATIONAL CAPABILITY AT

DESIGN THERMAL AND PRESSURE INDUCED LOADS

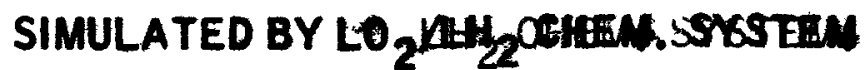

VERIFY CALCULATED $\triangle P, \triangle T$ AND WALL TEMPERATURES

3. ESTABLISH THE ULTIMATE OPERATING CAPABILITY USING $\mathrm{LO}_{2} \mathrm{RH}_{2}$ CHEM. SYSTEM BY:

a. GRADUAL INCREASING $P_{C}$ AT CONSTANT MIXTURE RATIO AND DESIGN NOZZLE COOLANT FLOW RATE WITHOUT EXCEEDING STRUCTURAL LIMITATIONS OF THE TEST ASSY

b. GRADUAL DECREASE OF COOLANT FLOW RATE AT CONSTANT MIXTURE RATIO AND MAXIMUM ATTAINED CHAMBER PRESSURE UNTIL FAILURE. VERIFY PREDICTION

4. PHOEBUGII PROGRAM 


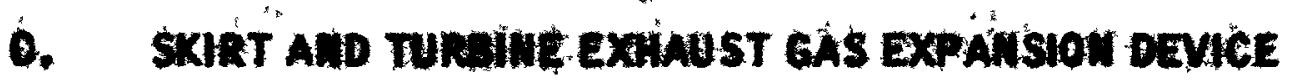

1. DEIOHSTRATE AMALTICALIY SATISFACTORY FUNCTIONAL

CHARAGTERISTICS AHD ADEQUATE THERMAL AND

STRUCTURAL MARGINS OF SAFETY

2. PERFORM APPROPRIATE HYDROSTATIC AND HYDRO-FLOW

TEST TO DETERMINE THE FOLLOWMG:

a. FLOW COEFFICIENTS

b. STRUCTURAL INTEGRITY

c. LEAK FREE ASSEMBLY

3. VERIFY FLOW CHARACTERISTICS (ME-CF)

4. VERIFY OPERATIONAL CHARACTERISTICS (NE-2)

5. IMCORPORATE ALL REFINEMENTS ON THE BASIS OF

FEEDBACK FROM RADA, NE-CF AND NE-2 INTO NE-4

AND FREEZE DESIGN 
D. LINES ASSEMBLIES AMD SUBCOMPOMENTS

I. SUBCOMPONENTS

a. GIMBAEDBELLOWS AMD RLEXIBLE SECTIONS

1. AMBIENT TEMPERATURE TESTING

LEAK AND PROOF TESTS,

ANGULATION CYCLING TEST,

PROOF, RE-LEAK AND BURST $\left(\mathrm{H}_{2} \mathrm{O}\right)$

2. CRYOGEMIC TEMPERATURE $\left(\mathrm{LN}_{2}\right)$

AMGULATION CYCLING AT PUMP DISCHARGE PRESS.,

PROOF, LEAK AND BURST $\left(\mathrm{H}_{2} \mathrm{O}\right)$

3. ELEVATED TEMPERATURE

ANEULATION CYCLING AT TUREINE IMLET OR

TURBINE DISCHAREE TEMP. AND PRESSURE,

PROOF, LEAK AND BURST Gy "

4. SELECT DESIEN FOR ME LIIES ASSY

NERVA PROGRAM 



\section{DEVELOPMENT AND DESREN VERIFICATION PLAI (D) - CONTO}

D. LINE ASSEMBLIES AND SUBCOMPONENTS

II. LINE ASSEMBLIES (WITH SELECTED SUBCOMPONENT CONFIGURATIONS)

a. LABORATORY TESTS

1. LEAK, PROOF AND VIBRATION TESTS

2. RE-LEAK, PROOF TEST

3. RELEAK AND BURST

b. OPERATIONAL TESTS

1. PUMP DISCHARGE LINE ASSY DES. VERIF. TESTS IN CONUUMCTION WITH TPA DEVELOPMENT $\left(\mathrm{H}_{2}\right)$

2. TURBINE DISCHARGE LINE ASSY DES. VERIF TESTS IN COWUNCTION WITH TPA DEVELOPMENT $\left(\mathrm{H}_{2}\right)$

3. TURBINE INLET LINE ASSY DES. VERIF TEST In CONJUACTION WIT BLEED PORT INSERTS AND NOZZLE DEVELOPMENT $\left(\mathrm{LO}_{2} / \mathrm{LH}_{2}\right)$ 
INTRODUCTION OF THE WODEL

1. PHYSICAL DESCRIPTION

2. NOZZLE CONTOUR JUSTIFICATION

3. NOZZLE AREA RATIO JUSTIFICATION

4. METHOD OF CONSTRUCTION

A. NOZZLE AND BLEED PORT INSERT ASSY

B. SKIRT

C. TURBINE EXHAUST EXPANSION DEVICE

5. COOLING FEASIBILITY STUDY

6. SELECTION OF THE COOLING SYSTEM

7. PARAMETRIC OPERATIONAL CHARACTERISTIC

8. DEVELOPMENT AND DESIGN VERIFICATION 


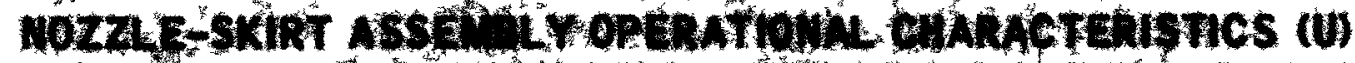

A. Mnnopres 270

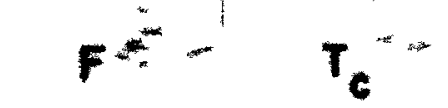

LAfo

on

$-1$

240,4500

$200 \quad 4500$

$150 \quad 4500$

$240 \quad 5000$

B. SKIRT

\begin{tabular}{r|rr|r}
240 & 4500 & 625 & 1244 \\
200 & 4500 & 517 & 1060 \\
150 & 4500 & 375 & 714 \\
240 & 5000 & 517 & 1060
\end{tabular}

$82 \quad 37,520$

360 .

117

690

$61 \quad 31,840$

270

$37 \quad 23,960$

190

135

720

$56 \quad 36,310$

260

160,850

160,920

MOTE: IN ALL CASES THE MOZZLE-SKIRT DESIEN AND THE AMOUNT OF SPECIAL PURPOSE COOLING WERE KEPT CONSTANT

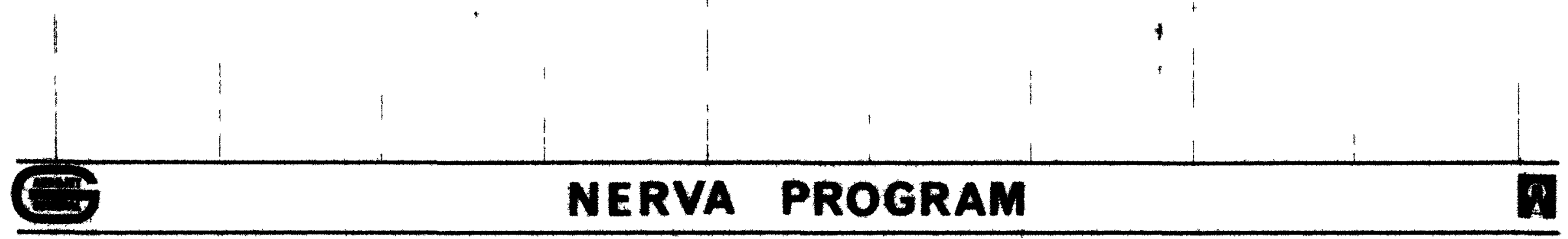




\section{NERVA NOZZLE DEVTLOPLENT}

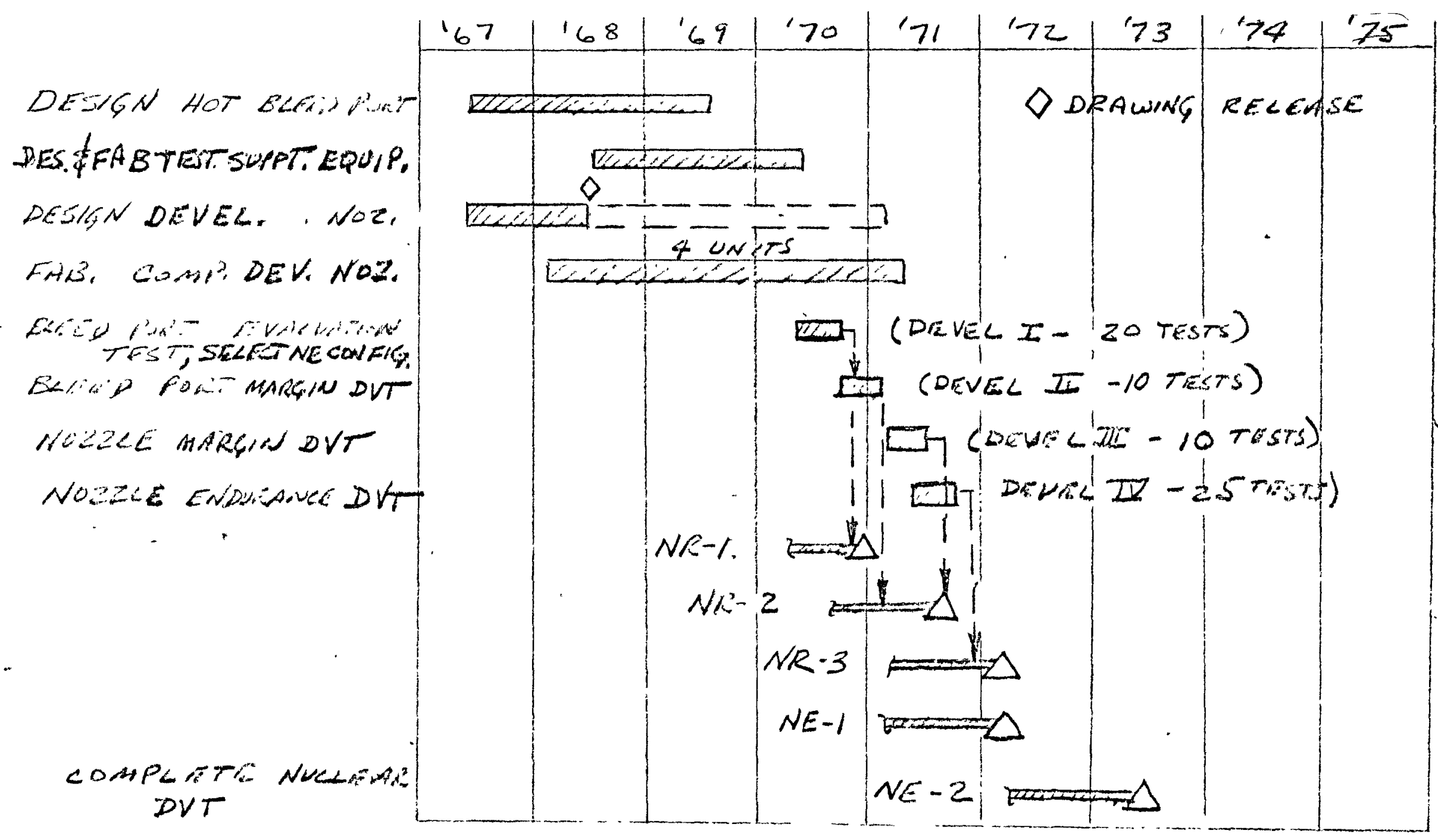


18) Nra

LINE JOINTS
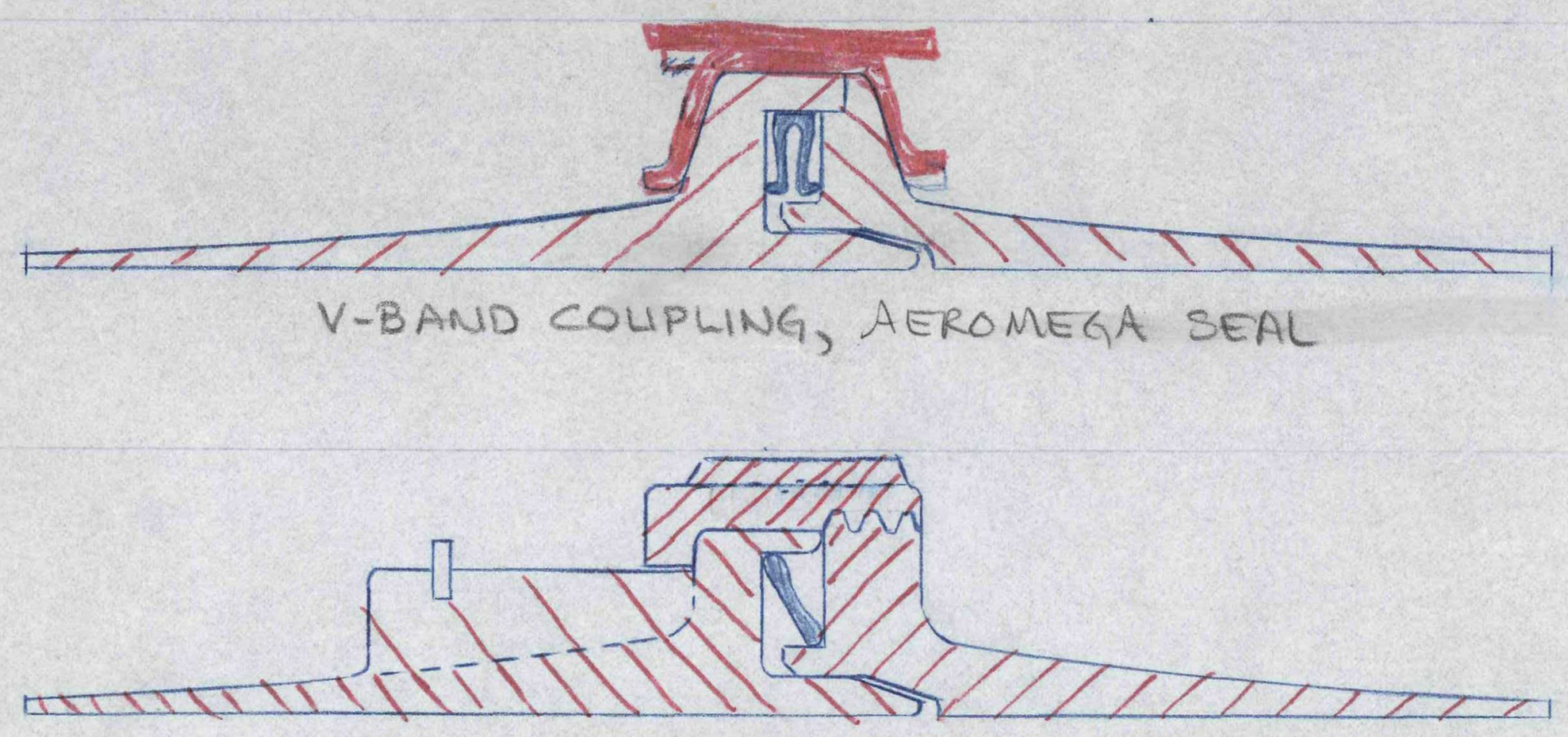

thrEADED COUPLING, INFUNDIBULAR SEAL

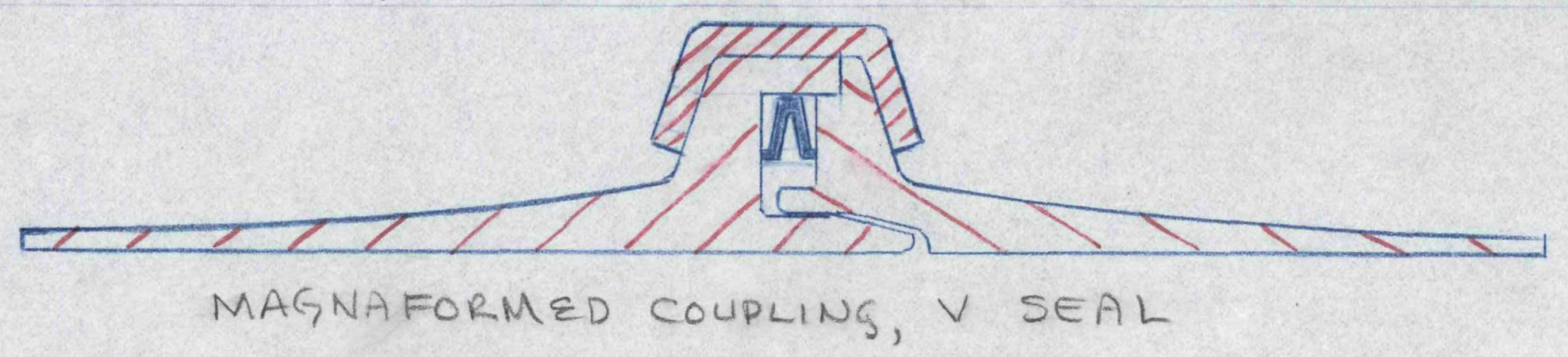




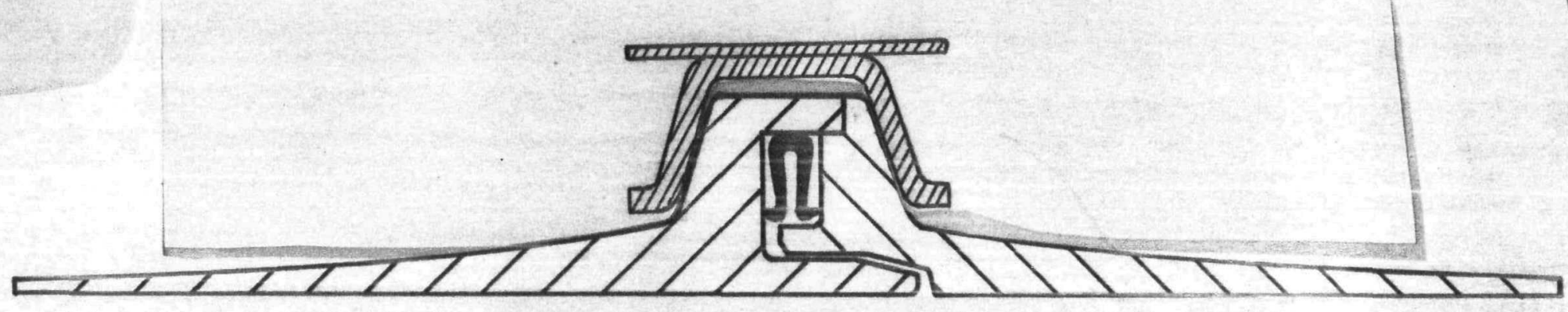

V-BAND COUPLING, AEROMEGA SEAL

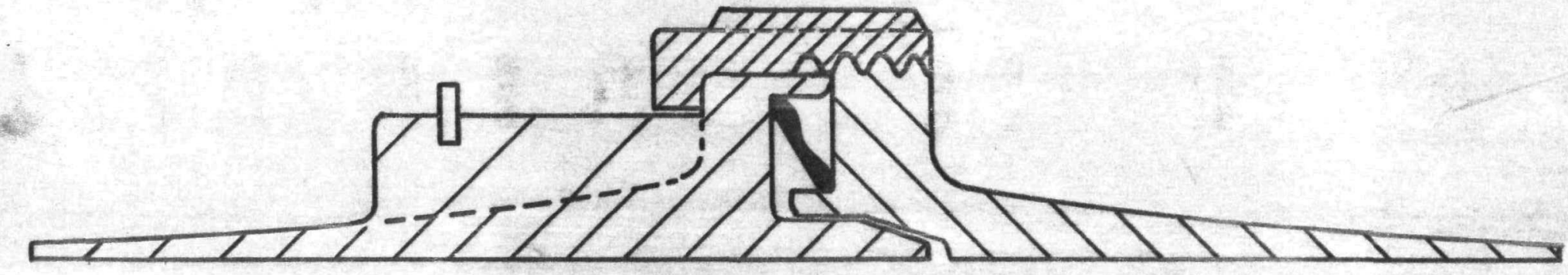

THREADED COUPLING, INFUNDIBULAR SEAL

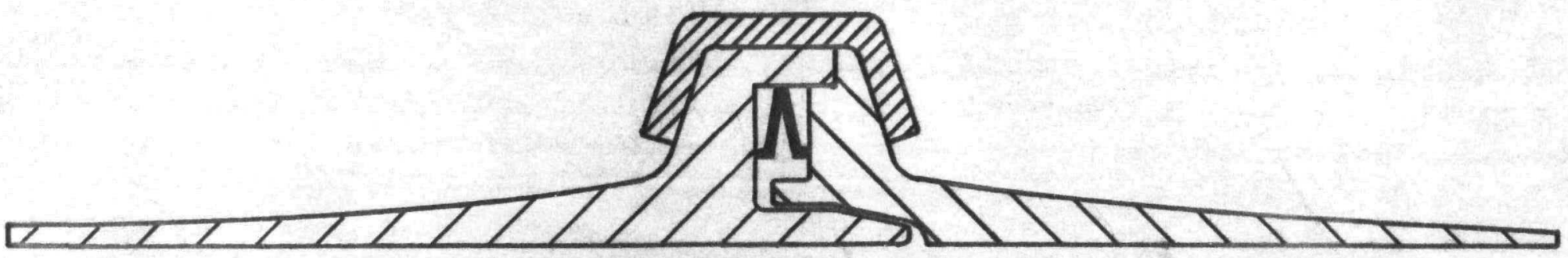

MAGNAFORMED COUPLING, V SEAL 
NOZZLE SKIRT PERFORMANCE FOR EQUAL. LENGTH NOZZLES

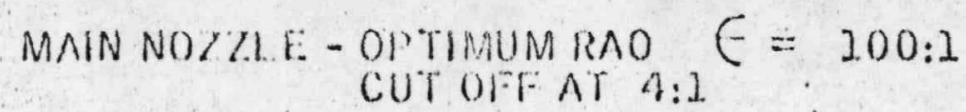

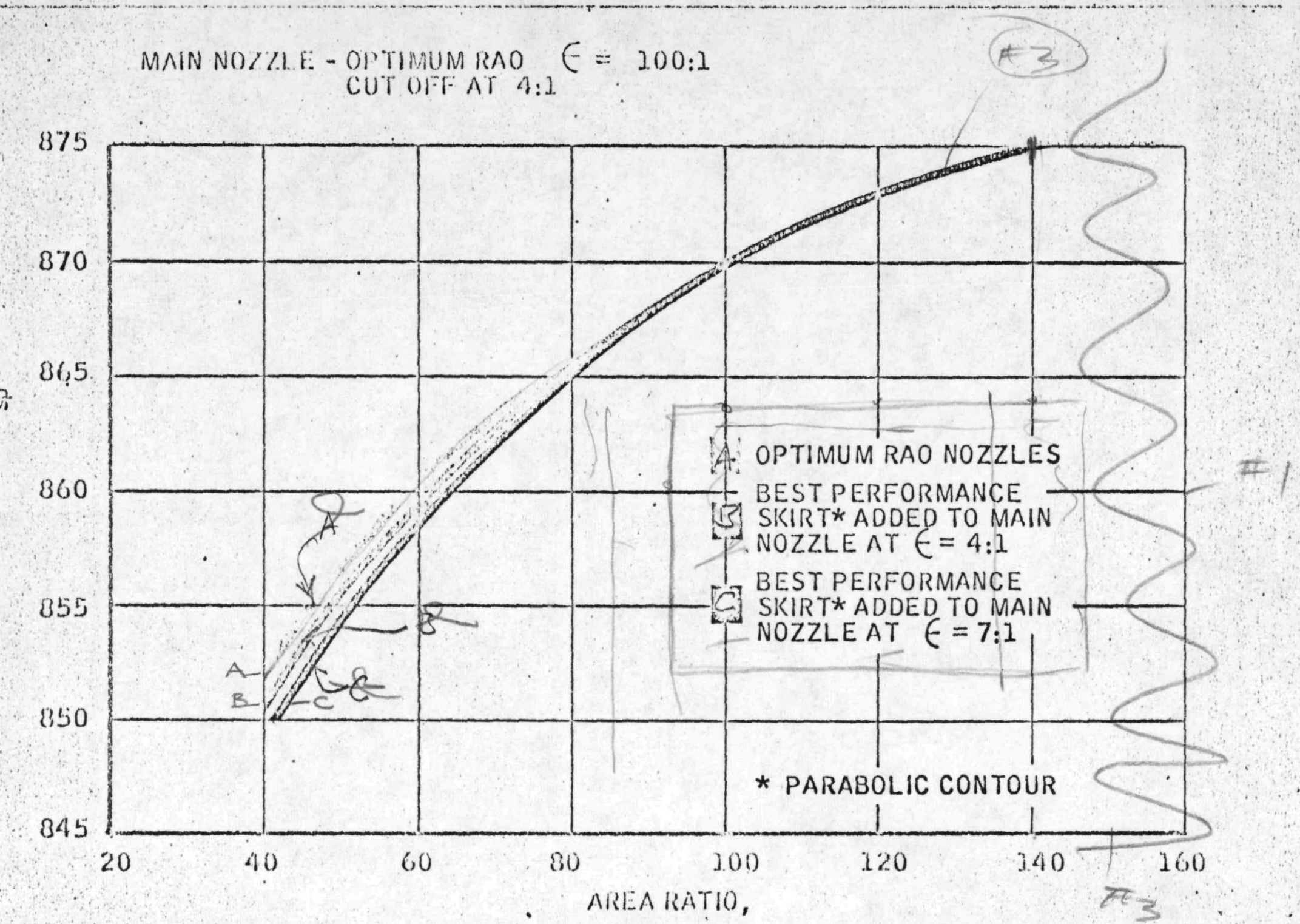


WEIATT: 132 LEB NETZ, LB NET)

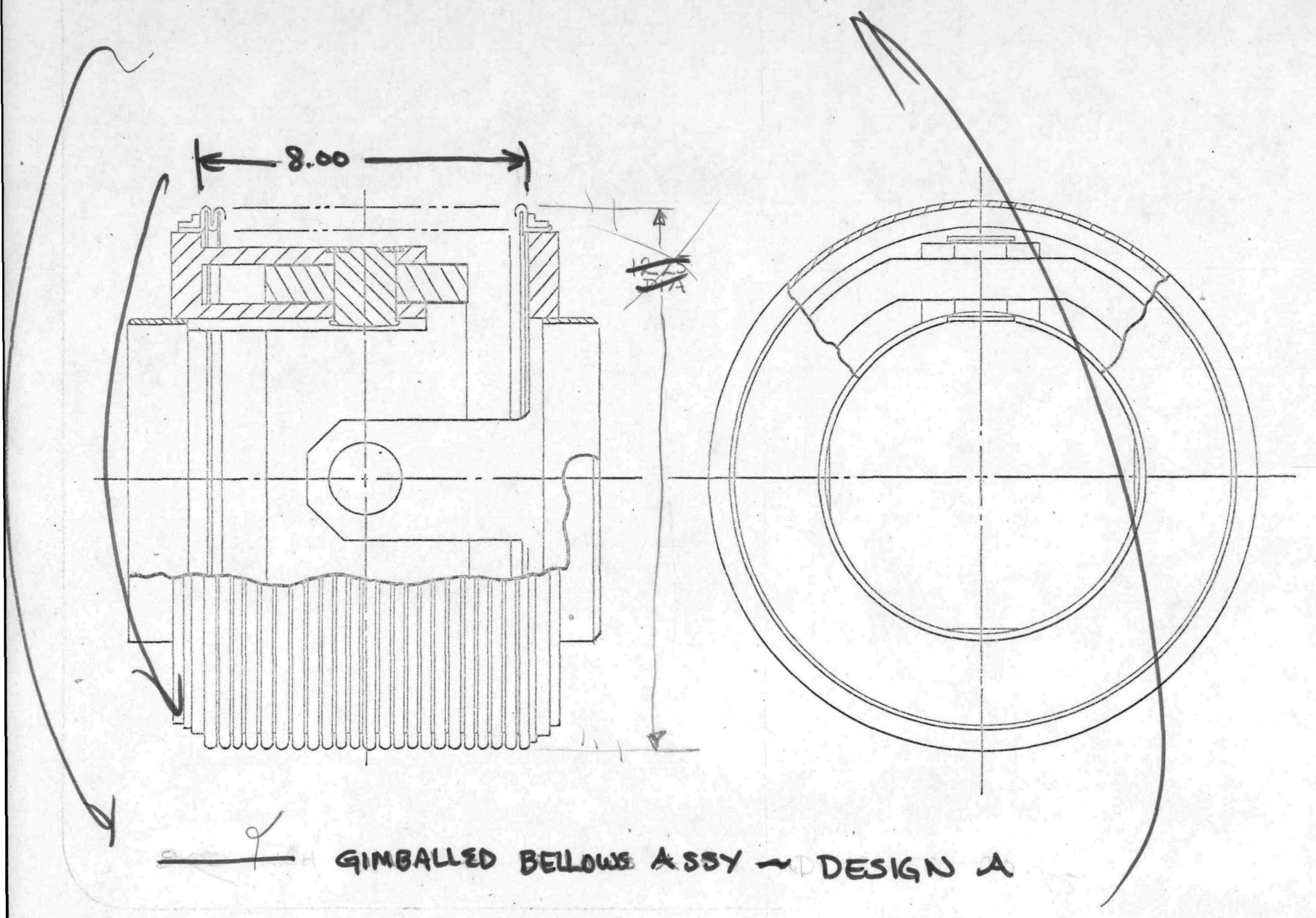


WEIGHT: $56, L B N E T$

$1888 \mathrm{~W}-\mathrm{LB} / \mathrm{DEG}$

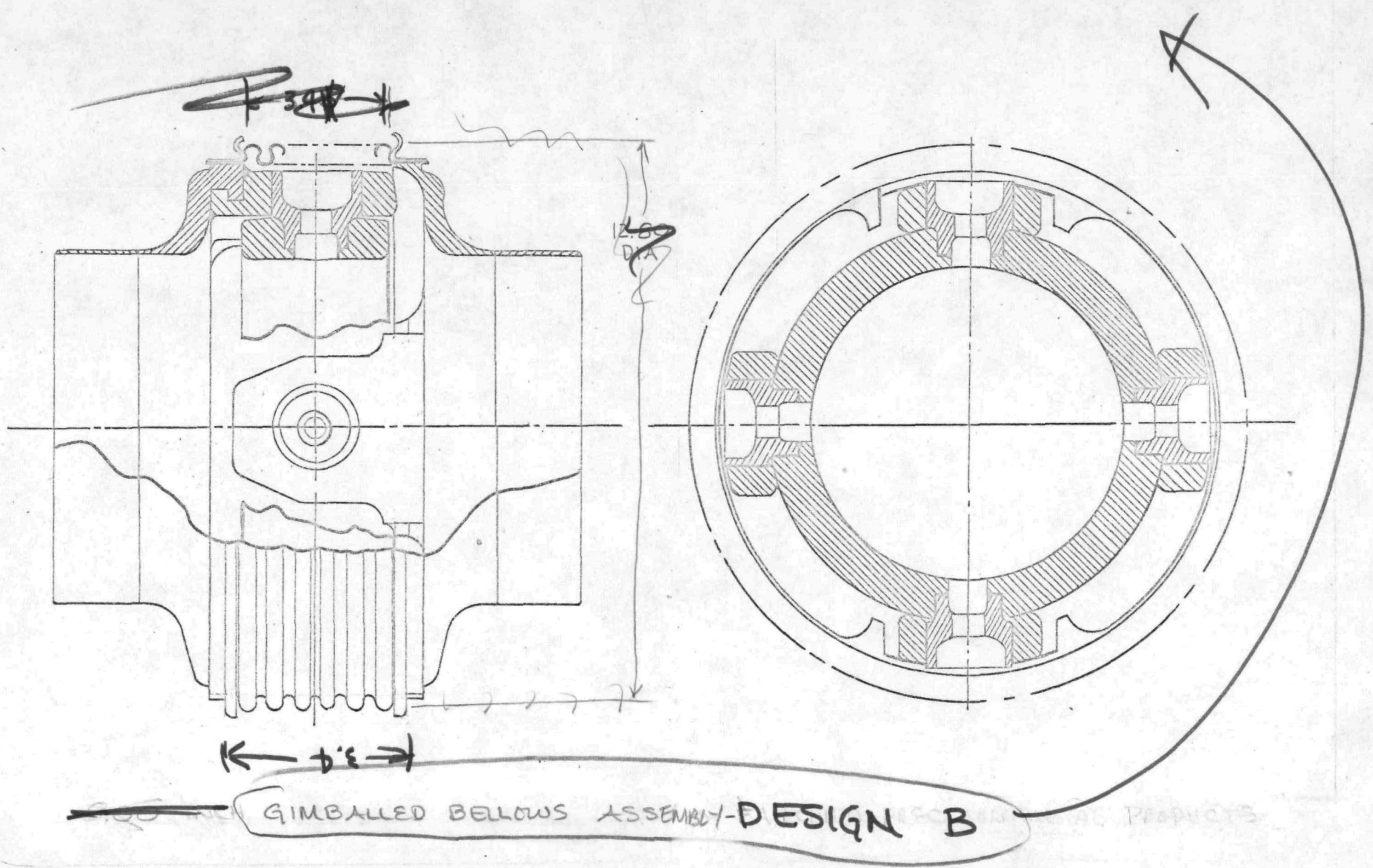

$867-074$ 
WEIGHT: 64:LB NET
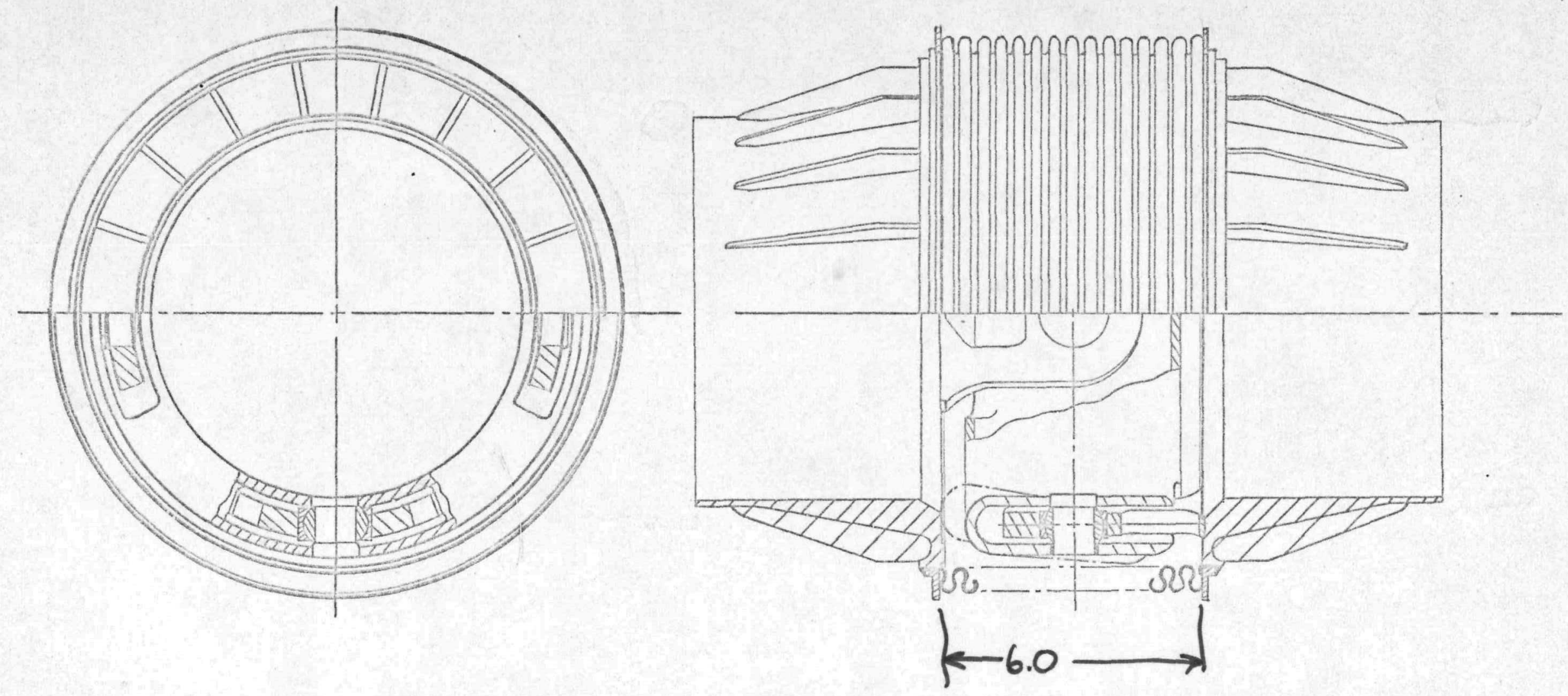

GIMBALlED BELLOWJ ASSENGLY $A G C$ DESIGN D 
WEIGHT: 38 NIF LB NET
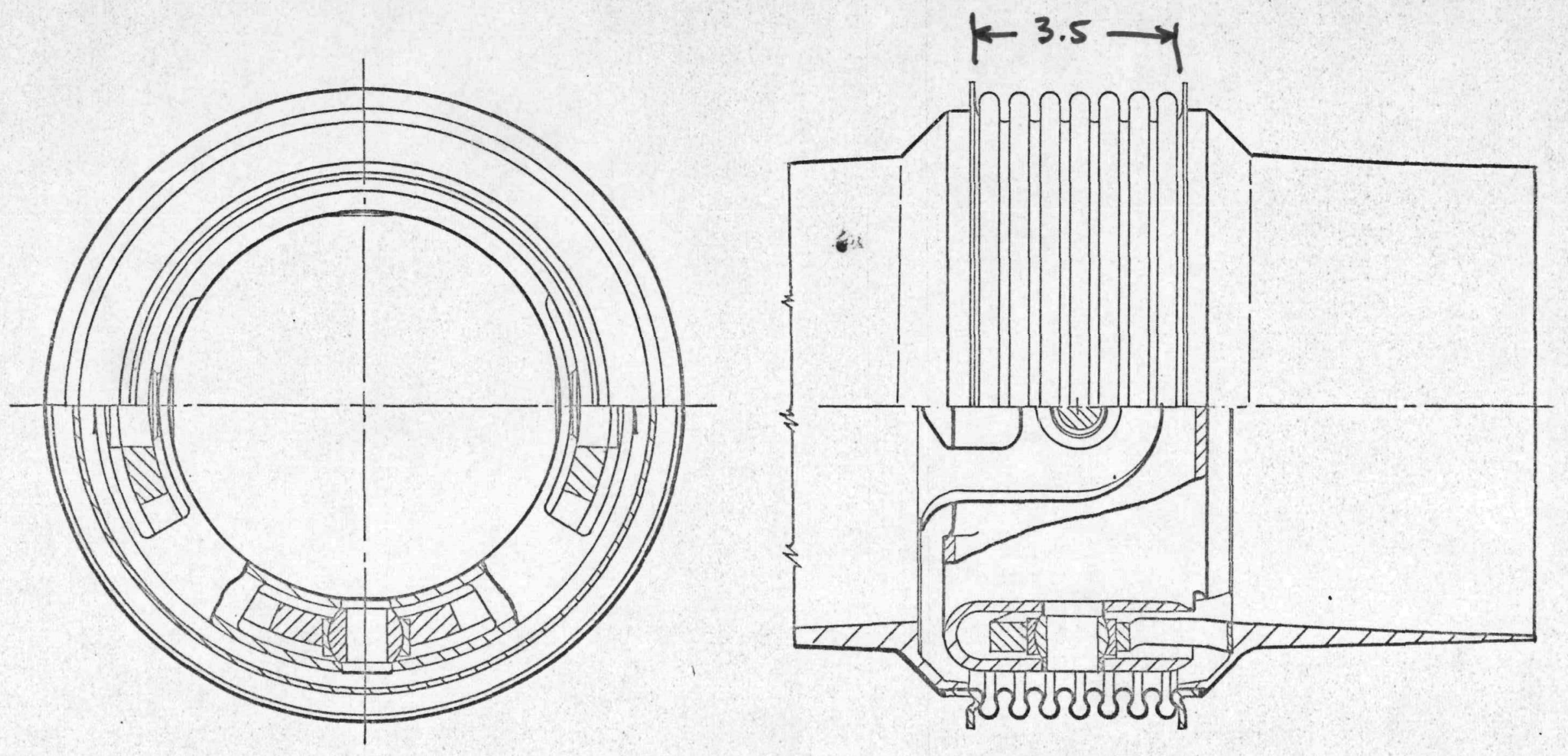

GIMBALLED BELLOWS ASSEMBLY AGC DESIGN $=E$ 


\section{-}

[9 NRO

SCREW CLAMP ( U SEFUL ONLY ABUVE SHELDED POME)
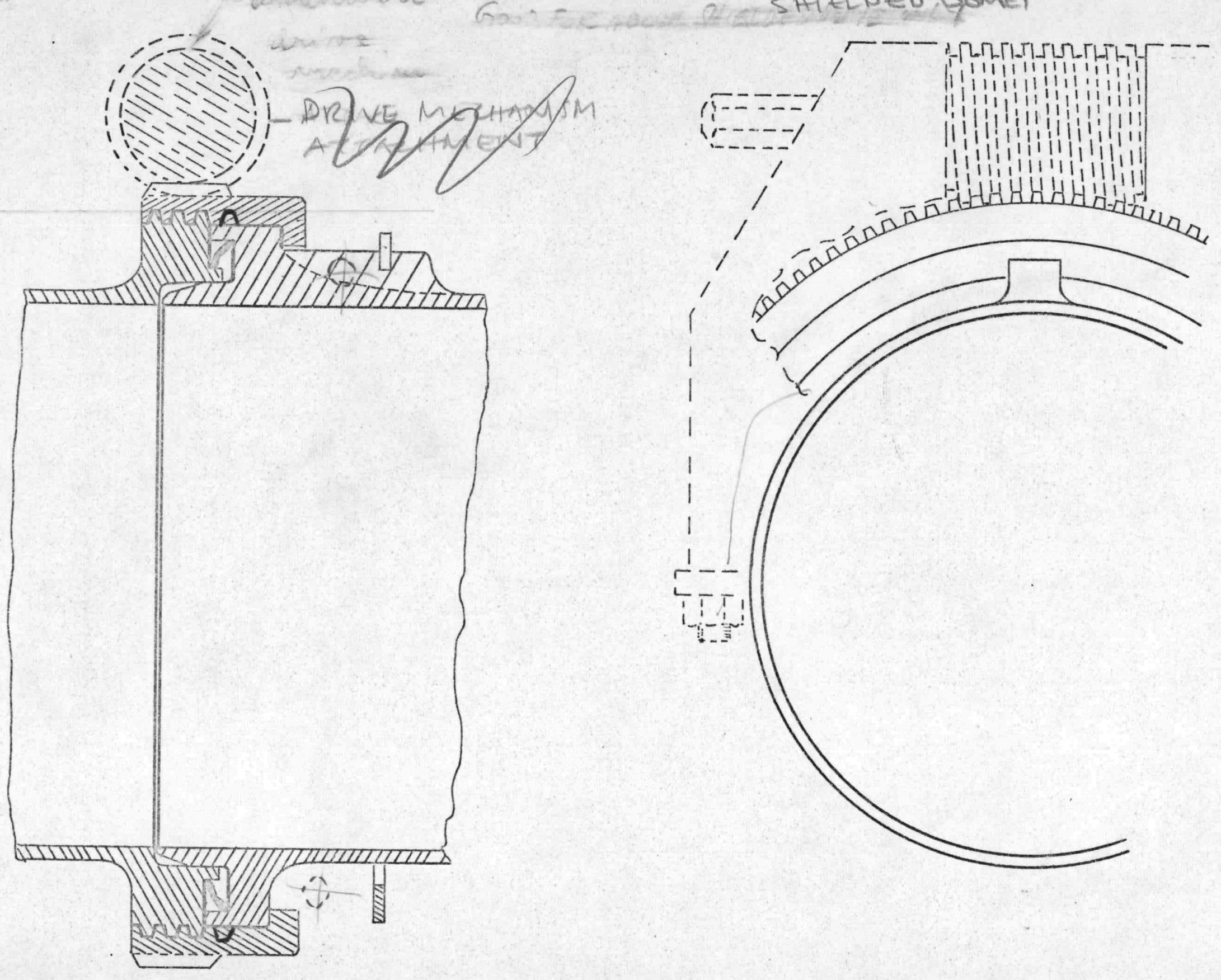
WNRO

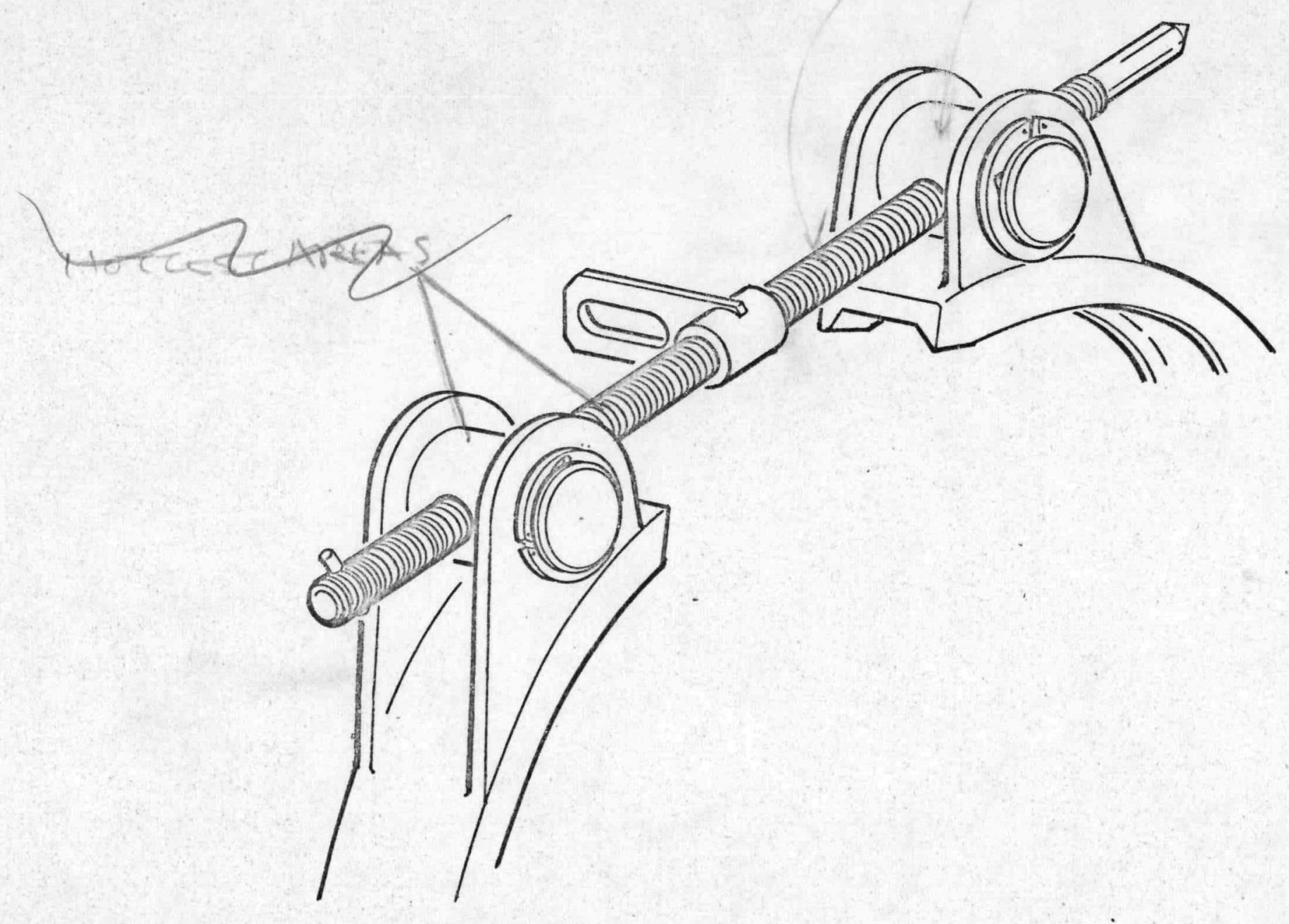


?

(W) NRO

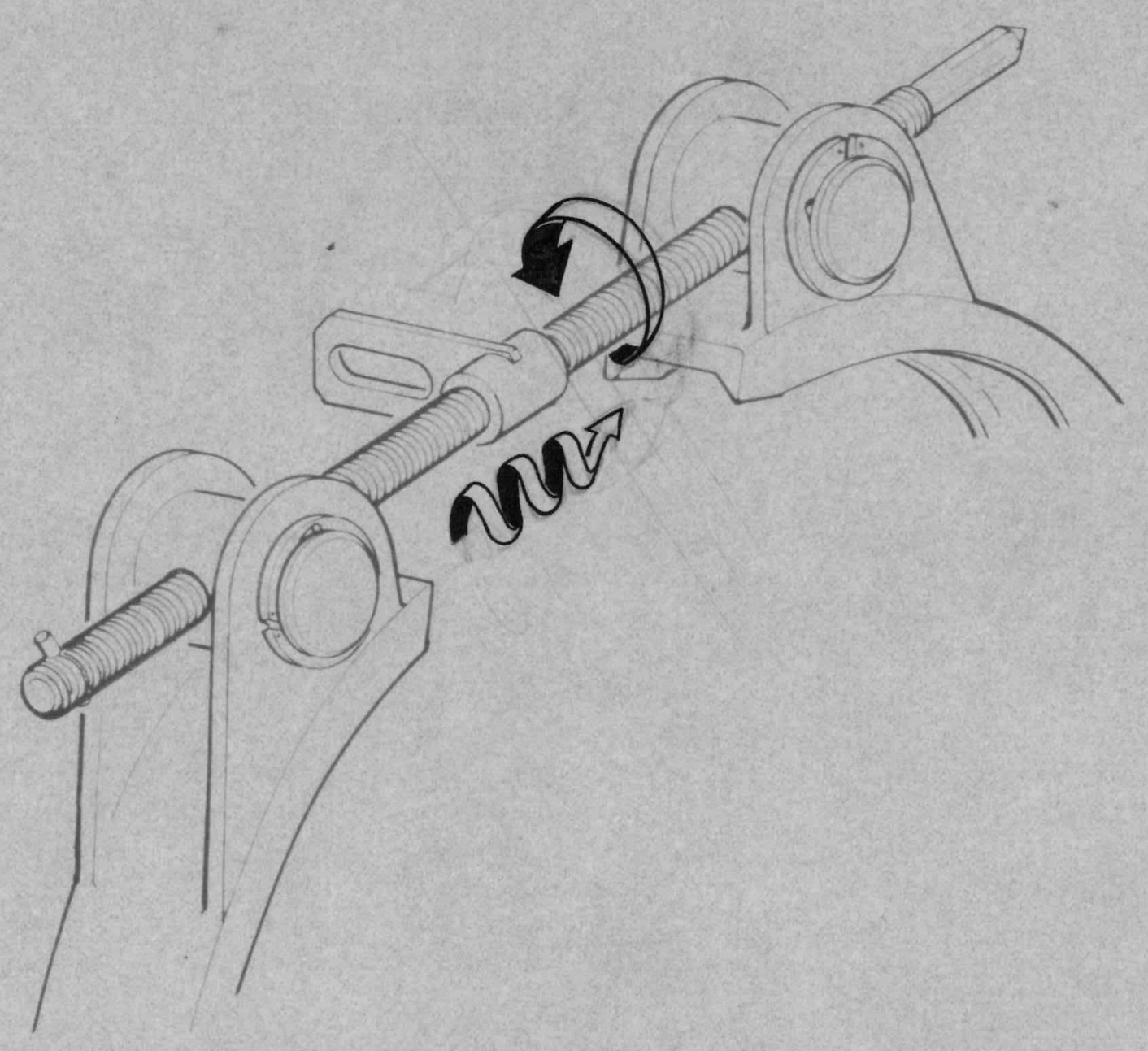


* 1. USE EXISTING GAS-SIDE CONTOUR MODIFY COOLANT PASSAGES TO MAINTAIN ACCEPTABLE WALL TEMPERATURES WITH AVAILABLE COOLANT FLOW.

*2A. INCREASE THE THROAT AREA TO REDUCE THE P ${ }_{C}$ AND ASSOCIATED GAS-SIDE HEAT TRANSFER COEFFICIENT

B. PROVIDE COOLANT PASSAGES TO MEET THE THERMAL REQUIREMENTS

* FORGING AVAILABLE 

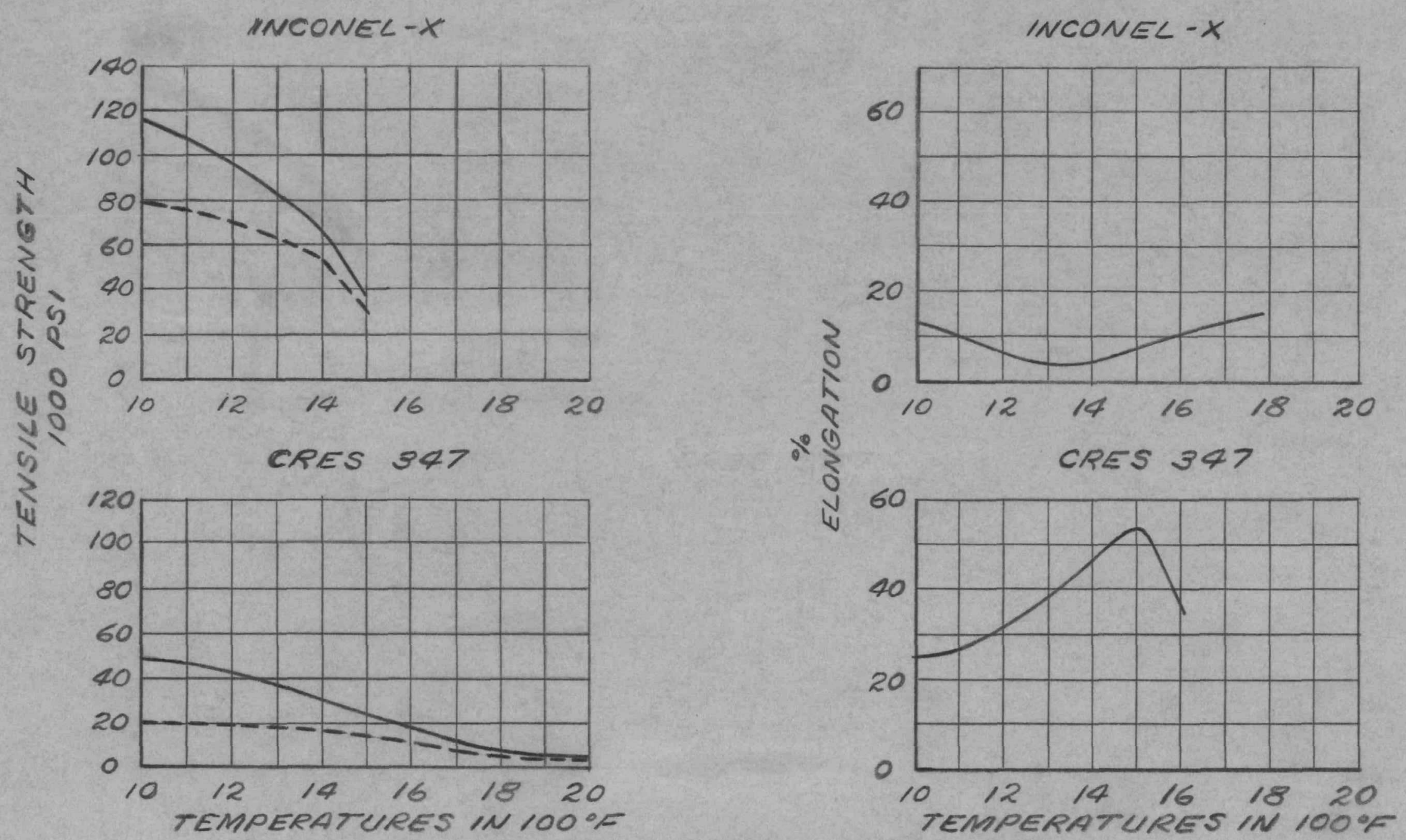


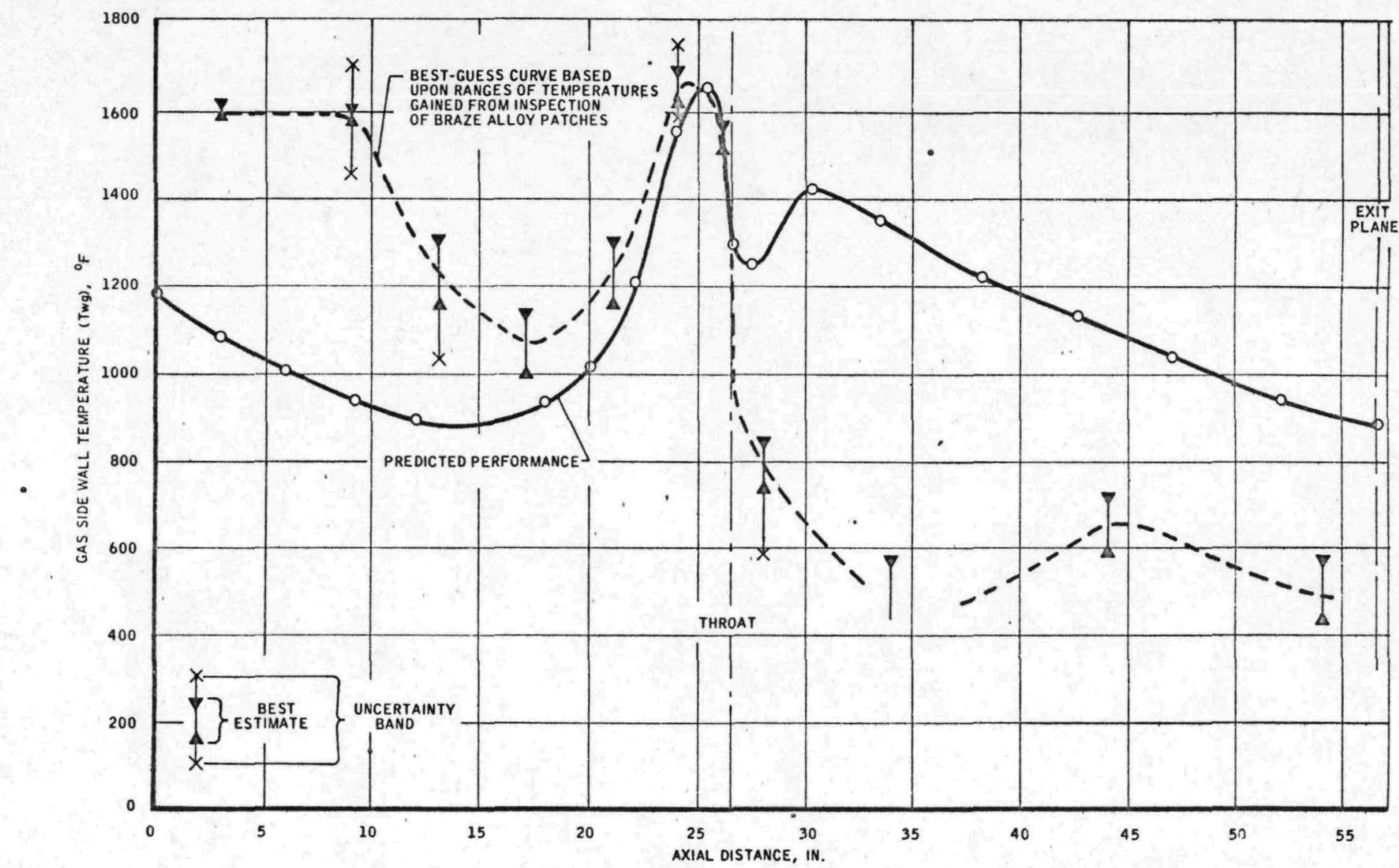


$\mathrm{O}_{2} / \mathrm{H}_{2}$; NOZZLE LIMITS COMPARISON

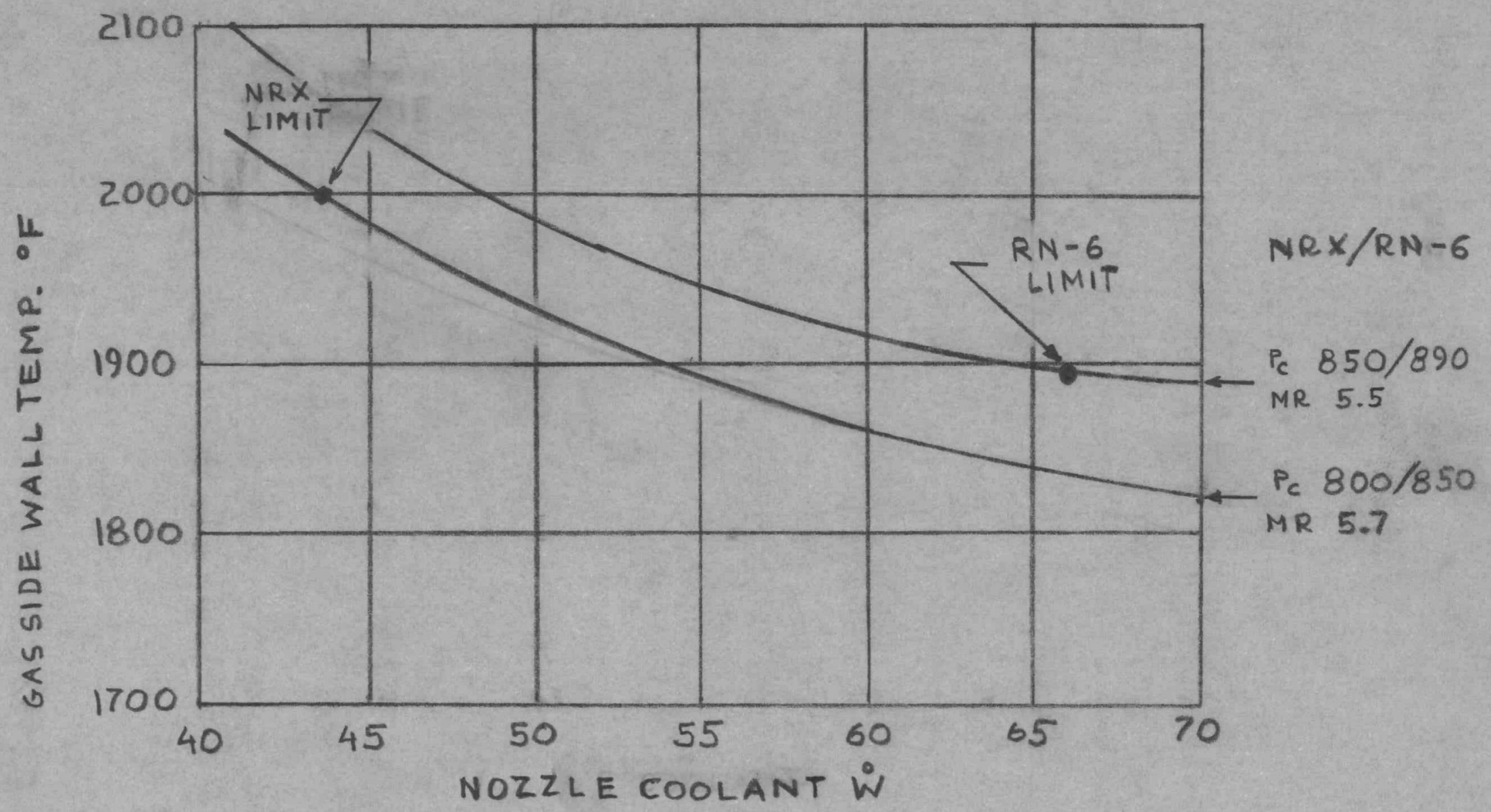




\section{$0_{2} / H_{2}$ TESTS RESULTS}

\begin{tabular}{lccccc} 
& \multicolumn{5}{c}{ NRX NOZZLE } \\
TEST NO. & MR & $P_{C}$ & $\dot{W}_{C}$ & $T_{W G}$ (MAX) CALC. & INDICATED \\
006 & 6.0 & 736 & 71.3 & $1745^{\circ} \mathrm{F}$ & $>1745^{\circ} \mathrm{F}$ \\
007 & 5.8 & 789 & 72.8 & $1810^{\circ} \mathrm{F}$ \\
008 & 5.7 & 801 & 54.6 & $1900^{\circ} \mathrm{F}$ \\
010 & 5.7 & 801 & 43.2 & $1999^{\circ} \mathrm{F}$
\end{tabular}

RN-6 NOZZLE

$\begin{array}{llllcr}317007 & 5.45 & 800 & 66.0 & 1780^{\circ} \mathrm{F} & \\ 317008 & 5.59 & 854 & 66.0 & 1780-1830^{\circ} \mathrm{F} & 1800^{\circ} \mathrm{F} \\ 317008 & 5.65 & 890 & 66.1 & \text { N } / \mathrm{A} & >1800^{\circ} \mathrm{F}\end{array}$


NRX NOZZLE

THROAT DIA. (IN.)

COOLANT FLOW

AREA (IN. ${ }^{2}$ )

TUBE MATERIAL

MELT. TEMP., ${ }^{\circ} R$

8.72

63

CRES 347

$2500-2550$

TUBE WALL

THICKNESS (IN.)

OPERATING AT $1120 \mathrm{MW}_{i} \mathrm{~T}_{\mathrm{C}}=4090^{\circ} \mathrm{R} \quad\left(\mathrm{A}_{\mathrm{C}} / \mathrm{A}_{6}=-1.07\right)$

(Q/A); BTU/IN ${ }^{2}-S E C$

$\mathrm{T}_{W G}{ }^{\circ} \mathrm{F}$

$T_{\text {WALL }}\left(\right.$ MEAN), ${ }^{\circ} \mathrm{F}$

TUBE FAILURE, ${ }^{\circ} \mathrm{F}$

$\left(\mathrm{O}_{2} / \mathrm{H}_{2}\right.$ SYSTEM)
18.15

1570 ( 1620 BLEED PORT)

1180

2000 PLUS
RN-6 NOZZLE

8.72

71

INCO X

$2300-2450$

0.009

17.5

1520

1220

$1800^{\circ}$ PLUS 


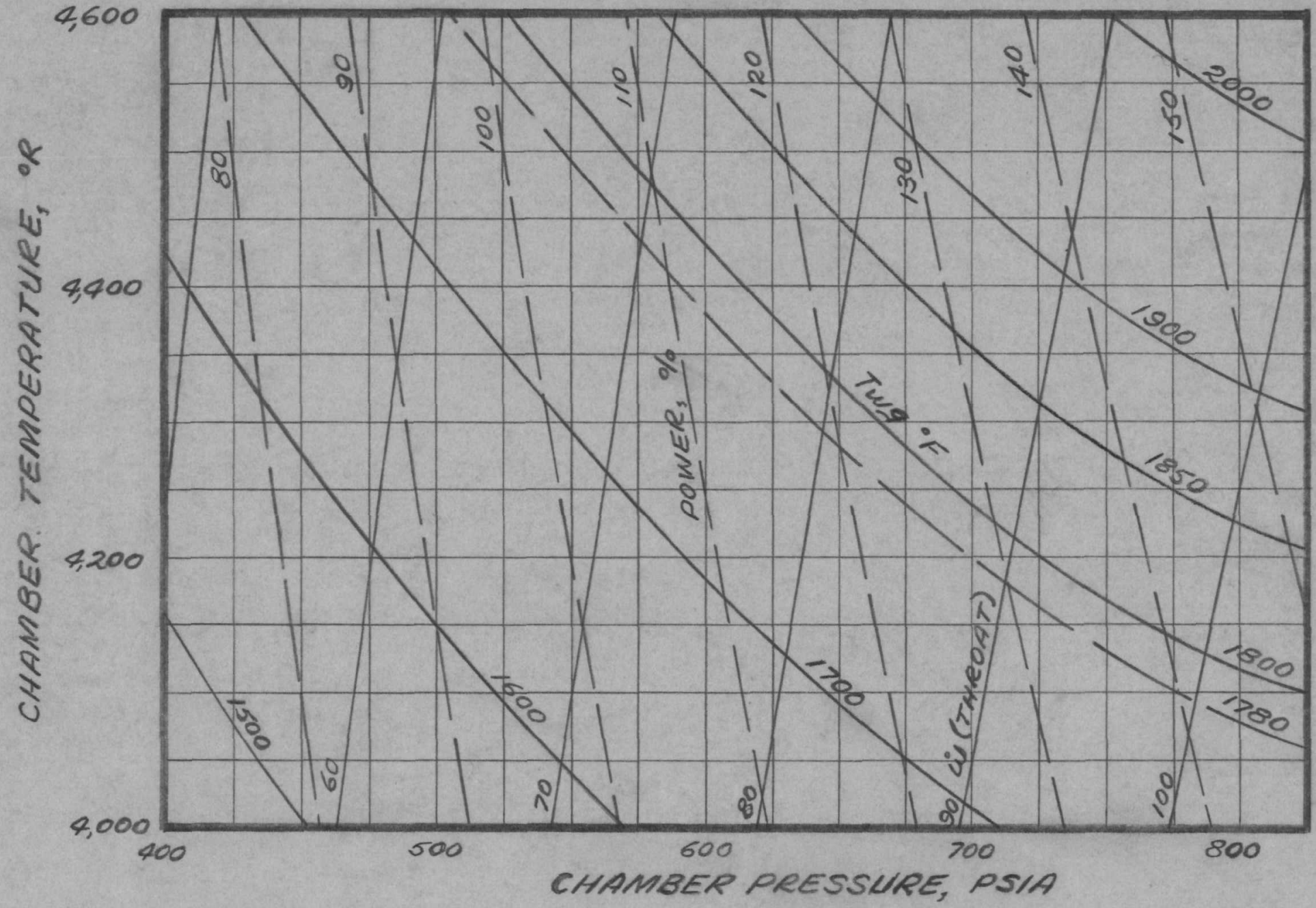



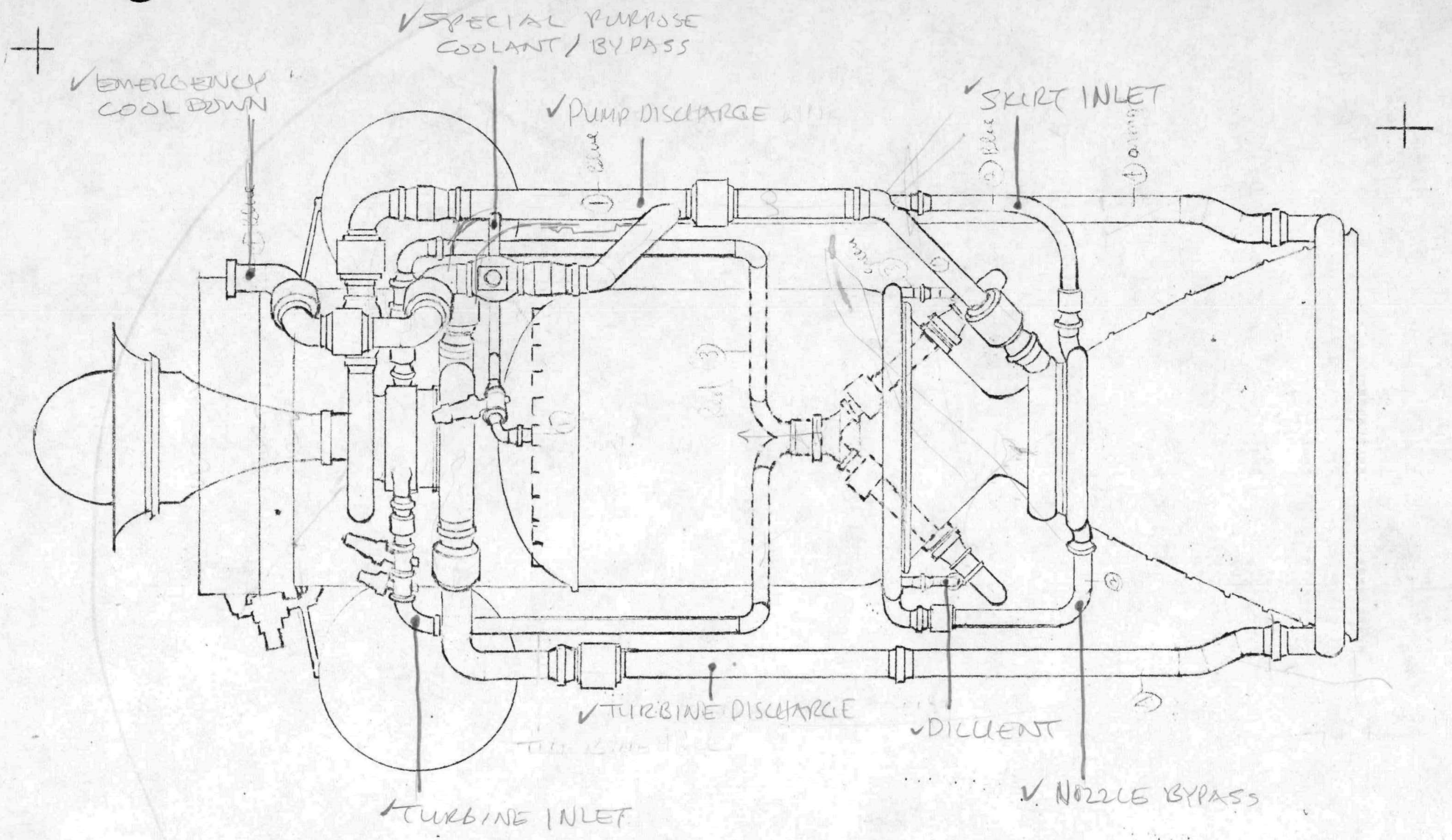

PRELIMINARY NERVA ENGINE ASSEMBLY $\sim 40: 1$ SKIRT 
+ Bree plite-

$$
\begin{aligned}
& \text { Block (line dorjtype) } \\
& \text { (a) }
\end{aligned}
$$

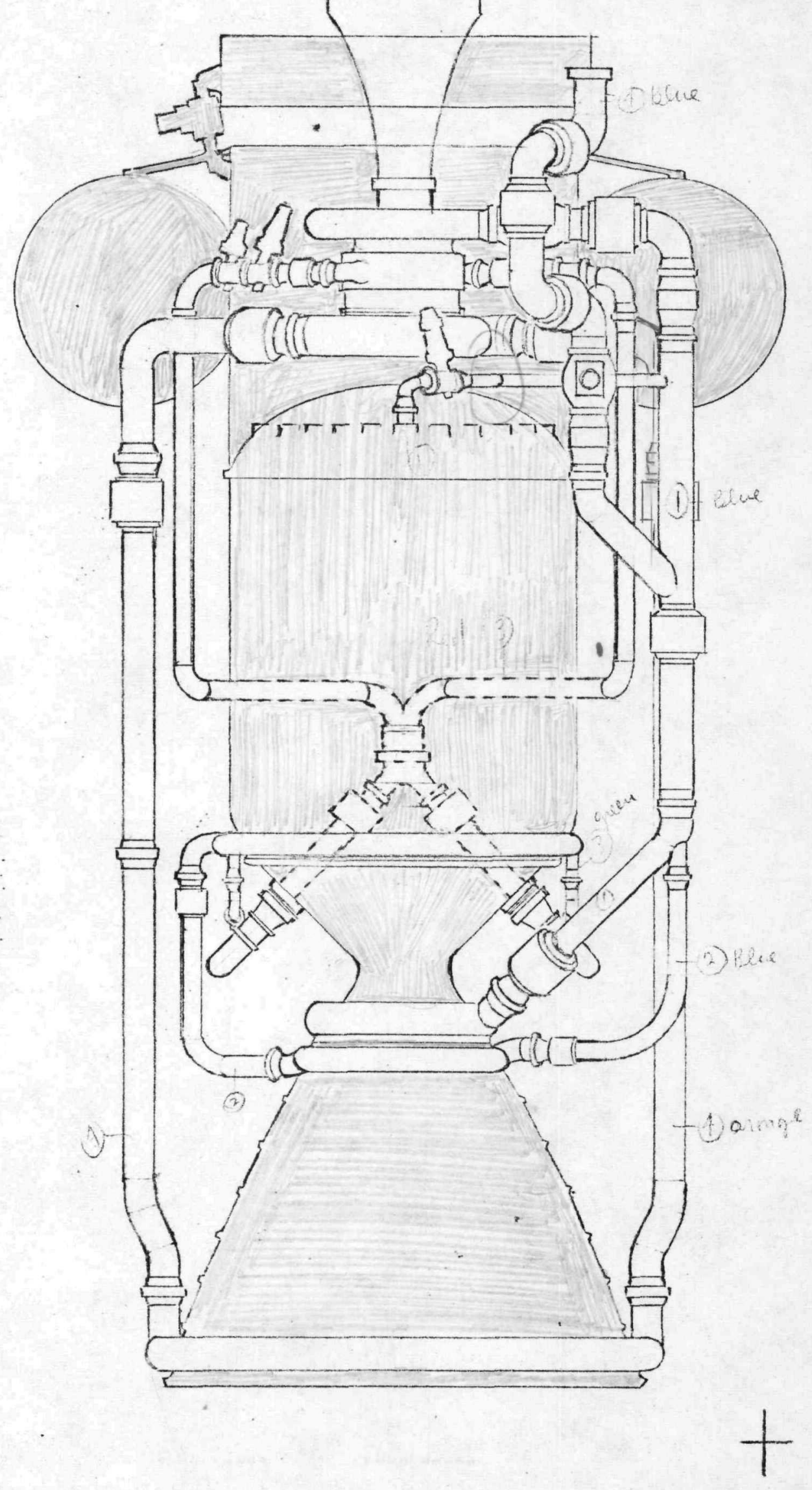


$+\quad b^{\prime} \operatorname{lov} 2$
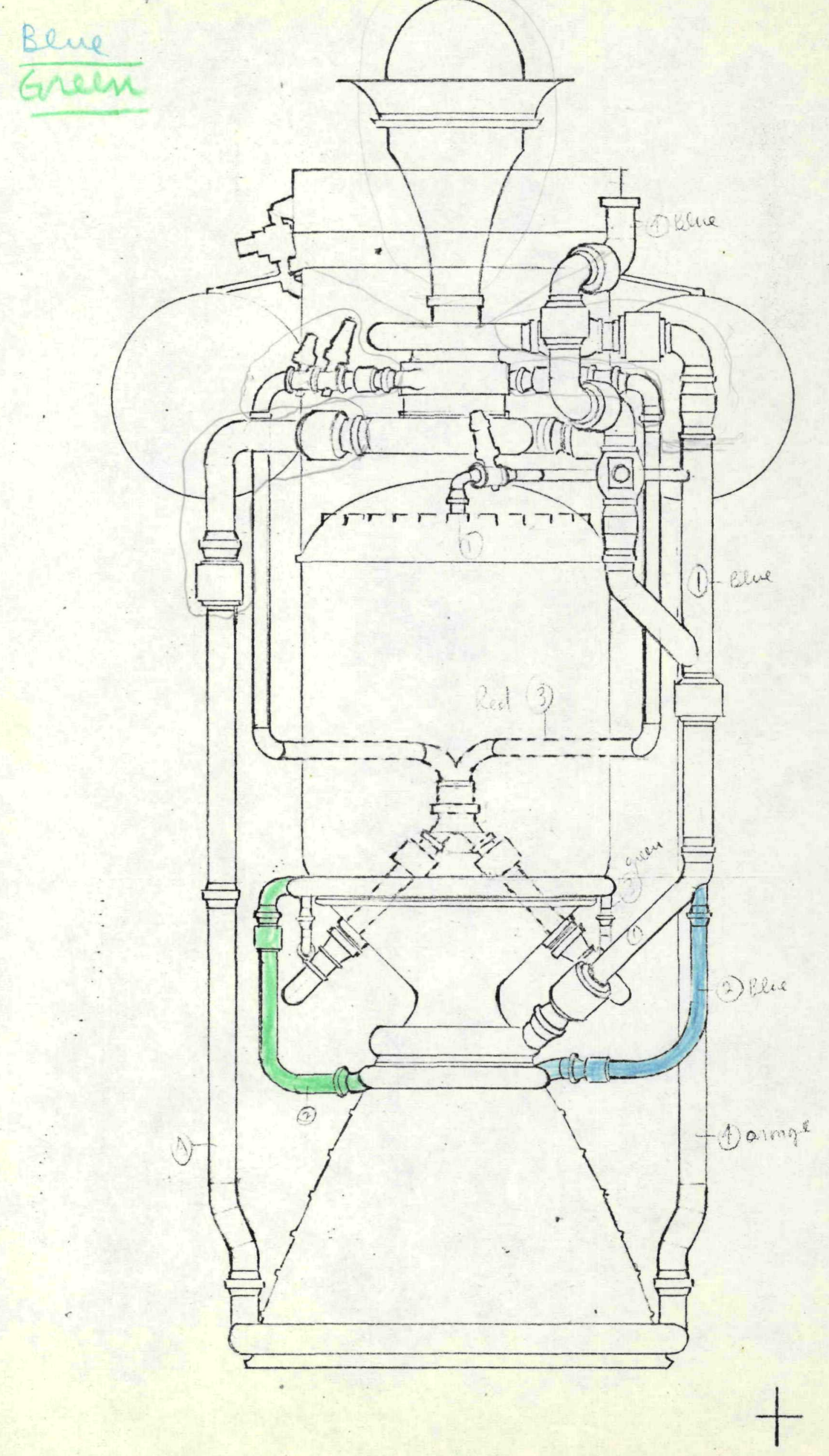
+ oLA 3.
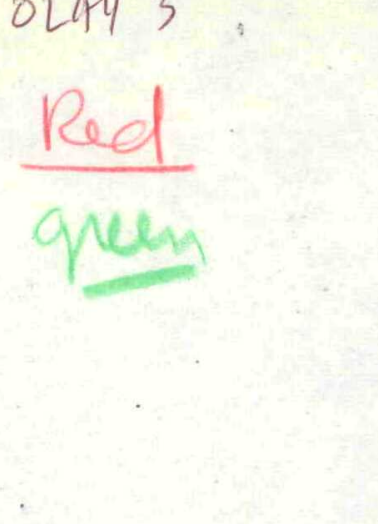

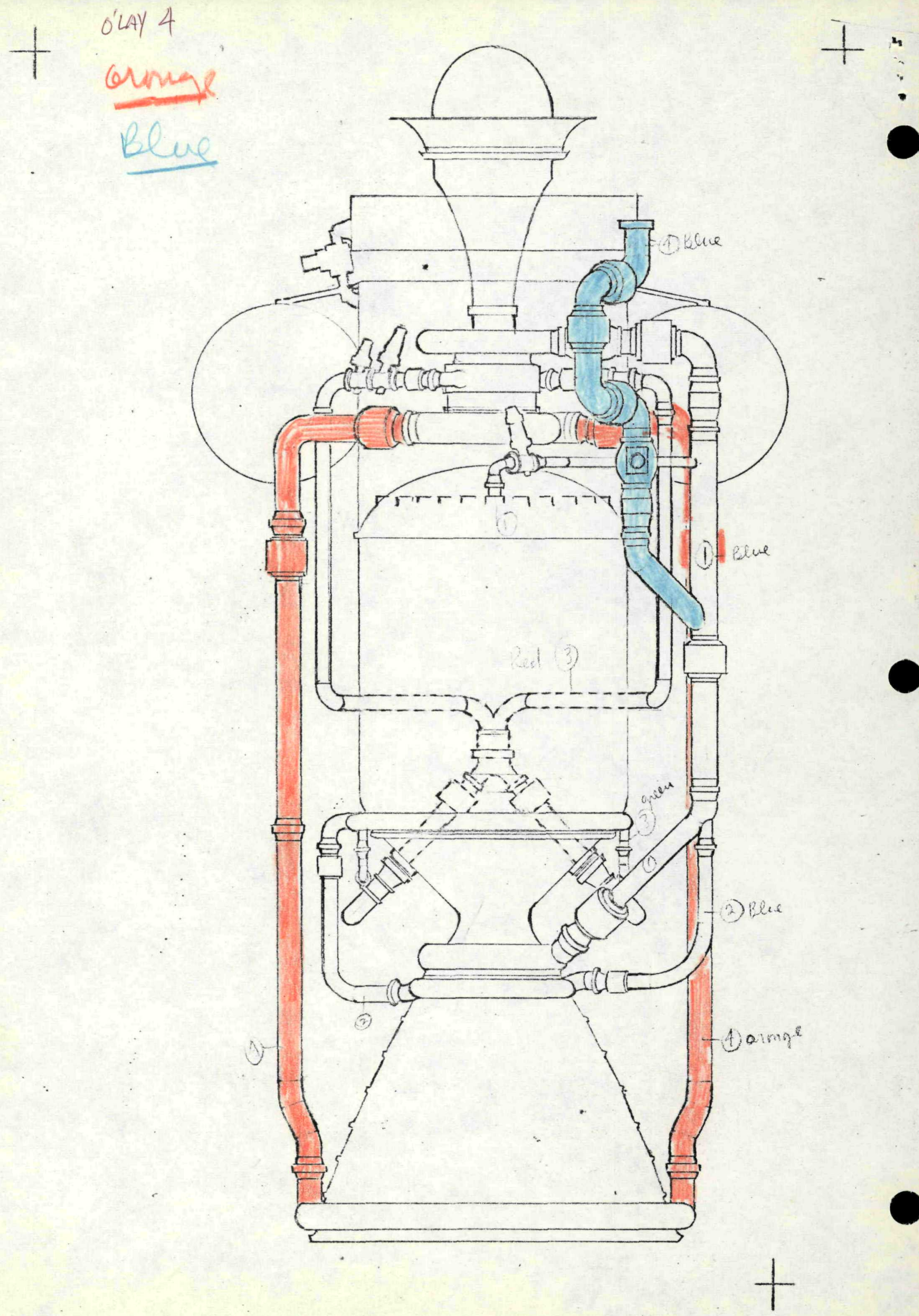


\begin{tabular}{|c|c|c|}
\hline URAD/R & DRAD/R $\mathrm{R}_{\mathrm{t}}$ & $\begin{array}{c}\Delta W \\
(4: 1 \text { NOZZLE ONLY })\end{array}$ \\
\hline 1.0 & 0.2 & BASE CASE \\
\hline 1.0 & 0.5 & 3 LB \\
\hline 1.0 & 1.0 & 6 LB \\
\hline 1.7 & 0.2 & 82 LB \\
\hline 2.0 & 0.2 & 151 LB \\
\hline 3.0 & 0.2 & 194 LB \\
\hline 3.0 & 1.0 & 210 LB \\
\hline
\end{tabular}





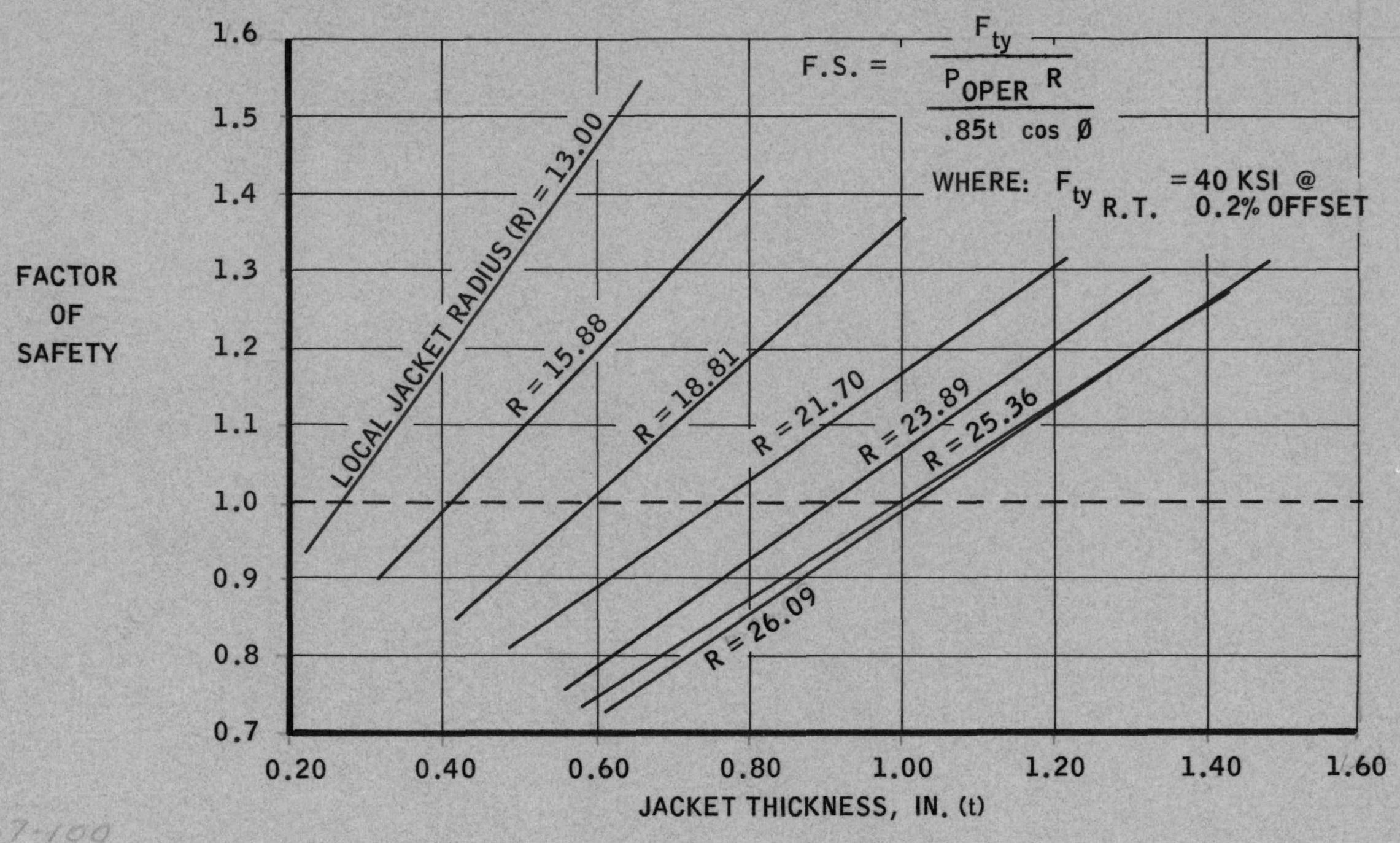




\title{
BLEED PORT:
}

W. HOT GAS

W. DILUENT

W, MIXED GAS

$T$, TURBINE INLET

$\triangle P$, DILUENT

$T$, DILUENT

NO. BLEED PORTS

INSIDE DIAMETER

MATERIAL

\author{
$3.9 \mathrm{LB} / \mathrm{SEC}$ \\ $8.4 \mathrm{LB} / \mathrm{SEC}$ \\ $12.3 \mathrm{LB} / \mathrm{SEC}$ \\ $1660^{\circ} \mathrm{R}$ \\ 160 PSI MIN \\ TO BE DETERMINED \\ 2 \\ 4 IN. \\ TO BE DETERMINED
}


NOZZLE:

$P_{C}$

$T_{C}$

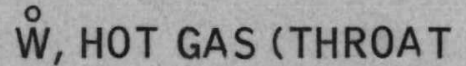

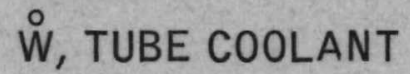

$\stackrel{\text { W, BOLT COOLANT }}{ }$

$\triangle P$, COOLANT

Twg MAX

DIA THROAT

DIA EXIT, 4:1

I.D. CORE SUPPORT FLANGE

MATERIAL

TINLET, COOLANT

THRUST
625 PSIA

$4500^{\circ} \mathrm{R}$

230 LB/SEC

127 LB/SEC

$10 \mathrm{LB} / \mathrm{SEC}$

400 PSID

$1600^{\circ} \mathrm{F}$

15.350 IN.

30.700 IN.

54.500 IN.

HASTELLOY "X"

$55^{\circ}-60^{\circ} R$

$200 \mathrm{~K}$ 
HOT BLEED PORT DESIGN \& FAB

DESIGN \& FAB TEST SUPPORT EQUIPMENT

DESIGN DEVELOPMENT NOZZLE

FAB DEVELOPMENT NOZZLE

REWORK PHOEBUS HARDWARE

CONDUCT INJECTOR DEVELOPMENT TESTS

BLEED PORT/NOZZLE EVALUATION TESTS

NR-I TEST

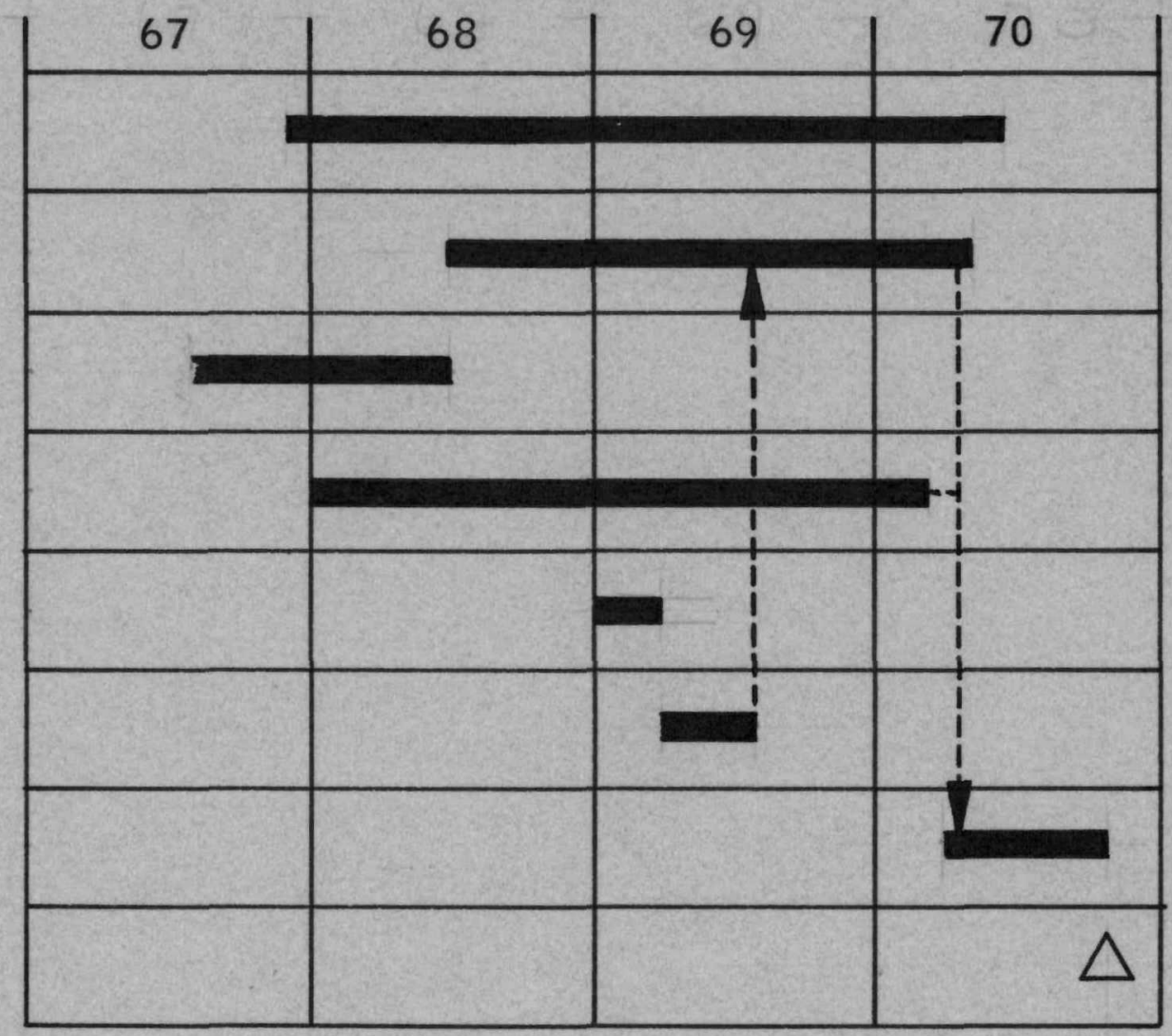


NERVA NOZZLE TWG MAX. VS. COOLANT $\triangle P$
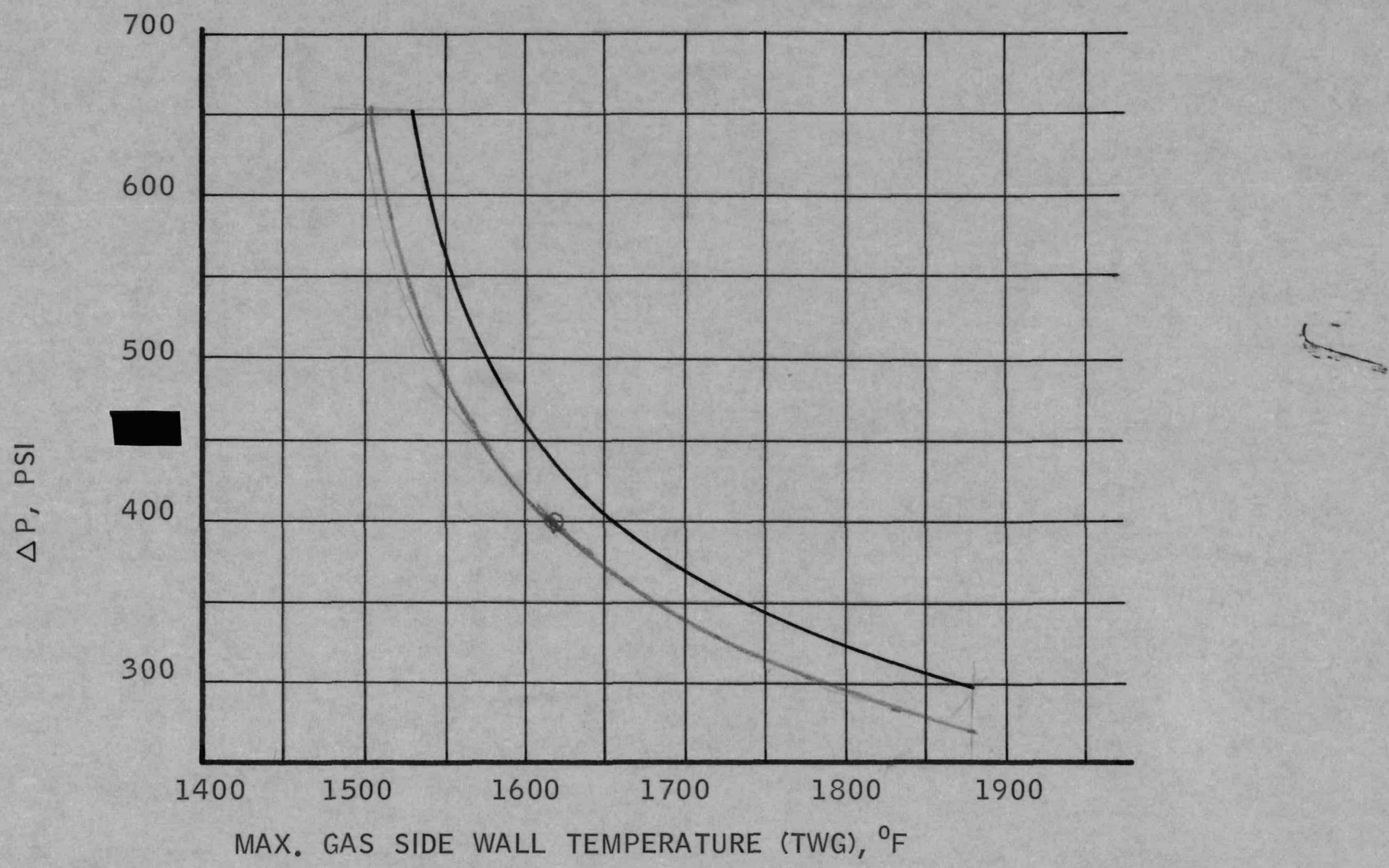
APPLICABLE CONTROLLING DOCUMENTS

DESIGN REQUIREMENTS AND COMPLIANCE WITH

DESIGN CHANGES WITH RESPECT TO NRX/EST

SUITABILITY EVIDENCE DATA

MODES OF FAILURE ANALYSIS

RESIDUAL DISCREPANCIES ACCEPTED 
NDC 123

NDC 117

RN-TM-0159

B/P 1013843

B/P 1114263

B/P 706988

B/P 706967

AGC-90006

AGC-STD-9001

AGC-STD-9007

AGC-46351

AGC-STD-1151

AGC-STD-4005

RN-S-0338

REPORT 2275

XE-1 MRL
$X$-ENGINE COMPONENTS, BASIC DESIGN CRITERIA FOR NOZZLE ASSEMBLY FOR THE XE-1 ENGINE BASIC LOADS AND STRUCTURAL DESIGN CRITERIA FOR THE XE-1 ENGINE INTERFACE CONTROL, XE-1, XE-2 NUCLEAR SUB ASSY (U) NOZZLE, NUCLEAR ROCKET ENGINE (U) NOZZLE, HOT GAS PORT - NUCLEAR ROCKET ENGINE (U) PORT, HOT GAS, NOZZLE (U) NOZZLE, U-TUBE, TUBE INSTALLATION AND BRAZING, PROCEDURE FOR (U) NOZZLE, U-TUBE, NERVA, CLEANING AND PROTECTION OF CLEANLINESS LEVELS, COMPONENTS AND ASSEMBLIES PROCESS SPECIFICATION, FUSION WELDING INSPECTION, RADIOGRAPHIC, MATERIALS, PROCEDURES FOR ENGINEERING ACCEPTANCE CRITERIA FOR WELDS STRUCTURAL ANALYSIS OF THE XE-1 PRESSURE VESSEL COMPONENTS AND ASSEMBLY

MATERIALS PROPERTIES DATA BOOK

MEASUREMENT REQUIREMENTS LIST 


\begin{tabular}{|l|l|}
\hline \multicolumn{1}{|c|}{ REQUIREMENTS } & \multicolumn{1}{c|}{ DEMONSTRATION } \\
\hline $\begin{array}{l}\text { ENVELOPE AND INTERFACES (NDC-117) } \\
\text { APPLICABLE DRAWING } 1013843\end{array}$ & NO DEVIATIONS TO XE ICD \\
\hline $\begin{array}{l}\text { DESCRIPTION } \\
\text { DOCUMENT NO. NDC } 117 \text { PARAGRAPH 1.2 }\end{array}$ & $\begin{array}{l}\text { DWG NO. 1013590-19 \& 706988-29 } \\
\text { NO DEVIATIONS }\end{array}$ \\
\hline $\begin{array}{l}\text { HANDLING - CAPABLE OF POST- } \\
\text { OPERTIVE REMOTE DISASSEMBLY }\end{array}$ & NRX/EST \\
\hline $\begin{array}{l}\text { POSITION INDICATOR } \\
\text { FLUID PORTS: } \\
\text { CLAMP TYPE DESIGN }\end{array}$ & PROVIDED AS PER 706988 \\
\hline $\begin{array}{l}\text { HOT BLEED PORT INSERT ASSY } \\
\text { FLOW REQUIREMENT }\end{array}$ & PROVIDED AS PER 706988 \& 706967 \\
\hline $\begin{array}{l}\text { LEAK CHECK COVER } \\
\text { PROVISION FOR }\end{array}$ & $\begin{array}{l}\text { PROVIDE 1114263-109 } \\
\text { DEMONSTRATED (HYDROTEST) }\end{array}$ \\
\hline
\end{tabular}




\begin{tabular}{|c|c|}
\hline REQUIREMENTS & DEMONSTRATION \\
\hline $\begin{array}{l}\text { APPLIED LOADS PER NDC-117, RN-TM-0159 } \\
\text { NDC-123, RN-S-0231 }\end{array}$ & $\begin{array}{l}\text { 1. ANALYSES DOC. NO. RN-S-0338 } \\
\text { 2. HYDROTEST } \\
\text { 3. NRX/EST } \\
\text { 4. SIMULATION TESTING }\end{array}$ \\
\hline $\begin{array}{l}\text { PRESSURE DROP } \\
170 \text { PSID MAXIMUM }\end{array}$ & $\begin{array}{l}\text { HYDROTEST: REQUIREMENT SATISFIED } \\
\text { (DISCUSSED UNDER FABRICATION) }\end{array}$ \\
\hline $\begin{array}{l}\text { THERMAL SHOCK } \\
\text { SUDDEN DROP FROM } 620 \text { TO } 36^{\circ} \mathrm{R}\end{array}$ & $\begin{array}{l}\text { NOT DEMONSTRATED BEYOND NRX/EST } \\
\text { TEST CONDITIONS }\end{array}$ \\
\hline THERMAL GRADIENTS & NRX/EST \& ANALYSIS RN-S-0338 \\
\hline $\begin{array}{l}\text { BOSSES: STANDARD DIMENSIONS (NDC-123) } \\
\text { THREADED }\end{array}$ & $\begin{array}{l}\text { PROVIDED PER AND } 10050 \\
\text { PROVIDED PER HANDBOOK H28 }\end{array}$ \\
\hline CLEANLINESS & $\begin{array}{l}\text { AGC-STD- } 9007 \text {, LEVELS II \& III } \\
\text { REQUIREMENT SATISFIED } \\
\text { (DISCUSSED UNDER FABRICATION) }\end{array}$ \\
\hline $\begin{array}{l}\text { MARKINGS } \\
\text { PER ASD } 5215 \\
\end{array}$ & DWG 706988 AND 1114263 \\
\hline MATERIAL PROPERTIES & PER DATA BOOK 2275 \\
\hline
\end{tabular}




\begin{tabular}{|c|c|c|}
\hline \multicolumn{2}{|c|}{ REQUIREMENTS } & DEMONSTRATION \\
\hline XE-1 MRL & & PROVIDED FOR PER DWG 706988 \\
\hline \multicolumn{2}{|l|}{ OPERATING PRESSURE } & SIMULATION TEST \\
\hline a. INLET & 905 P SIA & 1080 PSIA \\
\hline b. OUTLET & 735 PSIA & 913 PSIA \\
\hline c. CHAMBER & 561 PSIA & 802 PSIA \\
\hline d. DILUENT INLET & 687 PSIA & 909 P SIA \\
\hline & & * ALSO HYDROSTATIC TESTING \\
\hline
\end{tabular}

* UTILIZING FORMULA:

$$
\text { PROOF = OPERATING PRESSURE }\left(\frac{F_{\text {ty }} \text { RM }}{F_{\text {ty }} \text { OP }}\right) \times 1.05 \text { (MINIMUM) }
$$




\begin{tabular}{|c|c|}
\hline REQUIREMENTS & DEMONSTRATION \\
\hline $\begin{array}{l}\text { LEAKAGE } \\
\text { NDC } 123 \text { PARA } 3.1 .1 .6\end{array}$ & $\begin{array}{l}\text { SATISFIED THE REQUIREMENT USING } \\
4 \text { TIMES THE SPEC PRESSURE } \\
\text { (ALSO DISCUSSED UNDER FAB HISTORY }\end{array}$ \\
\hline $\begin{array}{l}\text { NONMETALS } \\
\text { (RESTRICTIONS) }\end{array}$ & NONE USED \\
\hline $\begin{array}{l}\text { PROTECTIVE TREATMENT } \\
\text { (RESTRICTIONS) }\end{array}$ & NONE USED \\
\hline $\begin{array}{l}\text { NON-OPERATING CONDITIONS } \\
\text { NDC } 123 \quad 3.2 .2 .2 \text { (a) THROUGH (i) }\end{array}$ & $\begin{array}{l}\text { THE ASSEMBLY IS NOT SUSCEPTIBLE } \\
\text { TO DAMAGE UNDER CONDITIONS STATED } \\
\text { (NO DEMONSTRATION WAS CONDUCTED) }\end{array}$ \\
\hline SERVICE LIFE & DEMONSTRATED (NRX-A5, EST) \\
\hline STORAGE LIFE & DEMONSTRATED (S/N 025) \\
\hline $\begin{array}{l}\text { EXPOSURE TO REACTOR EXIT GASES: } \\
\text { CAPABILITY OF: } \\
\text { a. NOZZLE WALL THROAT/EXIT } \\
1620 / 700^{\circ} \mathrm{F} \\
\text { b. BLD PORT ON SPACERS/BTW SPACERS } \\
1460 / 1360^{\circ} \mathrm{F}\end{array}$ & $\begin{array}{l}\text { DEMONSTRATED (CHEM SIM.) } \\
1900 / 460^{\circ} \mathrm{F} \\
1760 / 1580^{\circ} \mathrm{F}\end{array}$ \\
\hline
\end{tabular}




\begin{tabular}{|c|c|}
\hline REQUIREMENTS & DEMONSTRATION \\
\hline $\begin{array}{l}\text { CAPABLE TO WITHSTAND SELF INDUCED } \\
\text { VIBRATION }\end{array}$ & $\begin{array}{l}\text { ANALYSES (RN-TM-0281), } \\
\text { NRX/EST, CHEM. SIM. }\end{array}$ \\
\hline CAPABLE TO WITHSTAND GAMMA HEATING & $\begin{array}{l}\text { ANALYSES }(7730: 0522 \mathrm{M}) \\
\text { JACKET ANALYZED TO } 1500 \mathrm{MW}(1.5) \\
\text { LIMITING } P_{C} \text { TO } 750 \text { PSIA }\end{array}$ \\
\hline 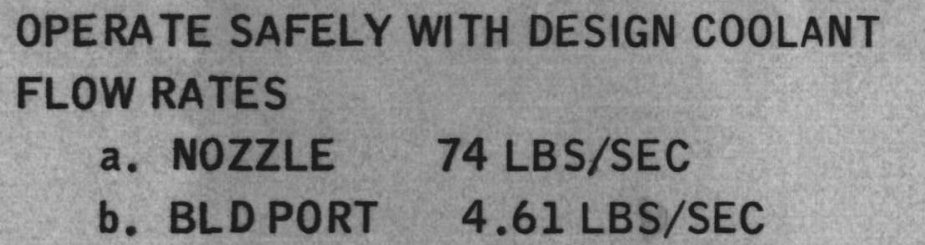 & $\begin{array}{l}\text { DEMONSTRATED (CHEM SIM.) } \\
54.6 \text { LBS/SEC } \\
2.51 \text { LBS/SEC }\end{array}$ \\
\hline PROVIDE AND OPERATE AT $T_{I T}$ OF $1184^{\circ} \mathrm{R}$ & $1608^{\circ} \mathrm{R}$ \\
\hline
\end{tabular}


SUMMARY OF TEST CONDITIONS FOR TEST SERIES

\begin{tabular}{|c|c|c|c|c|c|c|c|}
\hline \multirow[b]{2}{*}{$\begin{array}{l}\text { TEST } \\
\text { NO. }\end{array}$} & \multirow[b]{2}{*}{$\begin{array}{l}\text { DURATION } \\
\text { (SEC) }\end{array}$} & \multirow{2}{*}{$\begin{array}{c}\text { STEADY } \\
\text { STATE } \\
\text { DURATION } \\
\text { (SEC) }\end{array}$} & \multirow{2}{*}{$\begin{array}{l}\text { CHAMBER } \\
\text { PRESSURE } \\
\text { (PSIA) }\end{array}$} & \multicolumn{2}{|c|}{ MIXTURE RATIO } & \multirow{2}{*}{$\begin{array}{c}\text { NOZZLE } \\
\text { COOLANT } \\
\text { FLOW (\#/SEC) }\end{array}$} & \multirow{2}{*}{$\begin{array}{l}\text { MAXIMUM PREDICTED } \\
\text { HOT-GAS-SIDE TUBE } \\
\text { WALL TEMP }\left({ }^{\circ} \mathrm{F}\right)\end{array}$} \\
\hline & & & & NOMINAL & $\begin{array}{l}\text { BETWEEN } \\
\text { BAFFLES }\end{array}$ & & \\
\hline 001 & 15.1 & 7.8 & 674 & 4.7 & 5.8 & 72.9 & 1648 \\
\hline 002 & 1.7 & 0 & 437 & 1.2 & 1.5 & 75.0 & $\ldots$ \\
\hline 003 & 6.5 & 0 & 721 & 3.6 & 4.5 & 77.1 & ---- \\
\hline 004 & 3.5 & 0 & 661 & 2.8 & 3.5 & 74.0 & $\cdots--$ \\
\hline 005 & 5.8 & 0 & 645 & 5.0 & 6.2 & 56.7 & $\cdots--$ \\
\hline 006 & 20.8 & 13.3 & 736 & 4.85 & 6.0 & 71.3 & 1745 \\
\hline 007 & 20.5 & 11.5 & 789 & 4.66 & 5.8 & 72.8 & 1806 \\
\hline 008 & 20.8 & 12.0 & 801 & 4.63 & 5.7 & 54.6 & 1900 \\
\hline 009 & 1.3 & 0 & -- & $--\cdot$ & $--\cdot$ & $\ldots$ & $\cdots--$ \\
\hline 010 & 20.4 & 11.5 & 801 & 4.63 & 5.7 & 43.2 & 1997 \\
\hline
\end{tabular}




\begin{tabular}{|c|c|c|c|c|c|c|c|c|c|c|c|c|}
\hline \multirow{3}{*}{$\begin{array}{l}\text { TCA } \\
S / N\end{array}$} & \multirow{3}{*}{$\begin{array}{l}\text { TEST } \\
\text { NO. }\end{array}$} & \multirow{3}{*}{$\begin{array}{c}\mathrm{P}_{\mathrm{C}} \\
\text { (PSIA) }\end{array}$} & \multirow{3}{*}{ MR } & \multirow{3}{*}{$\begin{array}{l}\dot{W}_{H . G .} \\
\text { (LB/SEC) }\end{array}$} & \multirow{3}{*}{$\begin{array}{l}\dot{W}_{\text {DIL }} \\
\text { (LB/SEC, } \\
\text { EXCLUDING } \\
\text { BYPASS) }\end{array}$} & \multirow{3}{*}{$\begin{array}{l}\% \text { DIL } \\
\text { BYPASS }\end{array}$} & \multirow{3}{*}{$\begin{array}{c}\text { DIL } \\
\Delta P \\
(P S I)\end{array}$} & \multirow{3}{*}{$\begin{array}{l}\text { TURBINE } \\
\text { INLET } \\
\text { TEMP }\left({ }^{\circ} R\right)\end{array}$} & \multicolumn{4}{|c|}{ BLEED PORT LINER MAX WALL TEMP $\left({ }^{\circ} R\right)$} \\
\hline & & & & & & & & & \multicolumn{2}{|c|}{ COMPUTER PREDICTED } & \multicolumn{2}{|c|}{ MEAS. BY BRAZE ALLOYS } \\
\hline & & & & & & & & & \begin{tabular}{c|} 
OVER \\
SPACERS
\end{tabular} & $\begin{array}{l}\text { BETWEEN } \\
\text { SPACERS }\end{array}$ & $\begin{array}{c}\text { OVER } \\
\text { SPACERS }\end{array}$ & $\begin{array}{l}\text { BETWEEN } \\
\text { SPACERS }\end{array}$ \\
\hline 013 & 010 & 563 & 5.27 & 5.06 & 3.40 & 0 & 101 & 1597 & 2156 & 1968 & - & 2035 \\
\hline 013 & 013 & 567 & 5.19 & 5.87 & 2.51 & 26.4 & 31 & 1608 & 2311 & 2202 & - & - \\
\hline 021 & 006 & 645 & 4.72 & 6.40 & 4.53 & 14.1 & 72 & 1235 & 2152 & 1920 & $>2157$ & $<1960$ \\
\hline 021 & 007 & 638 & 4.72 & 5.55 & 4.72 & 14.1 & 93 & 1157 & 2101 & 1851 & - & - \\
\hline 021 & 010 & 652 & 4.59 & 5.97 & 4.23 & 14.1 & 62 & 1387 & 2184 & 1978 & $>2157$ & $<1960$ \\
\hline 027 & 001 & 679 & 4.71 & 6.44 & $3.47 \star$ & 8.3 & 54 & 1590 & 2232 & 2047 & - & - \\
\hline 027 & 006 & 736 & 4.85 & 5.51 & 5.72 & 8.3 & 60 & 1250 & 2053 & 1790 & - & - \\
\hline 027 & 007 & 789 & 4.66 & 5.64 & 5.65 & 8.3 & 52 & 1270 & 2053 & 1792 & - & - \\
\hline 027 & 008 & 801 & 4.63 & 4.76 & 6.02 & 8.3 & 60 & 1280 & 1970 & 1709 & - & - \\
\hline 027 & 010 & 801 & 4.63 & 4.85 & 5.52 & 8.3 & 62 & 1550 & 1997 & 1735 & - & - \\
\hline \multicolumn{2}{|c|}{$\begin{array}{l}\text { XE DESIGN } \\
\left(4090^{\circ} \mathrm{R} \quad \mathrm{T}_{\mathrm{c}}\right)\end{array}$} & 563 & - & 1.60 & 4.61 & 14.4 & 90 & 1140 & 1921 & 1723 & - & - \\
\hline
\end{tabular}

* DILUENT FLOW WAS RESTRICTED ON THIS TEST DUE TO ICE FORMING IN THE DILUENT MIXER UPSTREAM OF THE HOT BLEED PORT DILUENT INLET, 
1. BOLT COOLANT CIRCUIT

2. COOLANT HOLES AROUND BLEED PORT

3. BLEED PORT TO NOZZLE SEAL

4. AFT CLOSURE FLANGE

5. INSTRUMENTATION 


\begin{tabular}{|l|c|c|}
\cline { 2 - 3 } \multicolumn{1}{c|}{} & NRX/EST & XE \\
\hline DESIGN FLOW RATE (LB/SEC) & 5.00 & 3.00 \\
\hline VENTURI THROAT DIAMETER (INCH) & .450 & .300 \\
\hline ORIFICE DIAMETER (INCH) & $\begin{array}{l}.367 \\
\text { LINE ATTACHMENT MESHED }\end{array}$ & $\begin{array}{l}\text { THREADED } \\
\text { FITTING }\end{array}$ \\
\hline BOLT BYPASS CIRCUIT & YES & WELD-ON \\
\hline
\end{tabular}




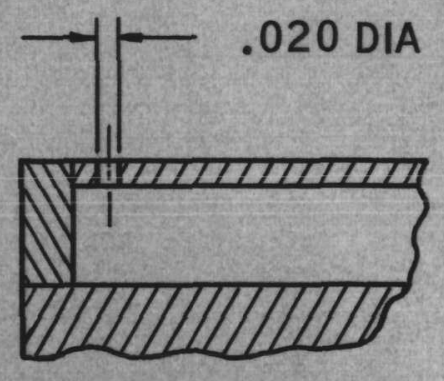

$.070 \% .080$ PRIOR TO MACHINING $.063 / .074$ AFTER MACHINING

EST

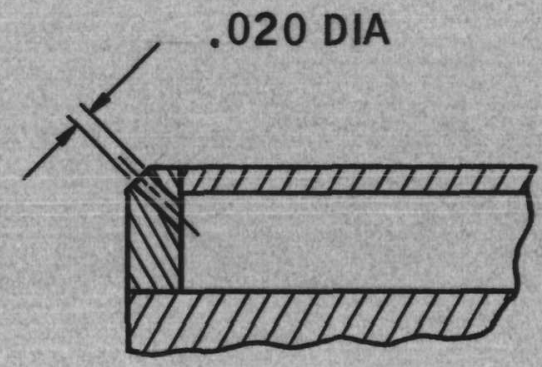

$.100 / .110$ PRIOR TO MACHINING $.084 / .095$ AFTER MACHINING

XE

SUBJECT TO LAYUP TOLERANCES 

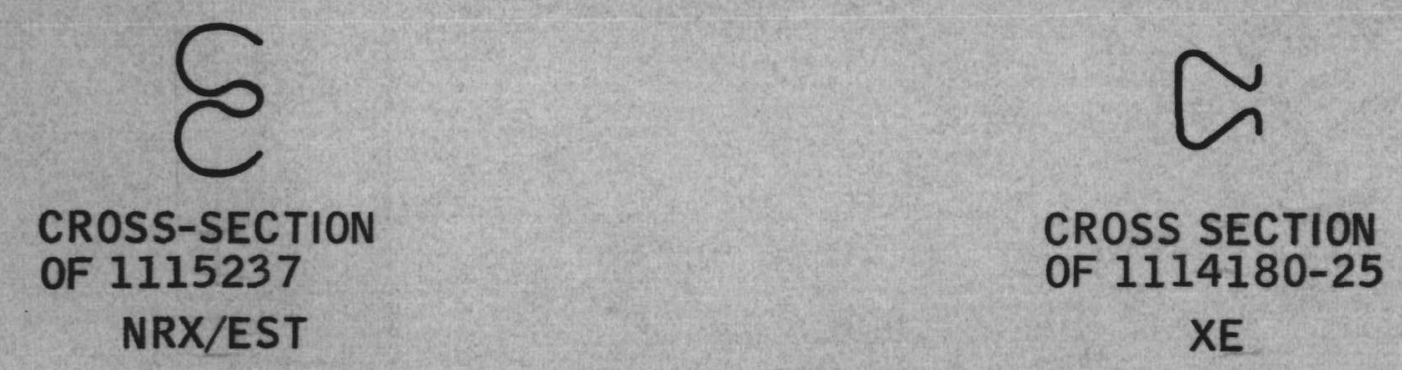

PRESSURE DECAY IN 5 MIN. FROM 800 PSIG USING HELIUM

\begin{tabular}{|c|c|c|}
\hline S/N & CYCLES & DECAY (PSI) \\
\hline 17 & 1 & 115 \\
& 2 & 98 \\
& 3 & 80 \\
\hline \multirow{2}{*}{18} & 1 & 20 \\
& 2 & 17 \\
& 3 & 14 \\
\hline
\end{tabular}

*USED SEAL

**INTERFERENCE IN SEAL TESTER

\begin{tabular}{|c|c|c|}
\hline S/N & CYCLES & DECAY (PSI) \\
\hline 374 & 1 & 3 \\
& 2 & 3 \\
& 3 & 5 \\
\hline 375 & 1 & 4 \\
& 2 & 5 \\
& 3 & 3 \\
\hline 378 & 1 & 13 \\
& 2 & 5 \\
& 3 & 3 \\
\hline$* 380$ & 1 & 2 \\
& 2 & 6 \\
& 3 & 11 \\
$* 383$ & 1 & 19 \\
\hline
\end{tabular}



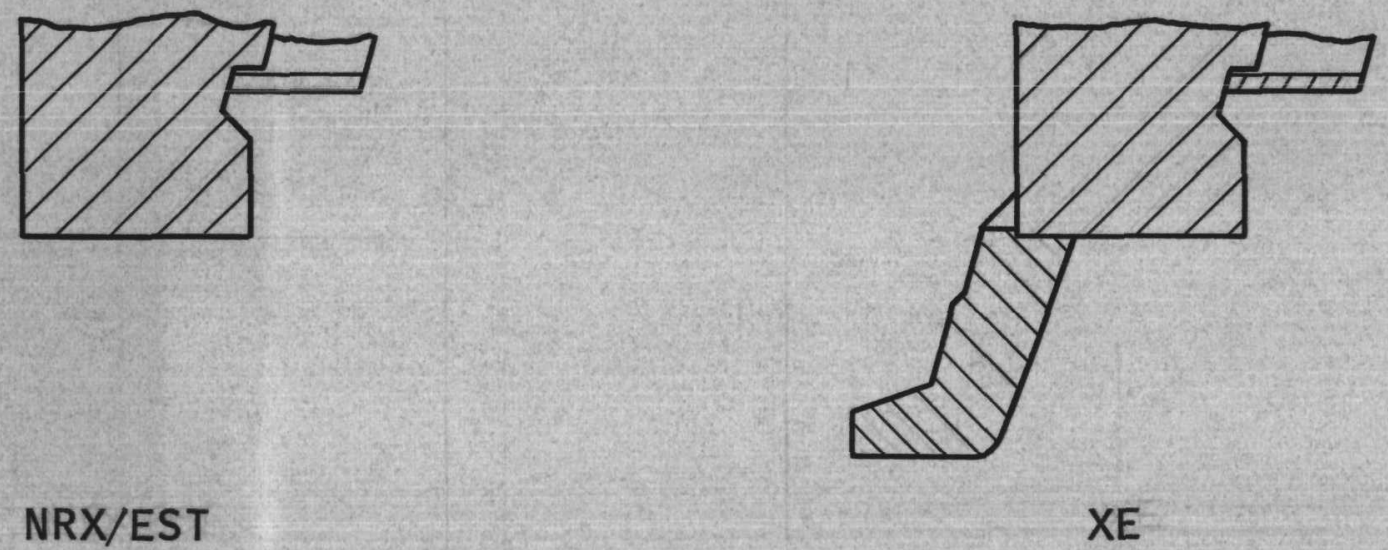

NRX/EST

ADDED FLANGE AS REQUIRED PER PARA 3.1.2.6 OF NDC 117 
NOZZLE RESONANT FREQUENCY (BELL MODE)

ACCELERATION, 0 TO PEAK, $g$ 's (NRX/EST)

AT $235-240 \mathrm{cps}$

FROM XECF VIBRATION ANALYSES, XE NOZZLE WILL SEE 3 TIMES THAT OF NRX/EST AT

235-240 cps

SEEN DURING CHEMICAL SIMULATION TEST

AT $235-240 \mathrm{cps}$

FROM RN-TM-0281 NOZZLE IS CAPABLE OF

AT $235-240 \mathrm{cps}$
$235-240 \mathrm{cps}$

$\pm 3.5$

$\pm 10.5$

$\pm 15.0$

$\pm 353$ 


$$
K_{w}=\frac{\dot{w}}{\sqrt{\Delta P S \cdot G}}
$$

$\mathrm{K}_{w}=$ FLOW COEFFICIENT

$\dot{W}=$ TOTAL FLOW RATE

$\triangle P=P R E S S U R E D R O P$

S.G. = SPECIFIC GRAVITY ( = I FOR WATER)

\begin{tabular}{|l|c|c|c|}
\cline { 2 - 4 } \multicolumn{1}{c|}{} & $K_{W}$ & $\dot{W}_{T}$ & $\Delta P$ \\
\hline NRX/EST & 29.47 & 76.2 & 165 \\
\hline$X E$ & 29.69 & 77.0 & 166 \\
\hline
\end{tabular}

MAXIMUM XE PRESSURE DROP IS 170 PSI AS STATED PREVIOUSLY 
NRX/EST (S/N 026)

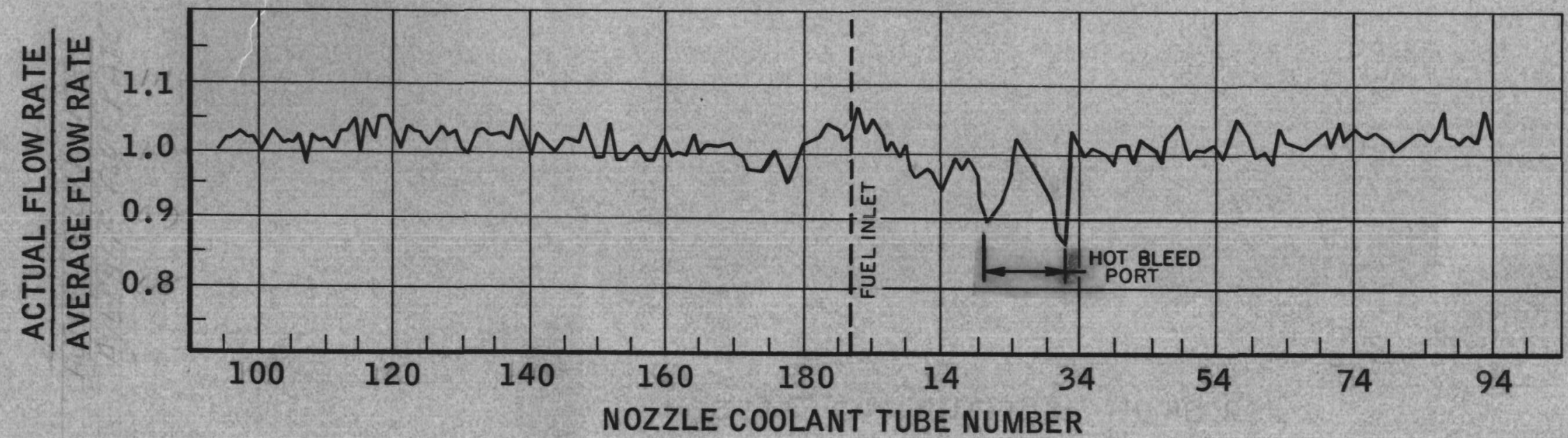

XE (S/N 030)

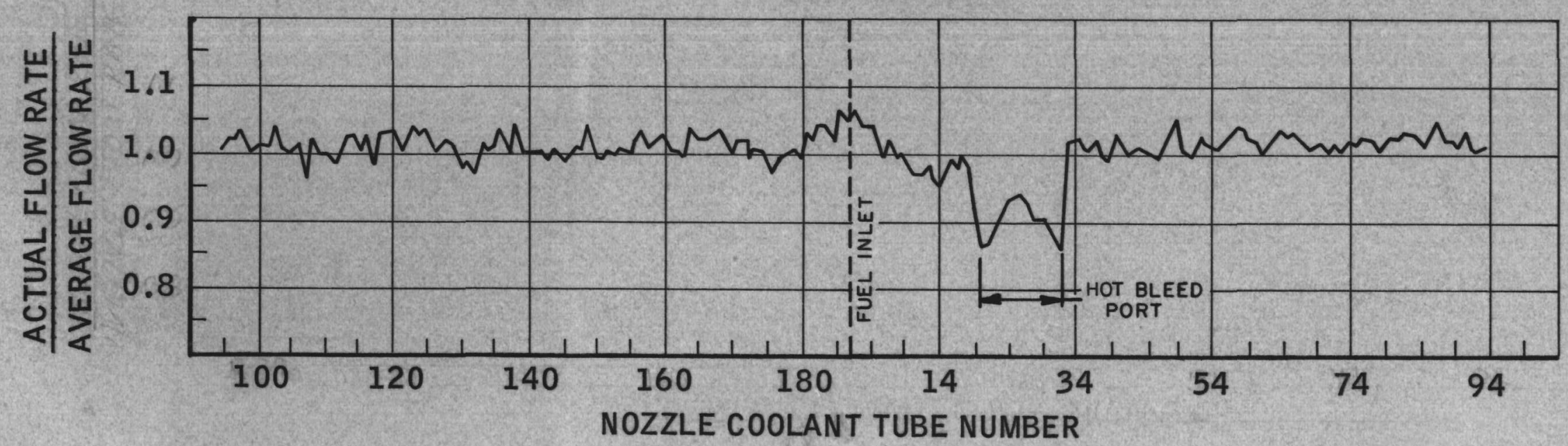


AFTER BACKFLUSH, RADIOGRAPHIC INSPECTION PER AGC-STD-1151

A. ATTACHED PARTICLES IN THE TORUS ONLY ARE ACCEPTABLE IF THEY DO NOT PROTRUDE INTO COOLANT PASSAGEWAY OVER .12 IN.

B. LOOSE OR ATTACHED PARTICLES OTHER THAN ABOVE ARE UNACCEPTABLE.

REMAINING INDICATIONS ACCEPTED ON I.R. 420720 
PROOF AND LEAK TEST WITH GN 2 :

PROOF PRESSURE $=200$ PSIG

LEAK PRESSURE $=100 \mathrm{PSIG}$

NO BUBBLES WHEN SOLUTION PER MIL-L-25567 IS APPLIED

PROOF AND LEAK TEST WITH WATER:

PROOF PRESSURE $=1150$ PSIG

LEAK PRESSURE $=860 \mathrm{PSIG}$

NO VISUAL DEFORMATION OF LEAKAGE ALLOWED

PROOF AND LEAK TEST WITH HELIUM:

PROOF PRESSURE $=200$ PSIG

LEAK PRESSURE $=100 \mathrm{PSIG}$

NO LEAKAGE ALLOWED AS SHOWN BY BUBBLES

IN SOLUTION PER MIL-L-25567 
PROOF AND LEAK TEST WITH WATER:

PROOF PRESSURE $=800$ PSIG

LEAK PRESSURE $=600 \mathrm{PSIG}$

NO VISUAL DEFORMATION OF LEAKAGE ALLOWED

ICD MEASUREMENTS CHECKED FOLLOWING THIS TEST

PER RN-S-0338, THIS TEST IS MOST CRITICAL IN PRIMARY STRESS

PROOF AND LEAK TEST WITH HELIUM:

PROOF PRESSURE $=200$ PSIG

LEAK PRESSURE $=100 \mathrm{PSIG}$

NO LEAKAGE AT SEAL BETWEEN BLEED PORT AND NOZZLE WHEN CHECKED WITH SOLUTION PER MIL-L-25567 

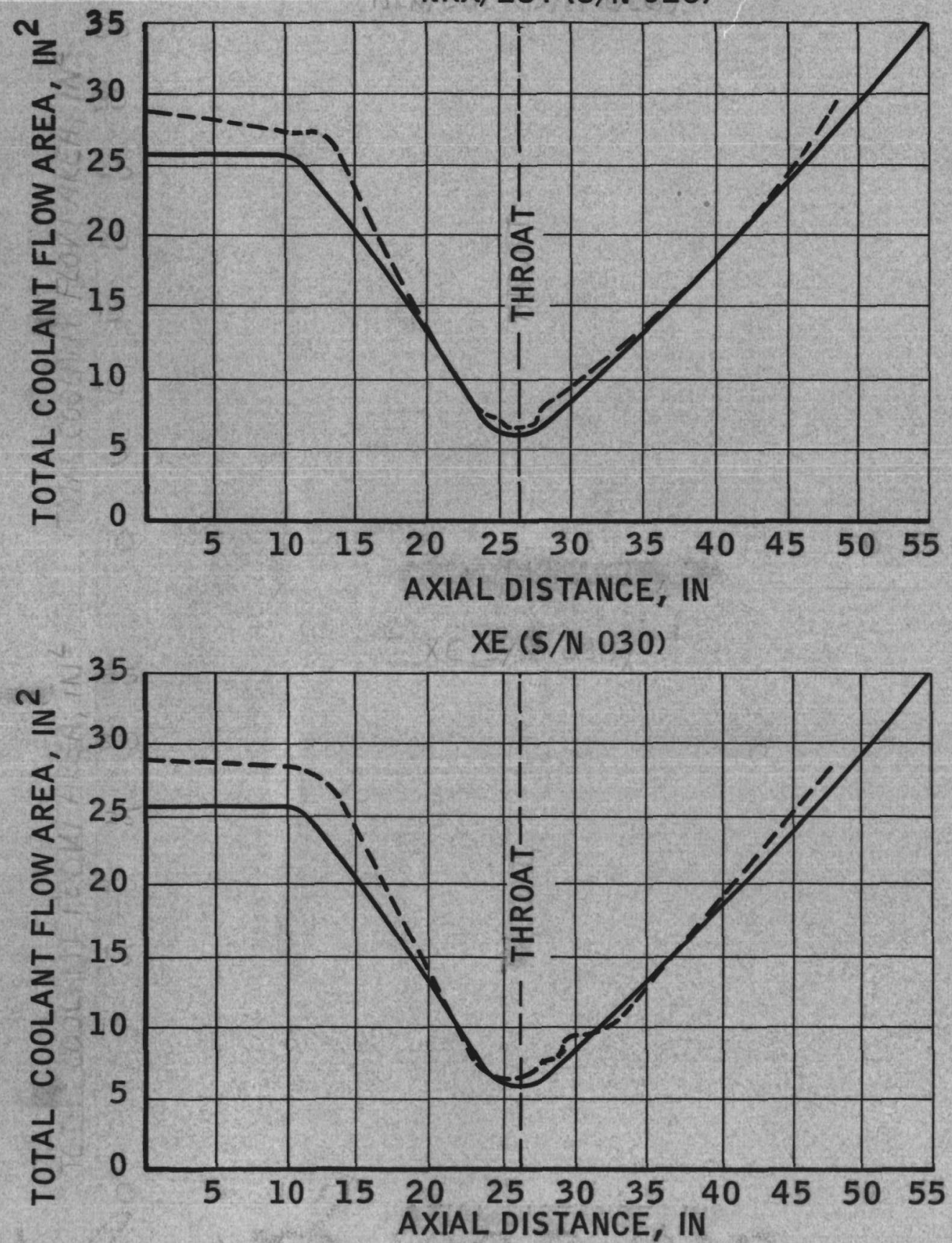


\begin{tabular}{|l|c|c|c|}
\cline { 2 - 4 } \multicolumn{1}{c|}{} & $\begin{array}{c}\text { CHEMICAL SIMULATION } \\
\text { S/N O21 SERIES } \\
\text { TEST 010 }\end{array}$ & NRX/EST & XE \\
\hline WATER K FACTOR & 7.18 & 7.03 & 6.98 \\
\hline TOTAL DILUENT FLOW (LB/SEC) & 4.93 & 5.59 & 5.39 \\
\hline PERCENT OF FLOW THROUGH LINER & 85.9 & 89.4 & 85.6 \\
\hline FLOW THROUGH LINER & 4.23 & 5.00 & 4.61 \\
\hline
\end{tabular}


CLEAN NOZZLE PER AGC-STD-9001 METHODS 6, 7, AND 8

ACCEPTANCE CRITERIA PER AGC-STD-9007

EXTERIOR SURFACES - LEVEL II

INTERNAL FLOW PASSAGE - LEVEL III

ACCEPTABLE AS SHOWN BY PARTICLE COUNT. BOUGHT-OFF BY PROCESS CONTROL LAB AND INSPECTION. 


\begin{tabular}{|c|c|c|c|c|}
\hline & $\begin{array}{l}\text { YIELD STRENGTH } \\
(.2 \% \text { OFFSET), PSI } \\
\end{array}$ & $\begin{array}{c}\text { ULTIMATE STRENGTH } \\
\text { PSI }\end{array}$ & $\begin{array}{c}\text { ELONGATION } \\
\% \\
\end{array}$ & $\begin{array}{c}\text { REDUCTION OF AREA } \\
\%\end{array}$ \\
\hline REQUIREMENT & 30,000 & 75,000 & 40 & 50 \\
\hline \multirow{2}{*}{$\begin{array}{l}\text { REPORTED BY VEN- } \\
\text { DOR FROM PRESSURE } \\
\text { VESSEL FLANGE AREA }\end{array}$} & 29,000 & 79,200 & 53 & 66 \\
\hline & 29,600 & 79,700 & 53 & 65 \\
\hline \multicolumn{5}{|c|}{ SPECIMENS PULLED AT AGC WHICH WERE ANNEALED WITH JACKET FROM P.V. FLANGE AREA } \\
\hline 5 B & 30,600 & 80,200 & 55.4 & 65.6 \\
\hline $6 \mathrm{~B}$ & 31,100 & 79,200 & 58.9 & 65.9 \\
\hline 7 B & 29,300 & 78,800 & 61.4 & 62.4 \\
\hline 8 B & 28,200 & 78,500 & 60.0 & 64.4 \\
\hline \multicolumn{5}{|c|}{ FROM EXIT END OF NOZZLE } \\
\hline $1 \mathrm{~A}$ & 34,800 & 83,400 & 52.1 & 59.7 \\
\hline THRU & TO & TO & TO & TO \\
\hline $4 \mathrm{~A}$ & 38,400 & 84,100 & 57.1 & 65.4 \\
\hline
\end{tabular}

RN-S-0338 STATES THAT "THE HYDROTEST CONDITION, EXCEPT IN THE CASE OF THE FORWARD CLOSURE BOLTS, WAS FOUND TO BE CRITICAL IN EACH CASE." THE MAXIMUM STRESS INDUCED IN THE JACKET IS IN THE HYDROTEST AND IS 21,400 PSI. 


\begin{tabular}{|l|c|c|c|}
\cline { 2 - 4 } \multicolumn{1}{c|}{} & SMALL & MEDIUM & LARGE \\
\hline FORWARD OF THROAT & 2 & 1 & 2 \\
\hline THROAT & 16 & & \\
\hline AFT OF THROAT & 9 & 3 & 3 \\
\hline
\end{tabular}

SMALL $\quad .125$ LONG $\times .030$ DEEP (MAXIMUM)

MEDIUM .125 LONG TO.250 LONG $\times \quad .030$ TO .080 DEEP

LARGE $\quad .250$ LONG TO .500 LONG $\times \quad .080$ TO .125 DEEP

BLUEPRINT CRITERIA:

8 INCHES EITHER SIDE OF THROAT - .03 DEEP WITH .06 MIN. RADIUS OTHER THAN THROAT AREA - .12 WITH .06 MIN. RADIUS 


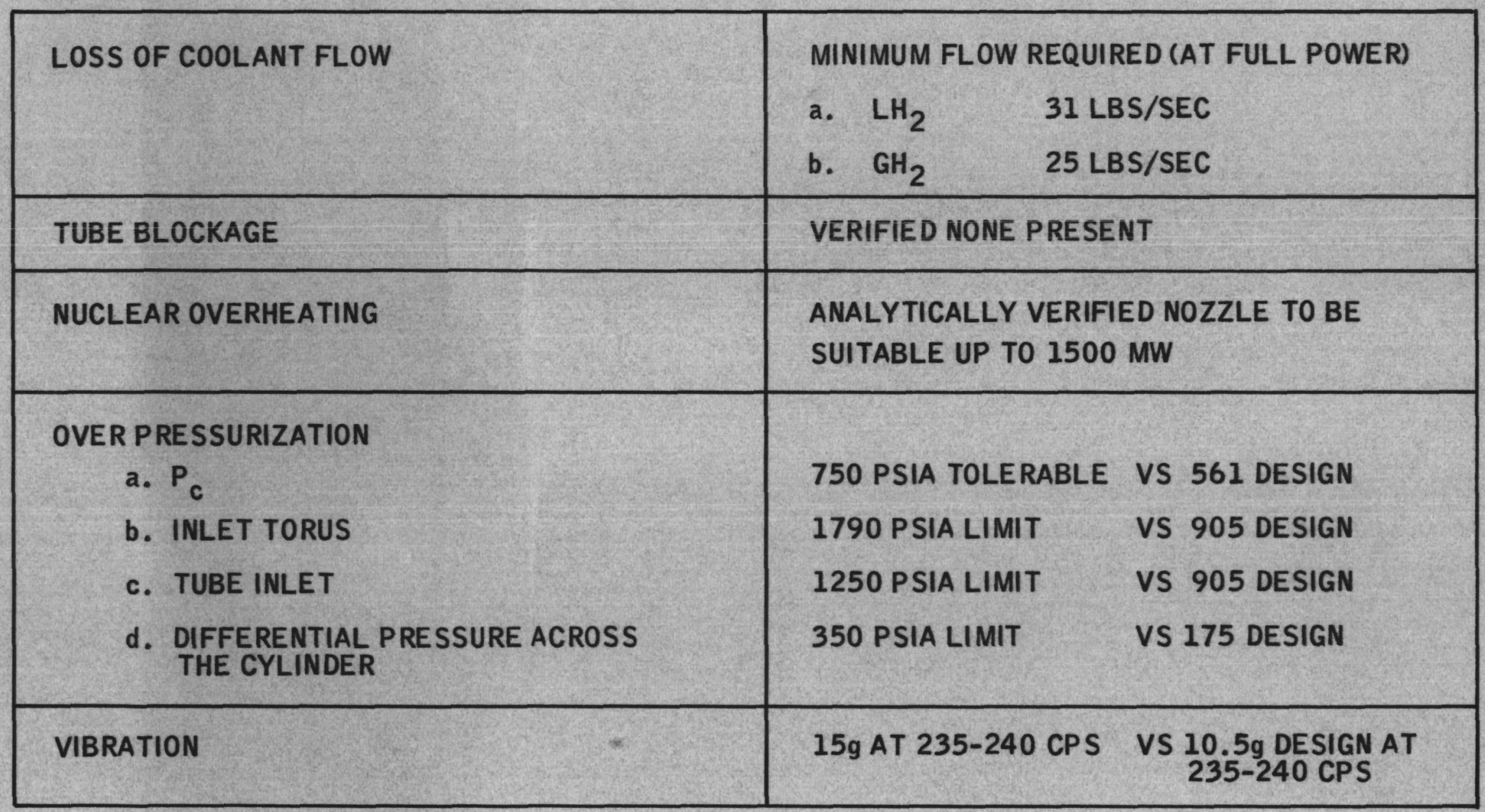




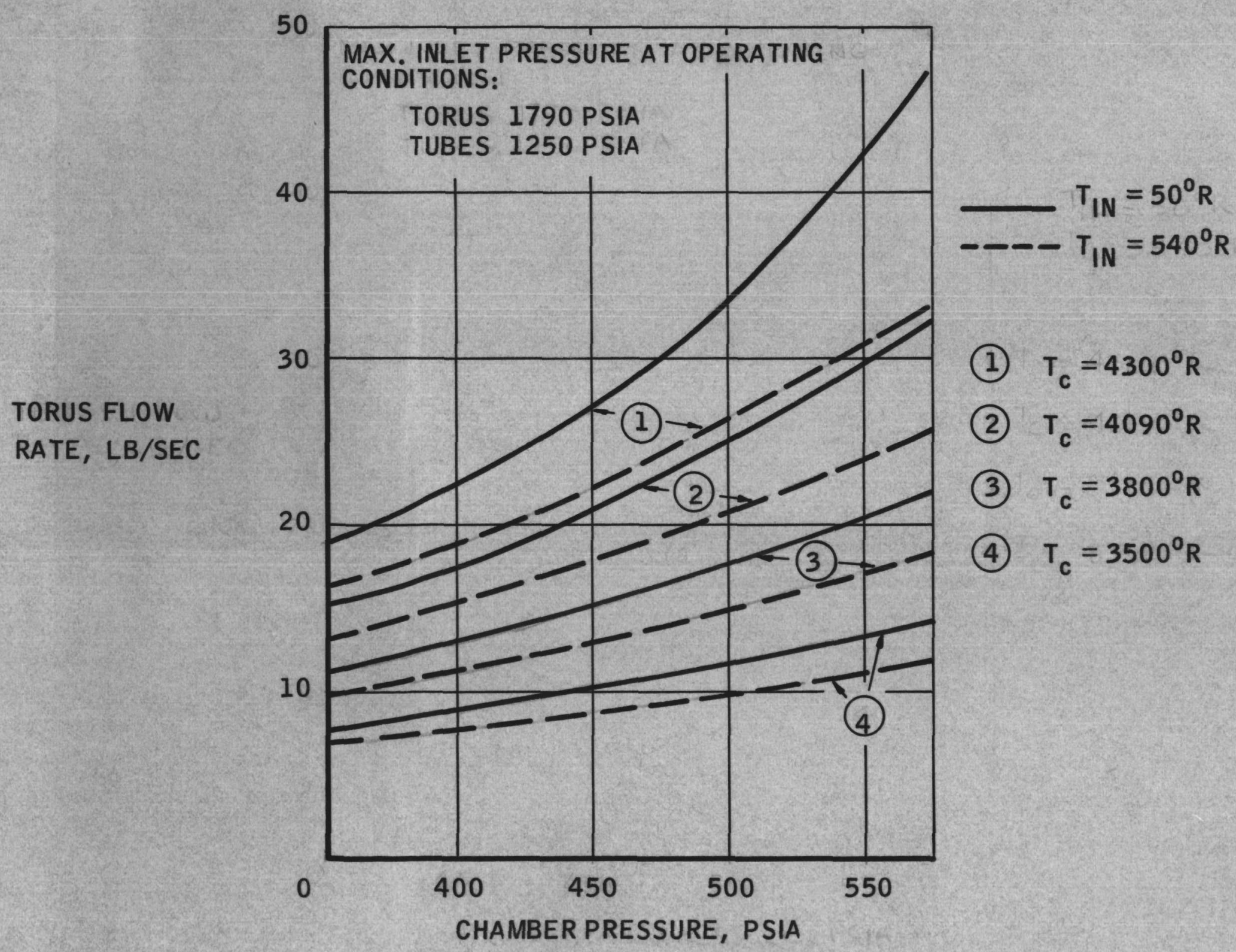




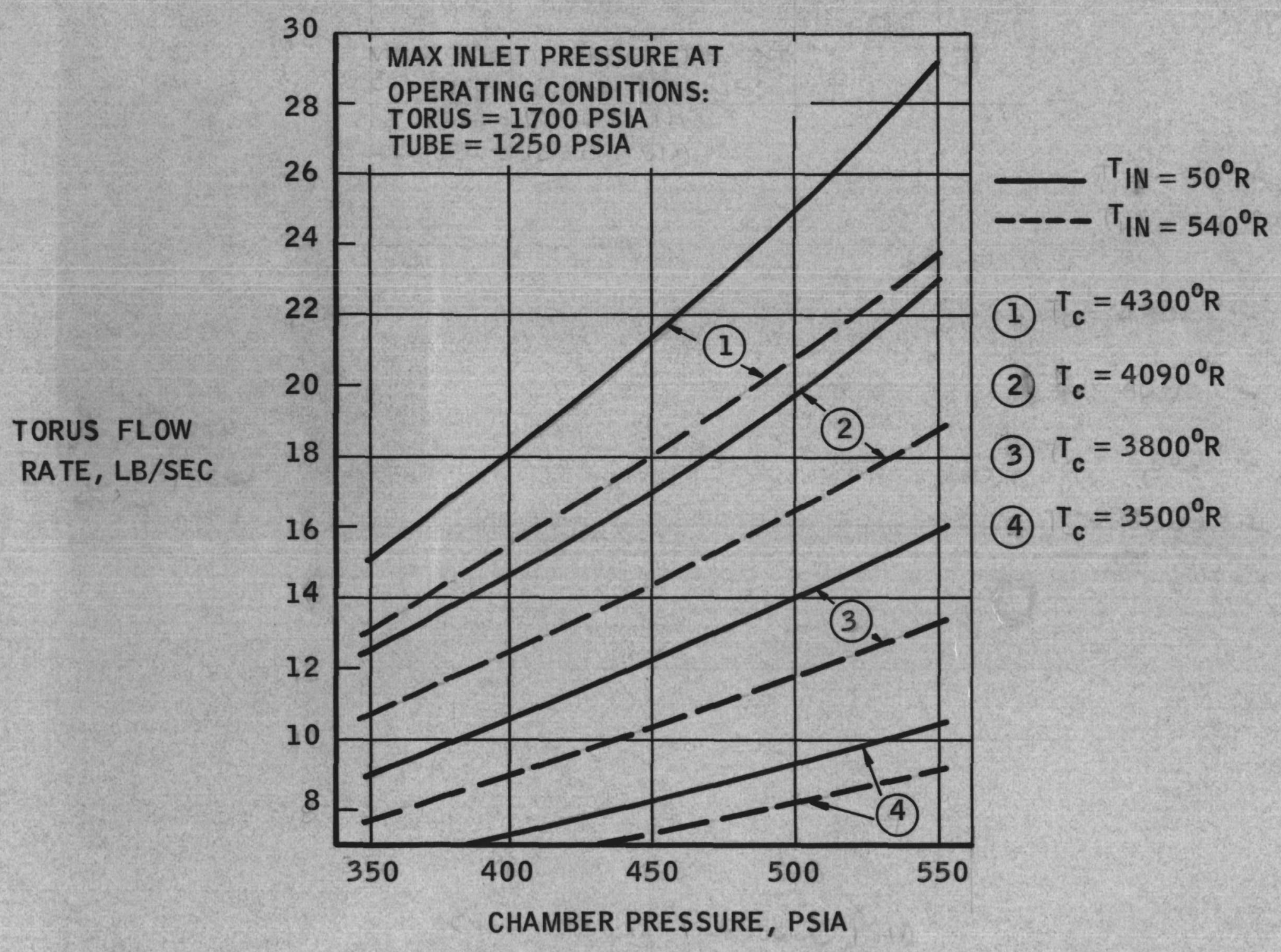


BLEED PORT BOSS - TWO CONCENTRIC BORES CALLED OUT TO BE CONCENTRIC WITHIN .001 ARE WITHIN .005. (I.R. 412453)

5 OF 6 INSTRUMENTATION BOSSES ON TORUS ARE MISLOCATED. (I.R. 417199)

RADIOGRAPHIC INSPECTION SHOWED FOREIGN MATERIAL, 2 INCHES LONG $x .045$ WIDE IN ONE TUBE AND .100 LONG PROTRUSION IN ANOTHER. (I.R. 420720)

POROSITY IN WELD OF AFT FLANGE TO NOZZLE. (I.R. 420720)

SCRATCHES ON O D OF PRESSURE VESSEL FLANGE

DENTS IN TUBES 58 \& 176 WITH RADIUS LESS THAN .06.

YIELD STRENGTH OF JACKET IS AS LOW AS 28200 PSI. 


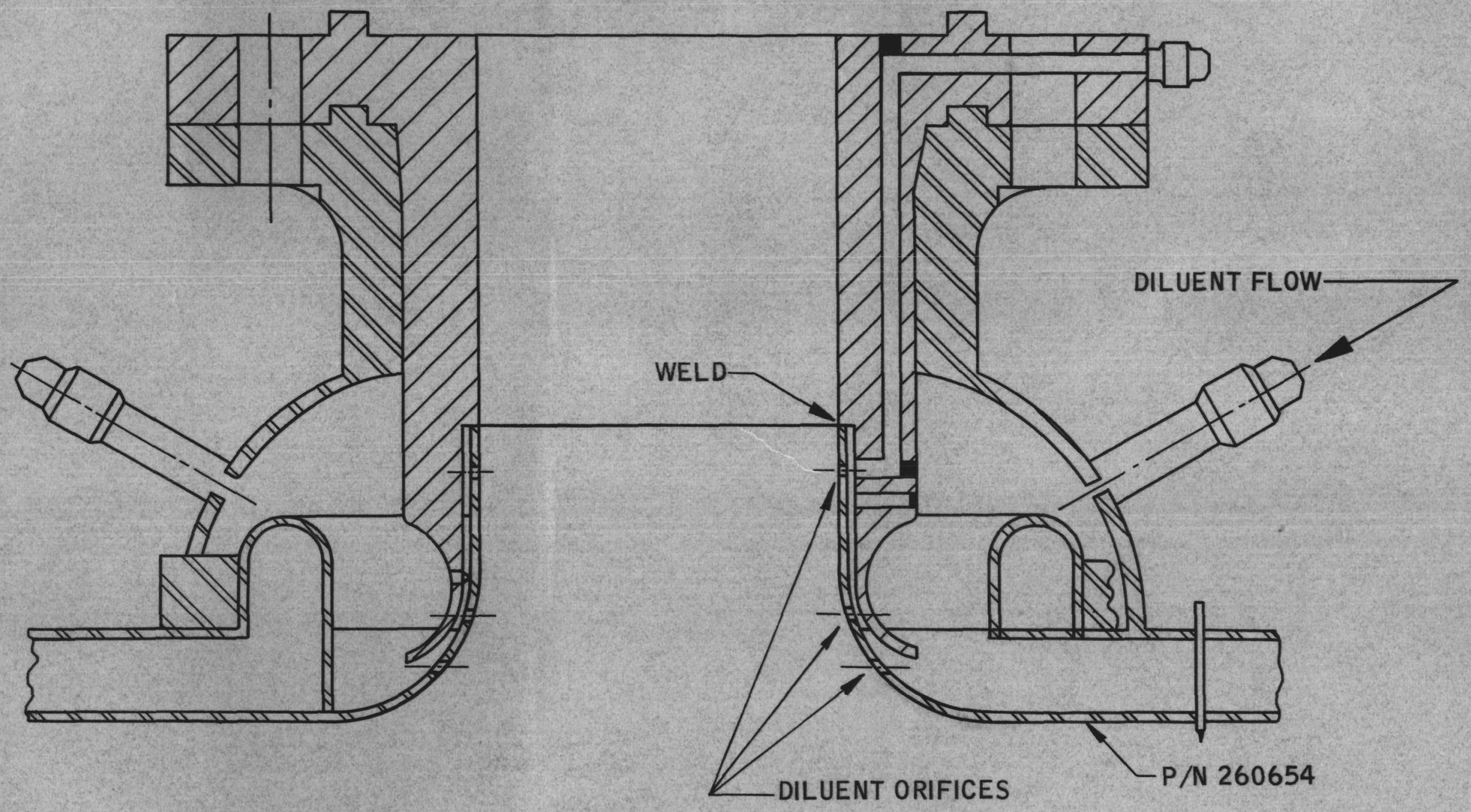



$Q_{\text {NERVA }}=Q_{\text {NRX-A6 }}\left(\frac{\text { POWER }_{\text {NERVA }}}{\text { POWER }_{\text {NRX-A6 }}}\right)\left(\frac{\text { DENSITY HASTELLOY X }_{\text {HAST }}}{\text { DENSITY CRES } 347^{2}}\right) 1.5$

1. FACTOR OF 1.5 REFLECTS UNCERTAINTY BASED ON HISTORICAL DIFFERENCES BETWEEN PREDICTED AND TEST DATA VALUES

2. $Q=B T U / N^{3}-S E C$ 







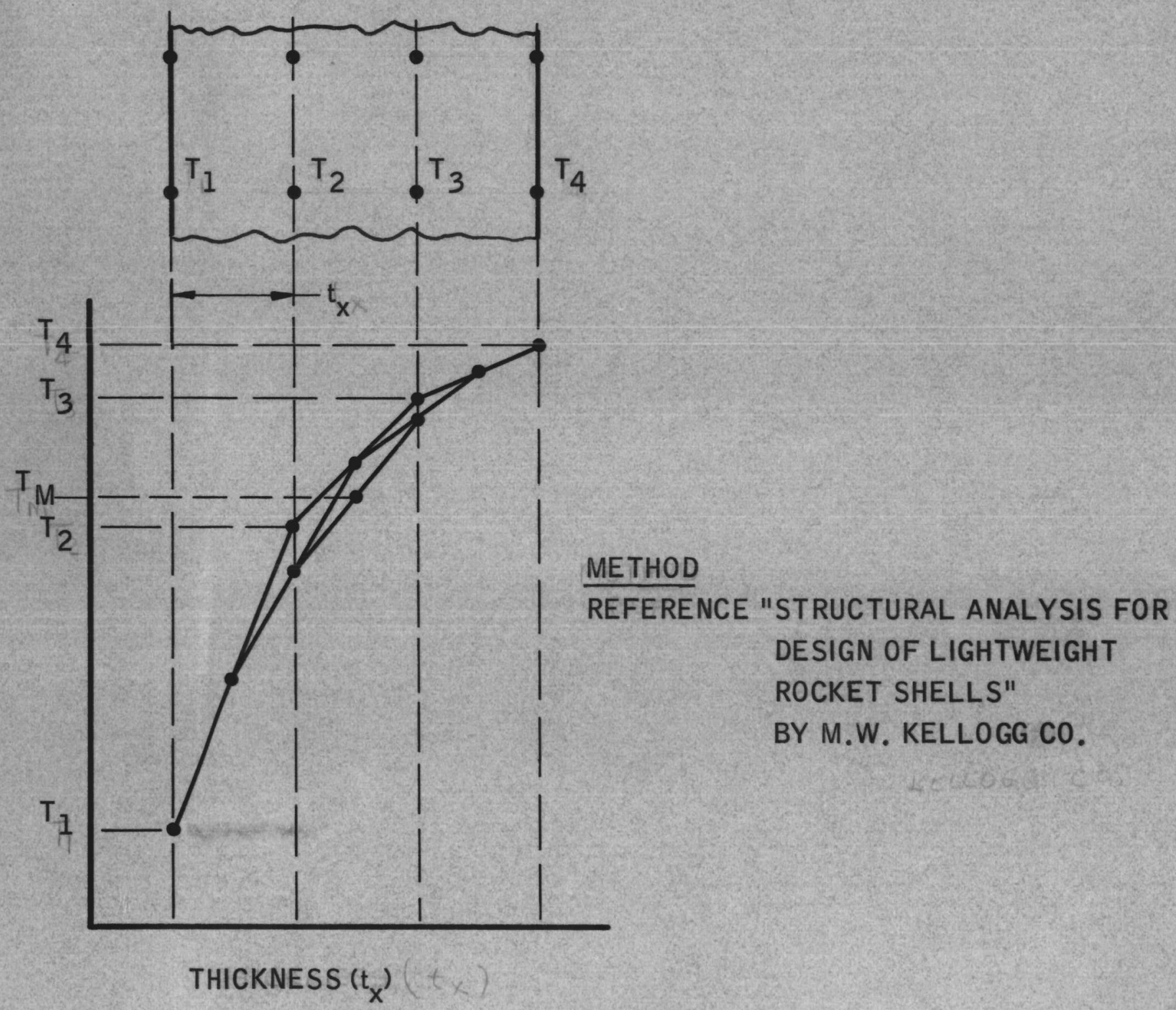


MEAN WALL TEMPERATURES AT MAXIMUM JACKET THICKNESS

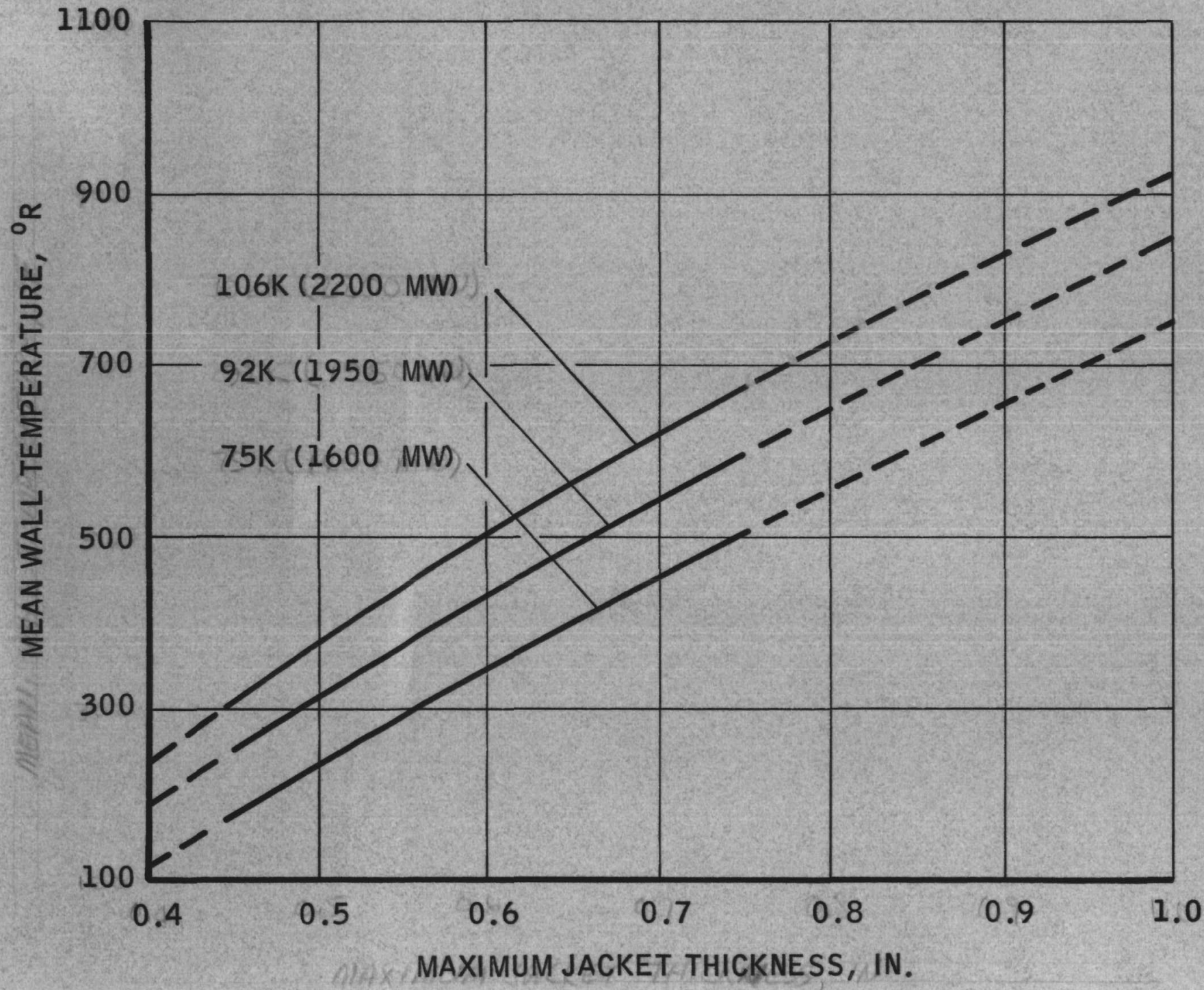


MEAN WALL TEMPERATURES AT MAXIMUM JACKET THICKNESS

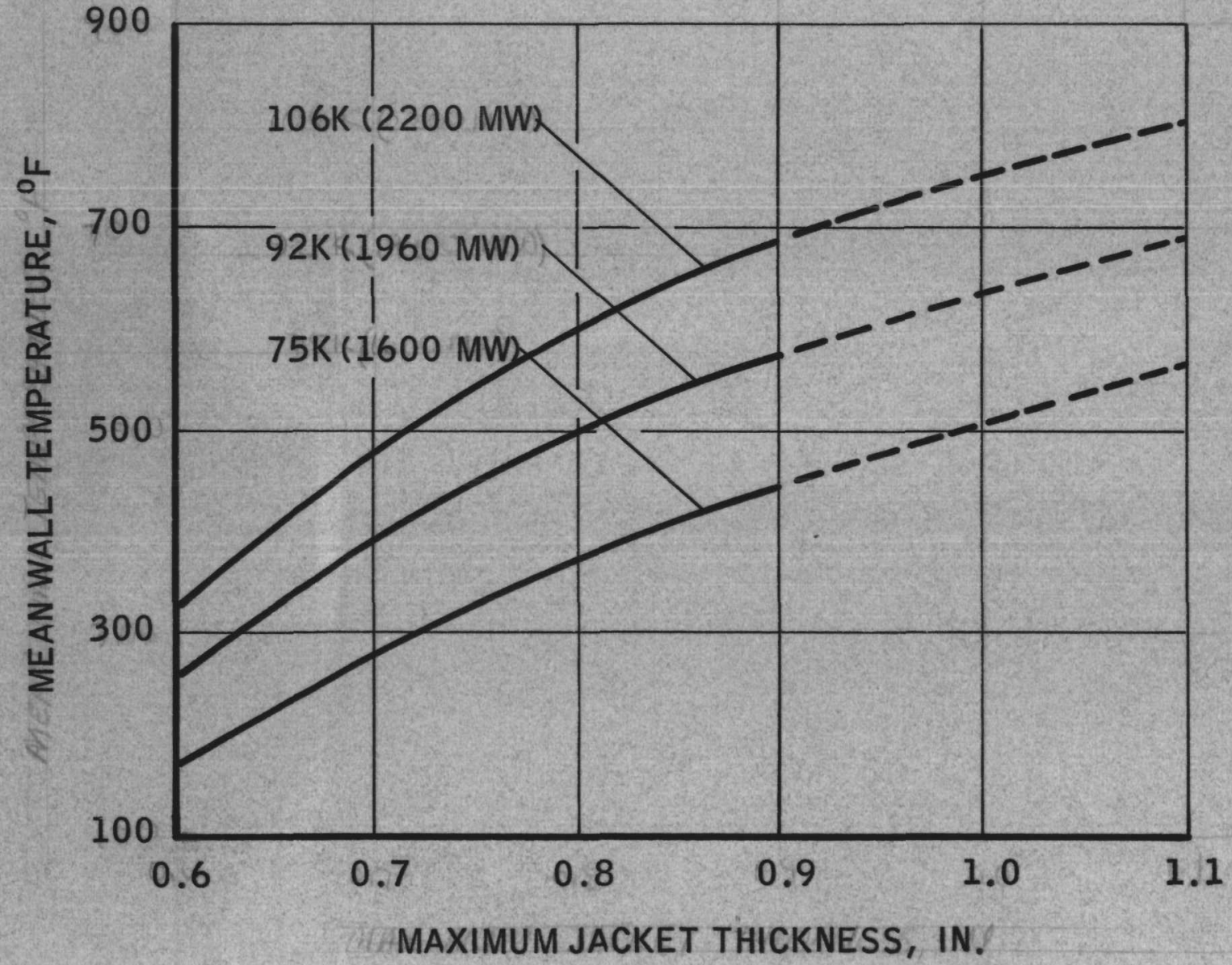




\section{NUCLEAR - THERMAL}

ROM RELATIONSHIP

$$
\begin{aligned}
\Delta T_{2}=\Delta T_{1}\left(\frac{t_{2}}{t_{1}}\right)^{2}\left(\frac{M W_{2}}{M W_{1}}\right)\left(\frac{k_{n_{2}}}{k_{n_{1}}}\right)\left(\frac{P_{2}}{P_{1}}\right) & \text { (ASSUMES } k \text { IS CONSTANT) } \\
\text { AND } \frac{M W_{1}}{M W_{2}} \ltimes \frac{P_{c_{1}}}{P_{c_{2}}} \propto \frac{F_{1}}{F_{2}} & \begin{array}{l}
\text { IF } A_{t} \text { IS CONSTANT } \\
\text { W } \propto P_{c}
\end{array}
\end{aligned}
$$

\section{EXACT ANALYSIS PER COMPUTER PROGRAM}

STRESS

$$
\begin{aligned}
\sigma_{\text {HOOP }} & =\frac{P_{C} R_{M E A N}}{t \cos \theta}(1.10)^{*}\left(\frac{t}{t-.050}\right) \\
\text { M.S. } & =\frac{.85 F_{\text {ty }}}{\sigma_{\text {HOOP }}}-1
\end{aligned}
$$

* $1.10=$ FACTOR TO INCLUDE THE INCREASE IN HOOP MEMBRANE STRESS AS A RESULT OF THE DISCONTINUITY AT THE INTERFACE 


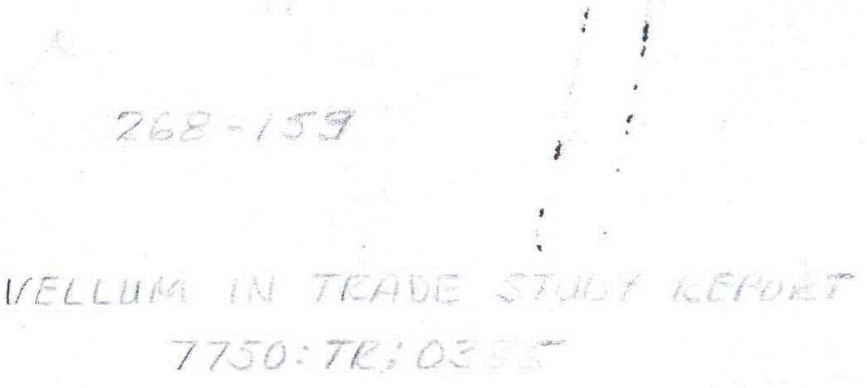




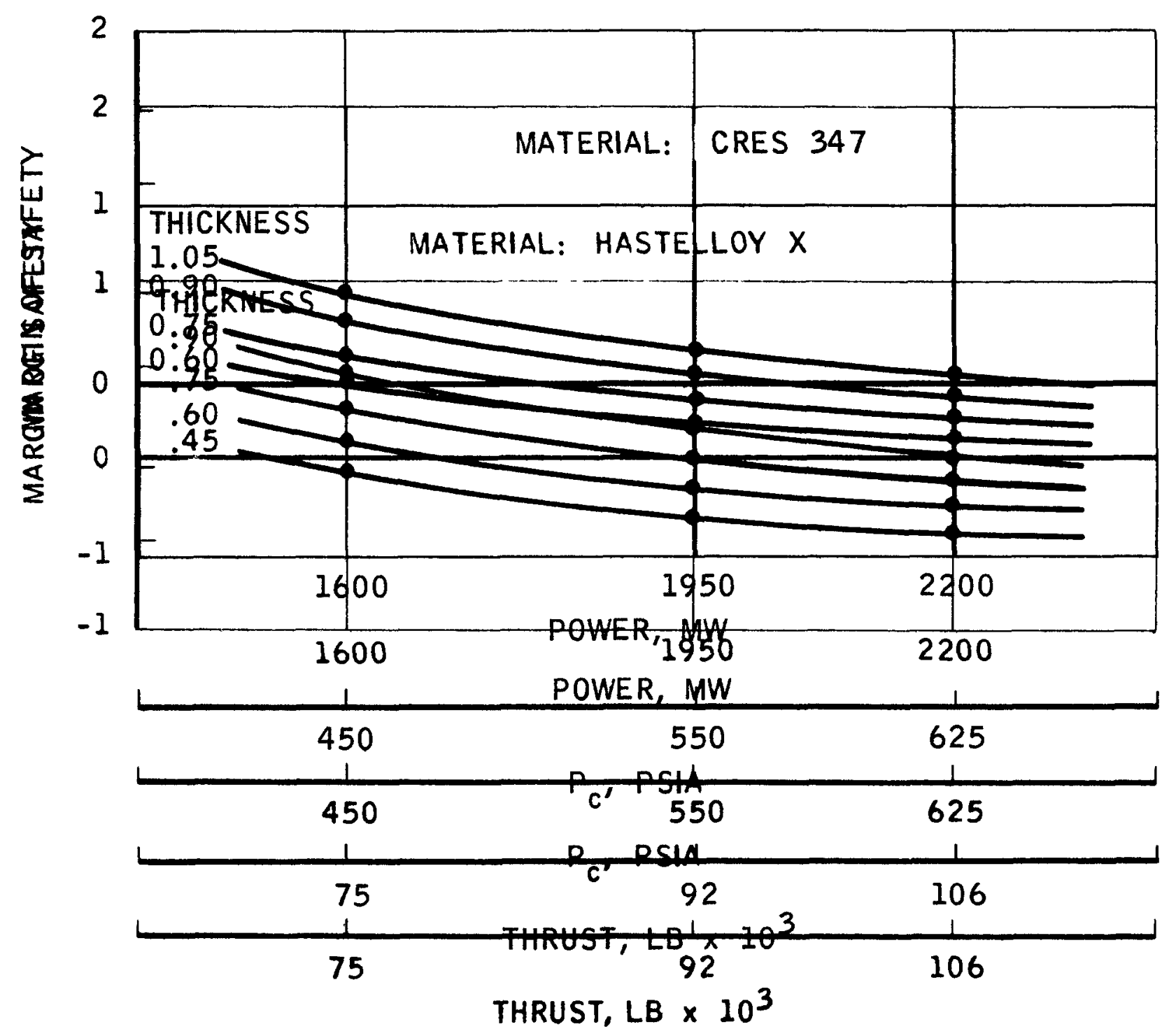




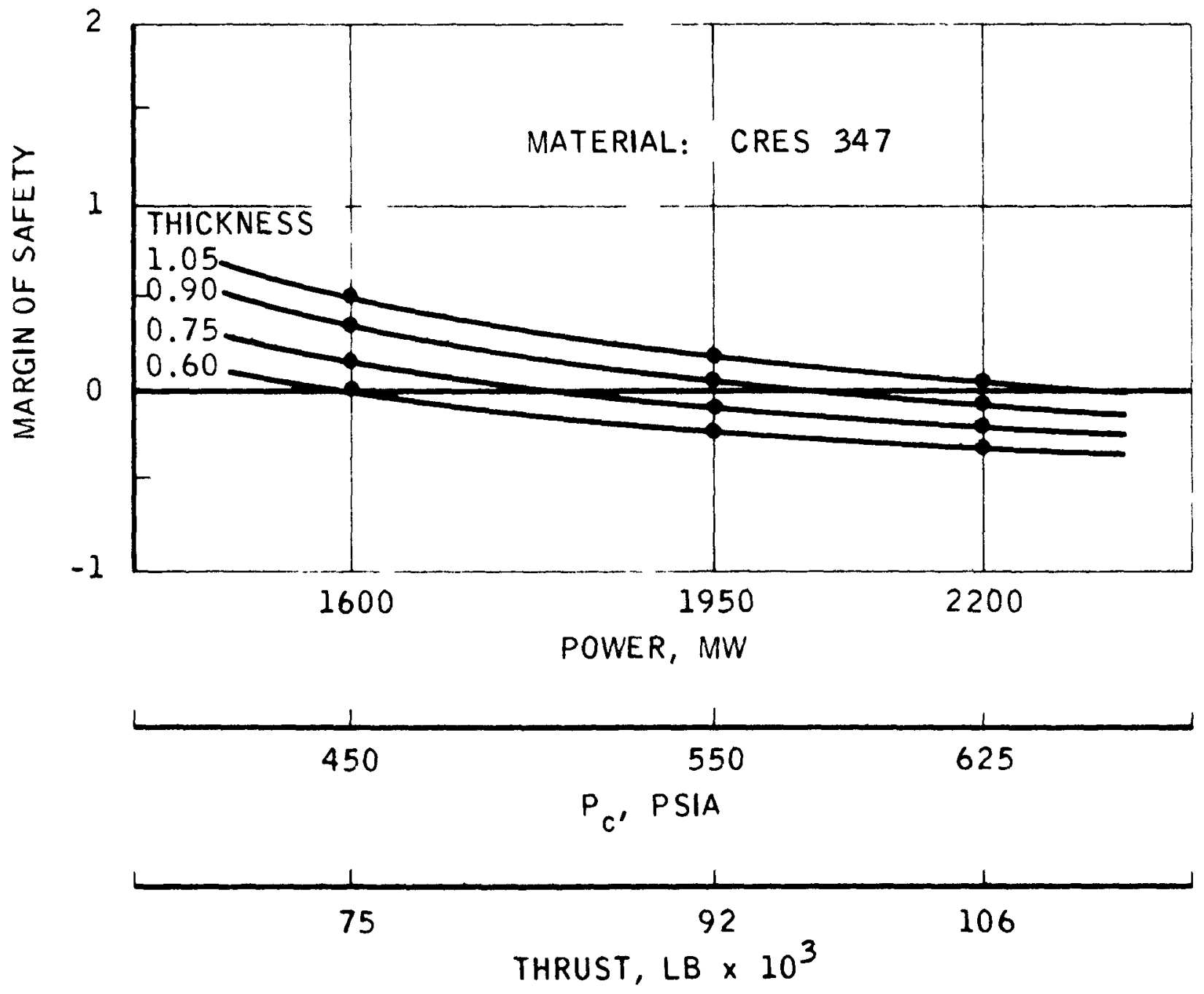




$$
\begin{aligned}
& P_{347}=.290 \mathrm{LB} / \mathrm{IN}^{3} \\
& P_{\text {HAST } x}=.297 \mathrm{LB} / \mathrm{IN}^{3}
\end{aligned}
$$

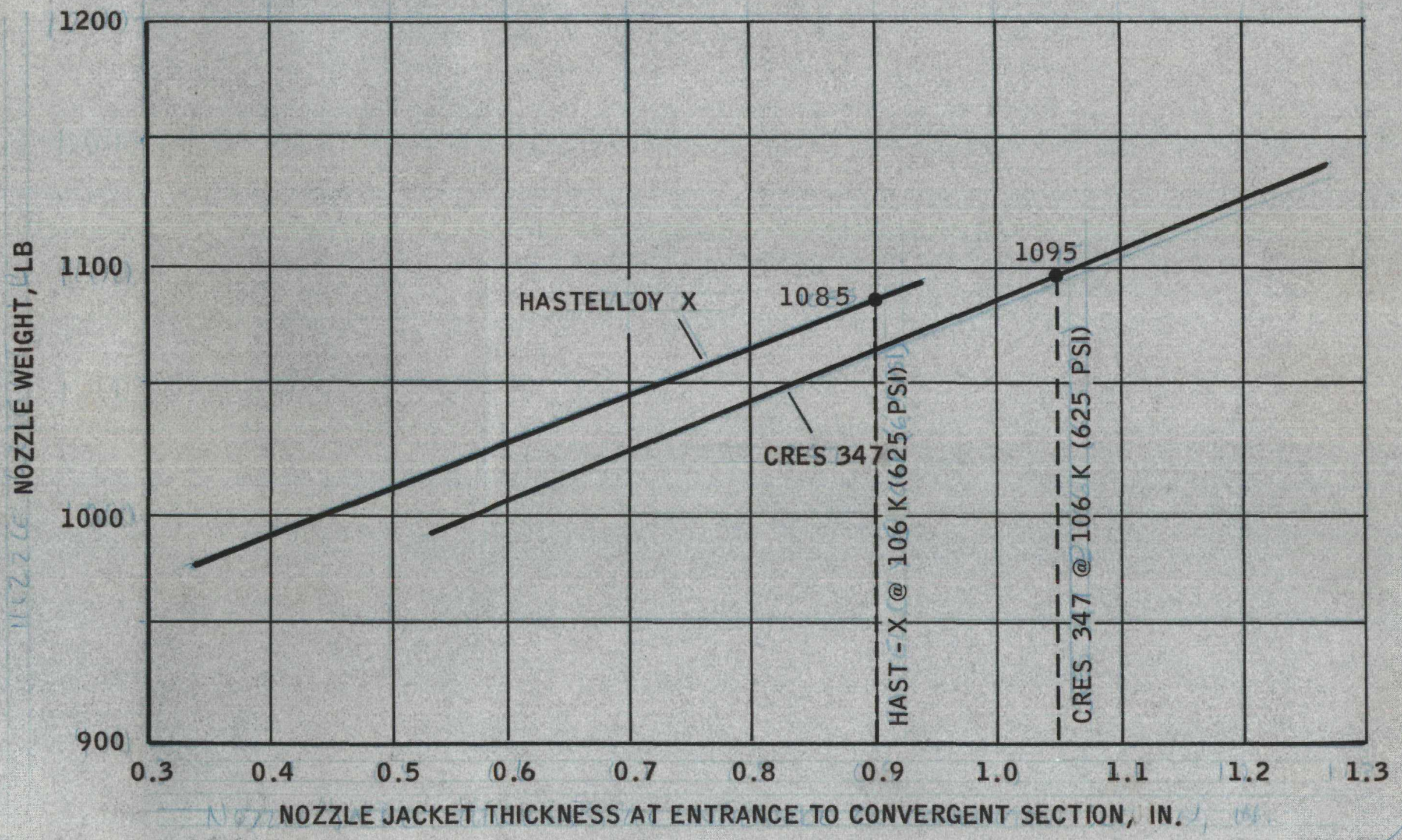




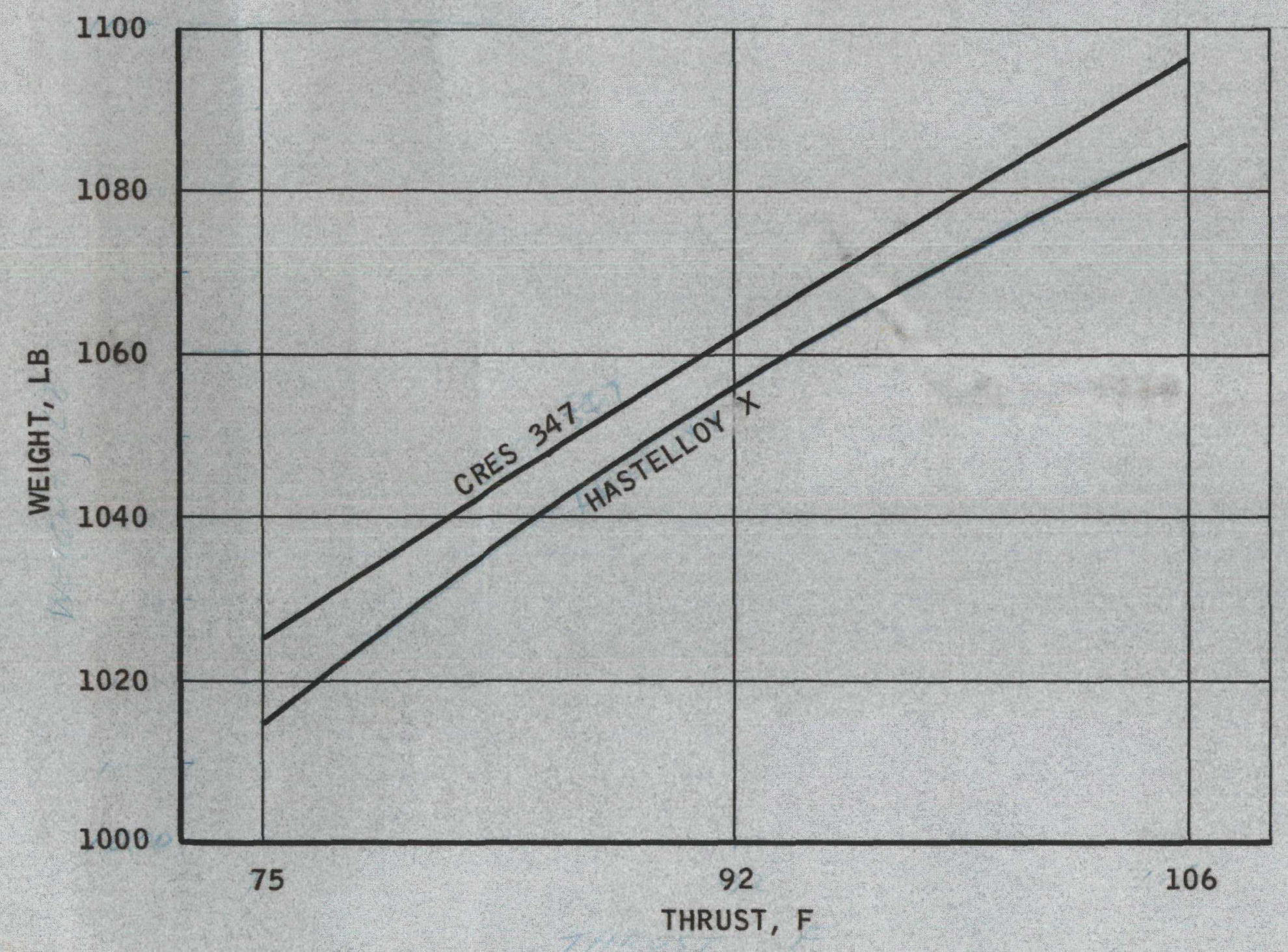




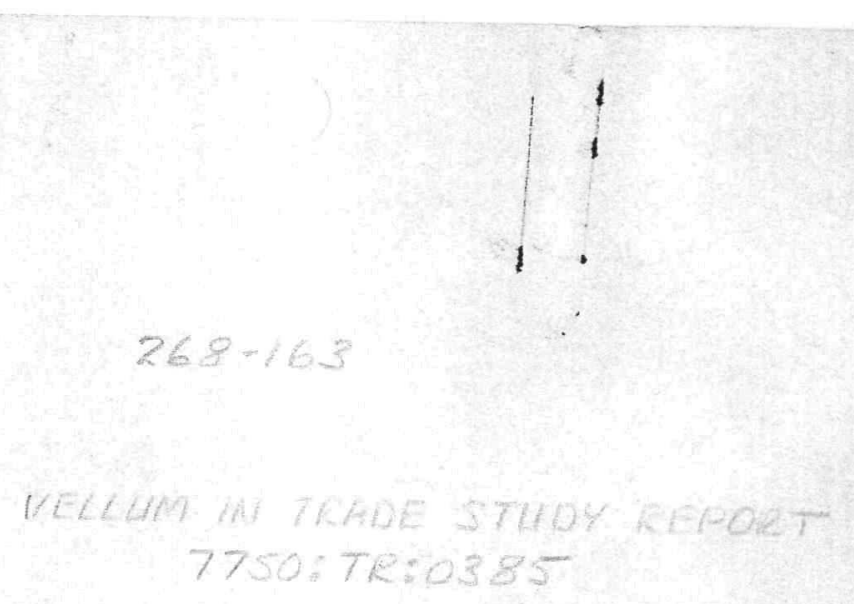




\begin{tabular}{|c|c|c|c|c|c|c|c|c|}
\hline \multirow[b]{2}{*}{ LOCATION } & \multirow[b]{2}{*}{$\triangle P, P S I$} & \multirow{2}{*}{$\begin{array}{c}\text { MAX } \\
{ }^{T} W^{\prime}{ }^{\circ} F\end{array}$} & \multirow{2}{*}{$\begin{array}{c}\text { MEAN } \\
\text { TWM' }^{\circ}{ }^{\circ} \mathrm{F}\end{array}$} & \multirow[b]{2}{*}{$\mathrm{Rg}$} & \multicolumn{2}{|c|}{ WALL THICKNESS } & \multicolumn{2}{|c|}{ MARGIN OF SAFETY } \\
\hline & & & & & 347 & HAST-X & 347 & HAST-X \\
\hline BARREL & 230 & 1300 & 1140 & .281 & .014 & .012 & 2.33 & 3.29 \\
\hline THROAT & 475 & 1600 & 1190 & .075 & .014 & .012 & 5.16 & 7.10 \\
\hline $4: 1$ & 890 & 800 & 710 & .156 & .014 & .012 & .83 & 1.41 \\
\hline $7: 1$ & 910 & 700 & 640 & .208 & .014 & .012 & .30 & .80 \\
\hline $\begin{array}{l}347 \\
\text { JACKET }\end{array}$ & 450 & 505 & 270 & 18.638 & .750 & - & .20 & - \\
\hline $\begin{array}{l}\text { HAST-X } \\
\text { JACKET }\end{array}$ & 450 & 500 & 290 & 18.568 & - & .600 & - & .10 \\
\hline
\end{tabular}




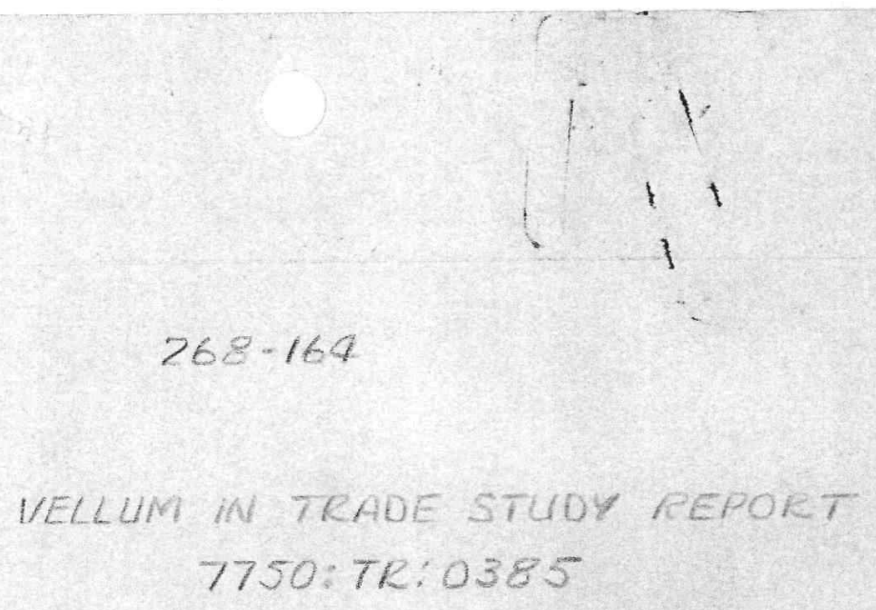


PRELIMINARY $75 \mathrm{~K}$ NOZZLE

TUBE DESIGN PARAMETERS

(HYDROTEST CONDITIONS)

\begin{tabular}{|c|c|c|c|c|c|}
\hline \multirow[b]{2}{*}{ MATERIAL } & \multirow[b]{2}{*}{ LOCATION } & \multicolumn{2}{|c|}{ OPERATING } & \multicolumn{2}{|c|}{ HYDROTEST } \\
\hline & & $\triangle P$ & MS & $\triangle P$ & $M S^{*}$ \\
\hline \multirow[t]{4}{*}{347} & BARREL & 230 & 2.33 & 405 & .05 \\
\hline & THROAT & 475 & 5.16 & 885 & 2.84 \\
\hline & $4: 1$ & 890 & .83 & 1350 & .75 \\
\hline & $7: 1$ & 910 & .30 & $1410 *$ & .30 \\
\hline \multirow[t]{4}{*}{ HAST-X } & BARREL & 230 & 3.29 & 400 & .31 \\
\hline & THROAT & 475 & 7.10 & 840 & 4.24 \\
\hline & $4: 1$ & 890 & 1.41 & $1300 *$ & 1.41 \\
\hline & $7: 1$ & 910 & .80 & 300 & .80 \\
\hline
\end{tabular}

*HYDROTEST PRESSURE IS BASED ON THE MAXIMUM EXPECTED OPERATING PRESSURE IN THE COOLING CIRCUIT, WITH A TEMPERATURE COMPENSATING FACTOR APPLIED.

A ONE ZONE PRESSURE CHECK IS ASSUMED, IF THE NOZZLE WERE UPGRADED TO $106 \mathrm{~K}$, A TWO ZONE PROOF TEST WOULD PROBABLY BE REQUIRED. 


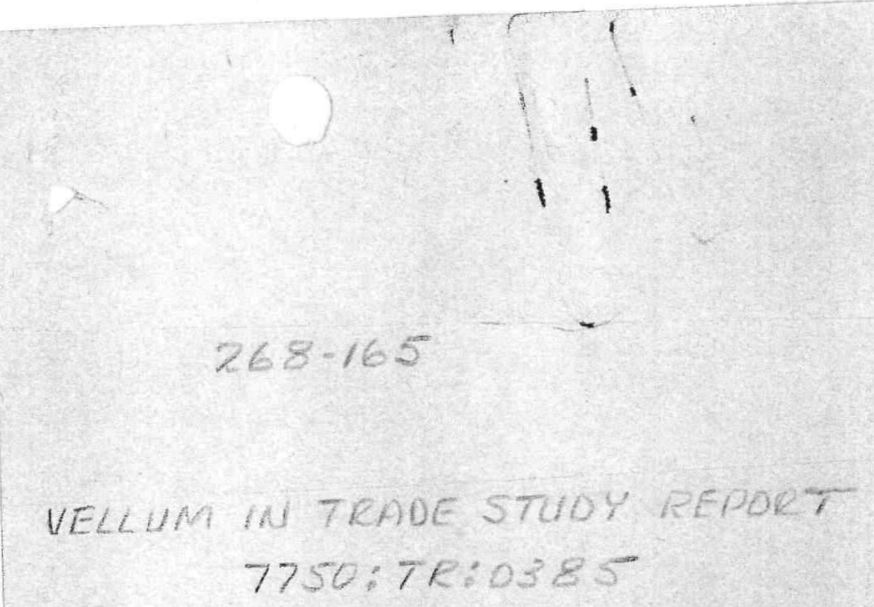




\begin{tabular}{|c|c|c|c|c|}
\hline & $\begin{array}{l}\text { TECHNOLOGY } \\
\text { NOZZLE, S/N } 021 \\
\text { (M.O. INJ.) }\end{array}$ & $\begin{array}{l}\text { TECHNOLOGY } \\
\text { NOZZLE, S/N } 027 \\
\text { (M.O. INJ.) }\end{array}$ & $\begin{array}{c}\text { PHOEBUS } \\
\text { NOZZLE，S/N } 601 \\
\text { (M.O. INJ.) }\end{array}$ & $\begin{array}{c}\text { PHOEBUS } \\
\text { NOZZLE, S/N } 602 \\
\text { (COAX. INJ.) }\end{array}$ \\
\hline $\begin{array}{l}\text { NUMBER OF } \\
\text { TESTS }\end{array}$ & 10 & 10 & 3 & 13 \\
\hline $\begin{array}{l}P_{C} \text { RANGE } \\
\text { (PSIA) }\end{array}$ & $605-652$ & $437-801$ & $674-705$ & $590 \star-686$ \\
\hline MR RANGE & $4.9-8.3$ & $1.5 *-5.8$ & $4.9-5.4$ & $5.3-5.9$ \\
\hline $\begin{array}{l}\text { TUBE WALL } \\
\text { TEMP RANGE, }{ }^{\circ} \mathrm{F}\end{array}$ & $2015-2275$ & $2000-2100$ & $2000-2100$ & $2000-2100$ \\
\hline $\begin{array}{l}\text { COOLANT FLOW } \\
\text { RANGE, LB/SEC }\end{array}$ & $62.9-73.5$ & $43.2-77.1$ & $235-239$ & $205-242$ \\
\hline REMARKS & $\begin{array}{l}\text { ONE TUBE ERODED } \\
\text { THRU ON TEST } 2 \\
\text { DUE TO OBSTRUCT- } \\
\text { ION BY A PIECE OF } \\
\text { KEL F LIP SEAL. } \\
\text { NO ADD'L DAMAGE } \\
\text { ON SUBSEQUENT } \\
\text { TESTS. }\end{array}$ & $\begin{array}{l}3 \text { TUBES ERODED } \\
\text { THRU ON TEST } 10 \\
\text { AT NOZZLE } \\
\text { COOLANT OF } \\
43.2 \mathrm{LB} / \mathrm{SEC}\end{array}$ & $\begin{array}{l}\text { NUMEROUS } \\
\text { TRANSVERSE } \\
\text { CRACKS IN } 10 \\
\text { TUBES AFTER } \\
\text { TEST } 3\end{array}$ & $\begin{array}{l}\text { ONE SMALL } \\
\text { TRANSVERSE } \\
\text { CRACK IN ONE } \\
\text { TUBE UPSTREAM } \\
\text { OF THROAT - } \\
\text { TEST } 13\end{array}$ \\
\hline
\end{tabular}

* INCLUdes EMERGENCY SHUTdOWN DATA WHERE STEAdY STATE CONDITIONS WERE NOT ACHIEVED 


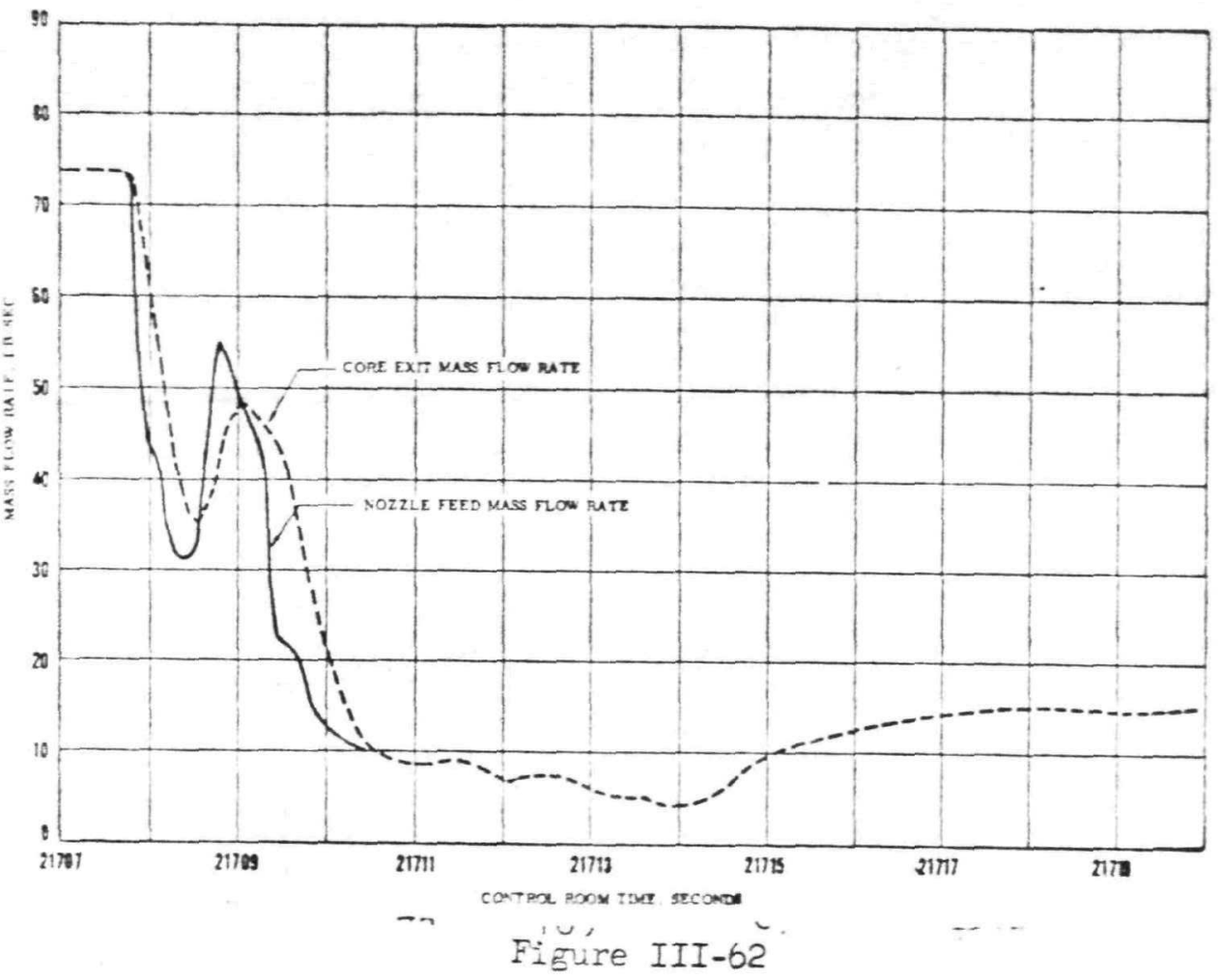



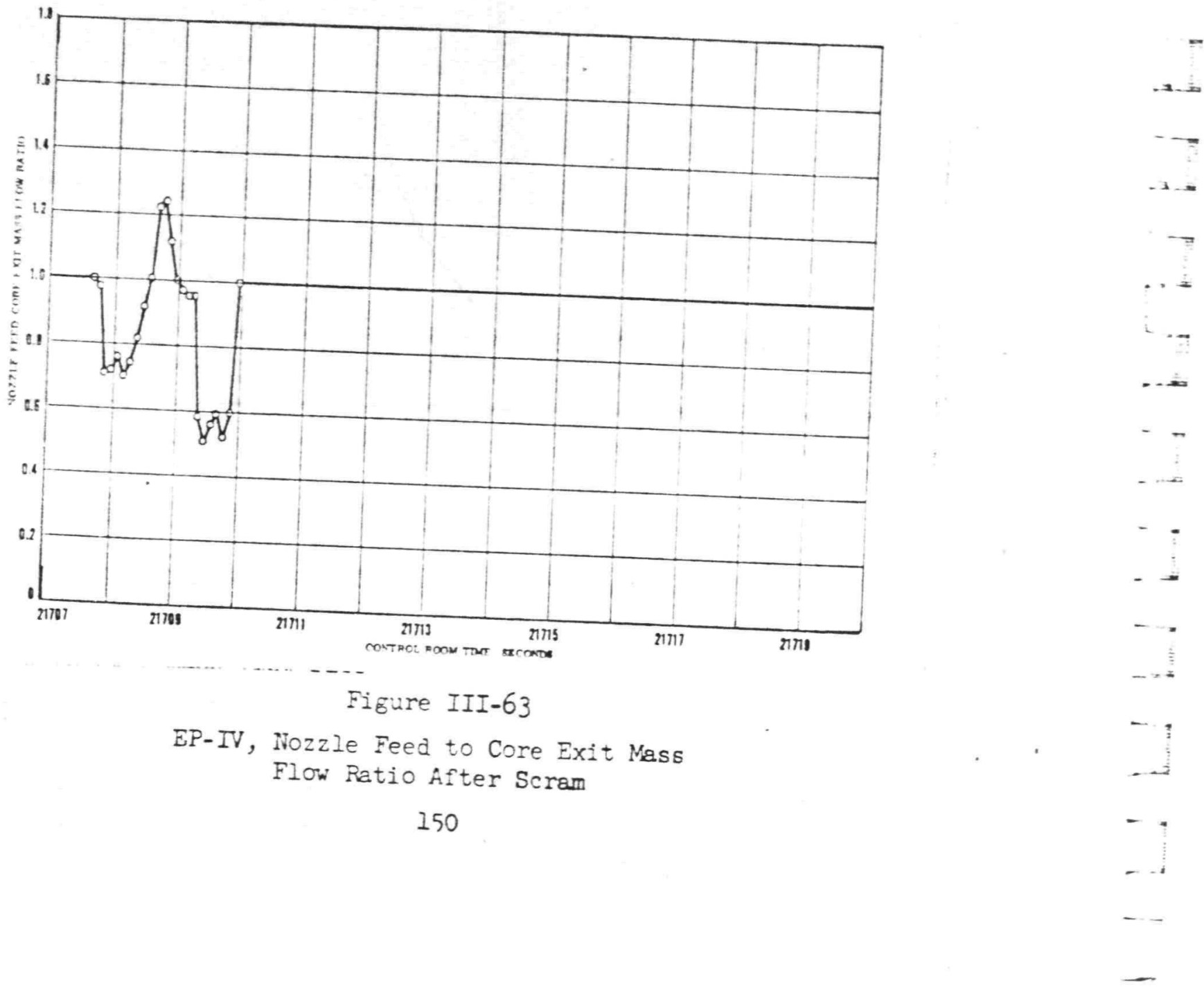


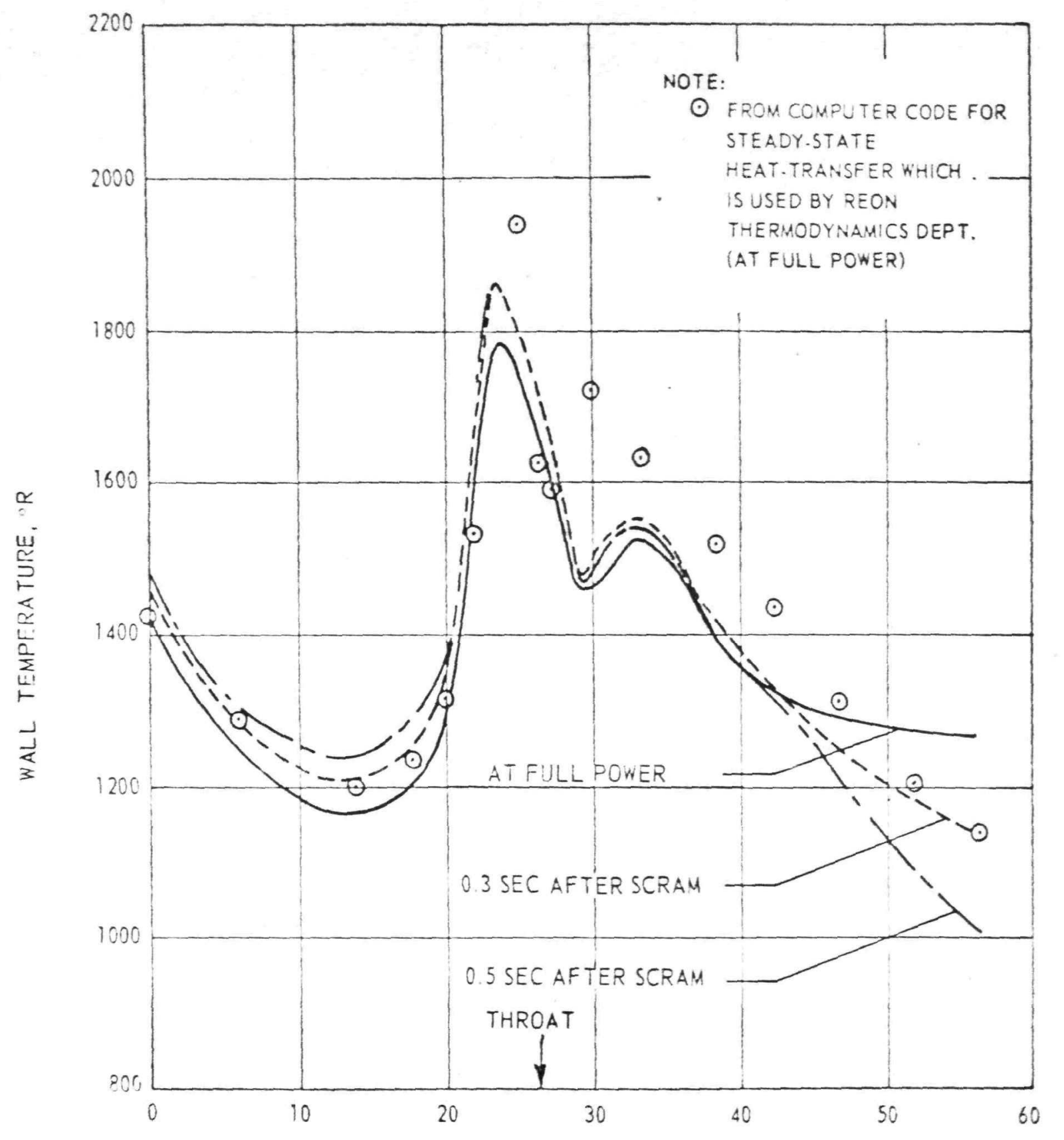

AXIAL DISTANCE FROM CORE EXIT, IN. 

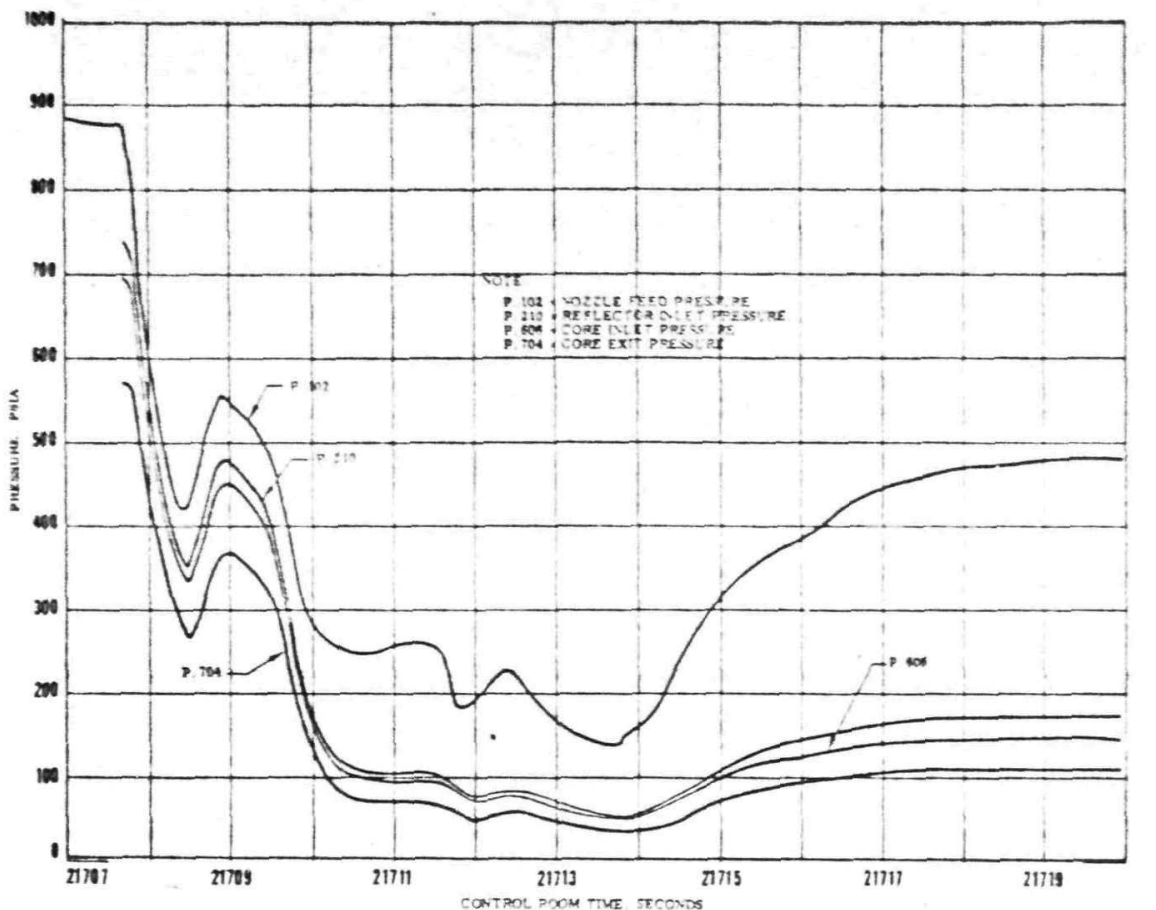

Figure III-54

EP-IV, Fluid Pressure Characteristics in Nozzle and Reactor After Scram

- 


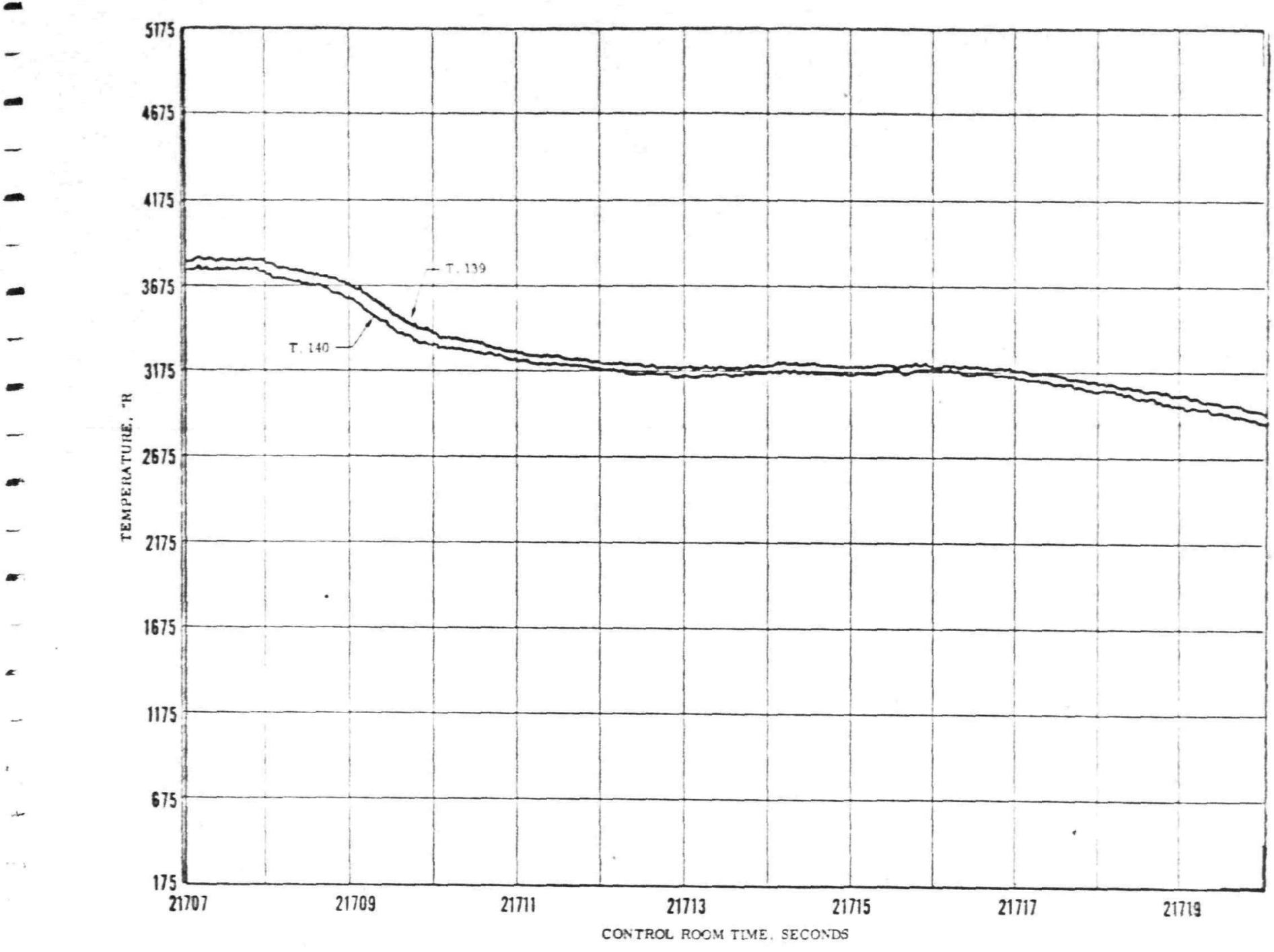




\begin{tabular}{|c|c|c|c|}
\hline S/N & BRAZE CYCLES & $\begin{array}{c}\text { FIRST LEAK TEST } \\
\text { 1450 PSIG }\end{array}$ & $\begin{array}{c}\text { FINAL LEAK TEST } \\
50 \text { PSIG He }\end{array}$ \\
\hline 880600 & 4 & 552 & 0 \\
\hline 880601 & 3 & 373 & 0 \\
\hline 880603 & 3 & 103 & 0 \\
\hline 880604 & 3 & 171 & 0 \\
\hline 880605 & 3 & 119 & 0 \\
\hline
\end{tabular}




\begin{tabular}{|l|l|l|l|l|l|l|l|l|l|l|l|l|l|}
\hline \multicolumn{1}{|c|}{ S/N } & 008 & 009 & 021 & 022 & $023 \times$ & 024 & 025 & 026 & 027 & 028 & 029 & 030 & 031 \\
\hline $\begin{array}{l}\text { NO. LEAKS } \\
\text { AS BRAZED }\end{array}$ & $\begin{array}{l}\text { APPROX } \\
600\end{array}$ & 429 & MANY & 50 & 11 & 8 & 8 & 5 & 15 & 6 & 193 & 3 & 11 \\
\hline $\begin{array}{l}\text { NO. LEAKS } \\
\text { AS REPAIRED }\end{array}$ & 0 & 0 & 0 & 0 & 4 & 0 & 0 & 0 & 0 & 0 & 0 & 0 & 0 \\
\hline
\end{tabular}

*CFDTS AND XECF NOZZLE 4 LEAKS BLEED PORT END CAPS 
THERMAL CYCLE DATA FOR HASTELLOY $X$ \& CRES 347 THIN-WALL

TUBE BUNDLES

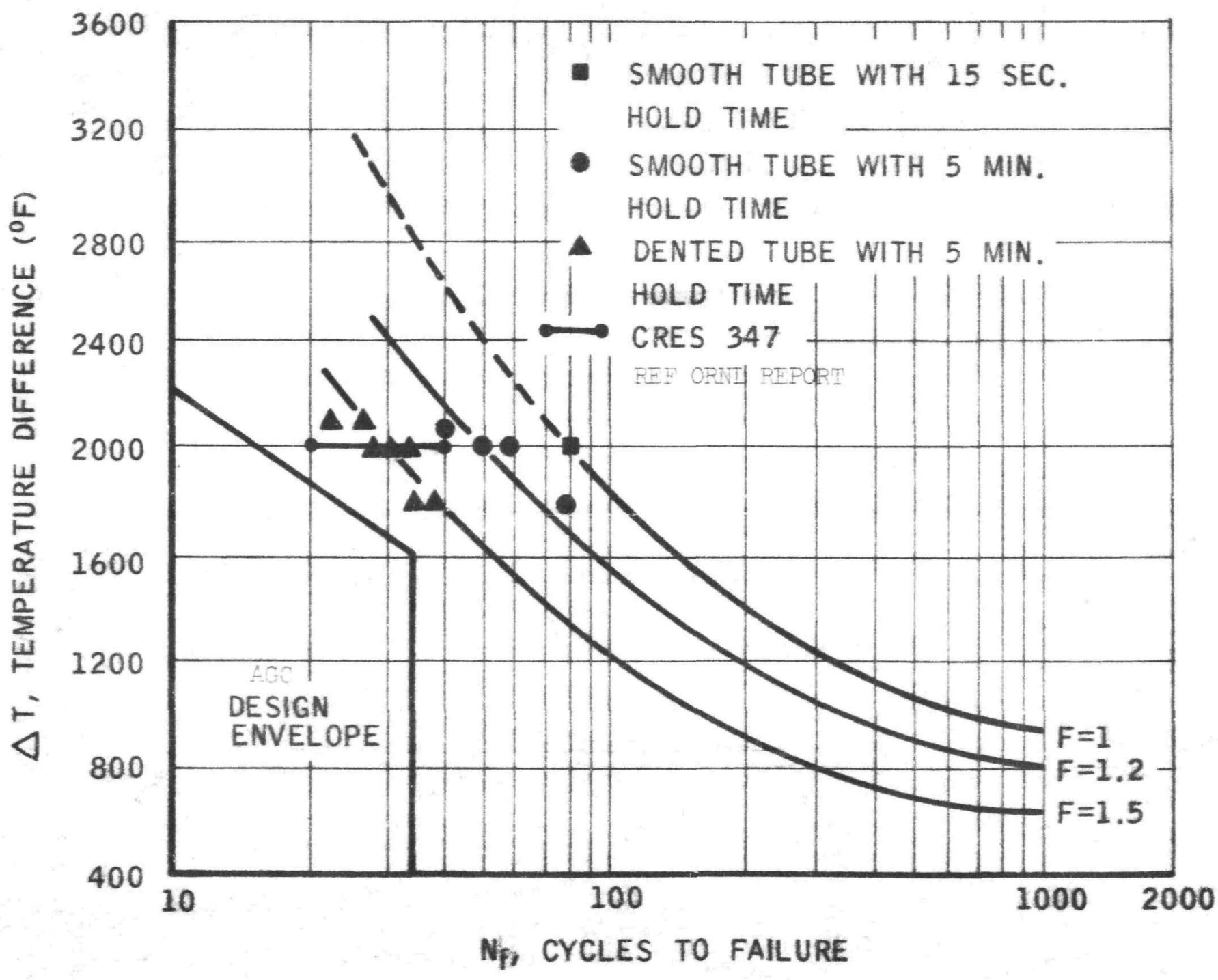

VELLUM IN TRADE STUDY REPORT 


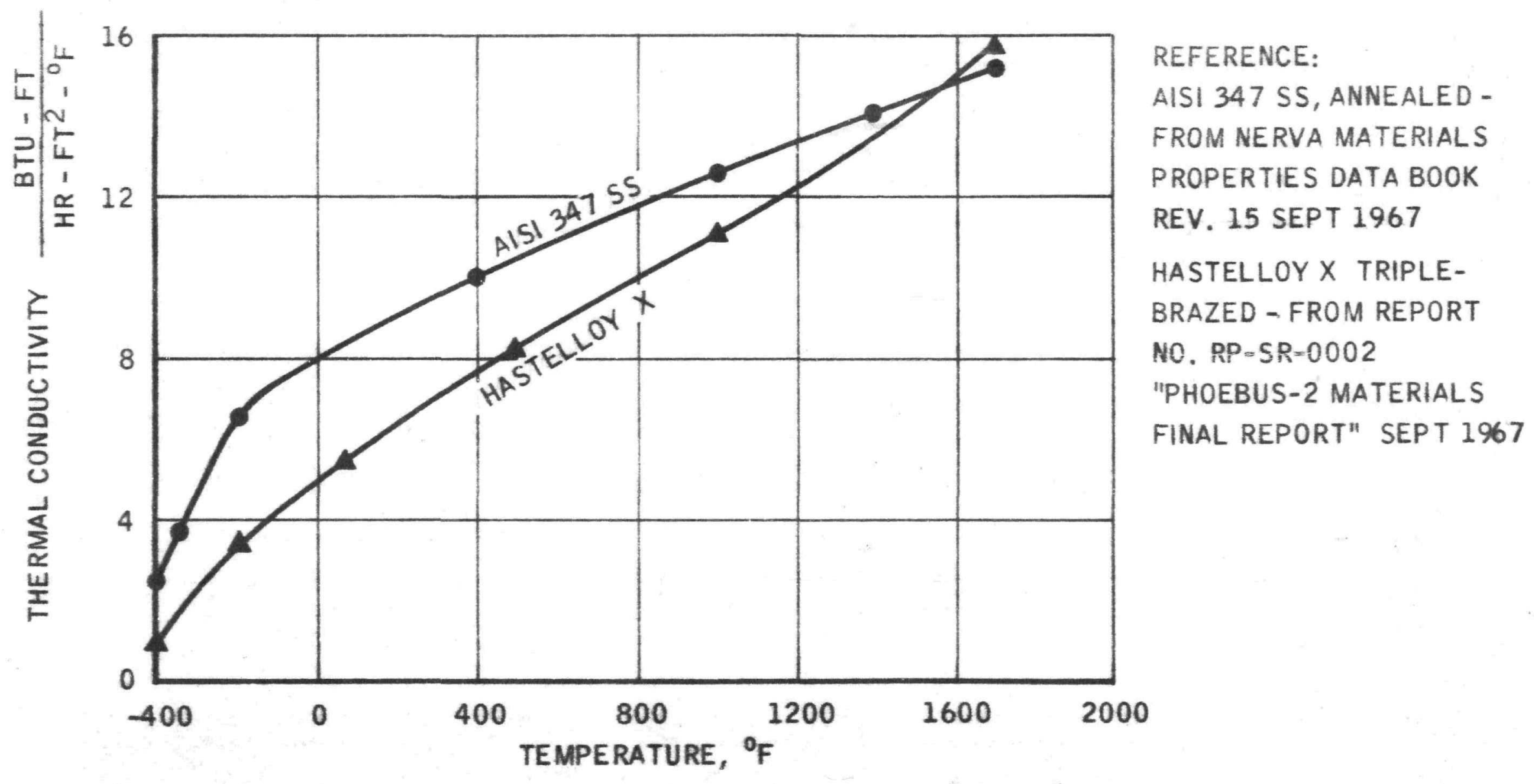




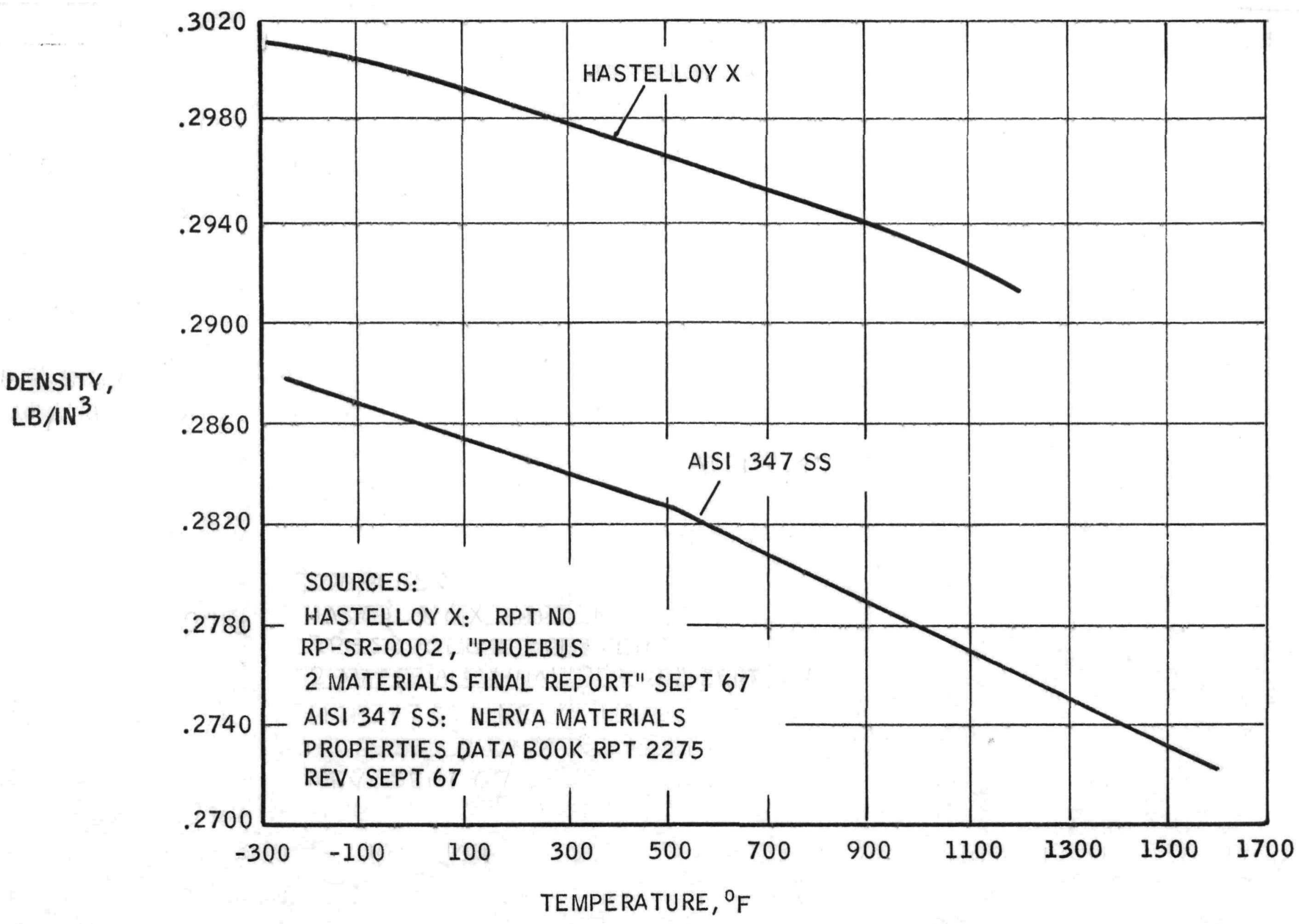


ANNEALED ROOM TEMPERATURE CRES 347 NOZZLE PROPERTIES *

\begin{tabular}{|c|c|c|c|c|}
\hline$S / N$ & $\begin{array}{c}0.2 \% \text { OFFSET } \\
\text { YIELD STRENGTH, } \\
\text { KSI }\end{array}$ & $\begin{array}{c}\text { ULTIMATE } \\
\text { TENSILE STRENGTH, } \\
\text { KSI }\end{array}$ & $\begin{array}{c}\text { ELONGA TION, } \\
\therefore\end{array}$ & $\begin{array}{l}\text { REDUCTION } \\
\text { OF AREA, } \\
\end{array}$ \\
\hline 26 & $\begin{array}{l}28.3 \\
29.8\end{array}$ & $\begin{array}{l}73.6 \\
76.1\end{array}$ & $\begin{array}{l}49 \\
50\end{array}$ & $\begin{array}{l}68 \\
67\end{array}$ \\
\hline 27 & $\begin{array}{l}31.3 \\
31.0 \\
29.7\end{array}$ & $\begin{array}{l}77.3 \\
79.1 \\
80.7\end{array}$ & $\begin{array}{l}54 \\
52 \\
48\end{array}$ & $\begin{array}{l}65 \\
66 \\
54\end{array}$ \\
\hline 28 & $\begin{array}{l}28.8 \\
27.5\end{array}$ & $\begin{array}{l}77.9 \\
76.2\end{array}$ & $\begin{array}{l}50 \\
51\end{array}$ & $\begin{array}{l}64 \\
67\end{array}$ \\
\hline 29 & $\begin{array}{l}26.9 \\
27.1\end{array}$ & $\begin{array}{l}73.1 \\
76.7\end{array}$ & $\begin{array}{l}56 \\
54\end{array}$ & $\begin{array}{l}64 \\
64\end{array}$ \\
\hline 30 & $\begin{array}{l}29.0 \\
29.6 \\
30.6 \\
31.0 \\
29.3 \\
28.2\end{array}$ & $\begin{array}{l}79.2 \\
79.7 \\
80.2 \\
79.2 \\
78.8 \\
78.5\end{array}$ & $\begin{array}{l}53 \\
53 \\
55 \\
59 \\
61 \\
60\end{array}$ & $\begin{array}{l}66 \\
65 \\
66 \\
66 \\
62 \\
64\end{array}$ \\
\hline
\end{tabular}


ANNEALED ROOM TEMPERATURE CRES 347 NOZZLE PROPERTIES * (CONT')

\begin{tabular}{|c|c|c|c|c|}
\hline$S / N$ & $\begin{array}{c}0.2 \% \text { OFFSET } \\
\text { YIELD STRENGTH, } \\
\text { KSI }\end{array}$ & $\begin{array}{c}\text { UL TIMATE } \\
\text { TENSILE STRENGTH } \\
\text { KSI }\end{array}$ & $\begin{array}{c}\text { ELONGATION, } \\
\%\end{array}$ & $\begin{array}{c}\text { REDUCTION } \\
\text { OF AREA } \\
\%\end{array}$ \\
\hline 31 & $\begin{array}{l}29.9 \\
29.4\end{array}$ & $\begin{array}{l}76.4 \\
76.2\end{array}$ & $\begin{array}{l}50 \\
53\end{array}$ & $\begin{array}{l}62 \\
66\end{array}$ \\
\hline 32 & $\begin{array}{l}29.7 \\
29.1 \\
28.8 \\
28.9\end{array}$ & $\begin{array}{l}77.0 \\
76.9 \\
75.9 \\
75.6\end{array}$ & $\begin{array}{l}50 \\
51 \\
51 \\
46\end{array}$ & $\begin{array}{l}57 \\
55 \\
48 \\
48\end{array}$ \\
\hline 33 & $\begin{array}{l}30.8 \\
29.8 \\
29.6 \\
29.2\end{array}$ & $\begin{array}{l}82.5 \\
79.7 \\
80.7 \\
80.9\end{array}$ & $\begin{array}{l}43 \\
47 \\
50 \\
46\end{array}$ & $\begin{array}{l}55 \\
60 \\
60 \\
61\end{array}$ \\
\hline
\end{tabular}

* OPEN DIE FORGING

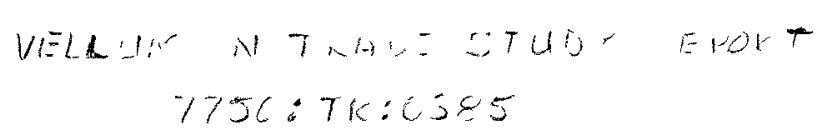




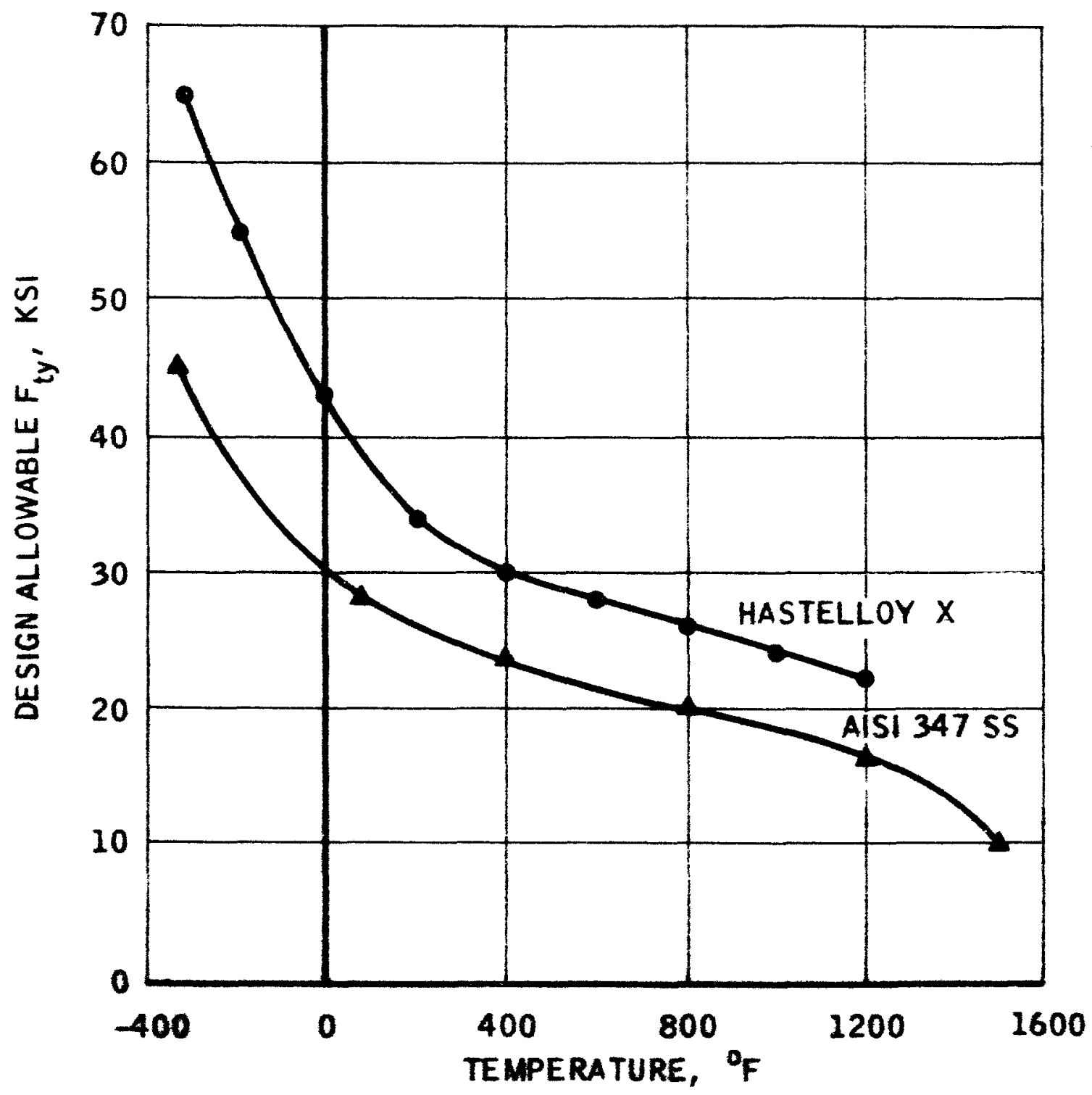

REFERENCE:

HASTELLOY X FORGING TRIPLEBRAZED, FROM REPT. NO, RP-SR0002 "PHOEBUS-2 MATERIALS FINAL REPT." SEPT 1967. CURVE IS -20 LIMIT.

AISI 347 FORGING, ANNEALED FROM NERVA MATERIALS PROPERTIES DATA BOOK. SEPT 1967 REV. AND MEMO SAK:sad:7340. M5710 DATED $1 / 24 / 68$. CLRVE CORRESPONDS TO MINIMUM TEST VALUE OBTAINED AT ROOM TEMP. 


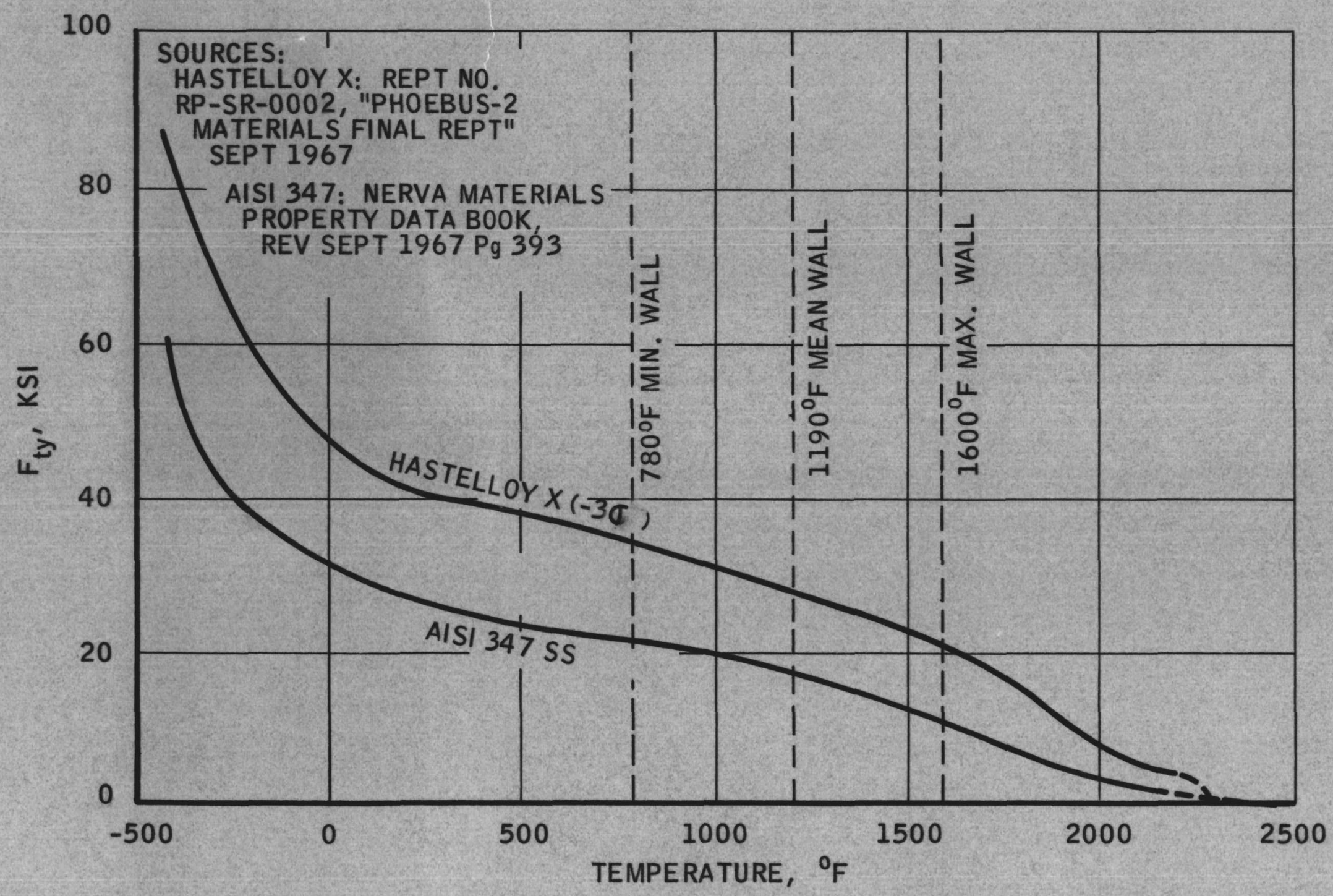




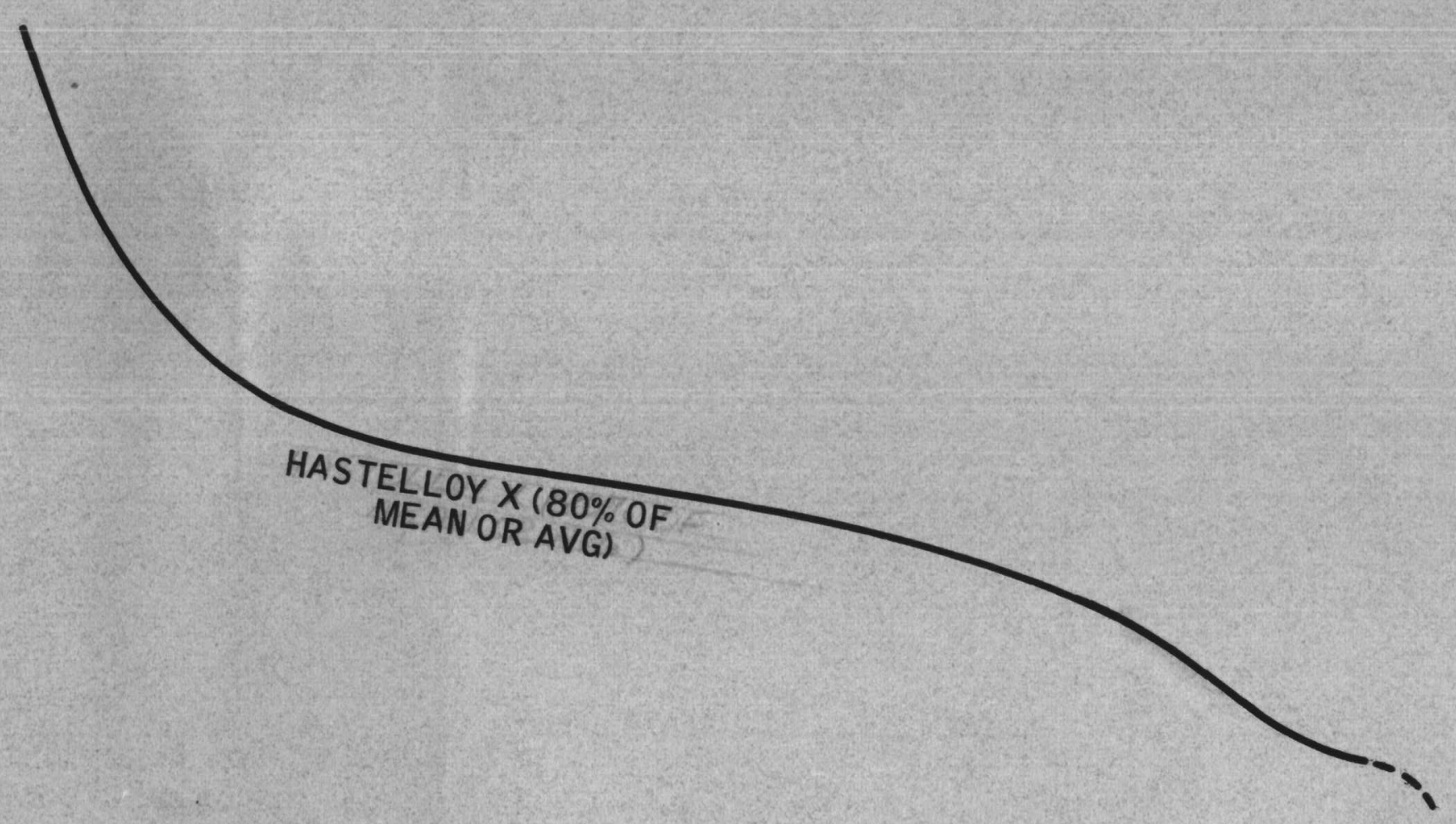




\section{FABRICATION COST SAVINGS}

\begin{tabular}{lccr} 
ITEM & CRES 347 & HASTELLOY X & \multicolumn{1}{c}{$\Delta$} \\
\cline { 2 - 3 } FORGING & $\$ 24,000$ & $\$ 56,000$ & $\$ 32,000$ \\
COOLANT CHANNELS & 26,000 & 29,000 & 3,000 \\
TORI \& FLANGES & 10,000 & 30,000 & 20,000 \\
WELDMENT FORGING & - & 15,900 & 15,900 \\
CONTOUR MACHINING & 40,000 & 49,000 & 9,000 \\
GROOVE \& SCALLOP & 36,000 & 43,900 & $\mathbf{7 , 9 0 0}$ \\
WELDMENT, TORI & 10,500 & 11,300 & 800 \\
\hline GROOVE \& SCALLOP (2 SETS) & $\$ 70,000$ & $\$ 8,600$
\end{tabular}

ALL OTHER TOOLING APPROXIMATELY THE SAME FOR EITHER NOZZLE 


\section{SCHEDULE}

ITEM

FORGING

INGOT

COMPLETE FORGE

FABRICATION

WELD SECTORS

GROOVE, SCALLOP

\section{CRES 347}

15

11

\section{HASTELLOY $X$}

17

13

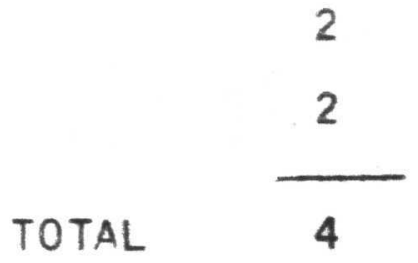

5

9

TOTAL

TOTAL FAB SPAN TIME REDUCTION

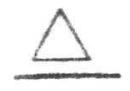

5

1

6

10

NOTE: ALL SPAN TIMES IN WEEKS

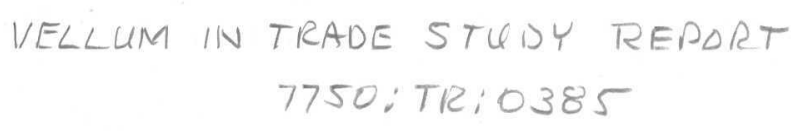




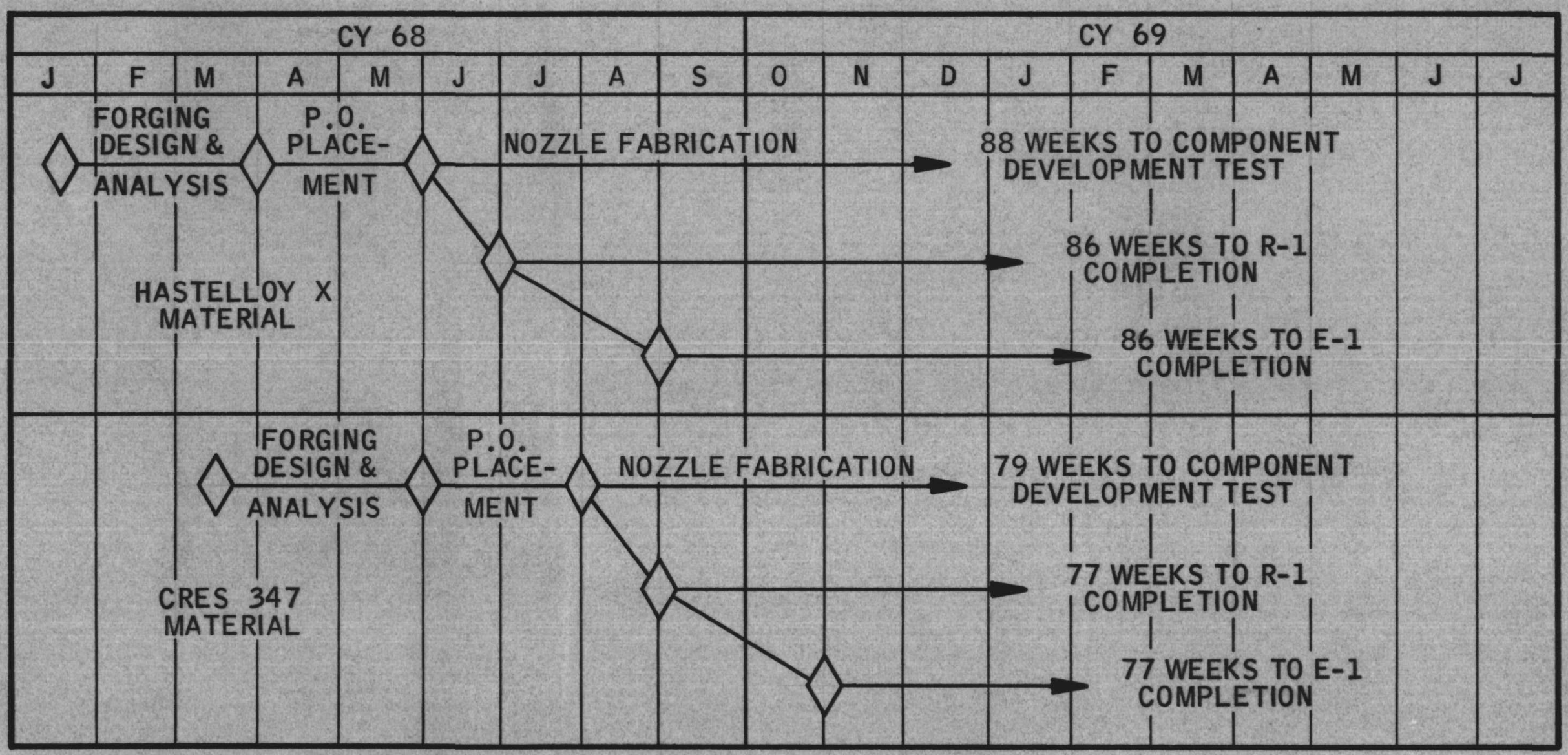

ENGINE DESIGN \& ANALYSIS LOGIC

1 WEEK: PREPARE LAYOUT

2 WEEKS: NUCLEAR ANALYSIS

2 WEEKS: THERMAL ANALYSIS

1 WEEK: STRESS ANALYSIS

2 WEEKS: FAB DWG PREPARATION \& RELEASE

2 WEEKS: DESIGN REVIEW \& APPROVAL

TOTAL 10 WEEKS 
1. BOTH CRES 347 \& HASTELLOY ' $X$ ' JACKETS ARE CAPABLE OF BEING UPGRADED TO IO6K THRUST.

2. A CRES 347 OR A HASTELLOY X JACKET WILL OPERATE AT APPROXIMATELY THE SAME TEMPERATURE UNDER ALL CONDITIONS.

3. THE NOZZLE WEIGHT WILL BE APPROXIMATELY THE SAME FOR CRES 347 OR HASTELLOY $X$.

4. A JACKET WITH EITHER MATERIAL MAY BE DESIGNED TO THE SAME MARGIN OF SAFETY.

5. CHEMICAL SIMULATION TESTS AND CALCULATED VALUES HAVE INDICATED CRES 347 IS A MORE RELIABLE TUBE MATERIAL.

6. SIGNIFICANT COST AND SCHEDULE SAVINGS CAN BE REALIZED WITH CRES 347.

7. BRAZING PROBLEMS EXPERIENCED ON PHOEBUS WILL BE ELIMINATED BY USING CRES 347.

8. ADDITIONAL MATERIALS DEVELOPMENT WILL NOT HAVE TO BE DONE TO EVALUATE THE LOW ALUMINUM CONTENT HASTELLOY X IF CRES 347 IS USED.

9. AGC RECOMMENDS THE USE OF CRES 347 AS A NOZZLE JACKET MATERIAL. 
Viewgraphs not used in presen tation. 


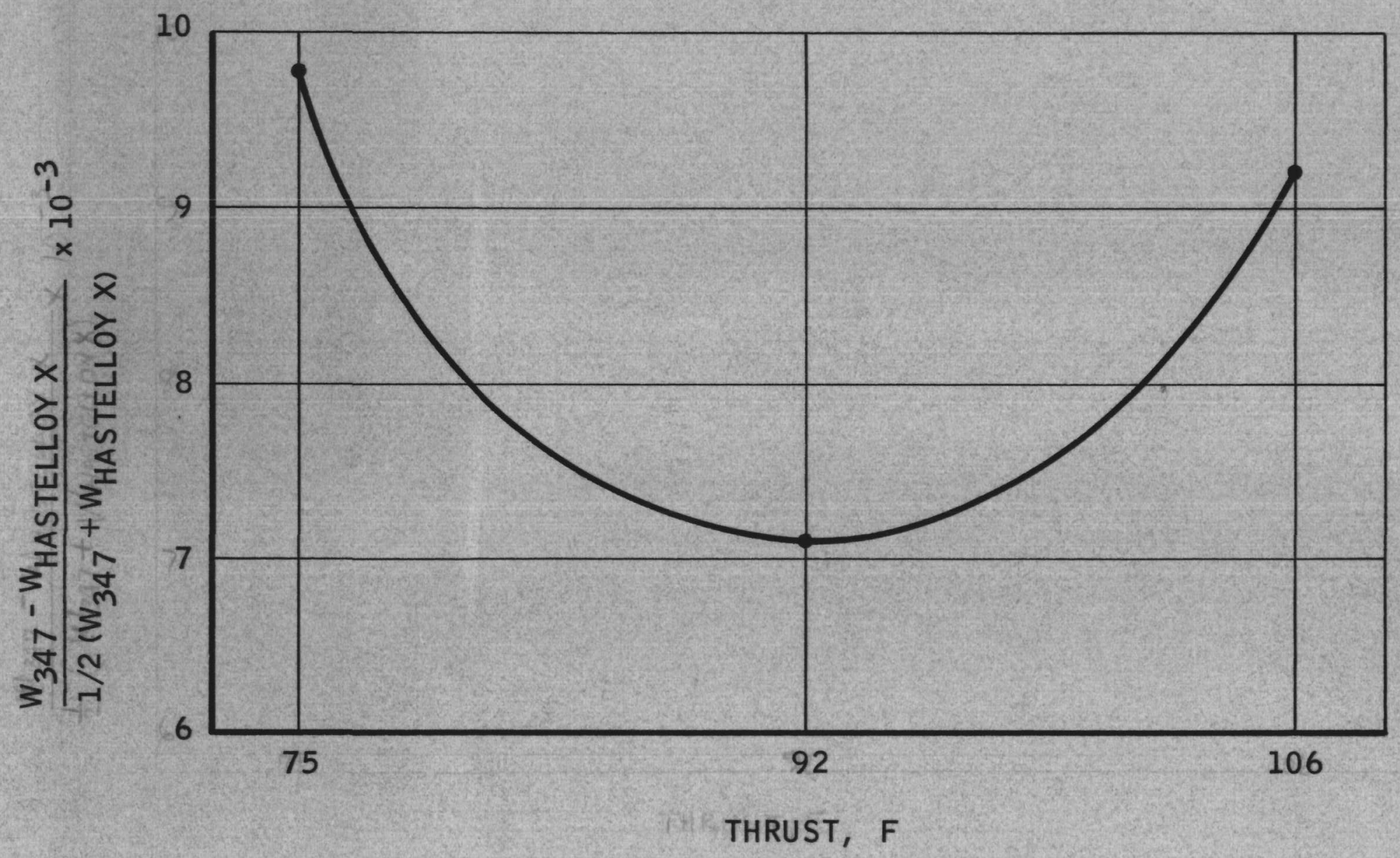




\section{RELIABILITY}

\section{RELIABILITY CLASSIFICATION}

BEST ESTIMATE (QUALITATIVE)

HISTORICAL

UPPER LIMIT

\section{NOZZLE RELIABILITY VALUES}

\section{CRES 347}

.9996910

.999000

.999900

\section{HASTELLOY $X$}

.999478

.998310

.999831

NERVA NOZZLE RELIABILITY GOAL (PRELIMINARY)

$$
\begin{array}{ll}
.995 & \text { ENGINE GOAL } \\
.99987 & \text { NOZZLE GOAL }
\end{array}
$$

CONCLUSIONS:

CRES 347 HAS A SLIGHT NUMERICAL RELIABILITY ADVANTAGE. HOWEVER, BOTH MATERIALS APPEAR TO HAVE THE POTENTIAL FOR MEETING THE NOZZLE RELIABILITY GOAL 


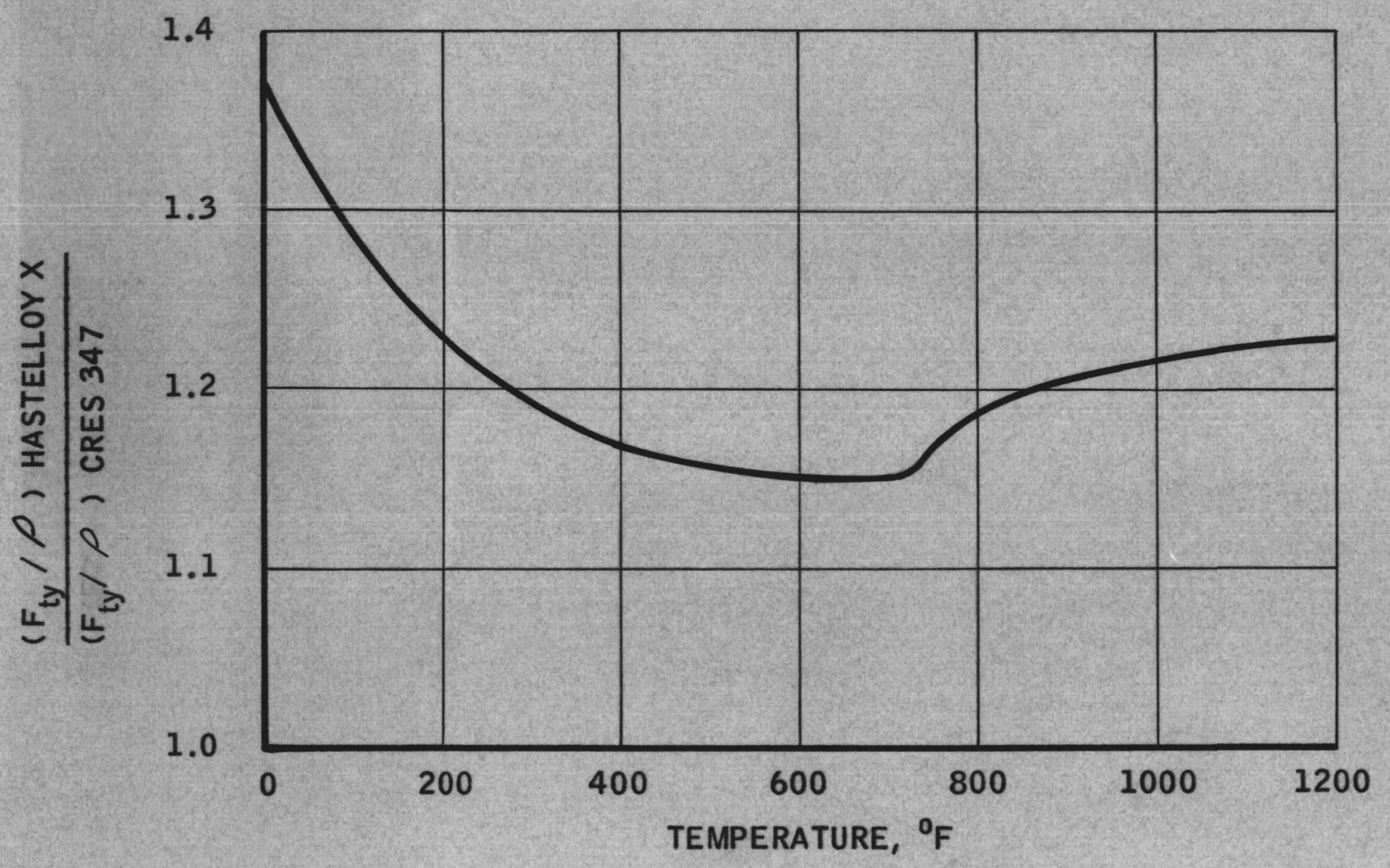




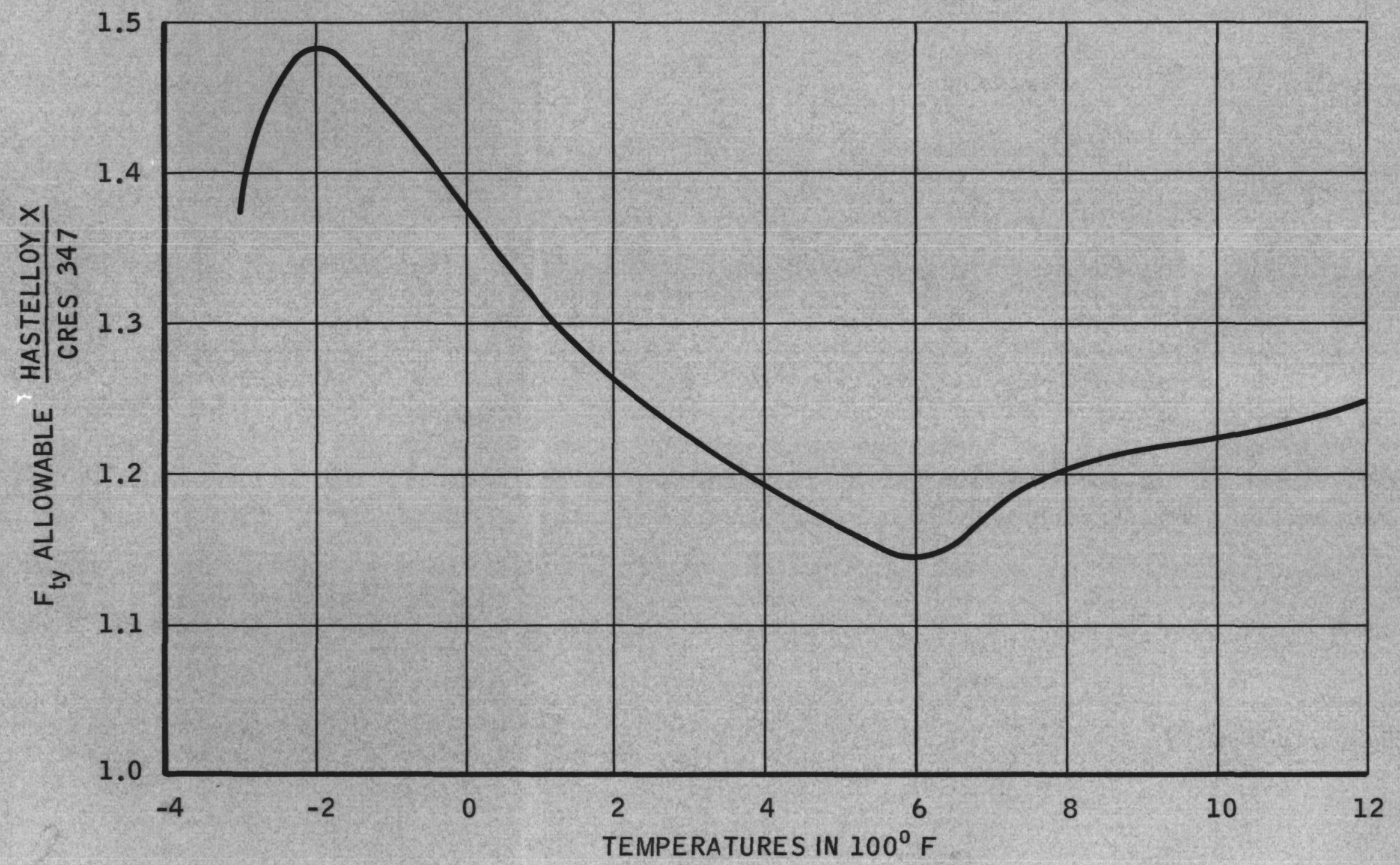


NRO EFFECTS OF THERMAL NEUTRON EXPOSURE AT AMBIENT TEMPERATURE

ON ELONGATION OF AISI-347 - 0.015 IN. SHEET






\section{W NRO}

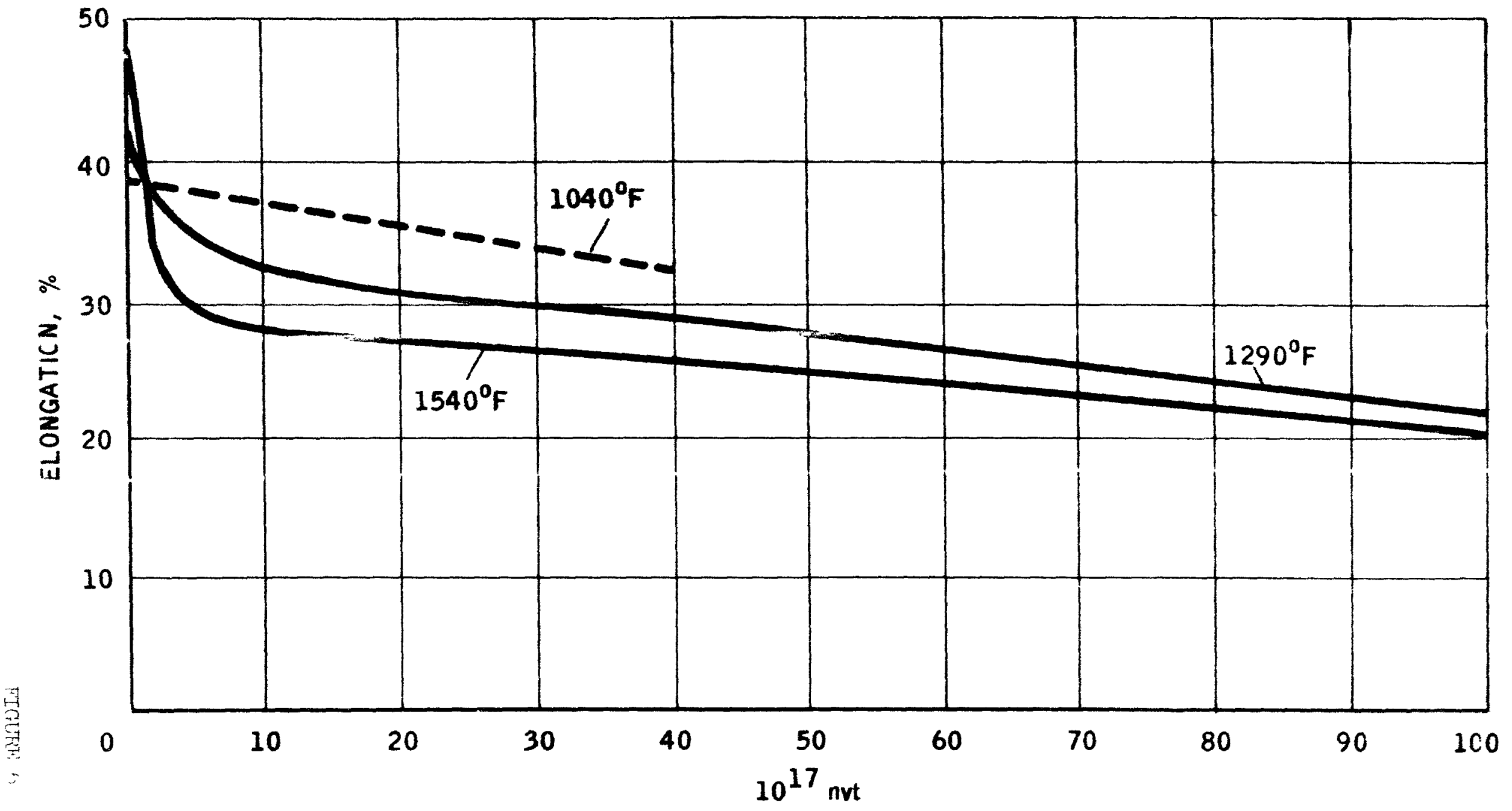


$268-190$

Comparuain of Allozy thermal Fotigue Resiatanee MO MHSTER SeLLGM 

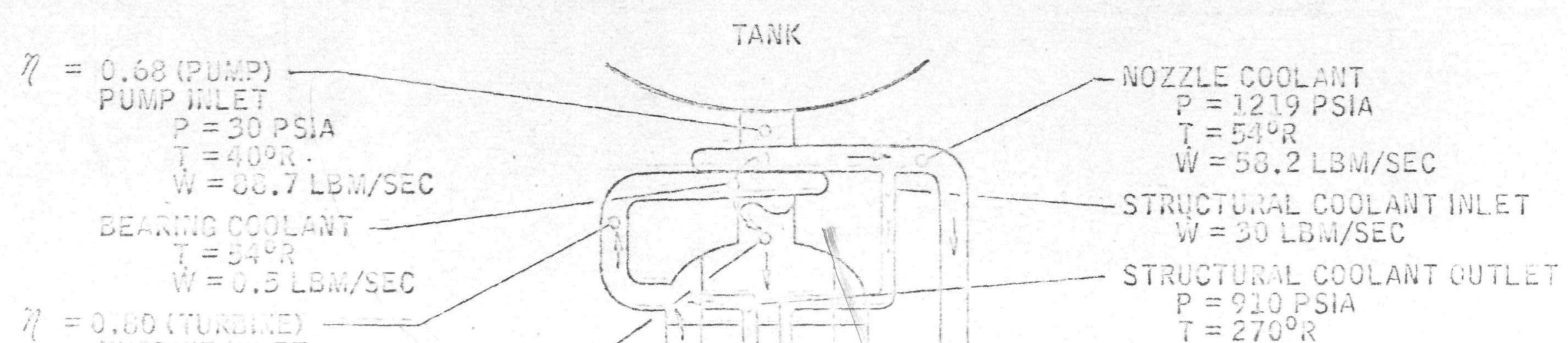

1 $-0,00(4,2)$

T

$=3030$ ?

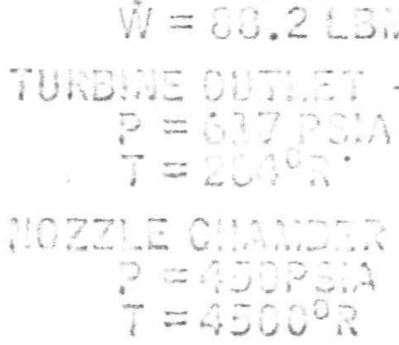


agc. ND

NERVA FULL FLOW ENGINE

PRESSURE VESSEL CLOSURE CONCEPT

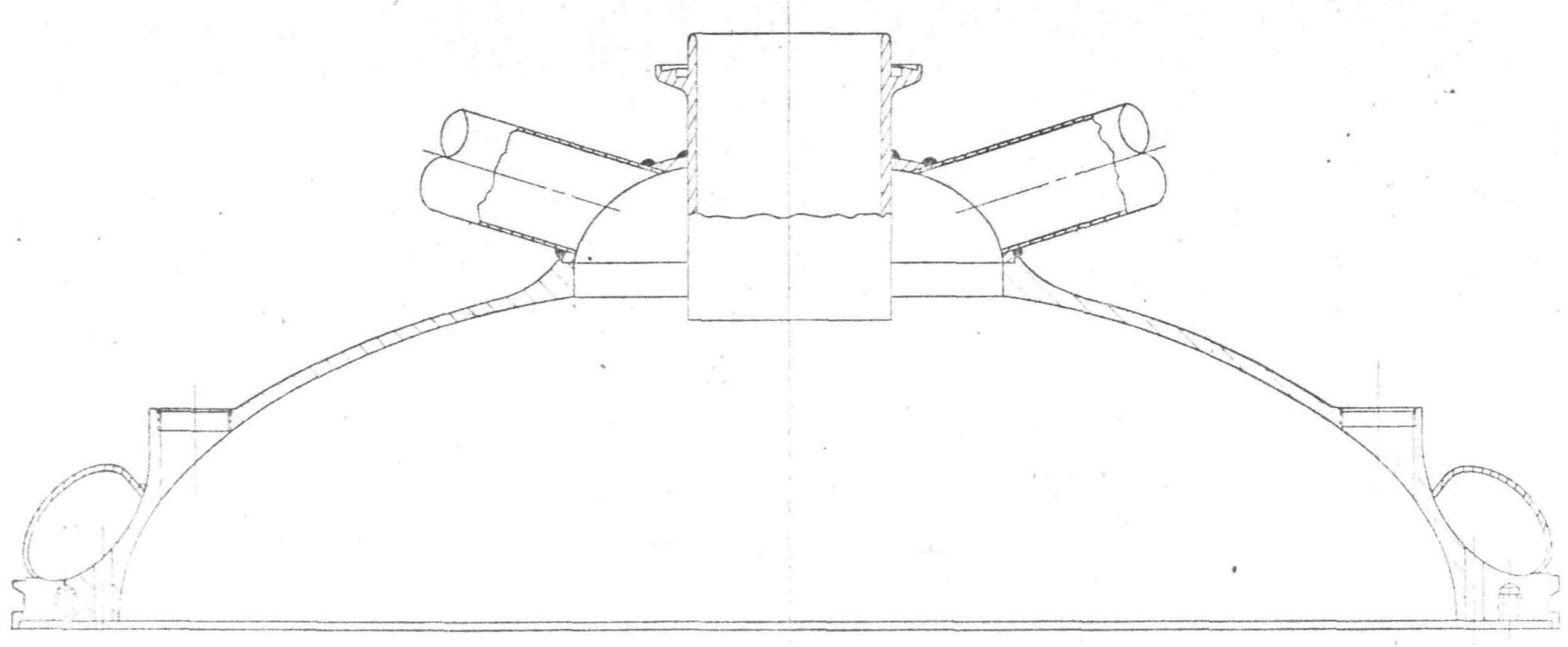


<smiles>[3H][Te]</smiles> 


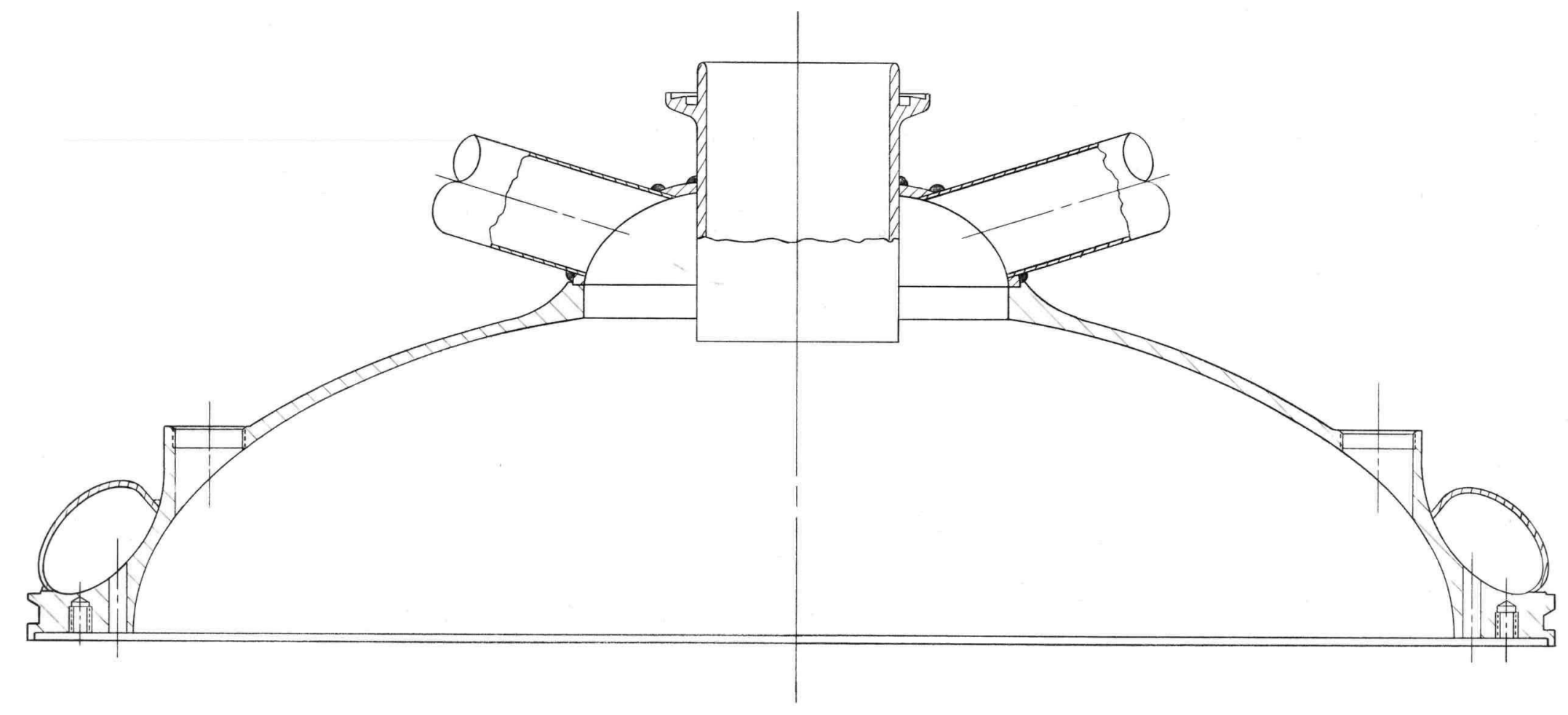


NERVA FULL FLOW ENGINE

PRESSURE VESSEL CLOSURE TO CYLINDER FLOW CONCEPT NO. I
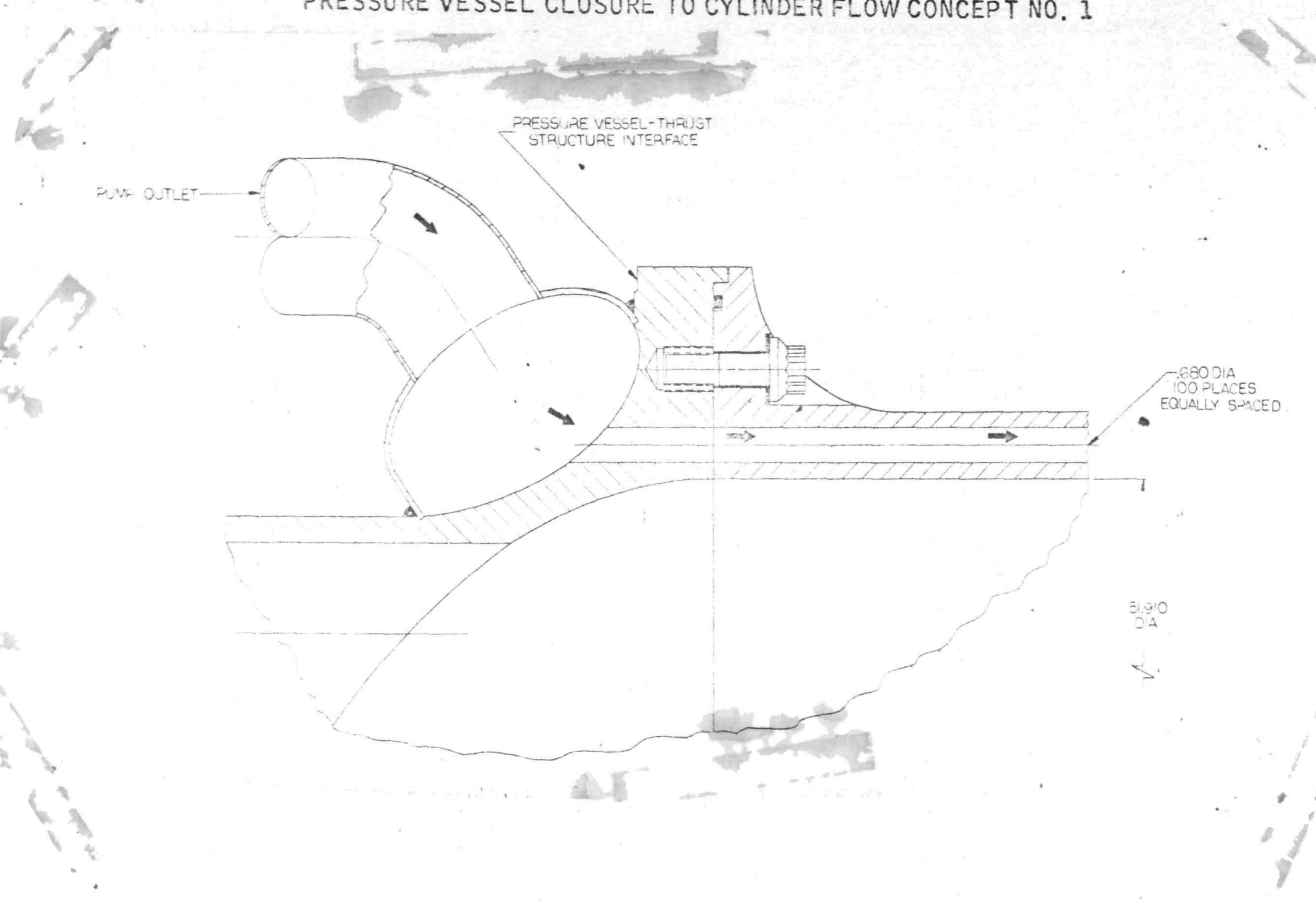


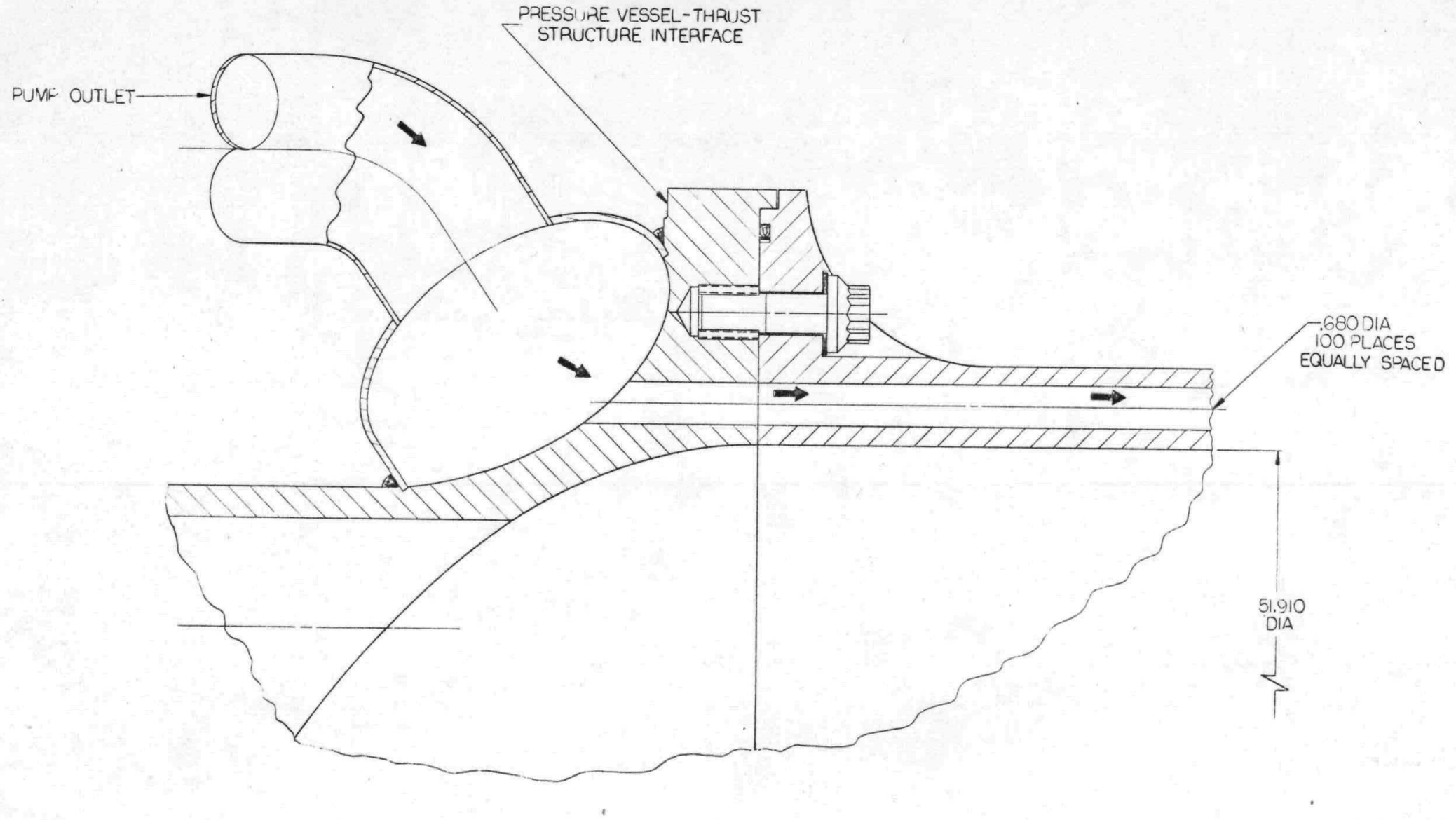




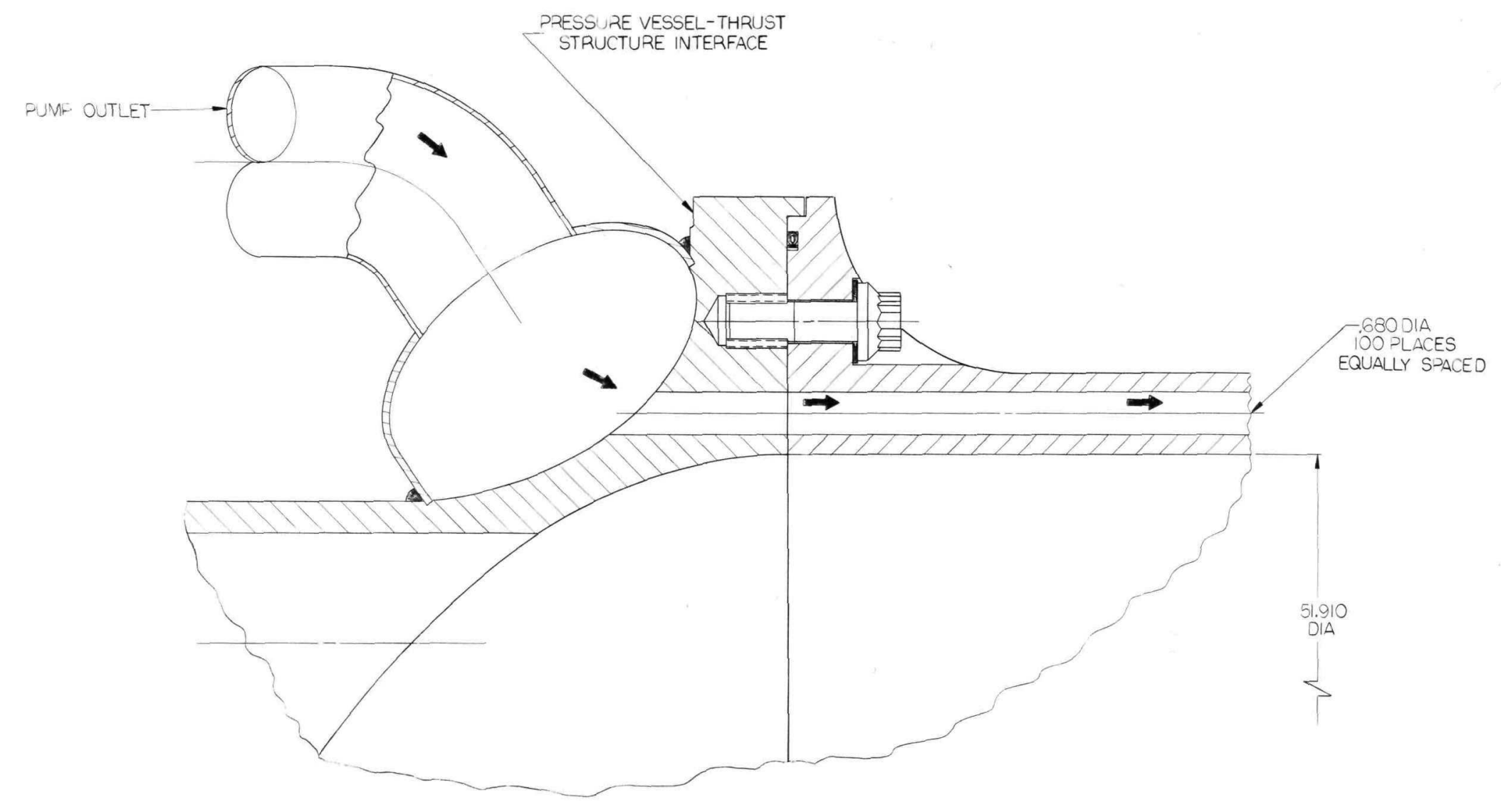




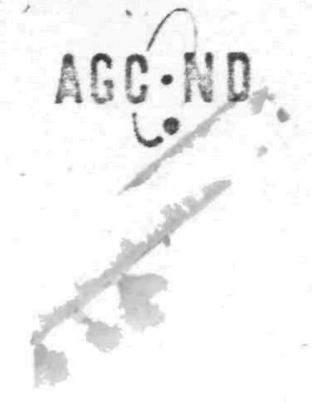

NERVA FULL FLOW ENGINE

PRESSURE VESSEL CLOSURE TO CYLINDER FLOW CONCEPT NO. 2

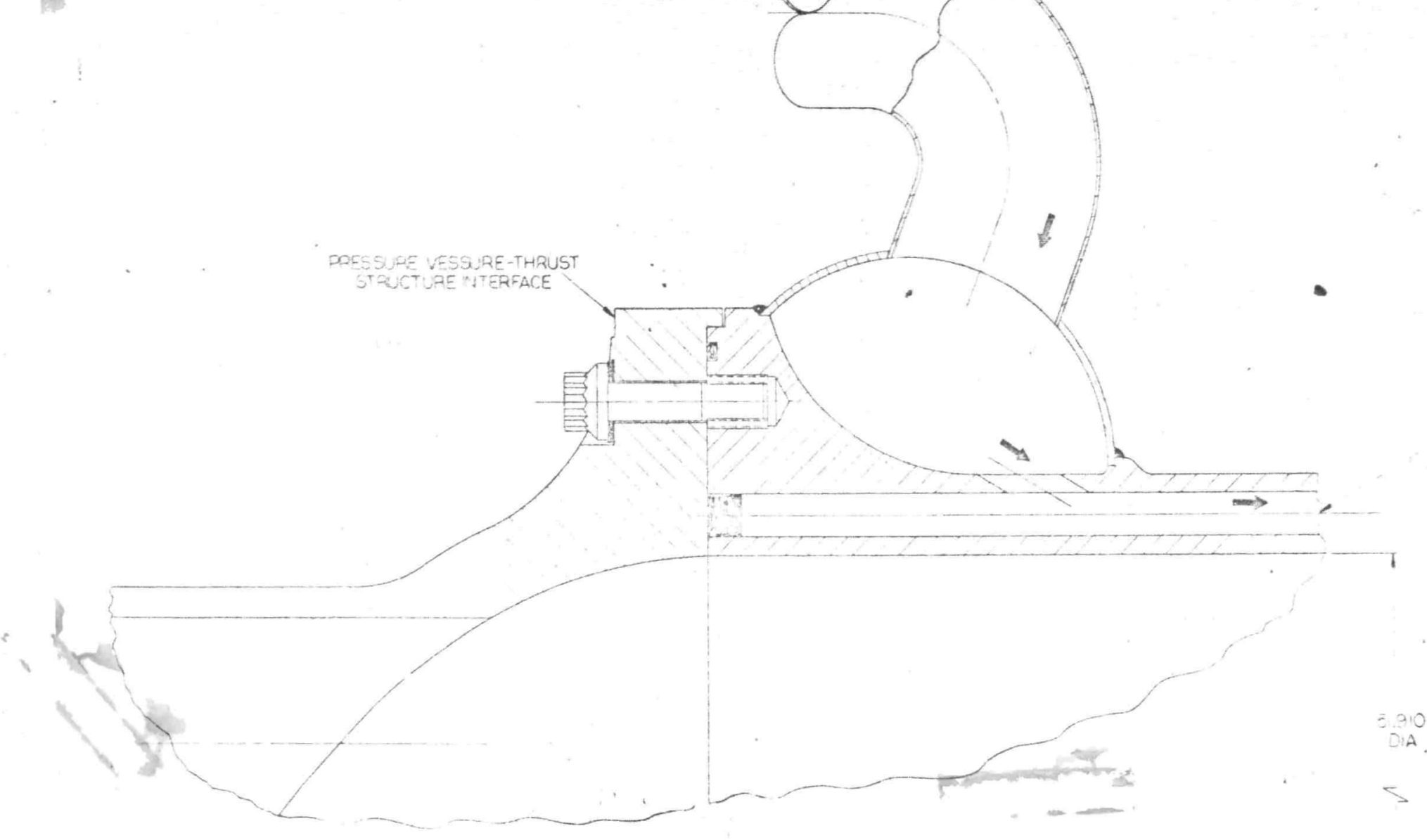




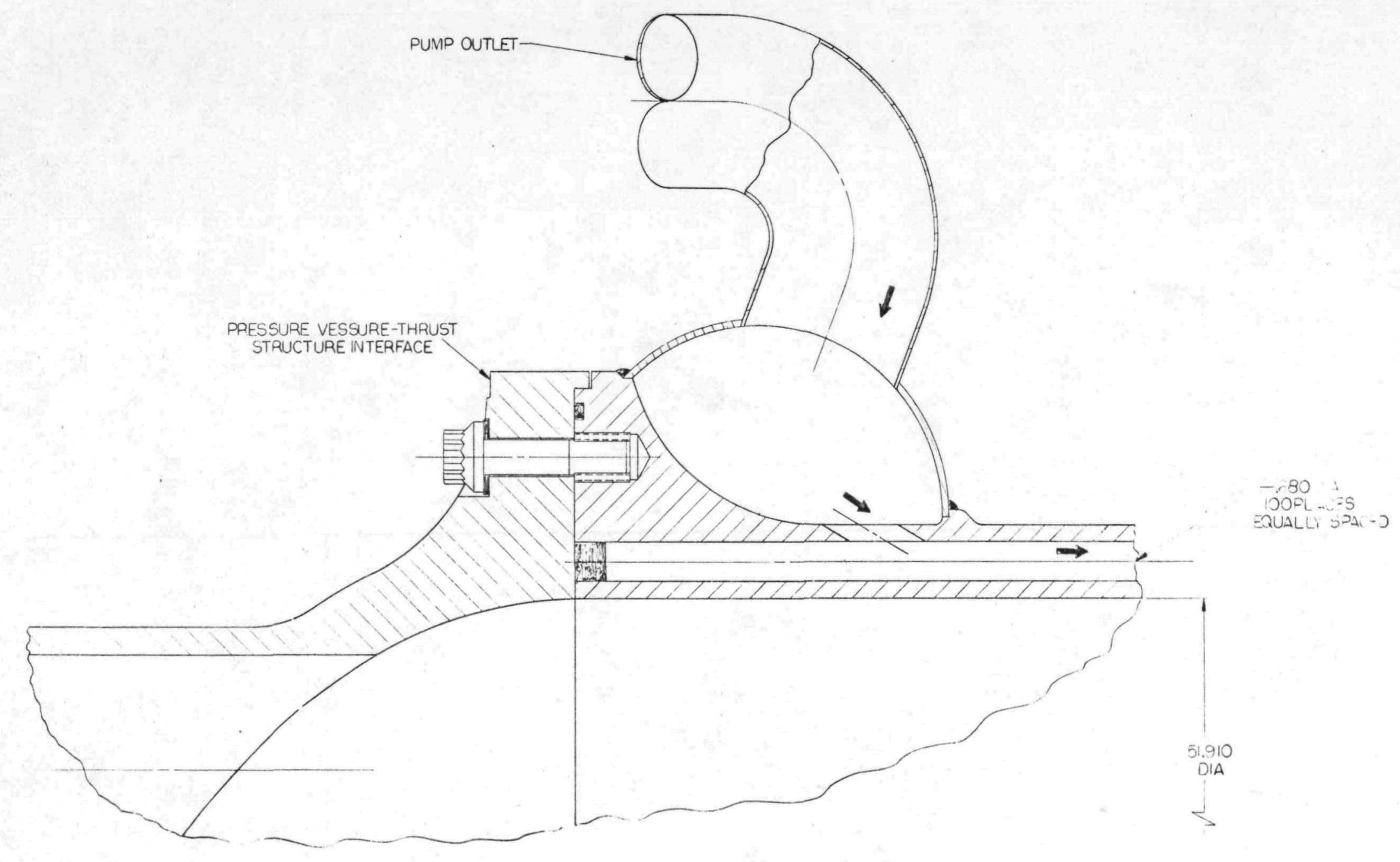




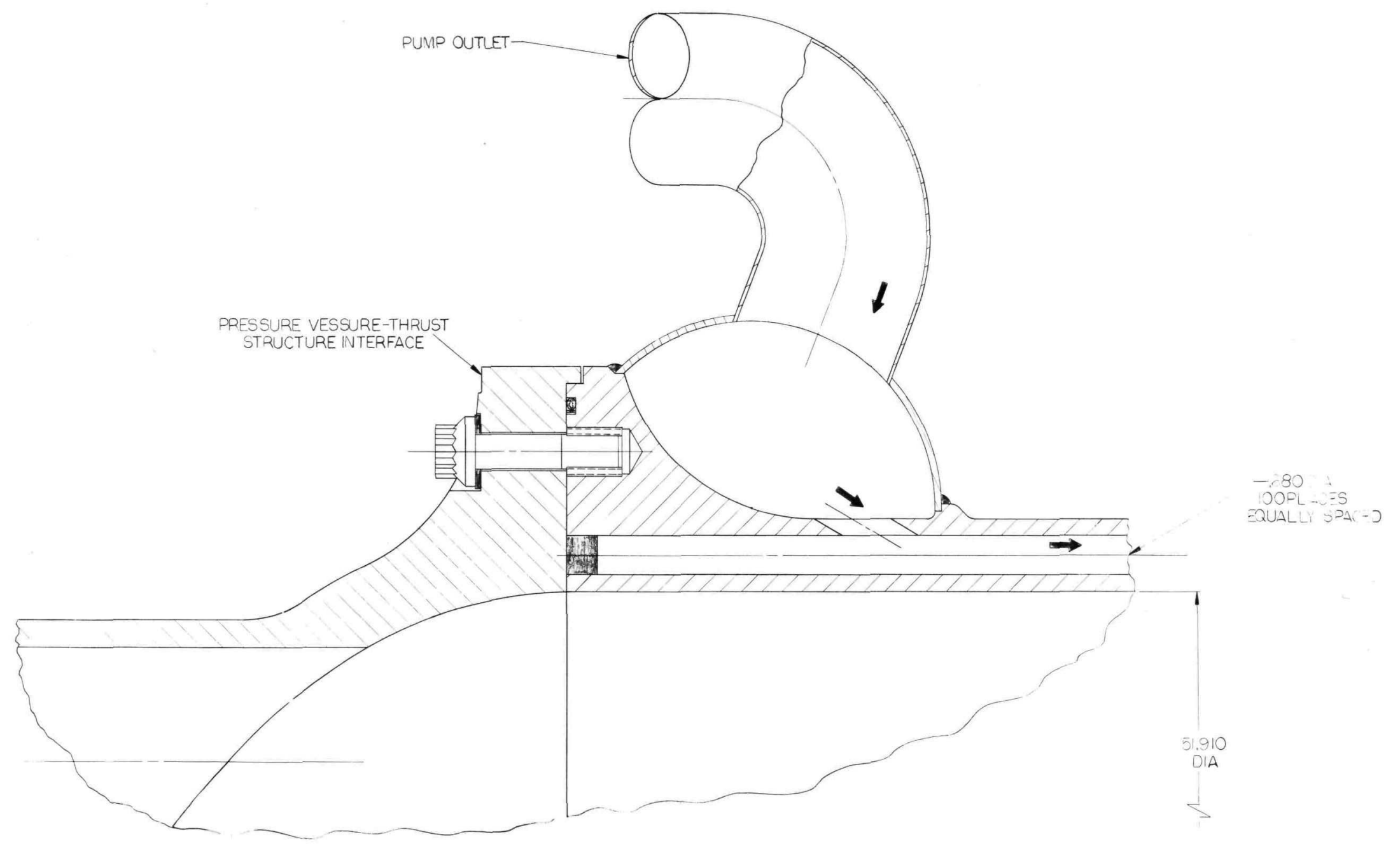




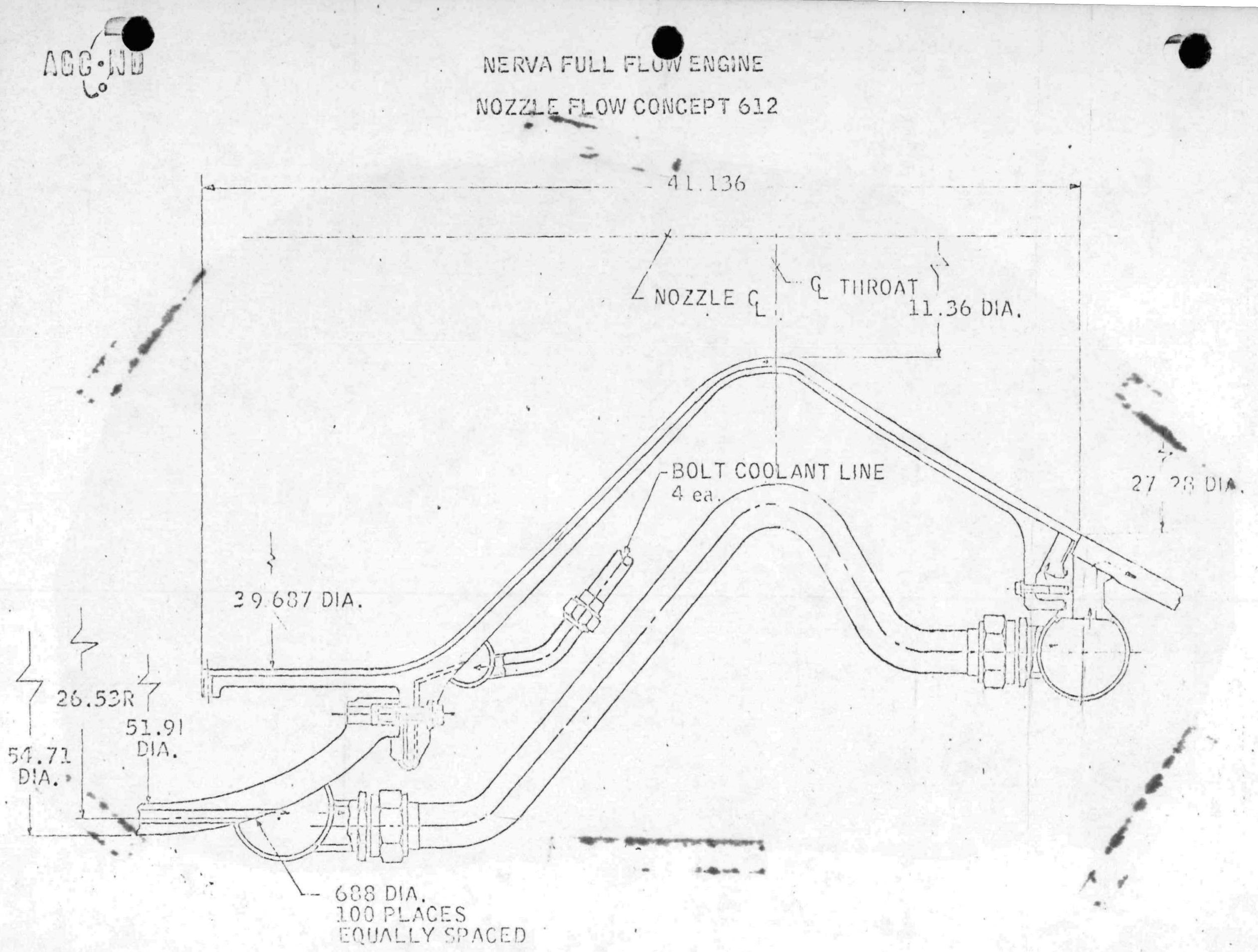


NERVA FULL FLOW ENGINE

- NOZZLE FLOW CONCEPT 614

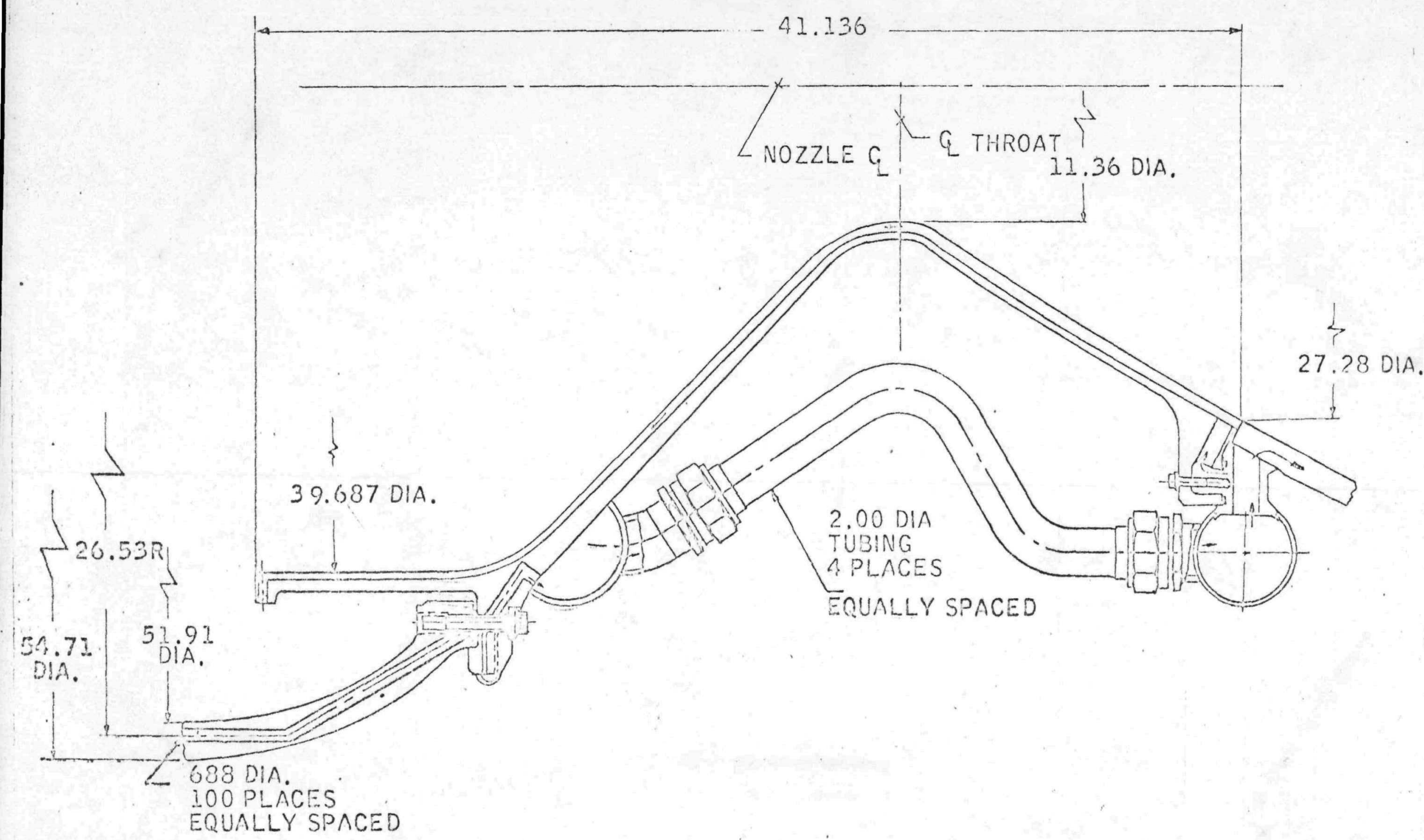




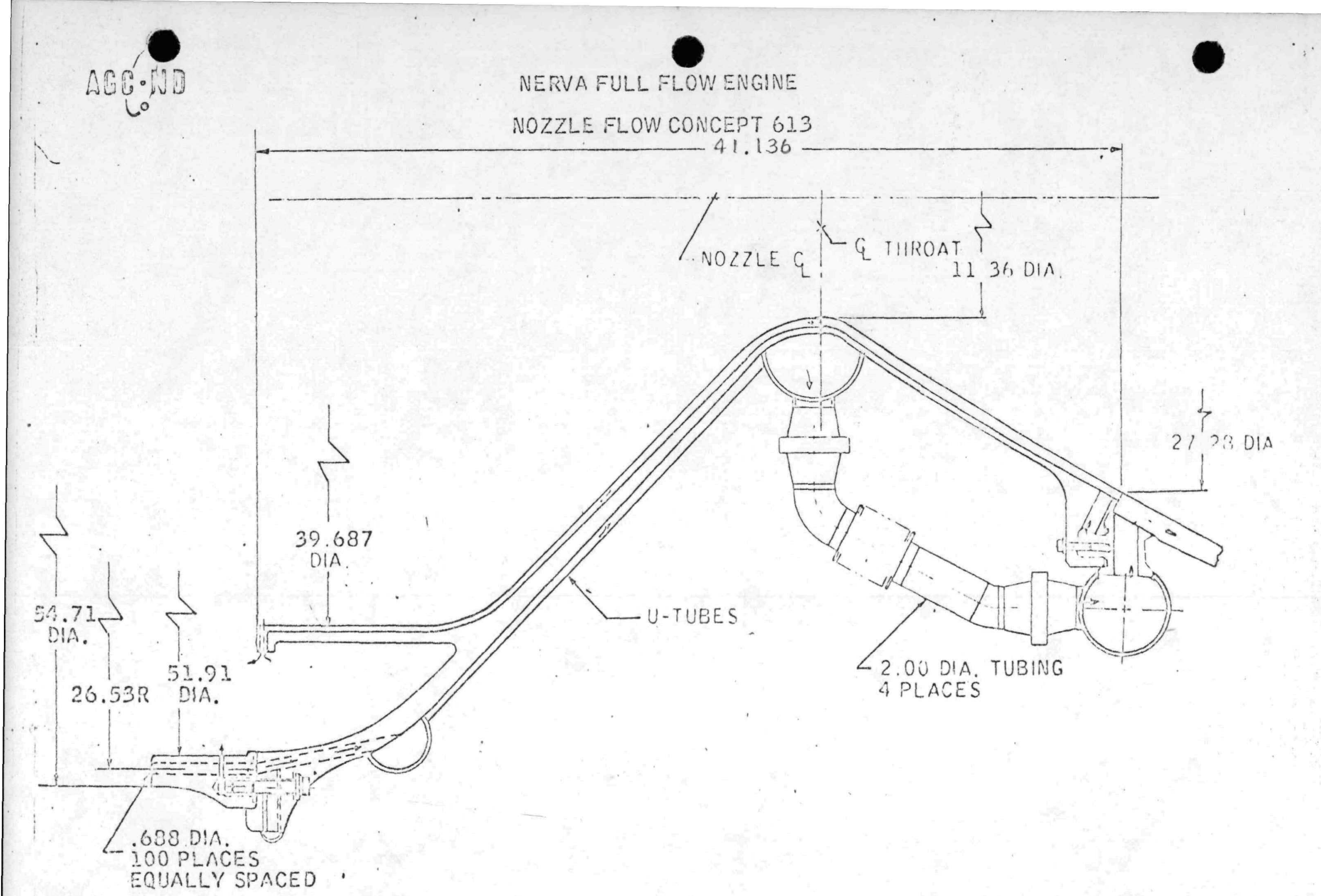




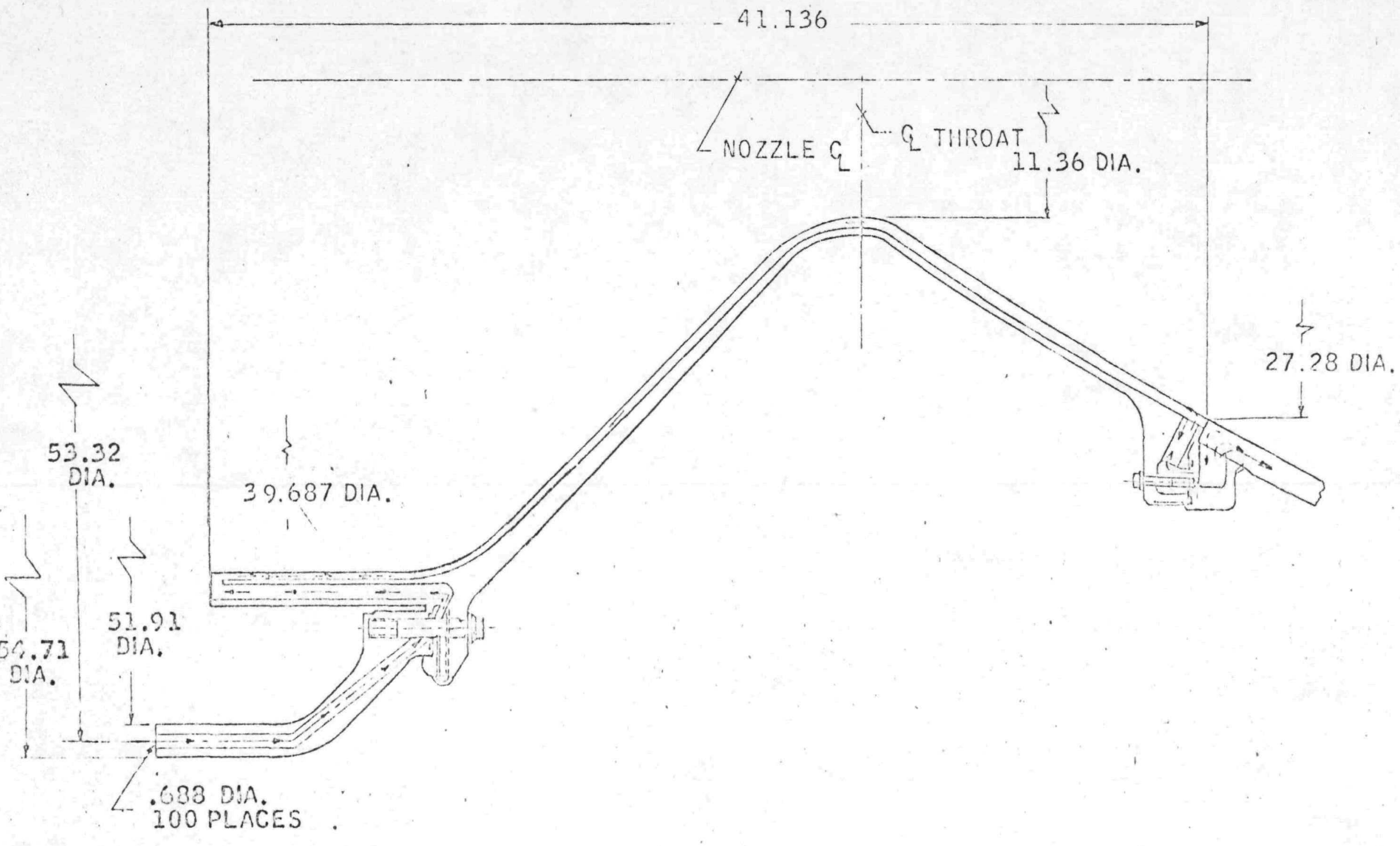




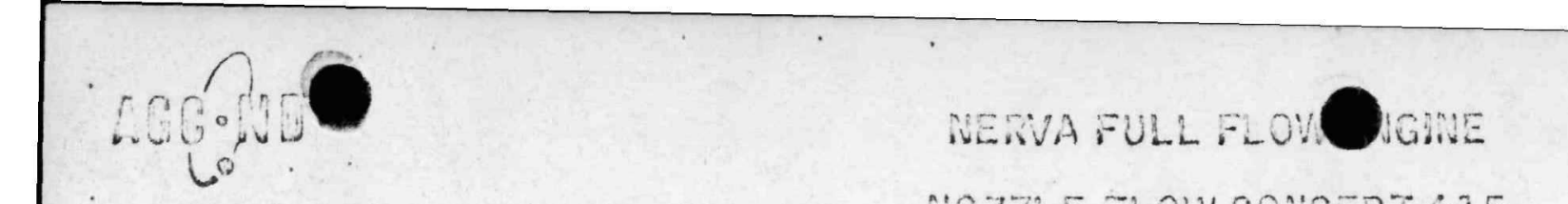

NOZZLE FLOW CONCEPT 615

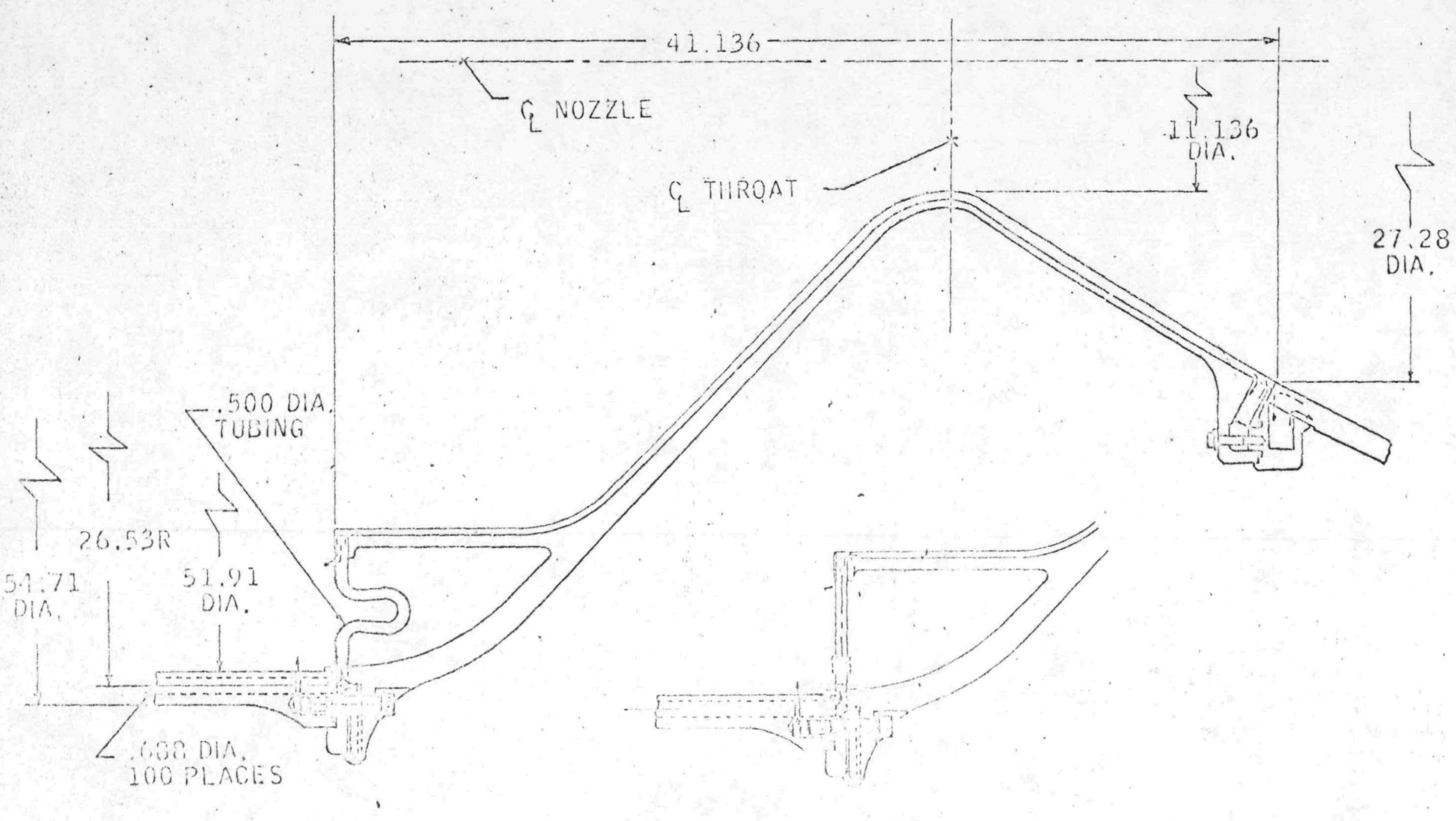

ALTERNATE TUBE DESIGN 


\section{MARGIN OF SAFETY VS TUBE $\triangle P$}

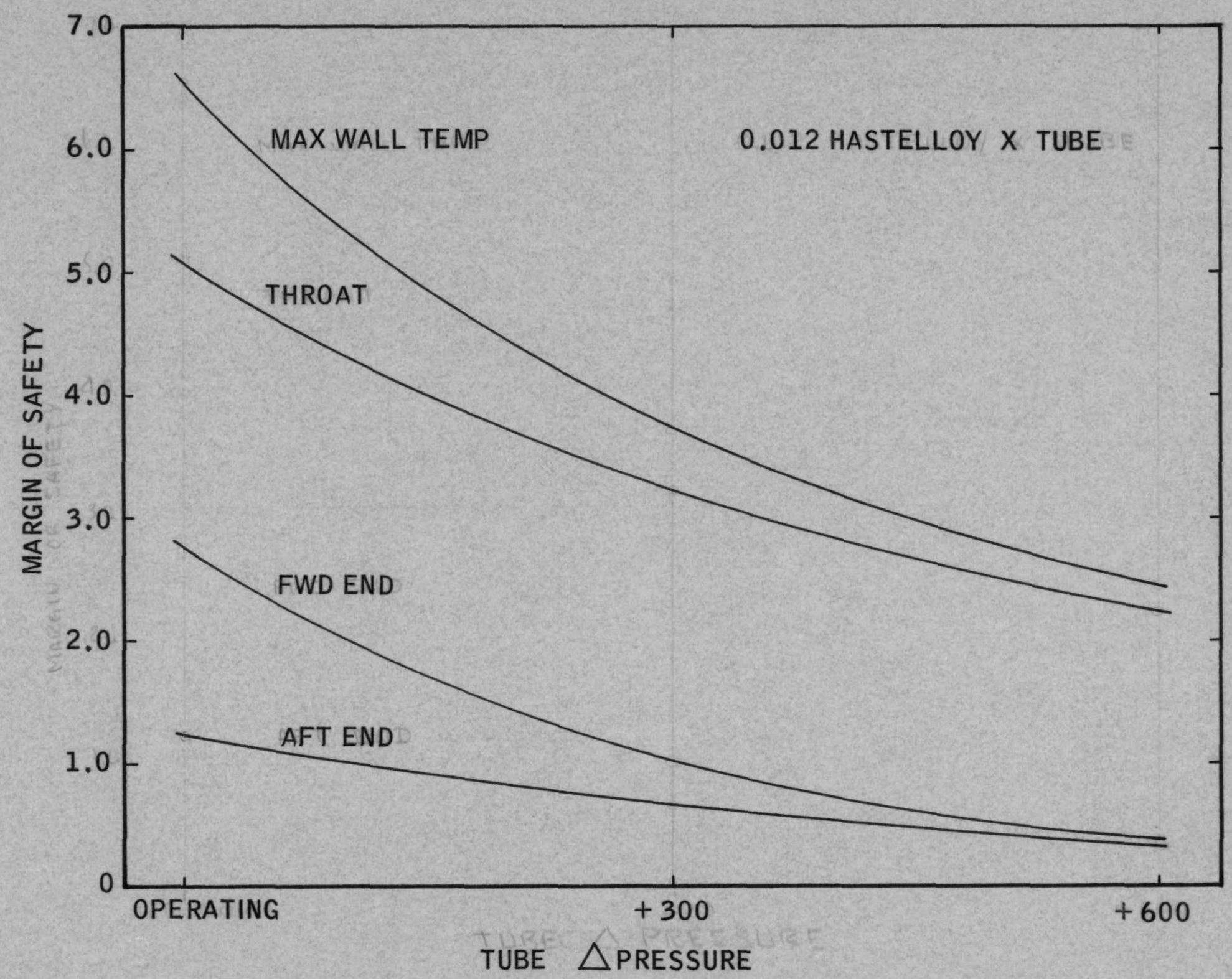




\section{AGC.ND}

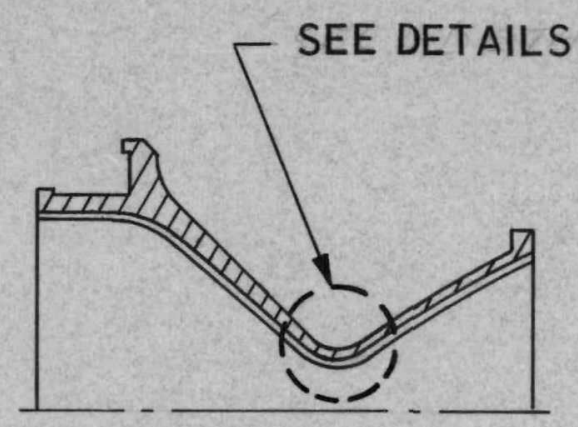

NERVA NOZZLE
TWO - PASS

COOLANT FLOW ENHANCEMENT EFFECT
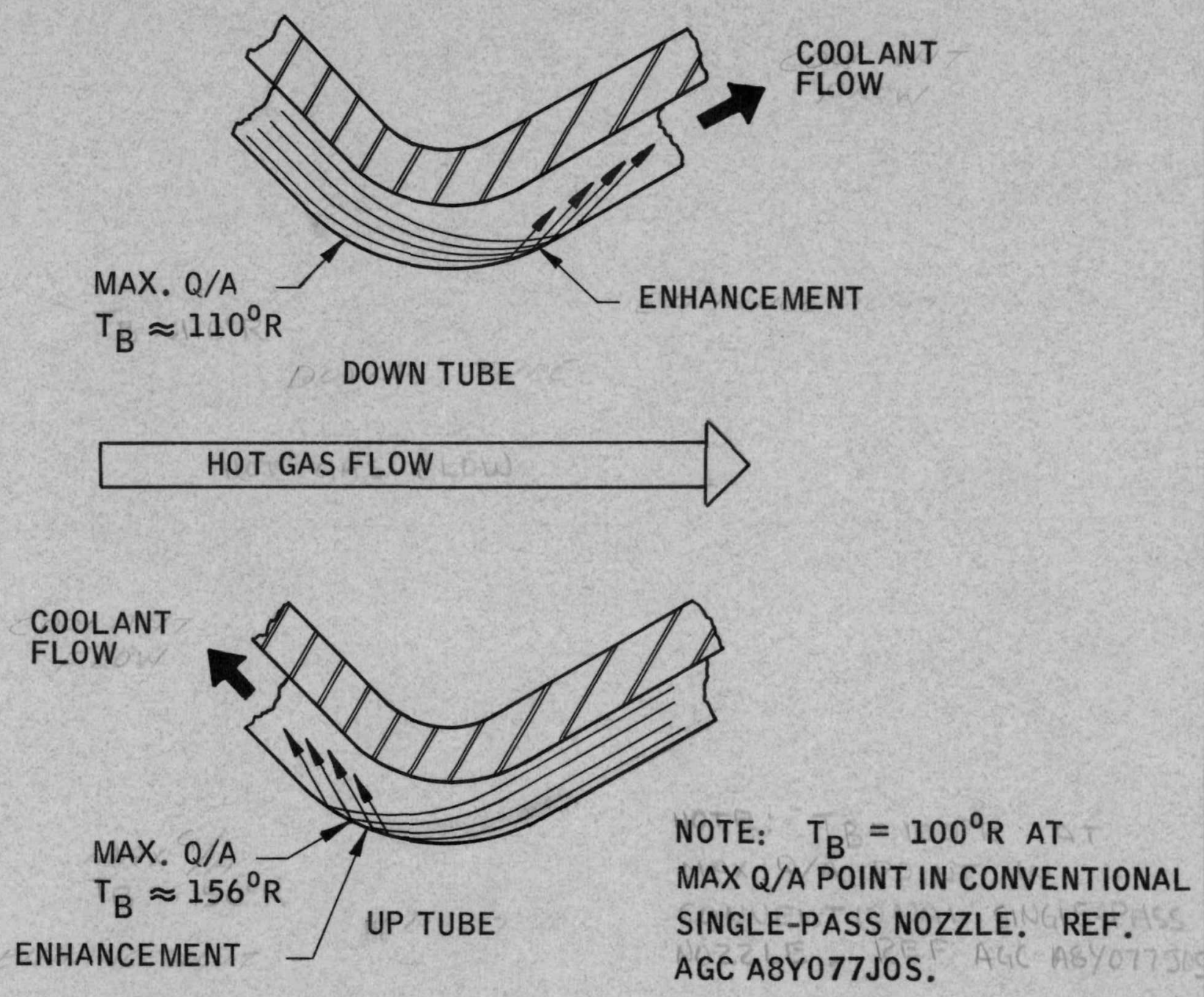
PinRo

NOZZIE COOIANT MACH NUMBE

VS. COOIAINT IIIET \& DISCHARGE TRIPERAIURE

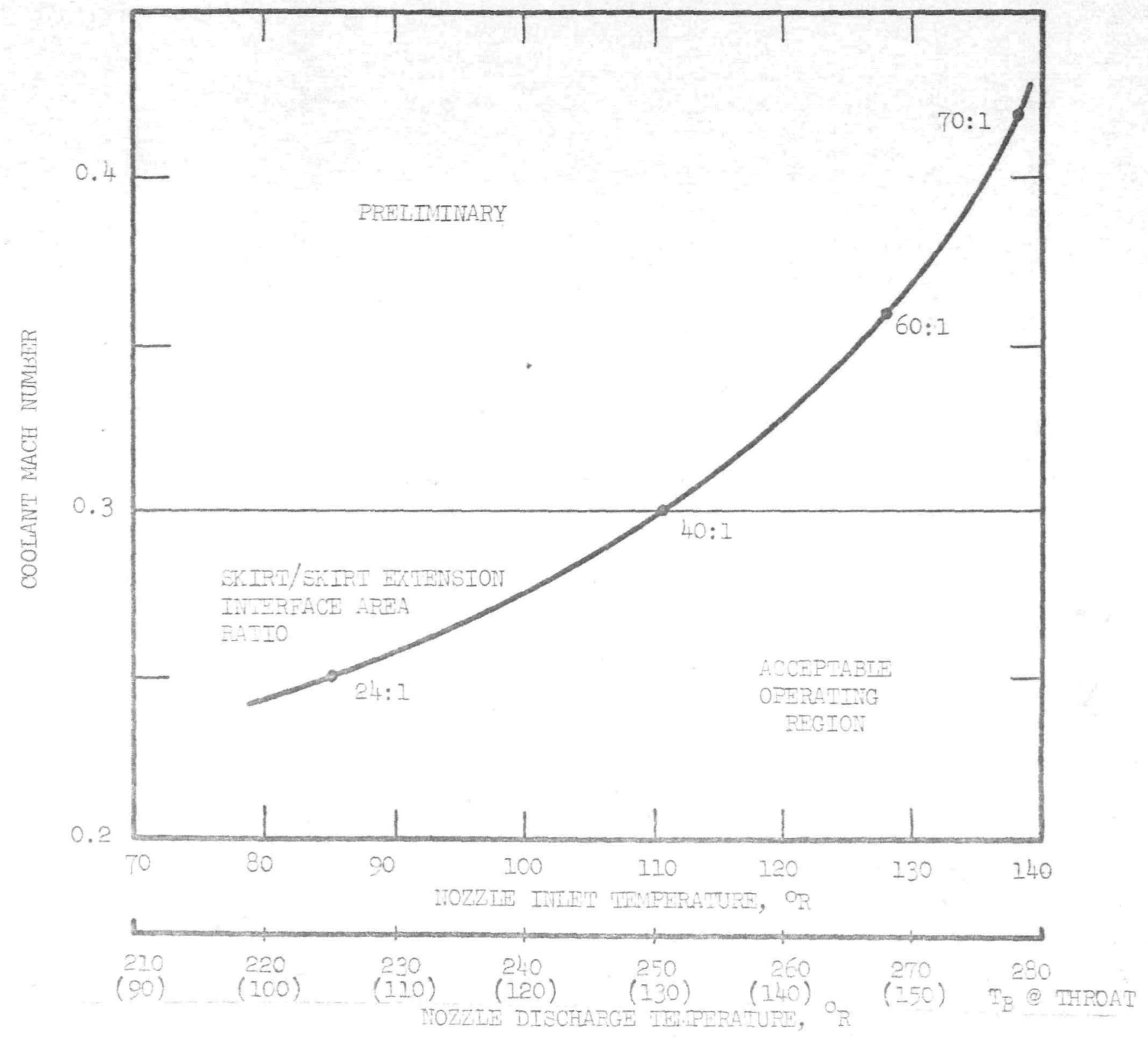




\section{0}

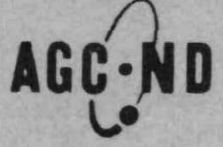

PARAMETRIC COMPARISON - FULL FLOW ENGINE TO HOT BLEED ENGINE SKIRT

\section{COMPONENT/PARAMETER}
(A) DESIGN
ABOUT THE SAME
(B) FABRICATION
NO CHANGE
(C) PERFORMANCE
NO CHANGE
(D) RELIABILITY
NO CHANGE
(E) WEIGHT
SLIGHTLY HEAVIER
(F) LENGTH
LONGER FOR THE SAME PERFORMANCE *
(G) COST
NO CHANGE
(H) MAINTAINABILITY
NO CHANGE
(I) OVERALL EVALUATION
NO CHANGE
* REPLACES TRUNCATED 100:1 SEGMENT 


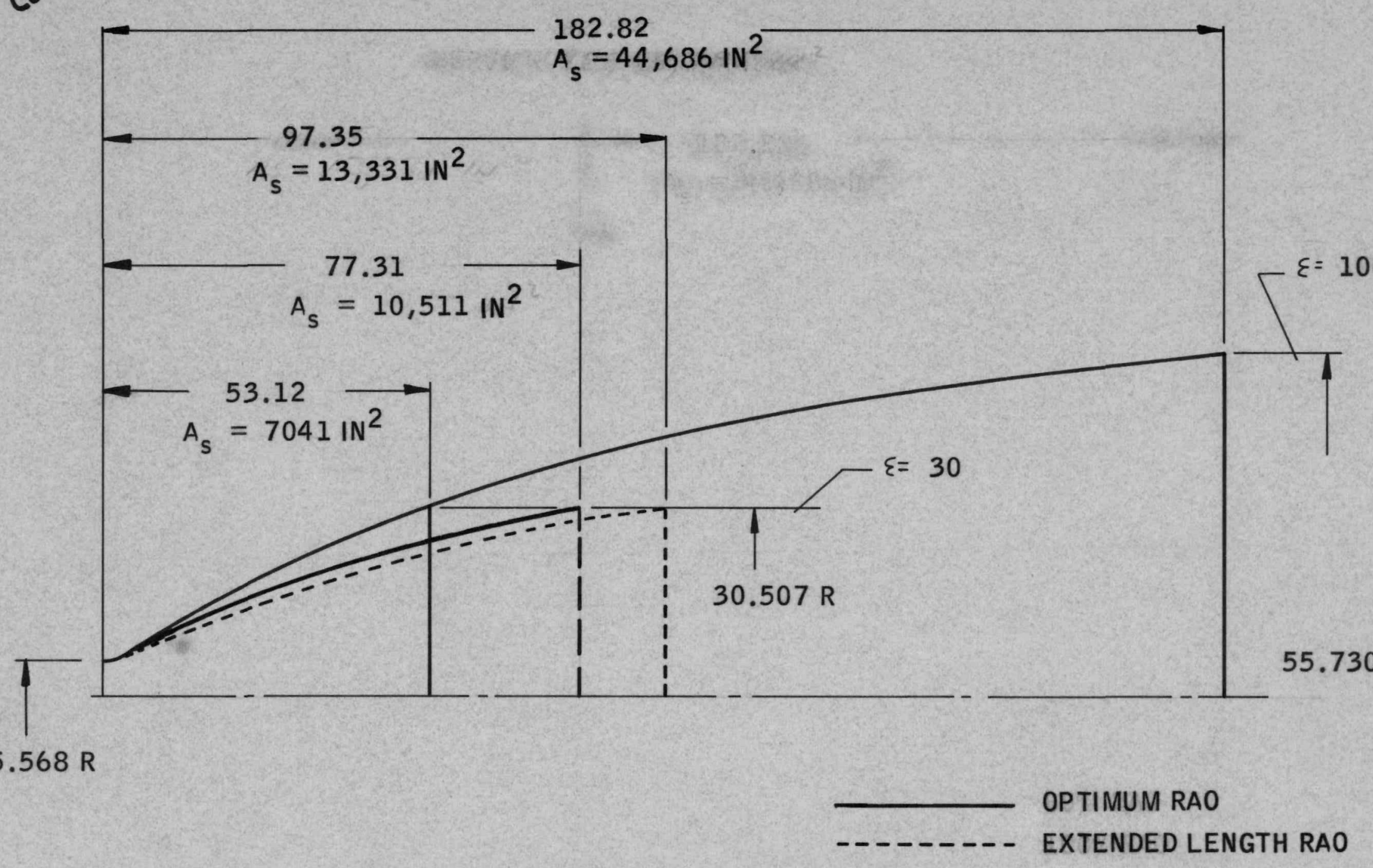




\section{AGcînD}

PARAMETRIC COMPARISON - FULL FLOW ENGINE TO HOT BLEED ENGINE

\section{SKIRT EXTENSION}

COMPONENT/PARAMETER
(A) DESIGN
GRAPHITE N/C CRYO-COOLED N/C TURBINE GAS-COOLED DELETE
(B) FABRICATION
$\mathrm{N} / \mathrm{C}$
(C) PERFORMANCE
$\mathrm{N} / \mathrm{C}$
(D) RELIABILITY
$\mathrm{N} / \mathrm{C}$
(E) WEIGHT
$\mathrm{N} / \mathrm{C}$
(F) LENGTH
$\mathrm{N} / \mathrm{C}$
(G) $\operatorname{COST}$
$\mathrm{N} / \mathrm{C}$
(H) MAINTAINABILITY
$\mathrm{N} / \mathrm{C}$
(1). OVERALL EVALUATION
SKIRT EXTENSION NOT NEEDED WITH FULL FLOW ENGINE TO MEET I SP 825 SECS 


\section{A. G. CARB - IOI}
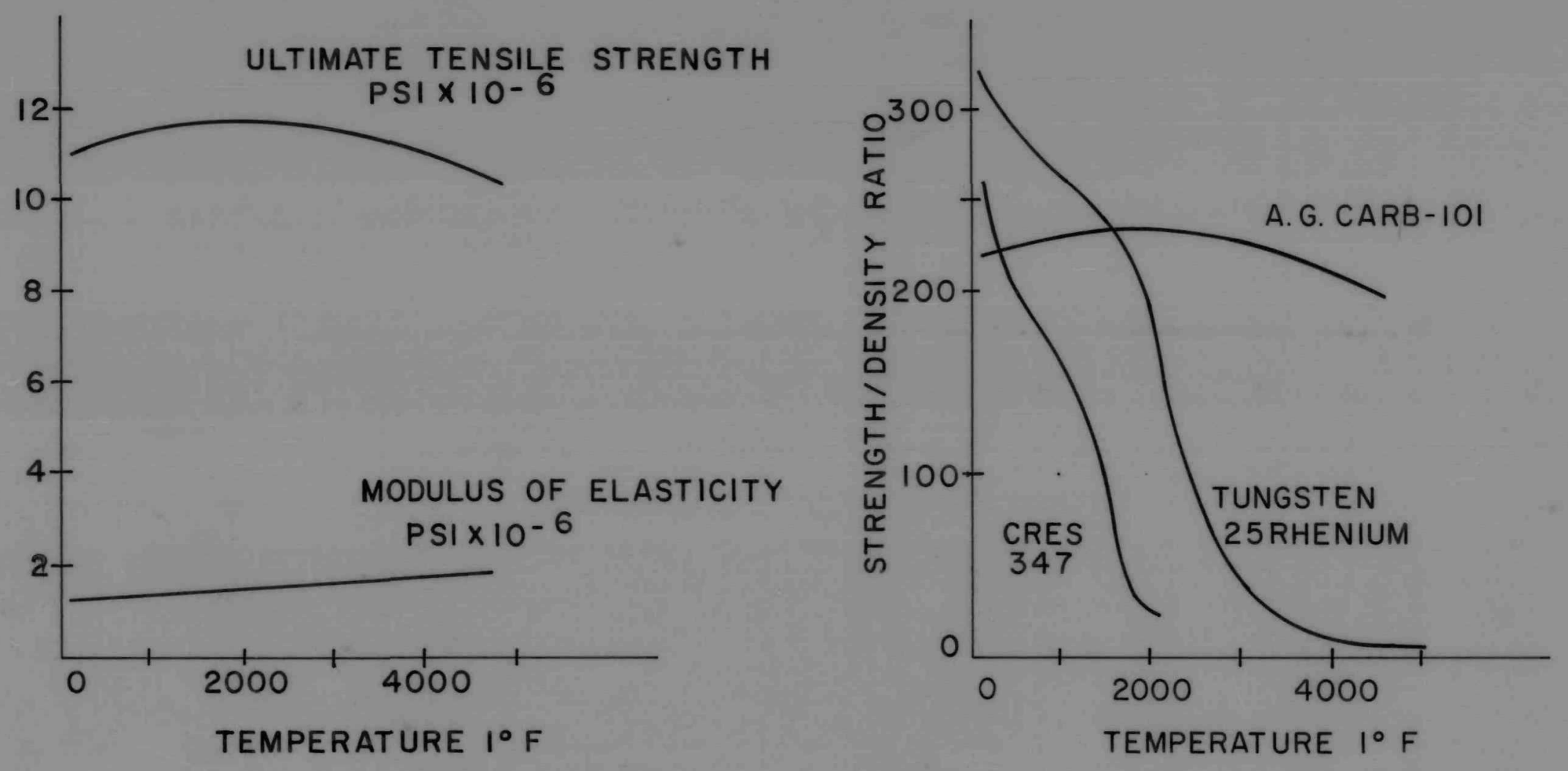


\section{A. G. CARB - 101}
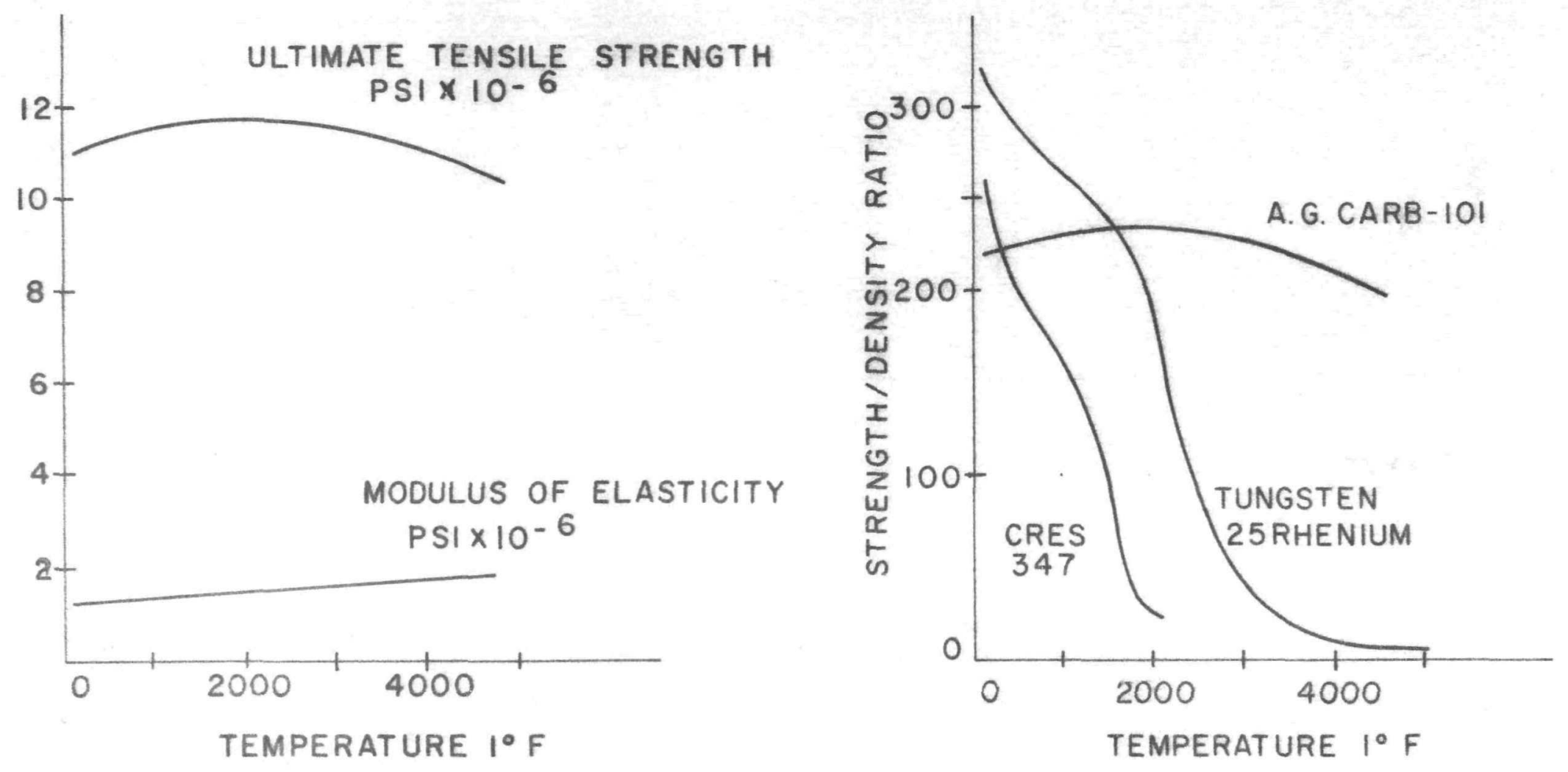


\section{LINES}

COMPONENT/PARAMETER
(A) DESIGN
SAME
(B) FABRICATION
SIMPLIFIED BECAUSE LINE LENGTH REDUCED
(C) PERFORMANCE
UNKNOWN
(D) RELIABILITY
HIGHER DUE TO ELIMINATION OF HOT GAS LINES AND REMOVAL OF OTHERS FROM HIGH RADIATION FLUX ZONES
(E) WEIGHT
1/3 TO 1/2 LIGHTER HT BLD - 1220*, F.F. W/STD PDL - 800\#, F.F. W/PART PDL $-600 \#$, F.F. W/PV/DBL
(F) LENGTH
SHORTER
(G) $\cos T$
SAME
(H) MAINTAINABILITY
NO CHANGE
(1) DEVELOPMENT TESTING
SAME
(J) OVERALL EVALUATION 







\section{AGC.:ND}

\section{EQUATIONS GOVERNING HEAT TRANSFER}

RATE IN HOT BLEED PORT INSERTS

COOLANT SIDE

$$
\begin{aligned}
N_{u_{f}}= & 0.0208\left(\frac{P_{f} v_{0} D_{h}}{\mu_{f}}\right)^{0.8} P_{r_{f}}\left(1+0.01457 \frac{U_{w}}{U_{b}}\right) \\
& \text { PRIMARY EFFECT }=\left(\frac{v_{0}}{2}\right)^{0.8} \\
& \text { OF REDUCING } \\
& \text { FLOW } 50 \%
\end{aligned}
$$

HOT GAS SIDE

$$
\begin{array}{ll}
N_{u_{f}}=0.5225 & \left(\frac{\rho v_{0} D_{b}}{\mu_{f}}\right)^{0.6} \\
\text { WHERE: } & \rho v_{0}=\left[\left(\frac{W}{A}\right)_{\text {NOZZLE }}^{2}+\left(\frac{W}{A}\right)_{\text {HOT BLEED }}^{2}\right]^{1 / 2} \\
& \left(\frac{W}{A}\right)_{\text {HOT BLEED }}^{2} \text { SMALL COMPARED WITH }\left(\frac{W}{A}\right)_{\text {NOZZLE }}^{2}
\end{array}
$$




\section{DUAL BLEED PORT INSERTS}

DESIGN \# 1 - DESIGN FOR OPTIMUM OPERATION AT 100\% DILUENT AND HOT GAS FLOW EACH

\section{DISADVANTAGES}

1. INSERT AND TIL UNDERCOOLED AT $50 \%$ DILUENT FLOW AVAILABILITY

2. DILUENT CONTROL VALVE REQUIRED FOR EACH DILUENT LINE

3. IF $100 \%$ OF FLOW WERE TAKEN THROUGH ONE INSERT, SOME DILUENT FLOW WOULD BE REQUIRED THROUGH INOPERATING BRANCH, THEREBY RESULTING IN PERFORMANCE LOSS

4. INCREASE PROGRAM COST

\section{ADVANTAGES}

NONE

\section{CONCLUSION}

LESS RELIABLE SYSTEM THAN ONE BLEED PORT INSERT BECAUSE OF THESE THREE DISADVANTAGES 
SINGLE VS DUAL BLEED PORT INSERT ASSE MBLIES

DUAL BLEED PORT INSERTS (cont)

DESIGN \# 2 - DESIGN FOR OPTIMUM OPERATION AT 50\% DILUENT AND HOT GAS FLOW DISADVANTAGES

1. DILUENT CONTROL VALVES REQUIRED

2. IF $100 \%$ OF FLOW WERE REQUIRED THROUGH ONE CIRCUIT, SOME DILUENT FLOW WOULD BE REQUIRED THROUGH INOPERATIVE BRANCH RESULTING IN:

a. DIFFICULTY IN OBTAINING $100 \%$ FLOW THROUGH ON BRANCH WITH EXISTING IMPEDANCES AND AVAILABLE SYSTEM $\triangle P$.

b. LOSS OF PERFORMANCE DUE TO LOSS OF DILUENT IN INOPERATIVE BRANCH

3. DESIGN AND DEVELOPMENT TASKS WOULD BE MORE DIFFICULT WITH $50 \%$ DILUENT AVAILABILITY

4. PROGRAM COST INCREASED

\section{ADVANTAGES}

1. INCREASED INSERT RELIABILITY IN OPERATIVE BRANCH AT $100 \%$ DILUENT FLOW, ALTHOUGH INSERT RELIABILITY IN INOPERATIVE BRANCH WOULD BE LESS 
DUAL BLEED PORT INSERTS (Cont)

DESIGN \# 3 - DESIGN A BLEED PORT INSERT WHICH IS UNSENSITIVE TO DILUENT FLOW

DISADVANTAGES

1. AVAILABLE MATERIALS FOR UNCOOLED INSERT DESIGN ARE LIMITED TO REFRACTORY METALS OR GRAPHITE. THESE MATERIALS OFFER ONE OR MORE OF THE FOLLOWING DISADVANTAGES:

a. BRITTLE AT LOW TEMPERATURES

b. SUSCEPTIBLE TO CHEMICAL ATTACK IN HOT HYDROGEN AND/OR OXYGEN

c. CHANGE IN MECHANICAL PROPERTIES DURING THERMAL CYCLING

2. DEVELOPMENT TIME AND COST WOULD BE GREATER THAN CONVECTIVELY COOLED DESIGN

3. EROSION OF UNCOLLED INSERT COULD RESULT IN TIL OR TURBINE DAMAGE

4. PROGRAM COST INCREASED

\section{ADVANTAGES}

1. WOULD ALLOW INDEPENDENT OPERATION OF BLEED PORT INSERTS WITHOUT SACRIFICING RELIABILITY 
DUAL BLEED PORT INSERTS (cont)

DESIGN \# 4 - DESIGN FOR 100\% FLOW AND OPERATE ONE AT A TIME

DISADVANTAGES

1. LOSS OF PERFORMANCE DUE TO THE NECESSITY OF MAINTAINING MINIMUM DILUENT FLOW THROUGH STANDBY CIRCUIT

2. INCREASED PROGRAM COST

3. SWITCHING OPERATION TO CHANGE FROM ONE CIRCUIT TO ANOTHER WOULD CAUSE DIFFICULTIES

ADVANTAGES

1. COMPONENTS DOWNSTREAM OF BLEED PORT INSERT WOULD BE SUBJECTED TO LESS THERMAL CYCLING 


\section{AGc. HD}

SINGLE VS DUAL BLEED PORT INSERT ASSEMBLIES

SINGLE BLEED PORT INSERT - DESIGN WITH TIL BRANCHING OUT TO DUAL TPCV'S AND TPA'S

1. HAS NONE OF THE DISADVANTAGES LISTED FOR DUAL BLEED PORT INSERTS

2. MAXIMUM RELIABILITY MAY BE DESIGNED INTO SINGLE BLEED PORT INSERT WITHOUT THE COMPROMISES ASSOCIATED WITH DUAL INSERT DESIGN

3. TECHNOLOGY EXPERIENCE INDICATES PRIMARY MODE OF INSERT FAILURE IS HAIRLINE LINER CRACKING. PENETRATING SELF FILM COOLING IS GENERATED WHICH PREVENTS CATASTROPHIC FAILURE 


\section{ac.No}

HOT BLEED PORT CHEMICAL

SIMULATION TESTS ON S/N O2T NOZZLE

\begin{tabular}{|c|c|c|c|c|c|c|c|c|c|c|}
\hline \multirow{2}{*}{$\begin{array}{l}\text { TTEST } \\
\text { NO. }\end{array}$} & \multirow{2}{*}{$\begin{array}{l}\text { DUR. } \\
\text { (SEC.) } \\
\end{array}$} & \multirow{2}{*}{$\begin{array}{c}P_{c} \\
(P S I A) \\
\end{array}$} & \multirow[b]{2}{*}{$\underline{M R}$} & \multirow{2}{*}{$\begin{array}{l}\dot{\mathrm{W}}_{\mathrm{H} . \mathrm{G}} \\
(\mathrm{LB} / \mathrm{SEC}) \\
\end{array}$} & \multirow{2}{*}{$\begin{array}{l}\text { DIL } \\
\text { (LB/SEC, } \\
\text { EXCLUDING } \\
\text { BYPASS) } \\
\end{array}$} & \multirow{2}{*}{$\begin{array}{r}\% \text { DIL } \\
\text { BYPASS } \\
\end{array}$} & \multirow{2}{*}{$\begin{array}{r}\text { DII } \\
P \\
\text { (PSI) } \\
\end{array}$} & \multirow{2}{*}{$\begin{array}{l}\text { TURBINE } \\
\text { INLETT } \\
\text { TE:MP }\left({ }^{\circ}\right) \\
\end{array}$} & \multicolumn{2}{|c|}{$\begin{array}{l}\text { PREDICIED BLEED PORT LINUR } \\
\left.\text { WALI TEMPERATURE ( }{ }^{\mathrm{R}} \mathrm{R}\right)\end{array}$} \\
\hline & & & & & & & & & OVER SPACERS & BETWEEN SPACERS \\
\hline 001 & 15.07 & 679 & 4. 71 & 6.44 & $3.47 *$ & 8.3 & 54 & 1590 & 2232 & 2047 \\
\hline $002 * *$ & 1.7 & - & - & - - & - & 8.3 & - & - & - & - \\
\hline 003 & 6.5 & 721 & 3.6 & $-* *$ & 5.38 & 8.3 & 71 & 903 & - & - \\
\hline $004 * *$ & 3.5 & - & - & - & - & 8.3 & - & - & - & - \\
\hline 005 & 5.8 & 645 & 5.0 & $-* *$ & $7.1 * * *$ & 8.3 & 15 & 650 & - & - \\
\hline 006 & 20.8 & 736 & 4.85 & 5.51 & 5.72 & 8.3 & 60 & 1250 & 2053 & 1790 \\
\hline 007 & 20.5 & 789 & 4.66 & 5.64 & 5.65 & 8.3 & 52 & 1270 & 2053 & 1792 \\
\hline 008 & 20.8 & 801 & 4.63 & 4.76 & 6.02 & 8.3 & 60 & 1280 & 1970 & 1709 \\
\hline $009 * *$ & 1.3 & - & - & - & - & 8.3 & - & - & - & - \\
\hline 010 & 20.4 & 801 & 4.63 & 4.85 & 5.52 & & 62 & 1550 & 1997 & 1735 \\
\hline
\end{tabular}

*DILUENT FLOW WAS RESTRICTED BY ICE FORMING IN THE DILUH'NT MIXER UPSTREAM OF THE HOT BLFED PORT DILUENT INLET. **NO DATA DUE TO SHORT DURATION OF TEST.

***MAIN DILUENT VALVE DID NOT OPEN. 


\begin{tabular}{|c|c|c|c|c|c|c|c|c|c|c|}
\hline $\begin{array}{l}\text { TEST } \\
\text { NO. }\end{array}$ & $\begin{array}{l}\text { DUR. } \\
\text { (SEC.) }\end{array}$ & $\begin{array}{l}\text { Pc } \\
\text { (PSIA) }\end{array}$ & MR & $\dot{\mathrm{W}}_{\text {(LB/SEC) }}$ & $\begin{array}{l}\text { UB/SEC, } \\
\text { EXCLUDIG } \\
\text { BYPASS) }\end{array}$ & $\begin{array}{l}\% \text { DLL } \\
\text { BYPASS }\end{array}$ & $\begin{array}{l}\mathrm{DIL} \\
\triangle P \\
(\mathrm{PSI})\end{array}$ & $\begin{array}{l}\text { TURBWE } \\
\text { TELET }\end{array}$ & \multicolumn{2}{|c|}{$\begin{array}{l}\text { PREPICTED BLEED PORT UNER } \\
\text { WALL TENPERATURE ( } R \text { R) } \\
\text { OVER SPACERS } \\
\text { BETWEEN SPACERS }\end{array}$} \\
\hline 001 & 15.07 & 679 & 4.71 & 6.44 & $3.47 *$ & 8.3 & 54 & 1590 & 1232 & 2047 \\
\hline $002 *$ & 1.7 & - & - & - & - & 8.3 & - & - & - & - \\
\hline 003 & 6.5 & 721 & 3.6 & $-t+k$ & 5.38 & 8.3 & 71 & 903 & - & - \\
\hline 004 *t & 3.5 & - & - & - & - & 8.3 & - & - & - & - \\
\hline 005 & 5.8 & 645 & 5.0 & $-*$ & $7.1^{* *}$ & 8.3 & 15 & 650 & - & - \\
\hline 006 & 20.8 & 736 & 4.85 & 5.51 & 5.72 & 8.3 & 60 & 1250 & 2053 & 1790 \\
\hline 007 & 20.5 & 789 & 4.66 & 5.64 & 5.65 & 8.3 & 52 & 1270 & 2053 & 1792 \\
\hline 008 & 20.8 & 801 & 4.63 & 4.76 & 6.02 & 8,3 & 60 & 1280 & 1970 & 1709 \\
\hline 009 * & 1.3 & $-\infty$ & - & - & - & 8.3 & - & - & - & - \\
\hline 010 & 20.4 & 801 & 4.63 & 4.85 & 5.52 & 8.3 & 62 & 1550 & 1997 & 1735 \\
\hline
\end{tabular}

*DILUENT FLOW WAS RESTRICTED BY ICE FORMING IN THE DILUENT MIXER UPSTREAM OF THE HOT BLEEDPORT DILUENT INLET * NO DATA DUE TO SHORT DURATION OF TEST

**MAIN DILUENT VALVE DID NOT OPEN 


\section{NRX - EST TESTS}

TEST

RUN

NO.

$P_{C}$

(PSIA)

${ }^{\mathrm{T}} \mathrm{C}$

EP 118

1

275

2500

220

2100

EP IIC

1

180

2000

230

2600

EP III

1

560

4200

580

3600

EP $N_{*}$

2

548

4000

EP IVA

2

560

4100

TOTAL MO. OF STARTS $=10$

$* \dot{W}$ TURB. $=6.5 \mathrm{LB} / \mathrm{SEC}, \dot{W}$ DIL. $=4.7 \mathrm{LB} / \mathrm{SEC}, \mathrm{PRESS}$. TURB. INLET $=384$ PSIA, TEMP. TURB. INLET $=114 \mathrm{I}{ }^{\circ} \mathrm{R}$.

PREDICTED MAXIMUM BLEED PORT LINER WALL TEMP. = $1921 \pm 50^{\circ} \mathrm{R}$ OVER SPACERS AND $1723 \pm 50^{\circ} \mathrm{R}$ BETWEEN SPACERS. 


\begin{tabular}{|l|l|l|}
\hline \multicolumn{1}{|c|}{ PARAMETER } & HOT BLEED & \multicolumn{1}{c|}{ FULL FLOW } \\
DESIGN & DEMONSTRATED & LESS COMPLEX BECAUSE NO HOT BLEED PORT \\
\hline FABRICATION & DEMONSTRATED & LESS COMPLEX BECAUSE NO HOT BLEED PORT \\
\hline PERFORMANCE & REFERENCE & EQUAL FOR SAME AREA RATIO \\
\hline RELIABILITY & REFERENCE & HIGHER \\
\hline WEIGHT & REFERENCE & LOWER FOR SAME PERFORMANCE \\
\hline LENGTH & REFERENCE & SHORTER FOR SAME PERFORMANCE \\
\hline $\begin{array}{l}\text { COST, NOZZLE } \\
\text { HARDWARE }\end{array}$ & REFERENCE & LOWER \\
\hline MAINTAINABILITY & DEMON STRATED & BETTER \\
\hline $\begin{array}{l}\text { CHEM. SIMULATION } \\
\text { TESTING REQD }\end{array}$ & YES & *NO \\
\hline
\end{tabular}

* * CHEM. SIMULATION TESTING OF NOZZLE CURRENTLY SCHEDULED FOR PURPOSE OF TESTING BLEED PORT ONLY 


\begin{tabular}{|c|c|c|c|c|c|c|c|c|}
\hline \multirow{3}{*}{ DESCRAPTION } & \multicolumn{4}{|c|}{$\triangle$ WEIGHT } & \multicolumn{4}{|c|}{ * EQUNALENT I } \\
\hline & \multirow{2}{*}{$\begin{array}{l}\text { MOZZLE } \\
\text { RKART }\end{array}$} & \multirow[b]{2}{*}{ LIMES } & \multirow[b]{2}{*}{$\begin{array}{l}\text { PRESSURE } \\
\text { VESSEL }\end{array}$} & \multirow[b]{2}{*}{$\Sigma \Delta w$} & \multicolumn{3}{|c|}{ SOURCE } & \multirow{2}{*}{$\begin{array}{l}\text { EQUN. } \\
\sum \Delta I_{3}\end{array}$} \\
\hline & & & & & $\Delta w$ & $\begin{array}{l}\text { MTER } \\
\text { STAGE } \\
\text { SL. }\end{array}$ & $\begin{array}{l}\text { HOT BEEED } \\
\text { FRACTION }\end{array}$ & \\
\hline \multicolumn{9}{|l|}{ MOZZLE $A_{d} / A_{t}=100.1$} \\
\hline \multicolumn{9}{|l|}{ ATTAIMABLE $I_{s}=845.1$} \\
\hline A. MOT BLEED & REF. & REF. & REF. & REF. & REF. & REF. & -20.0 & -20.0 \\
\hline B. FULLLFLOWL $\epsilon=100 \mathrm{r} 2 \mathrm{X}$ & $\because$ & $\cdots, x_{x}=$ & $\Rightarrow$ & $-\because$ & . & $x_{3}=$ & : & a . $t$ \\
\hline 2. CAYOCOOLEO EXTI & REF. & -420 & +300 & -120 & +0.7 & REF. & 0 & +0.7 \\
\hline 2. GRAPHITE EXTM & -747 & -420 & +300 & -867 & +5.5 & REF. & $\mathbf{0}$ & +5.5 \\
\hline c. FULL FLOW $(\epsilon=30: 1)$ & & & & & & & & \\
\hline ATTAMUBEE $I_{3}=825.6$ & -1090 & -420 & +300 & -1210 & +7.7 & +3.9 & 0 & +11.6 \\
\hline
\end{tabular}

* $\triangle$ P OF 40 PSI, $\triangle W$ OF 158 LBS, \& $\triangle L$ OF 27 IMCHES = 1 SEC Is (EACHO 


\section{COST SAYIMGS FOR FULL FLOW CYCLE}

A. MOZZLE ASSEMBLY

COMPONENT DEVELOPMENT HOZZLE (1)

BLEED PORT INSERTS (23)

TEST ASSEMAY COIPONENTS, WCL TOOLING

"R" SKIRT (1)

CHEMHCAL SIMULATION TESTING

2 ENGIMEERUNG MAN YEARS

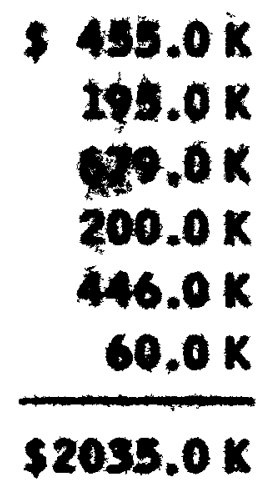

B. LIWES

TUROANE IMLET

PUIMP DISCHARGE

TUROUNE BSCHARCE

TOTAL MOZZLE ASSEMBLY

$\$ 2035.0 \mathrm{~K}$

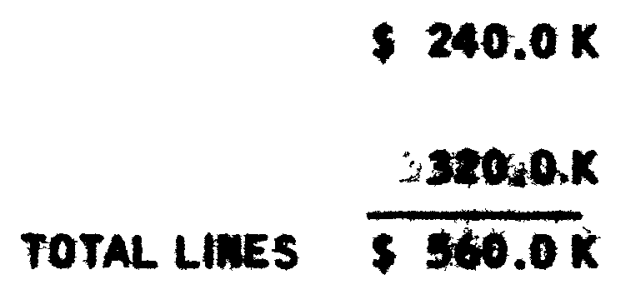

C. SKIRT ExTEUsion

(IF NOZZLE ASSY is 30:1) (19 UMITS + TOOLING

$\$ 427.0 \mathrm{~K}$

TOTAL SAMNGS \$7z22.0 K 


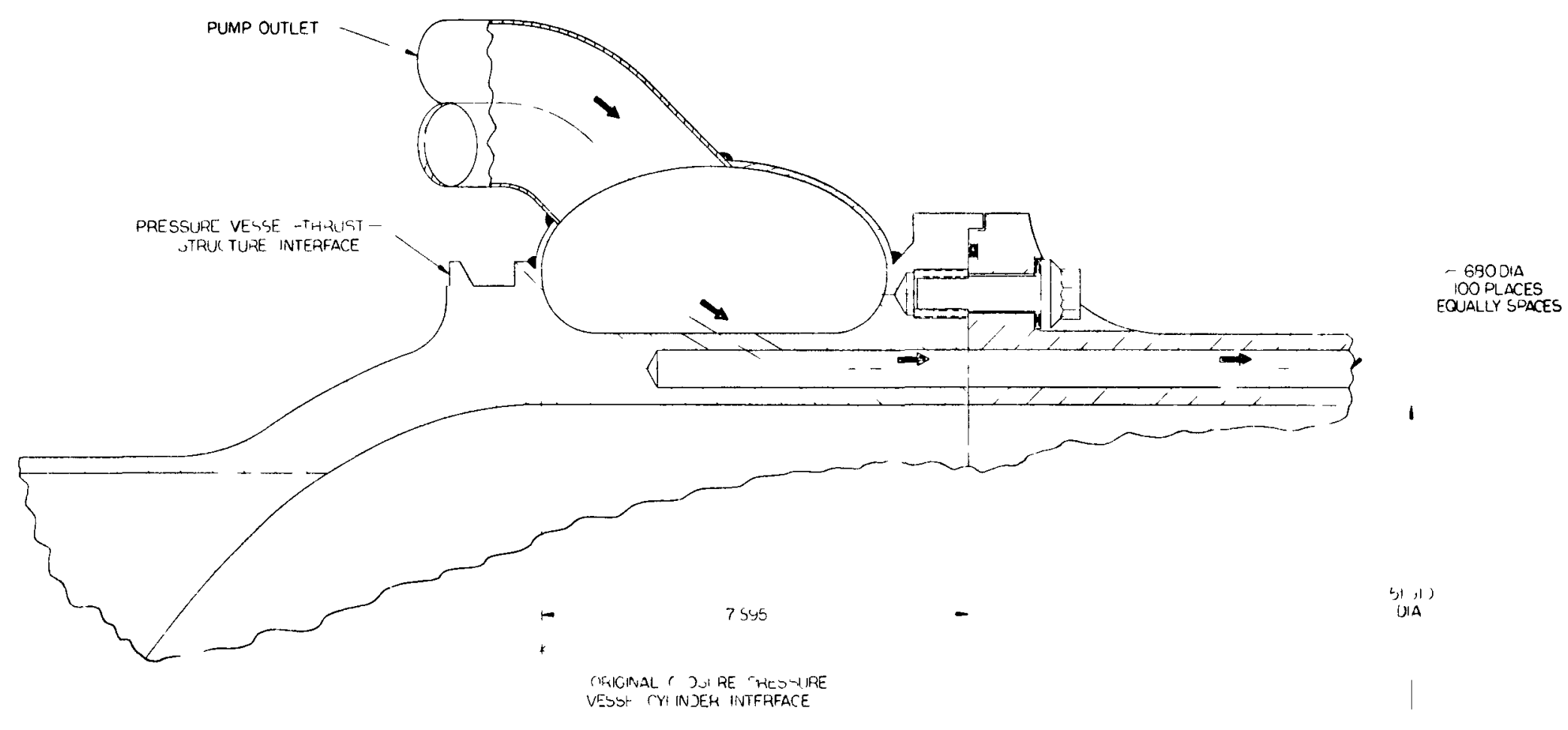

NERVA FULL FLOW ENGINE. PRESSURE VESSEL CLOSURE TO CYLINOER FLOW CONCEPT NO. 3 NOT USED IN PRESENTATION

WIEUGRIII: WIED IN REAP OF MASTER FILE SE:"101. 


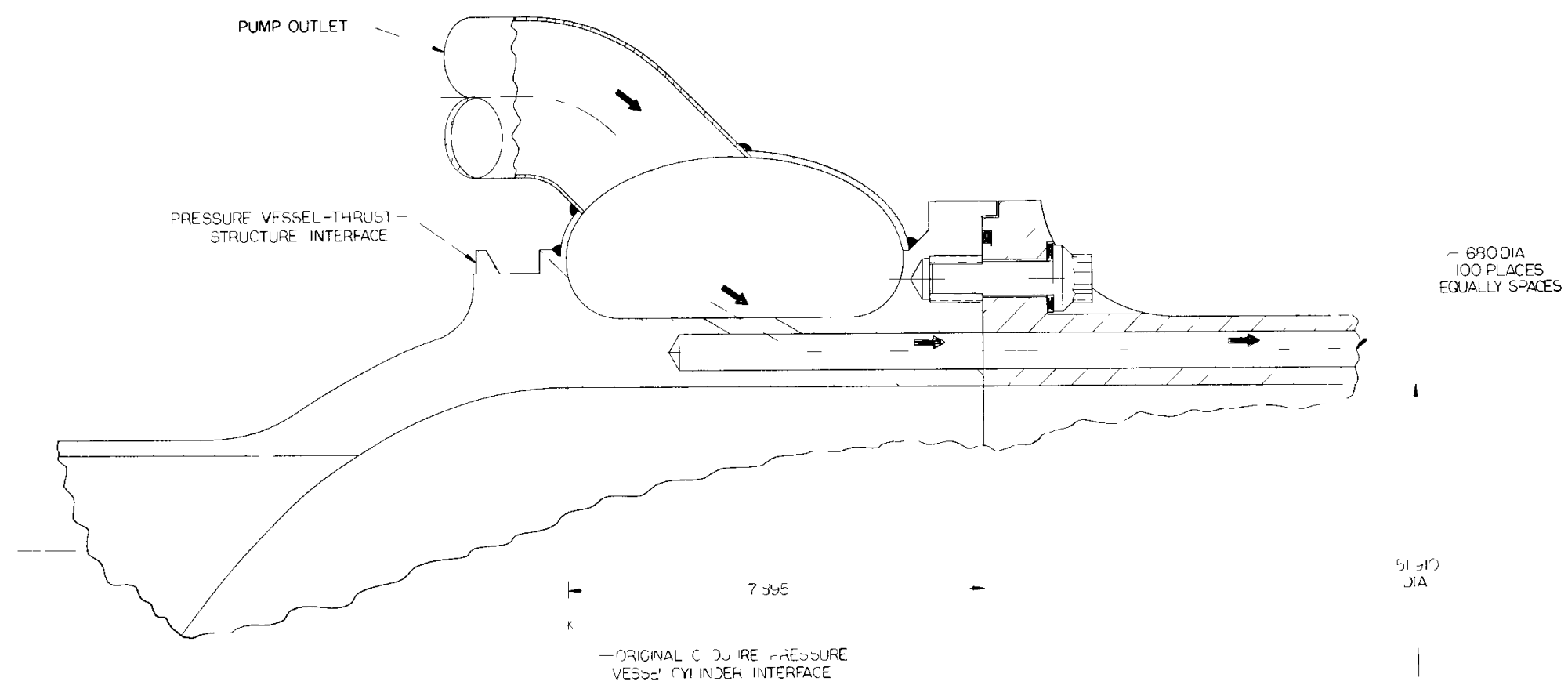




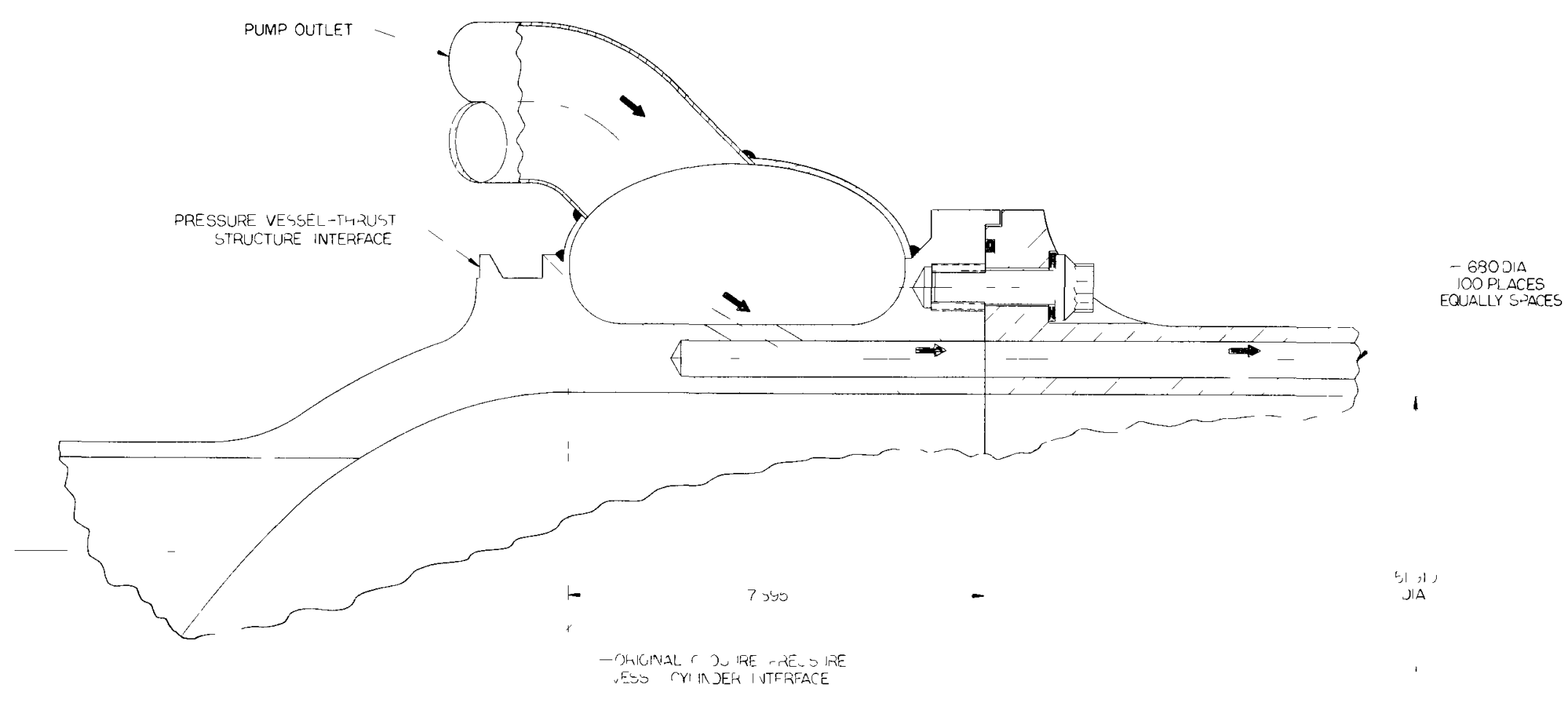




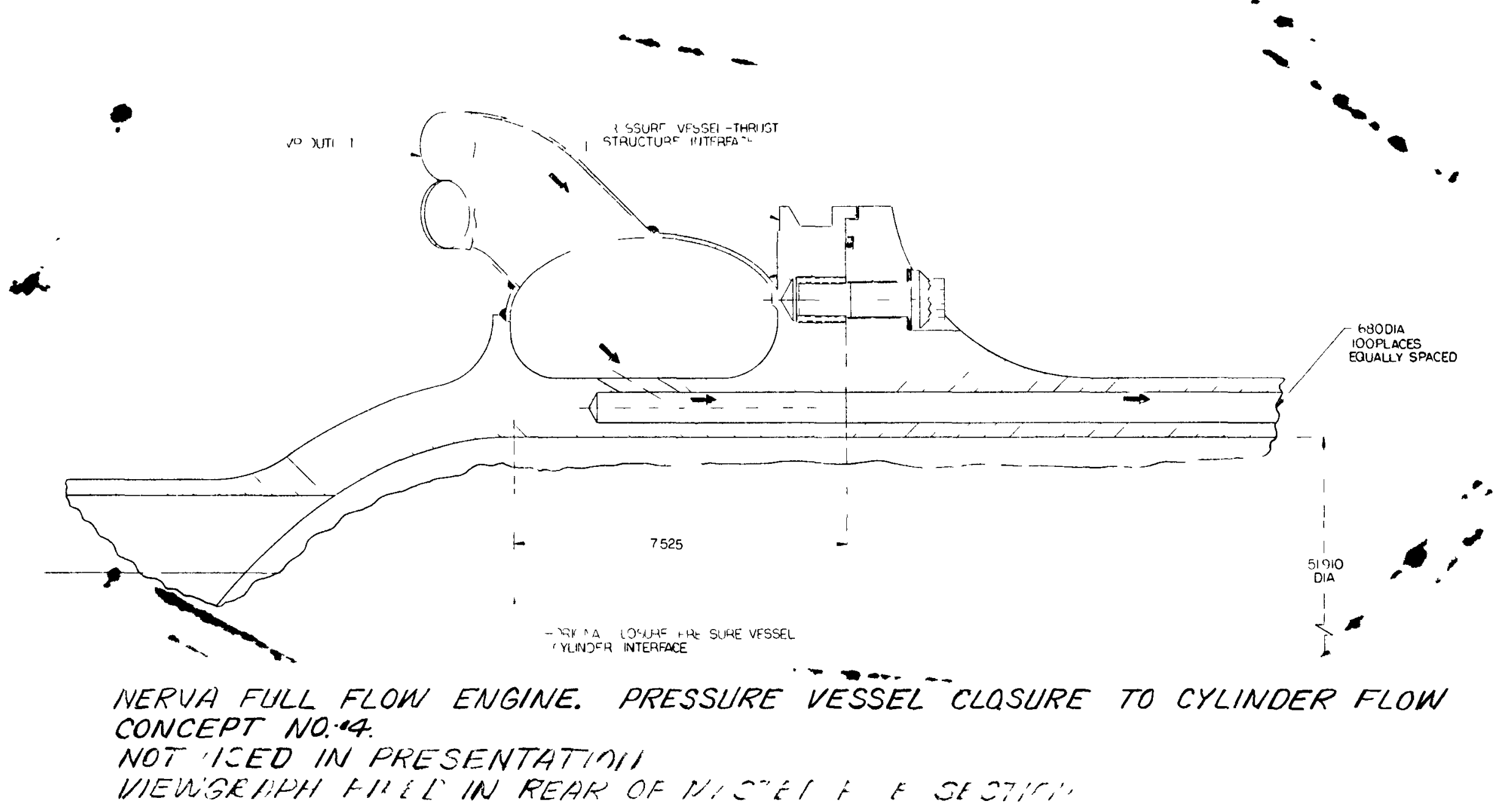




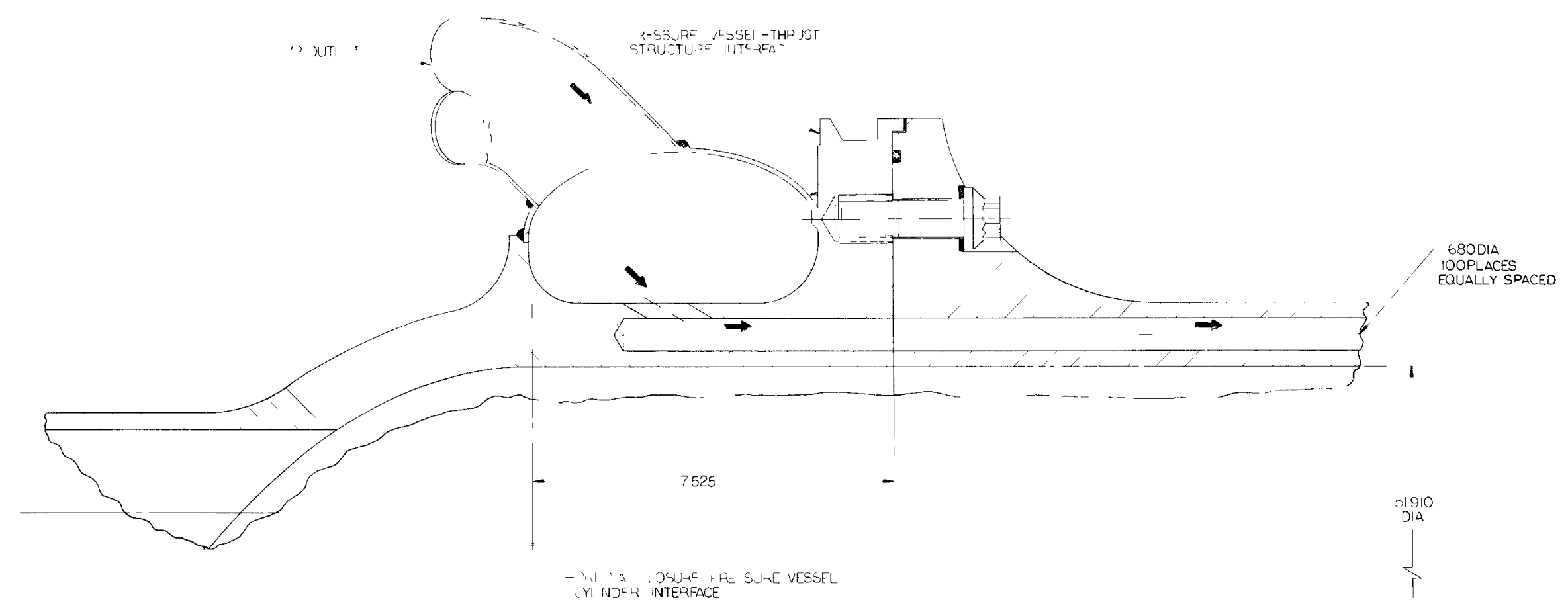




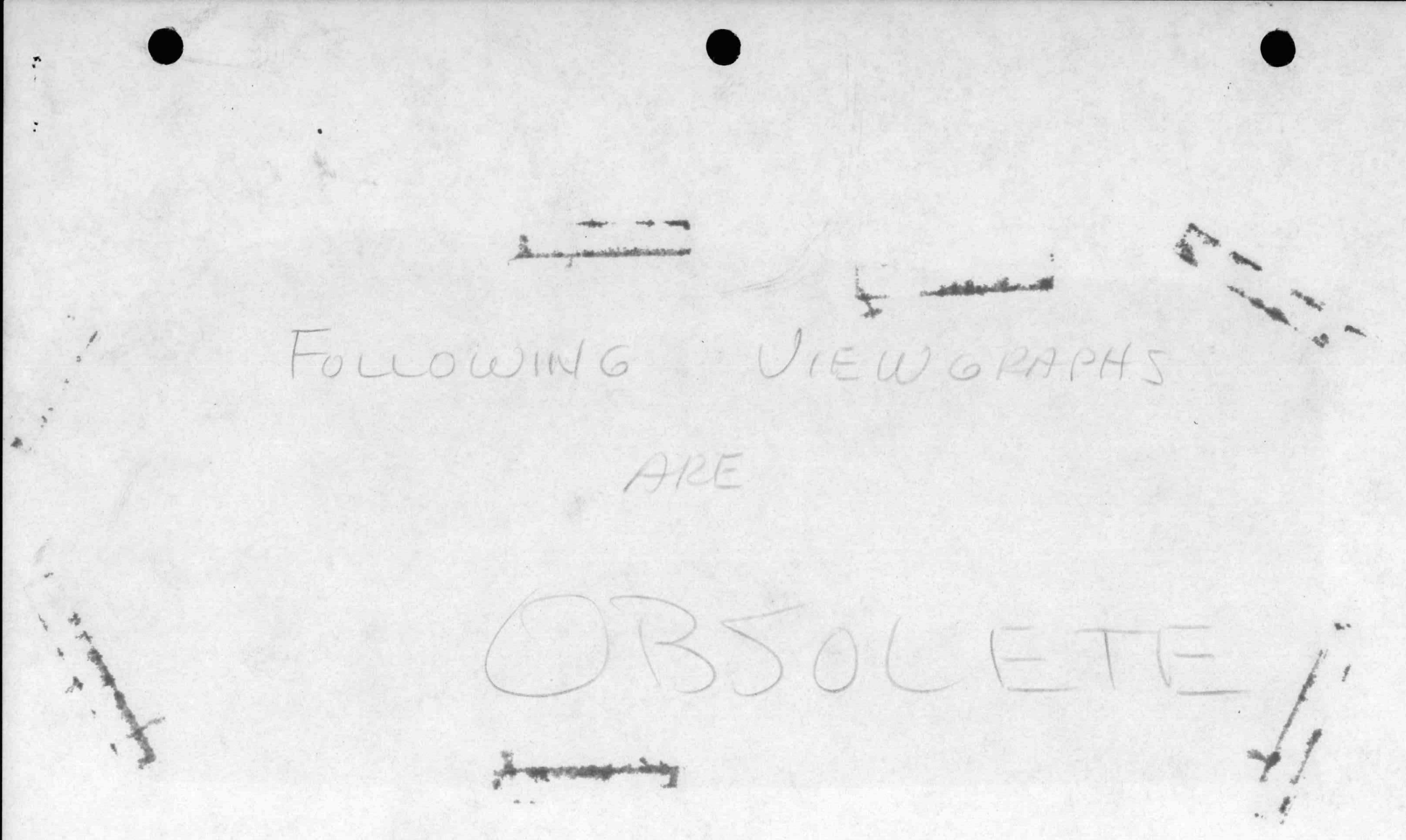


DUAL BLEED PORT INSERTS

DESIGN \#I - DESIGN FOR OPTIMUM OPERATION AT 100\% DILUENT AND HOT GAS FLOW EACH

\section{DISADVANTAGES}

1. INSERT AND TIL UNDER COOLED AT 50\% DILUENT FLOW AVAILABILITY

2. DILUENT CONTROL VALVE REQUIRED FOR EACH DILUENT LINE

3. IF $100 \%$ OF FLOW WERE TAKEN THROUGH ONE INSERT, SOME DILUENT FLOW WOULD BE REQUIRED THROUGH INOPERATING BRANCH, THEREBY RESULTING IN PERFORMANCE LOSS

4. INCREASE PROGRAM COST BY $\$ 500,000$

( $\$ 195,000$ ADDITIONAL BLEED PORT INSERTS \& $\$ 300,000$ ADDITIONAL NOZZLE MACHINING)

\section{ADVANTAGES}

NONE

\section{CONCLUSION}

LESS RELIABLE SYSTEM THAN ONE BLEED PORT INSERT BECAUSE OF THESE THREE DISADVANTAGES 
PARAMETRIC COMPARISON - FULL FLOW ENGINE TO HOT BLEED ENGINE

LINES

COMPONENT/PARAMETER
(A) DESIGN
REDUCED SCOPE BECAUSE HOT GAS LINES ARE ELIMINATED
(B) FABRICATION
SIMPLIFIED BECAUSE STANDARD MATERIALS CAN BE USED
(C) PERFORMANCE
UNKNOWN
(D) RELIABILITY
HIGHER DUE TO ELIMINATION OF HOT GAS LINES AND REMOVAL OF OTHERS FROM HIGH RADIATION FLUX ZONES
(E) WEIGHT
1/3 TO 1/2 LIGHTER HT BLD - 1220*, F.F.W/STD PDL - 800*, F.F. W/PART PDL - 600*, F.F.W/PV/DBL PASS NO. 2 - 500*
(F) LENGTH
SHORTER
(G) COST LESS
(H) MAINTAINABILITY NO CHANGE
(1) DEVELOPMENT TESTING REDUCED SCOPE DUE TO ELIMINATION OF HOT GAS LINES
(J) OVERALL EVALUATION SIGNIFICANTLY BETTER OVERALL BECAUSE OF ELIMINATION OF HOT GAS LINES 


\section{AGC.ND}

PARAMETRIC COMPARISON - FULL FLOW ENGINE TO HOT BLEED ENGINE SKIRT EXTENSION

COMPONENT/PARAMETER
(A) DESIGN
GRAPHITE N/A CRYO-COOLED N/A TURBINE GAS-COOLED DELETE
(B) FABRICATION
N/A
(C) PERFORMANCE
N/A
(D) RELIABILITY
N/A
(E) WEIGHT
N/A
(F) LENGTH
N/A
(G) $\operatorname{COST}$
N/A
(H) MAINTAINABILITY
N/A
(1) OVERALL EVALUATION
SKIRT EXTENSION NOT NEEDED WITH FULL FLOW ENGINE TO MEET I SP 825 SECS 


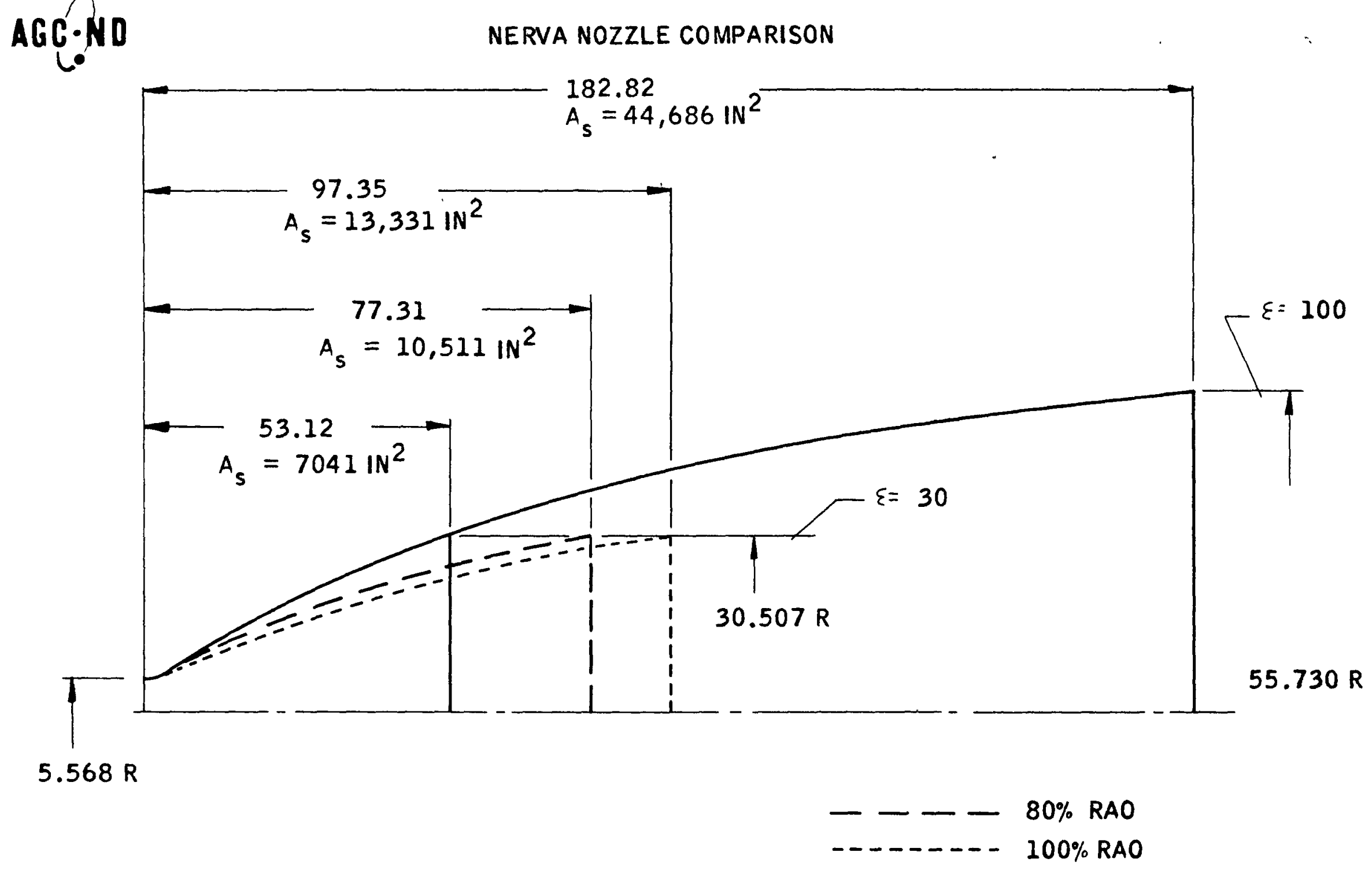




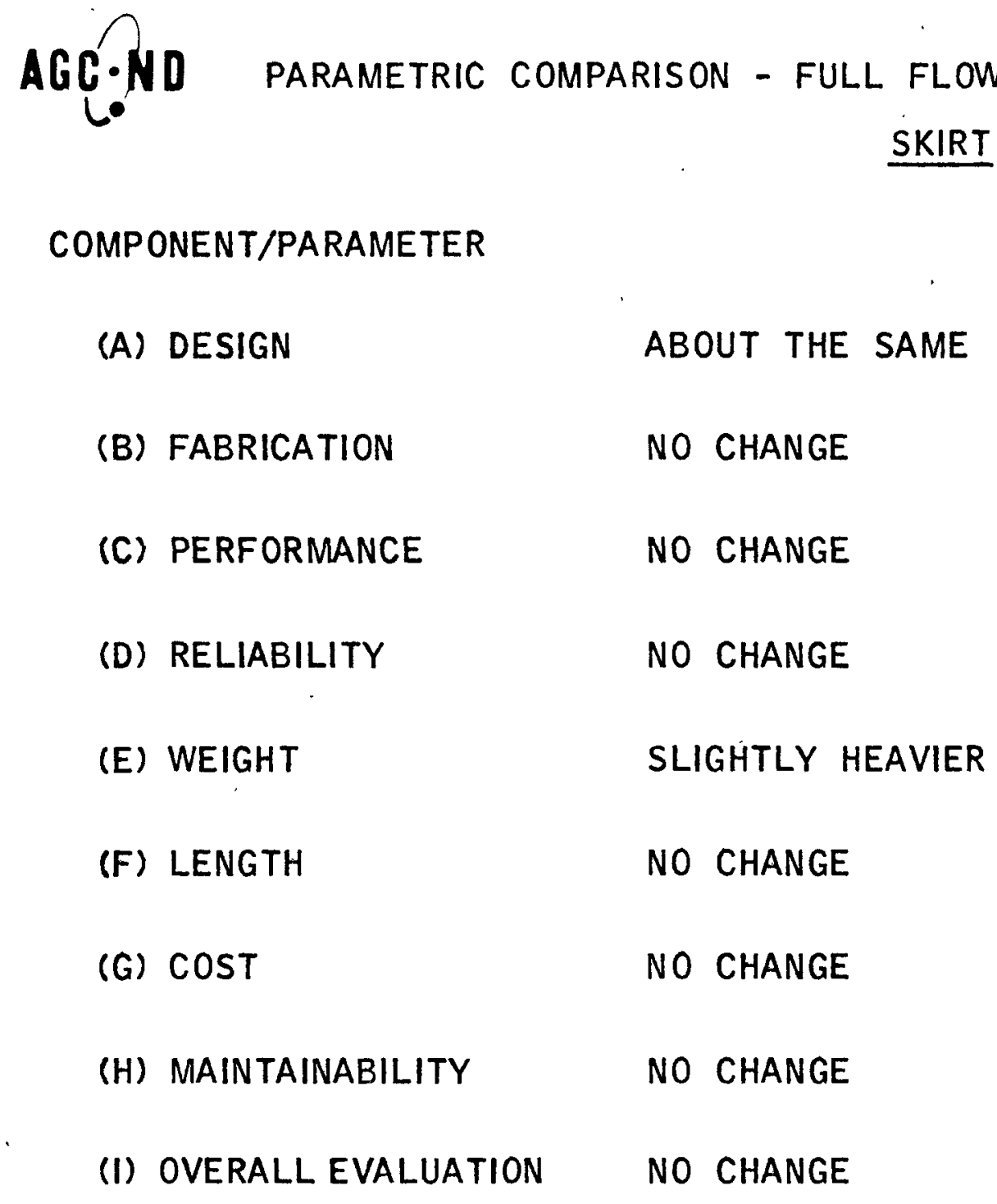



SHMLE VS DUML BLEED PORT IUSERT ASSEMBLIES

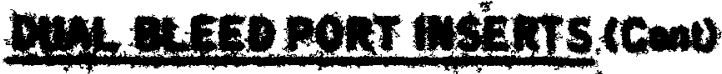

DEIEELE - BESIEI FOR OPTIMUM OPERATION AT 50\% DLLEET AMD HOT GAS FLOW

\section{ogsavuaruases}

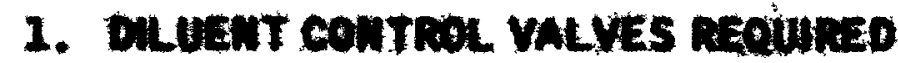

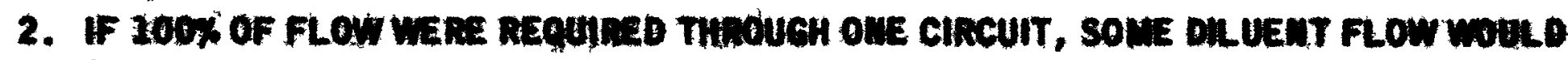

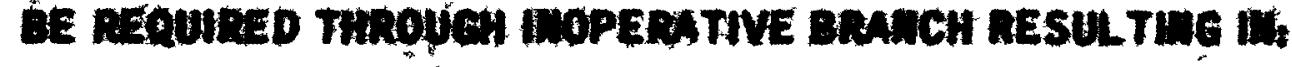

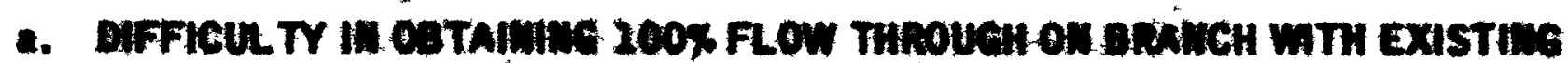
IIPEDMUES AMP AVALLALE SYSTEM $\triangle P$.

b. LOSS OF PERFORMAMCE DUE TO LOSS OF DLDEET II MOPERA TVE BRAMCH

3. OESIOW AND DEVELOPMENT TASKS WOULD EE MORE DHFFICULT WTH SO\% DLUENT AVAlLABHITY

4. PROGRAM COST IMCREASED BY $\$ 500,000$

\section{AOVGTHCES}

1. MEREASED IMSERT RELMARLITY MT ORERATIVE BRAMCH AT 100\% DR UEMT FLOW,

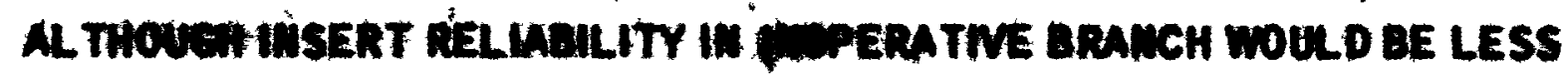


SIPLE VS OWAL HEED PÓRT IISERT ASSEMBLES

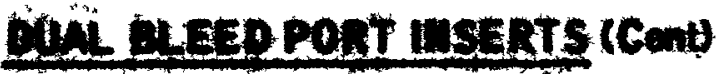

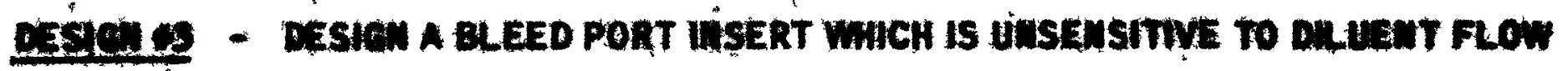

Benpantess

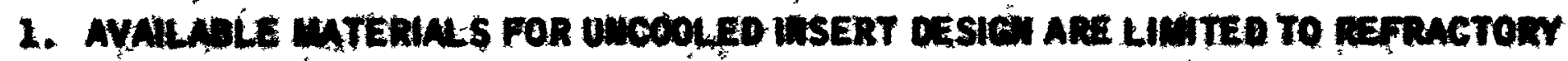

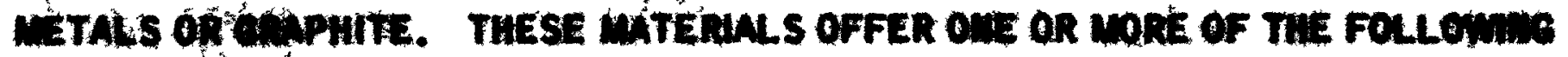
Msabuntaes.

A. BATTLE AT LOW TEIPERATURES

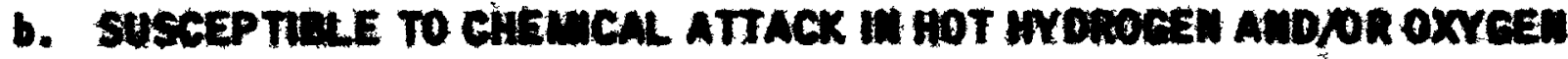

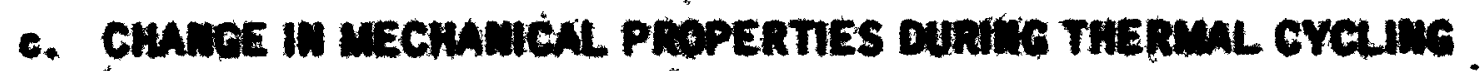

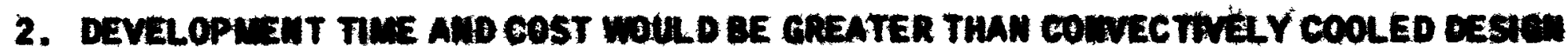

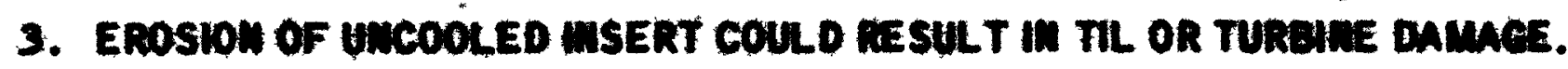

4. PROGMA COST IMCREASED in EXCESS OF $\$ 500,000$

\section{ABVAMTACES}

1. WOULD ALLOW IUDEPEUDENT OPERATION OF BLED PORT IISERTS WTHOUT SACAIFICING RELIABUITY 



\begin{tabular}{|l|l|l|}
\hline \multicolumn{1}{|c|}{ PARAMETER } & \multicolumn{1}{c|}{$\begin{array}{c}\text { HOT BLEED } \\
\text { DESIGN }\end{array}$} & FULL FLOW \\
\hline FABRICATION & PROVEN & LESS COMPLEX BECAUSE NO HOT BLEED PORT \\
\hline PERFORMANCE & REFERENCE & EQUAL FOR SAME AREA RATIO \\
\hline RELIABILITY & REFERENCE & HIGHER \\
\hline WEIGHT & REFERENCE & LOWER FOR SAME PERFORMANCE \\
\hline LENGTH & REFERENCE & SHORTER FOR SAME PERFORMANCE \\
\hline $\begin{array}{l}\text { COST, NOZZLE } \\
\text { HARDWARE }\end{array}$ & REFERENCE & LOWER \\
\hline MAINTAINABILITY & PROVEN & BETTER \\
\hline $\begin{array}{l}\text { CHEM. SIMULATION } \\
\text { TESTING REQD }\end{array}$ & YES & *NO FOR SAME PERFORMANCE \\
\hline
\end{tabular}

* 1. CHEM. SIMULATION TESTING OF NOZZLE CURRENTLY SCHEDULED FOR PURPOSE OF TESTING BLEED PORT ONLY

2. DEVELOPMENT TÉSTING OF SKIRT EXTENSION AT A $\mathrm{e}^{/ A_{t}}>30: 1$ CAN BE ACCOMPLISHED IN TULLAHOMA 


\begin{tabular}{|c|c|c|c|c|c|c|c|c|}
\hline \multirow[b]{2}{*}{ CONFIGURATION } & \multirow{2}{*}{$\begin{array}{l}\text { NOZZLE } \\
\& \\
\text { SKIRT }\end{array}$} & \multirow[b]{2}{*}{ LINES } & \multirow[b]{2}{*}{$\begin{array}{l}\text { PRESSURE } \\
\text { VESSEL }\end{array}$} & \multirow[b]{2}{*}{$\sum \Delta w$} & \multicolumn{4}{|c|}{ * EQUIVALENT SPECIFIC IMPULSE } \\
\hline & & & & & $\Delta \mathrm{W}$ & $\Delta \mathrm{L}$ & $\begin{array}{l}\text { HOT BLEED } \\
\text { FRACTION }\end{array}$ & $\sum \Delta l_{s}$ \\
\hline HOT BLEED $100: 1\left(I_{\mathrm{s}}=845.1\right)$ & REF. & REF. & REF. & REF. & REF. & REF. & -20.0 & -20.0 \\
\hline FULL FLOW $\quad 30: 1\left(l_{s}=825.6\right)$ & & & & & & & & \\
\hline A. DRILLED PRESS.VESSEL & -1213 & -620 & +1100 & -733 & +4.6 & +3.9 & 0 & +8.5 \\
\hline B. EXTERNAL LINES & -1213 & -420 & +300 & -1320 & +8.4 & +3.9 & 0 & +12.3 \\
\hline $\begin{array}{l}\text { FULL FLOW } 100: 1\left(I_{s}=845.1\right) \\
\text { A. DRILLED PRESS.VESSEL }\end{array}$ & & & & & & & & \\
\hline 1. CRYO COOLED SKIRT & REF. & -620 & +1100 & +480 & -3.0 & REF & 0 & -3.0 \\
\hline 2. GRAPHITE SKIRT & -747 & -620 & +1100 & -267 & +1.7 & REF & 0 & +1.7 \\
\hline B. EXTERNAL LINES & & & & & & & & \\
\hline 1. CRYO COOLED SKIRT & REF. & -420 & +300 & -120 & +0.7 & REF & 0 & +0.7 \\
\hline 2. GRAPHITE SKIRT & -747 & -420 & +300 & -847 & +5.4 & REF & 0 & +5.4 \\
\hline
\end{tabular}

$\triangle P$ OF 40 PSI, $\triangle W$ OF 158 LBS, OR $\triangle L$ OF 27 INCHES $=1$ SEC. 
A6c.jp

PARAMETRIC COMPARISON - FULL FLOW NOZZLE

CONCEPTS TO HOTESEO CYCLE NOZZLE

\begin{tabular}{|c|c|c|c|c|c|c|}
\hline \multirow[b]{2}{*}{ PARAMETER } & \multirow{2}{*}{$\begin{array}{l}\text { HOT } \\
\text { BLEED } \\
\text { CYCLE } \\
\end{array}$} & \multicolumn{5}{|c|}{ FULL FLOW NOZZLE } \\
\hline & & 611 & 612 & 613 & 614 & 615 \\
\hline DESIGN & PROVEN & $\begin{array}{c}\text { MOST } \\
\text { COMPLEX }\end{array}$ & $\begin{array}{l}\text { LEAST } \\
\text { COMPLEX }\end{array}$ & COMPLEX & COMPLEX & COMPLEX \\
\hline FABRICATION & PROVEN & $\begin{array}{c}\text { MOST } \\
\text { COMPLEX }\end{array}$ & $\begin{array}{l}\text { LEAST } \\
\text { COMPLEX } \\
\end{array}$ & COMPLEX & COMPLEX & COMPLEX \\
\hline PERFORMAMCE & REF. & \multicolumn{5}{|c|}{ HIGHER FOR SAME AREA RATIO } \\
\hline RELIABILITY & REF. & LOWER & SAME & LOUEST & LOWER & LOWER \\
\hline WEIGHT & REF. & \multicolumn{5}{|c|}{ LOWER FOR SAME PERFORMANCE } \\
\hline LENGTH & REF. & \multicolumn{4}{|c|}{ SHORTER FOR SAME PERFORMANCE } & $\cdot$ \\
\hline COST, HOZZLE HDW & REF. & HIGHER & Un:men & HIGHEST & HIOHER & HICHER \\
\hline MAINTAILABILITY & PROVEN & BETTER & DESTF & MaRsT & EqumL? & BETTER \\
\hline $\begin{array}{l}\text { CHEMICAL SIMULATION } \\
\text { TESTING REQUIRED }\end{array}$ & YES & YES & No & NO & No & YES \\
\hline
\end{tabular}




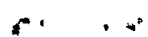


- PLUG NOZZLE CONGEPT

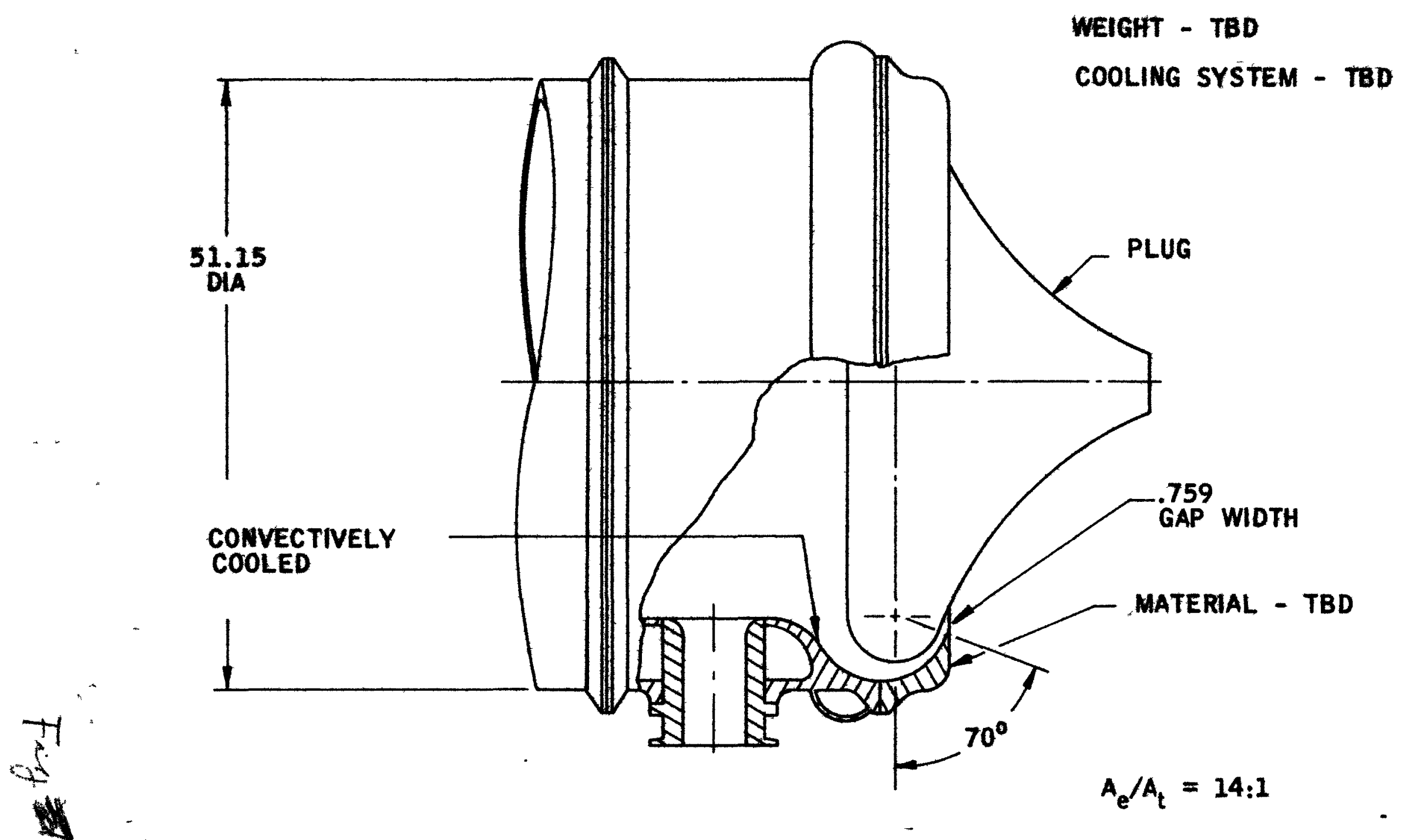




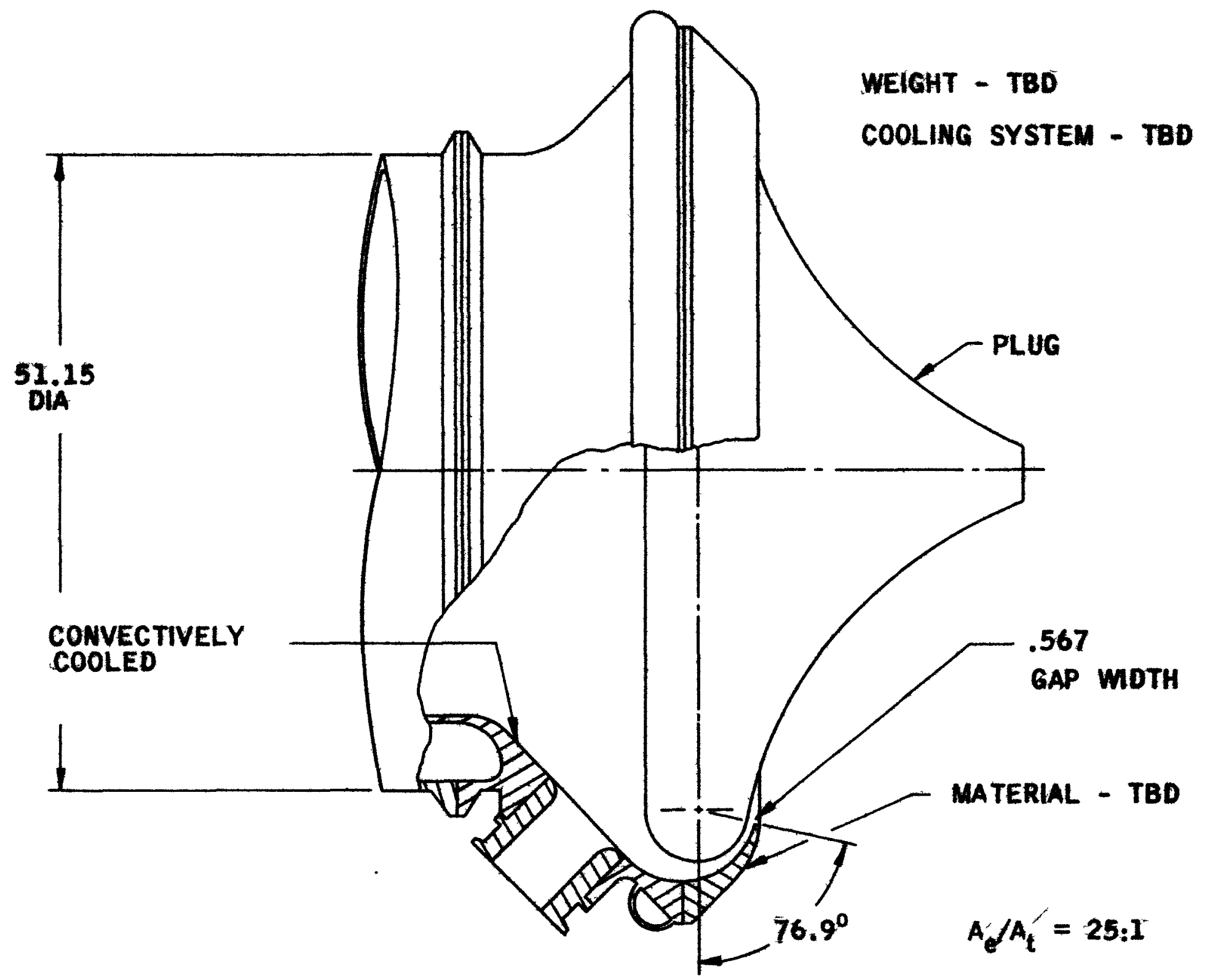




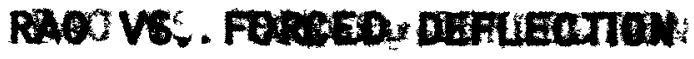
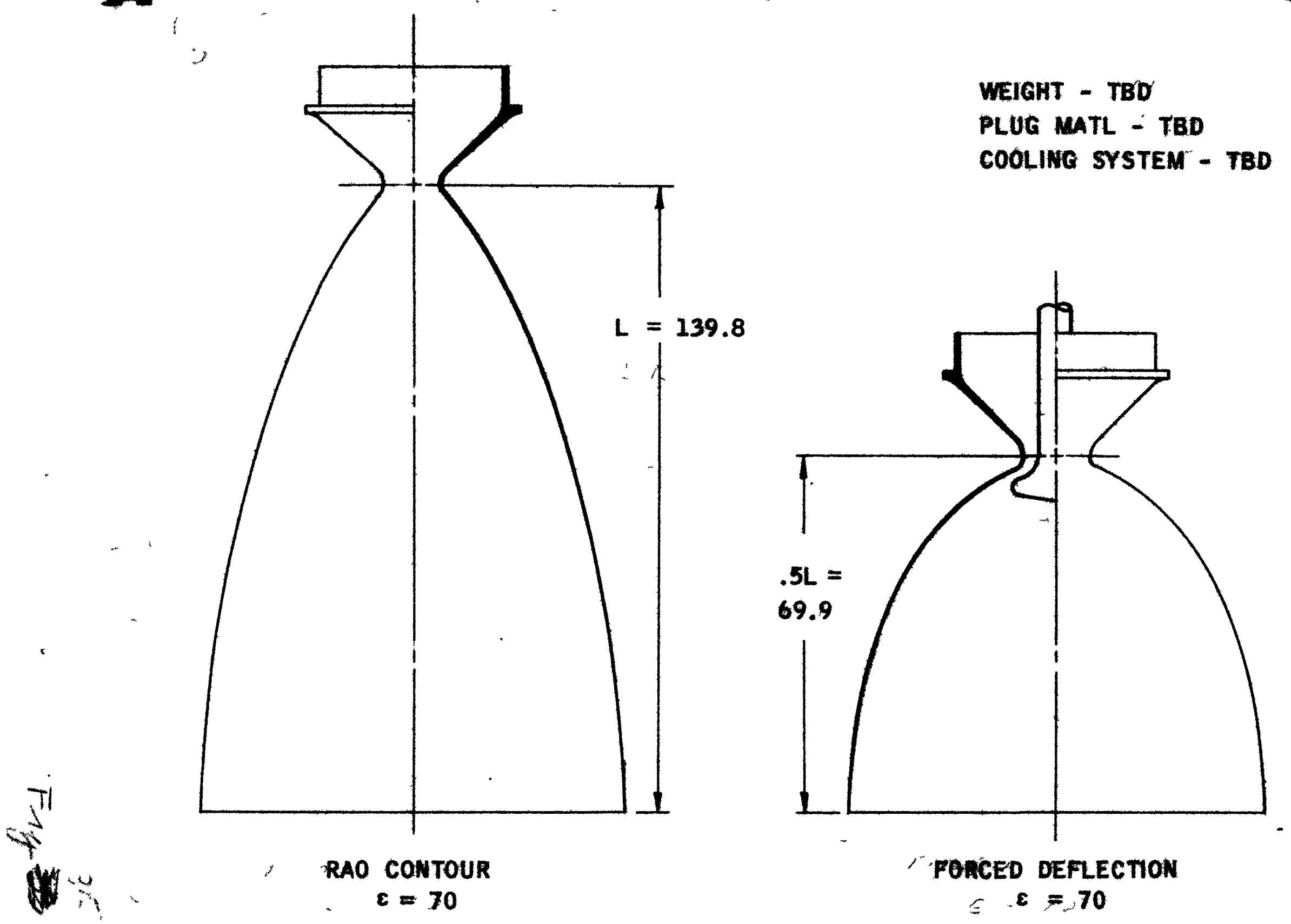


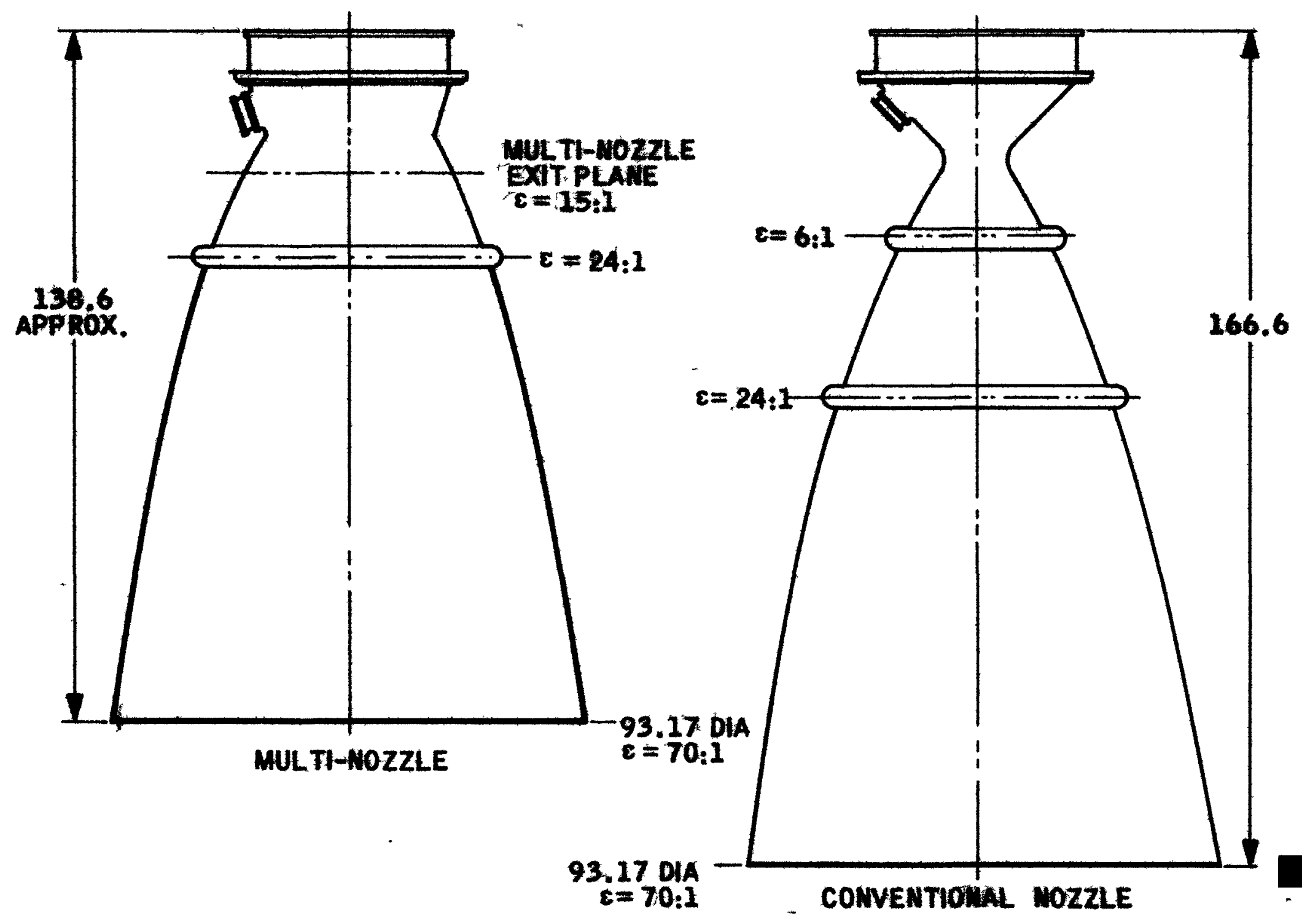


EQUIVALENT GAS SIDE DIAMETER-CHAMBER PRESSURE RELATIONSHIP WITH CONSTANT T wg MAX \& MACH NO.

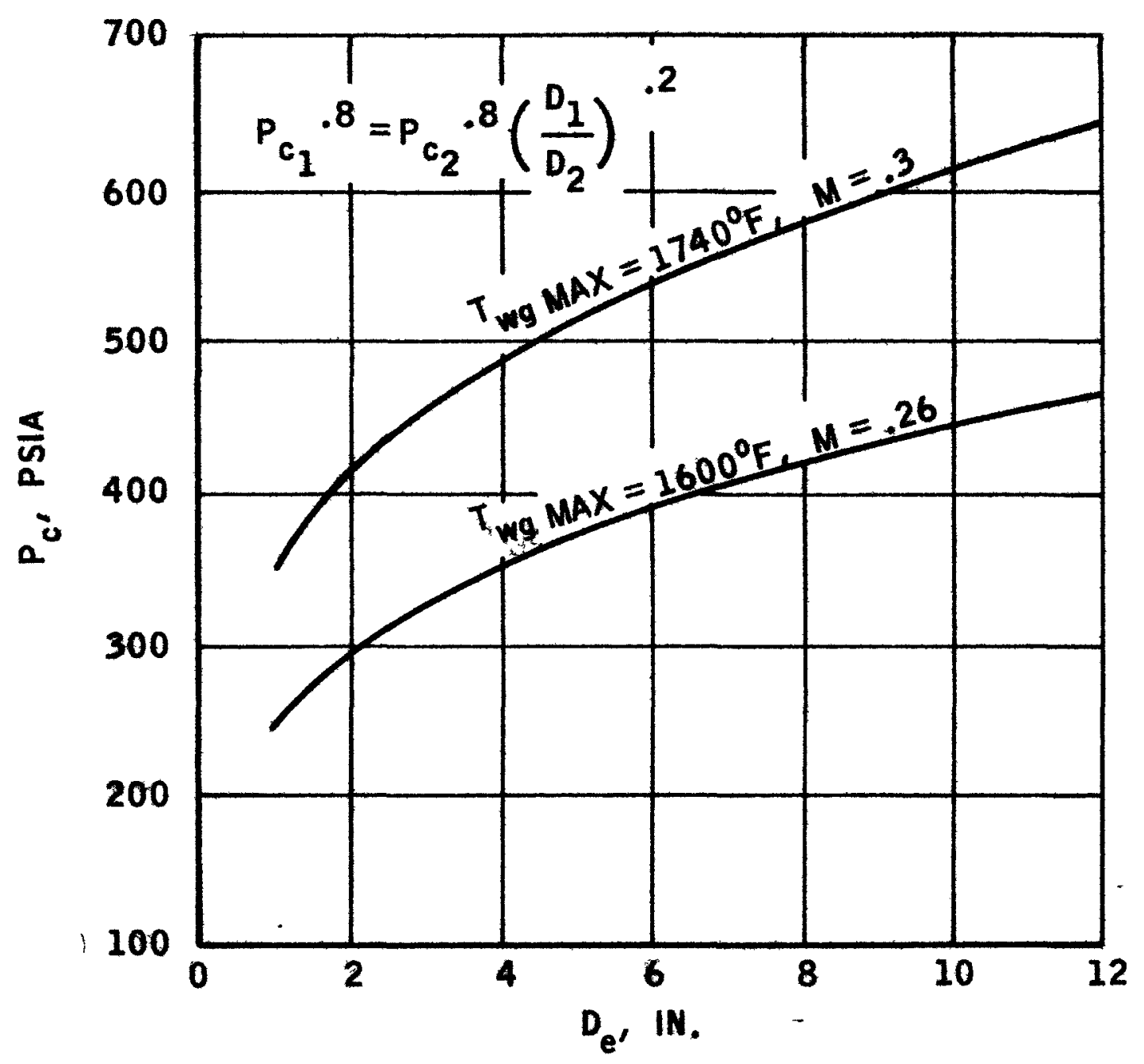




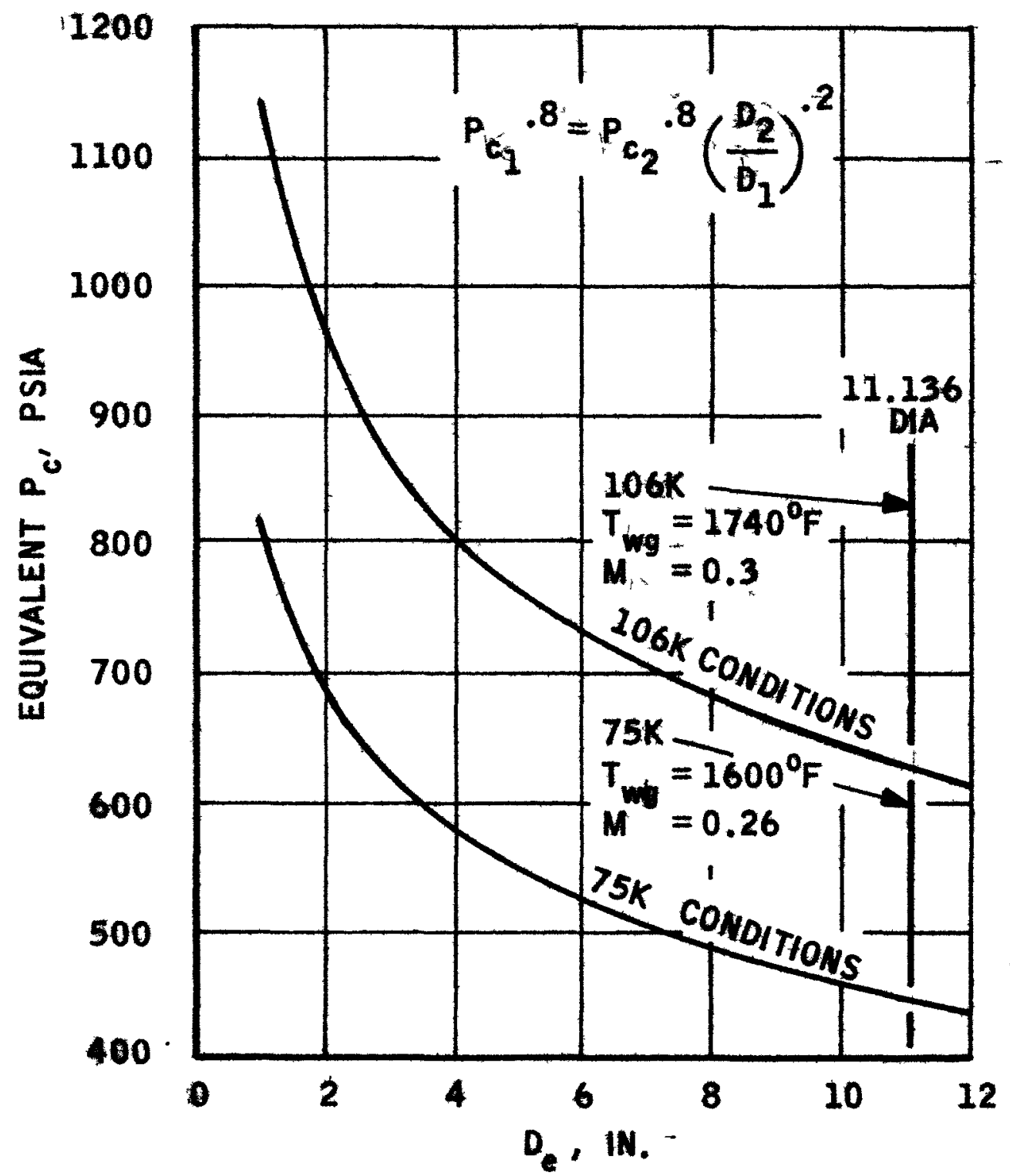


A6g.9.10

FUICITIONAT, AND

TECTINICAL DDSIGN

FEQUIREALIMTS

Earour

WEIGHT, LBB $(\varepsilon=14: 1)$

STATE OF TECHNOLOGY

NEW MAT' $Y$ REQUIRED

FAB. MAT'I, AVATLABLE

DESICN COMPEEXITY

DEVRLOPMEIT TTME

PERFORMANCT

MAXIMUM $E$ ATTAINABLE

WITHIN PV FIVVELOFE

COOLING PROBLEM

SUITABLE FOR TEST AT

ETS- 1 W/O MODIFICATION

TO FACIIITY

SOURCE OF SECONDARY

RADIATTON SCATTER

*ADDITIONAL LOSS OF I\% WHEN COMPARED WITH 100: 1 RAO. **TO RE DETERMINED.
ALTERNATE NOZZLE CONCEPTS

COMPARISON MATRIX OF DESIGN CONCEPTS

\begin{tabular}{|c|c|c|c|c|}
\hline SPIKE & MULTIPLE & $\begin{array}{c}\text { FORCED } \\
\text { DEFLECTION }\end{array}$ & CONIC & RAO \\
\hline $30 \%$ & $80 \%$ & $50 \%$ & $110 \%$ & REF. \\
\hline-300 & +500 & -200 & -100 & REF. \\
\hline LIMITED & NII & NIL & DEVELOPED & DEVEIOPED \\
\hline YES & YES & YES & no & no \\
\hline no & no & No & YES & YES \\
\hline COMPLEXX & COMPLEXX & COMPTIEX & STATE-OF-ART & STATT-OF-ART \\
\hline $\begin{array}{l}\text { SIGNI FICANTLY } \\
\text { LONGER }\end{array}$ & $\begin{array}{l}\text { SIGNI FICANTLY } \\
\text { LONGER }\end{array}$ & $\begin{array}{l}\text { SIGNI FICANTIY } \\
\text { IONGER }\end{array}$ & SAME AS REF. & REF. \\
\hline $\begin{array}{c}-5 \% \text { at } \varepsilon= \\
14: 1\end{array}$ & $\begin{array}{c}-2.0 \% \text { at } \varepsilon= \\
70: 1\end{array}$ & $\begin{array}{c}-1.0 \% \text { at } \varepsilon= \\
70: 1\end{array}$ & $\begin{array}{c}*-1.0 \% \text { at } \varepsilon= \\
70: 1\end{array}$ & $\begin{array}{l}\text { REF. } \varepsilon \\
=70: 1\end{array}$ \\
\hline 14 & NO INFLUENCE & NO INFLUENCE & NO INFLUENCE & NO INFLUENCE \\
\hline DIFFICULT & DIFFICULT & DIFFICULT & STATE-@F-ART & STATE-OF-ART \\
\hline TBD** & $\begin{array}{c}\text { YES } \\
\text { (TRUNCATED) }\end{array}$ & $\begin{array}{c}\text { YES } \\
\text { (TRUNCATED) }\end{array}$ & $\begin{array}{c}\text { IES } \\
\text { (TRUNCATED) }\end{array}$ & $\begin{array}{c}\text { YFS } \\
\text { (TRUNCATED) }\end{array}$ \\
\hline TBD & SLIGHTLY LESS & LESS & MAXIMUM & REF. \\
\hline
\end{tabular}




\section{NERVA TRADE STUDIES}

NOZZLE ASSEMBLY

THEORETICAL I SP DEGRADATION SOURCES

$$
A_{e} / A_{t}=20: 1 \quad A_{e} / A_{t}=100: 1
$$

- NOZZLE EXIT LOSSES

$1.5 \%$

$0.5 \%$

- BOUNDARY LOSSES

$1.5 \%$

$2.5 \%$

THERMAL \& VISCOUS

- CONTOUR IMPERFECTIONS

INHERENT

$0.25 \%$

$0.25 \%$

DISTORTION FROM AS BUILT TO

$0.25 \%$

$0.25 \%$ OPERATION

\section{$3.5 \%$}

$3.5 \%$

$-\quad I_{s p}$ RMS $3 \sigma$ TOLERANCE \pm 4.12 SEC (APPROX $\pm 0.5 \%$ *

- FROZEN EQUILIBRIUM PAST $A_{e / A_{t}}=5: 1 ;-5$ SEC (APPROX $\leqslant-0.5 \%$ )

* TOLERANCE LIMITS APPLY TO ENGINE..

NOZZLE TOLERANCE IS $\pm 0.498 \% t_{\text {sp }}$ THEORETICAL MAIN NOZZLE. 


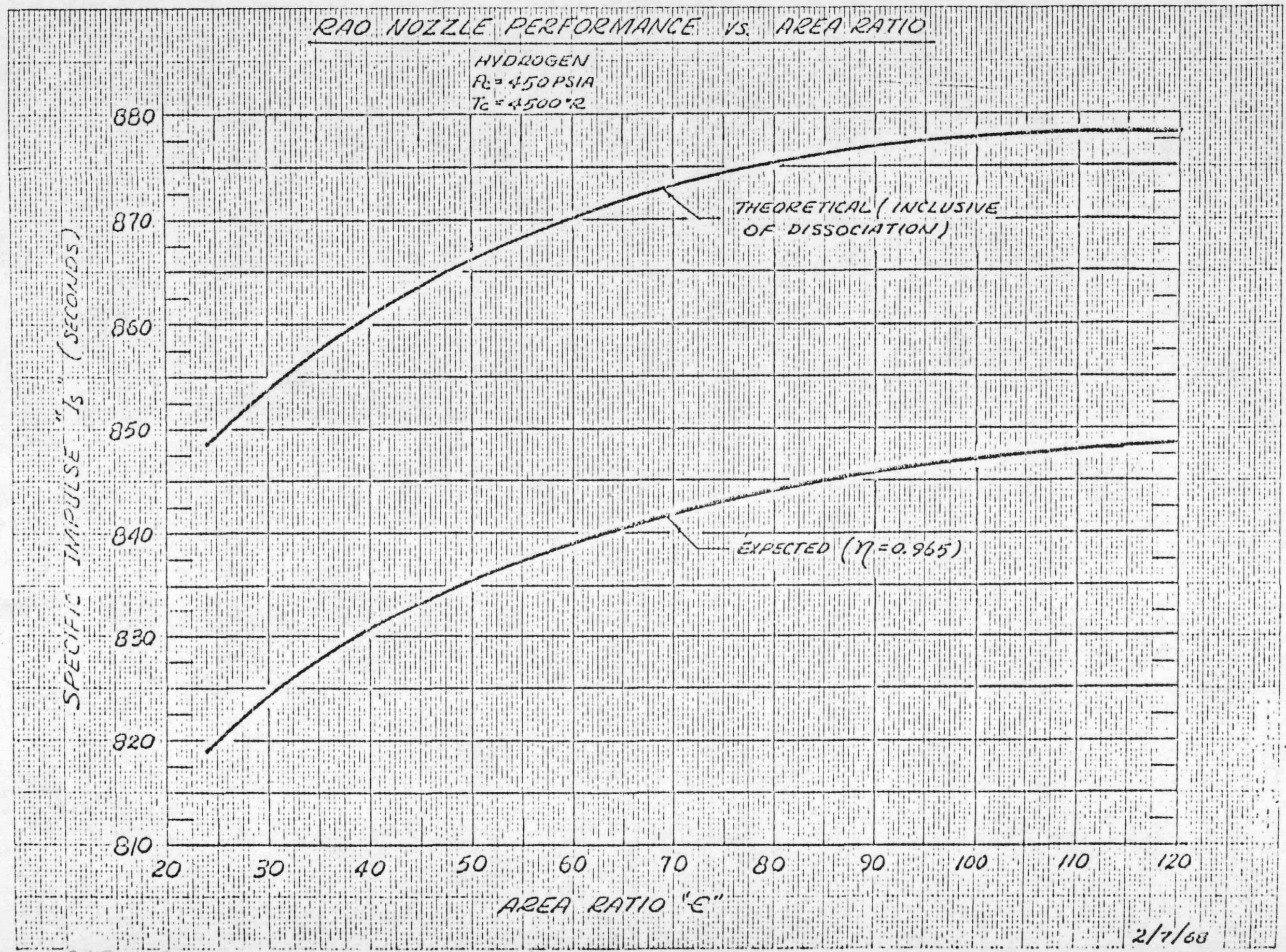




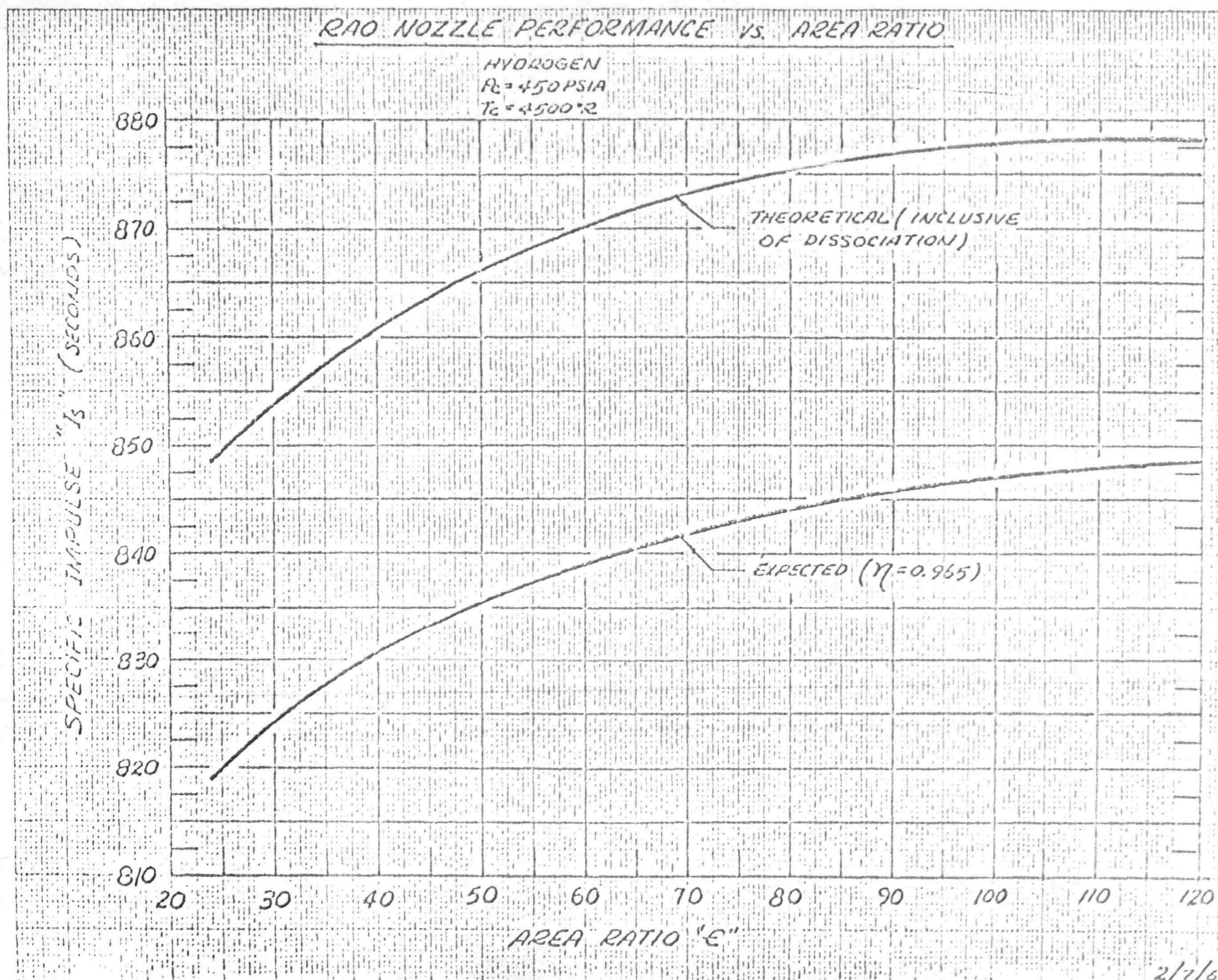




\section{unad vs of}

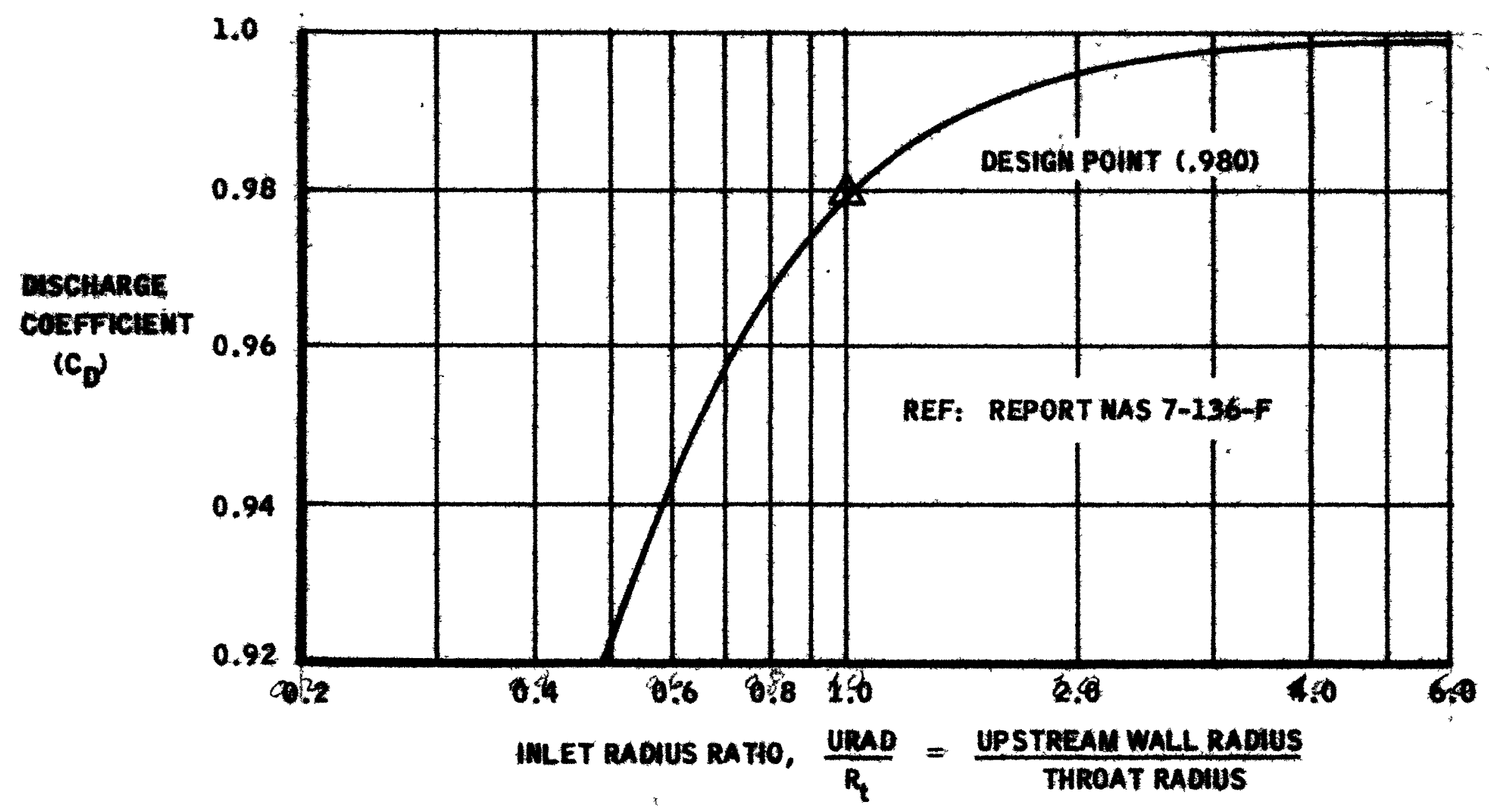




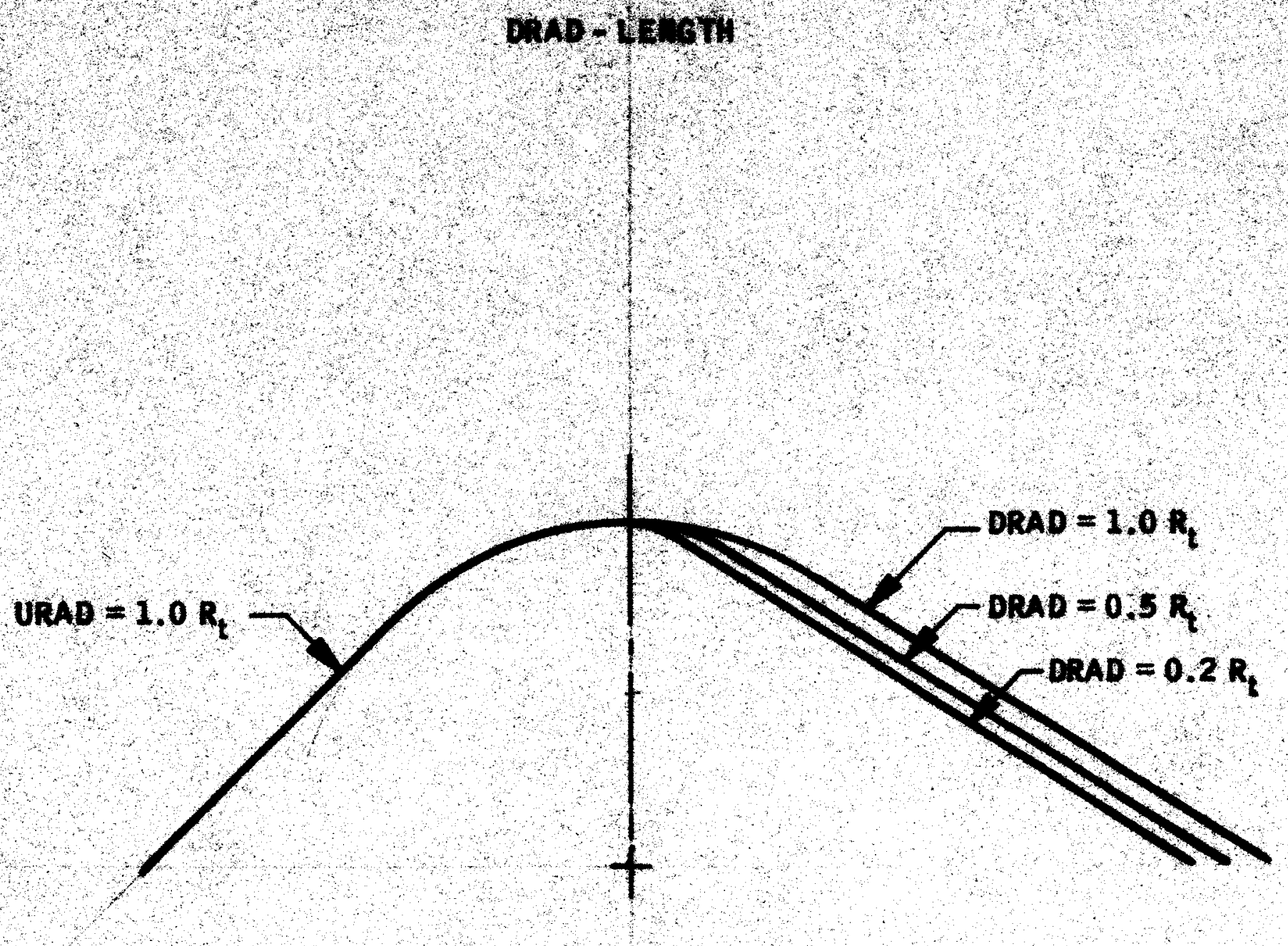

NOZZL IMSIDE CONTOUR 


\section{URAD, DRAD VS Is}






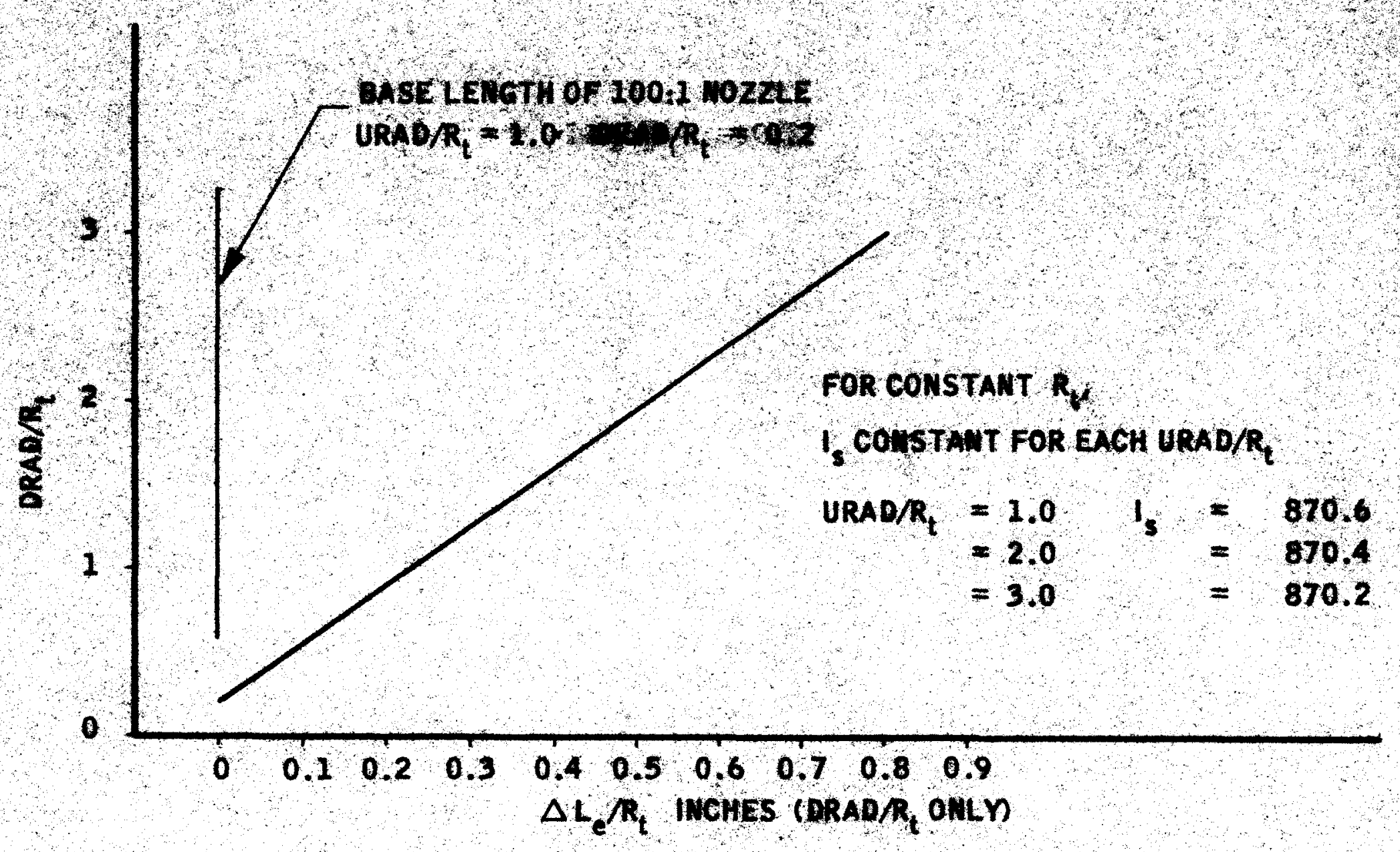




\section{WEIGHT ADVANTAGE VS $\delta$}

NET WEIGHT ADVANTAGE

( LB )

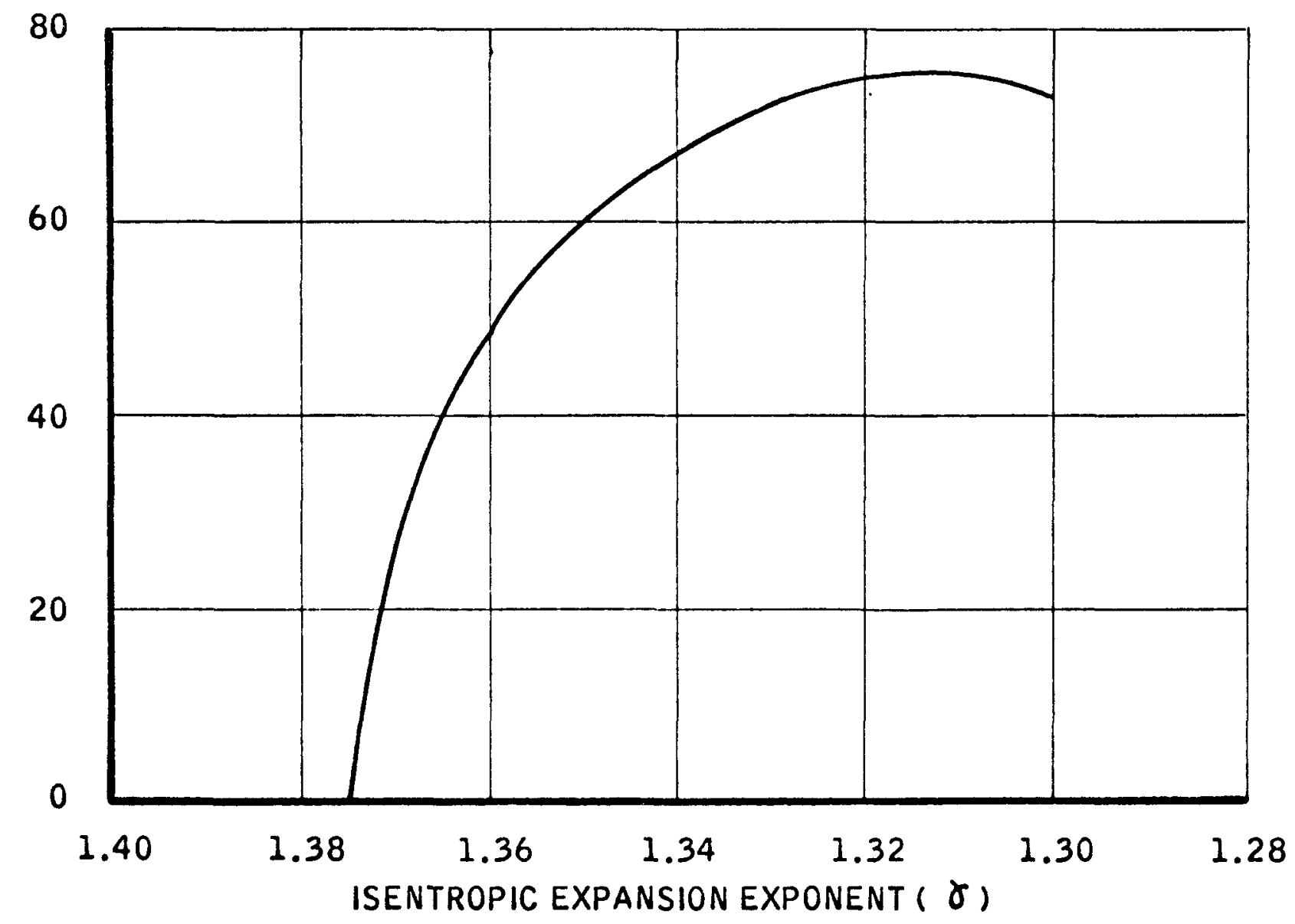


v vs 4 ,
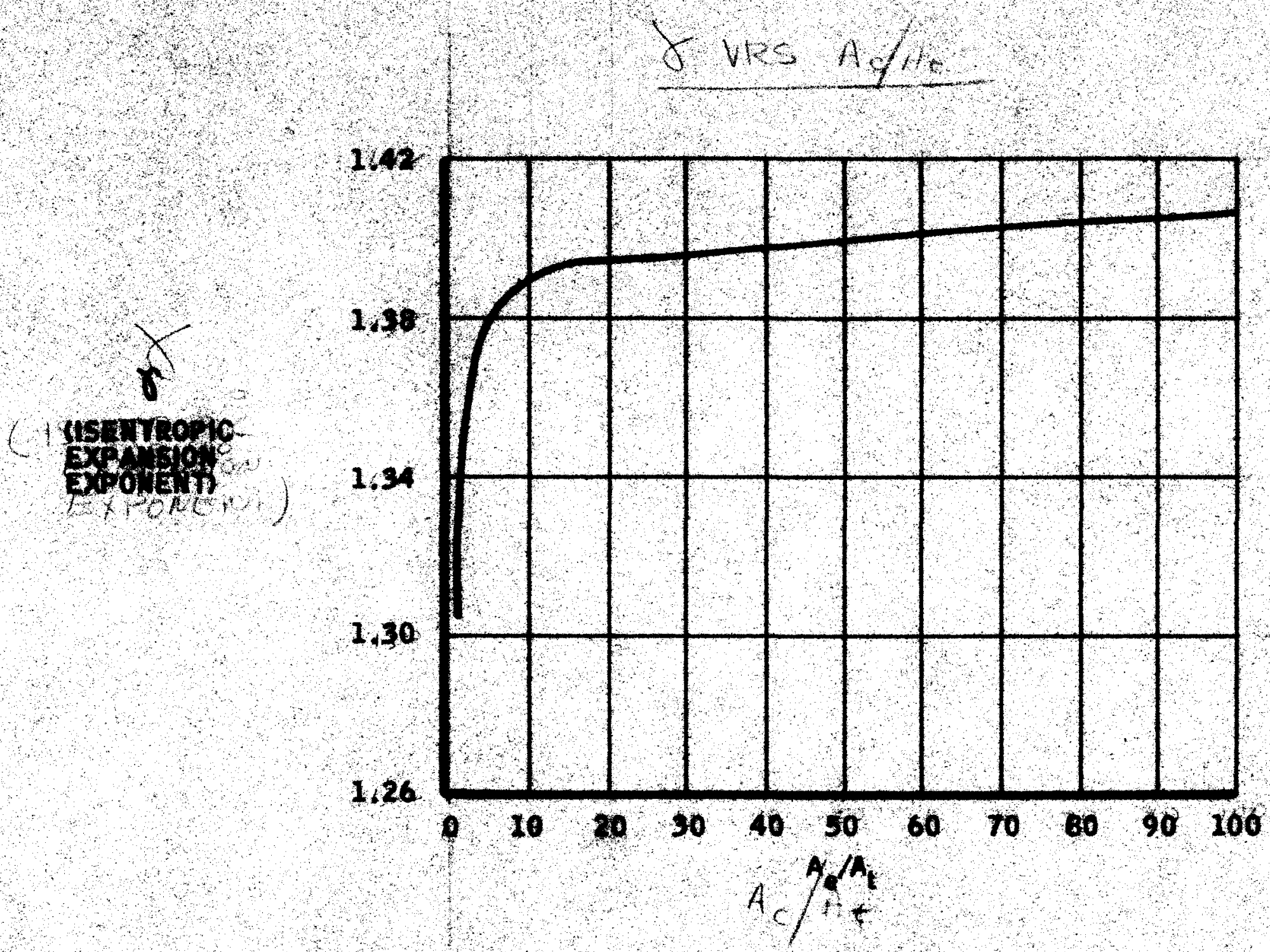

$269 \cdot 182$ 

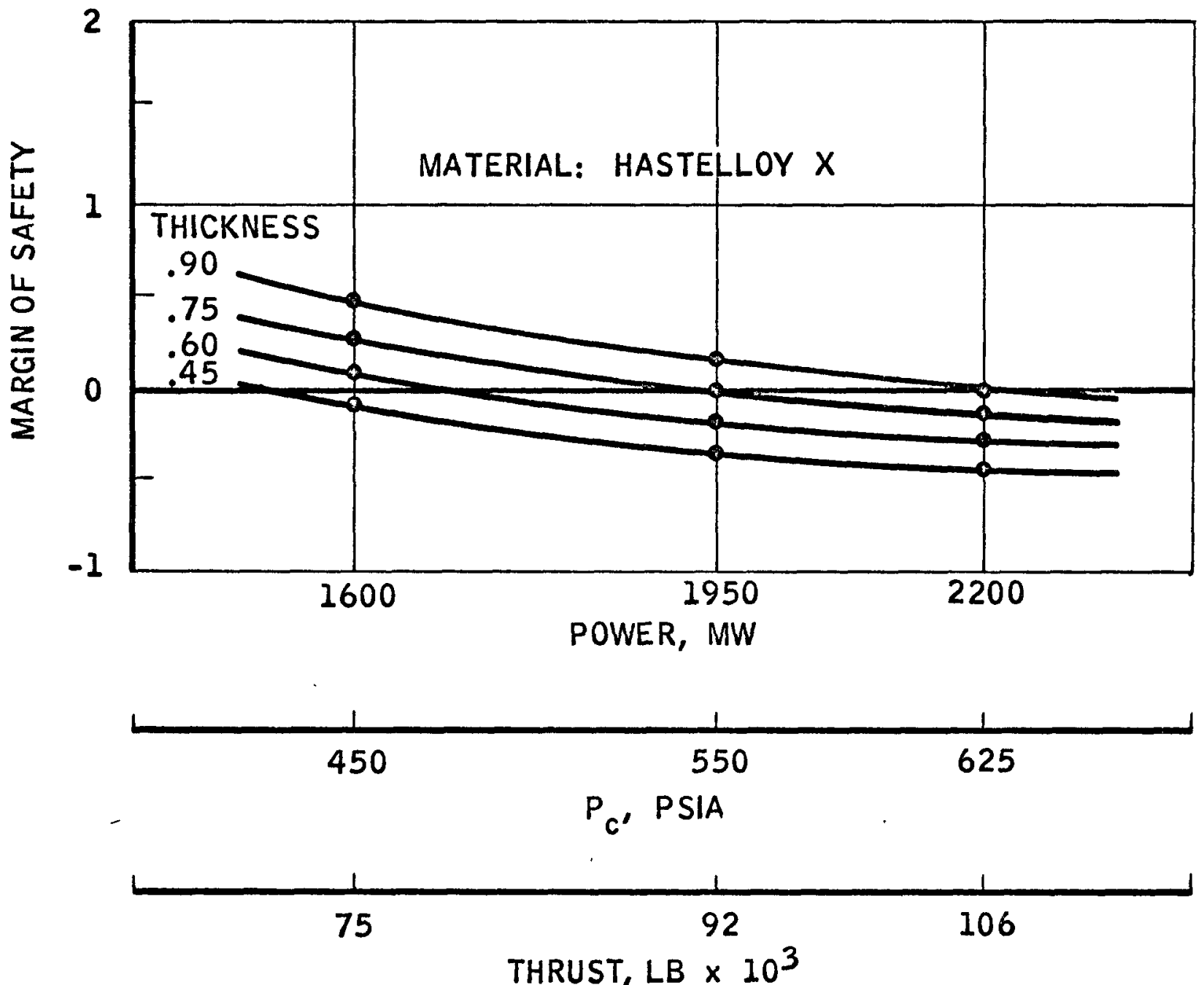
$A_{\mathrm{t}}=$ CONST

$\dot{W}$ = VARIES LINEARLY WITH F

$T_{C}=$ CONSTANT $\left(4500^{\circ} \mathrm{R}\right)$

CORE SUPPORT FLANGE DIA

CONVERGENCE ANGLE CONSTANT

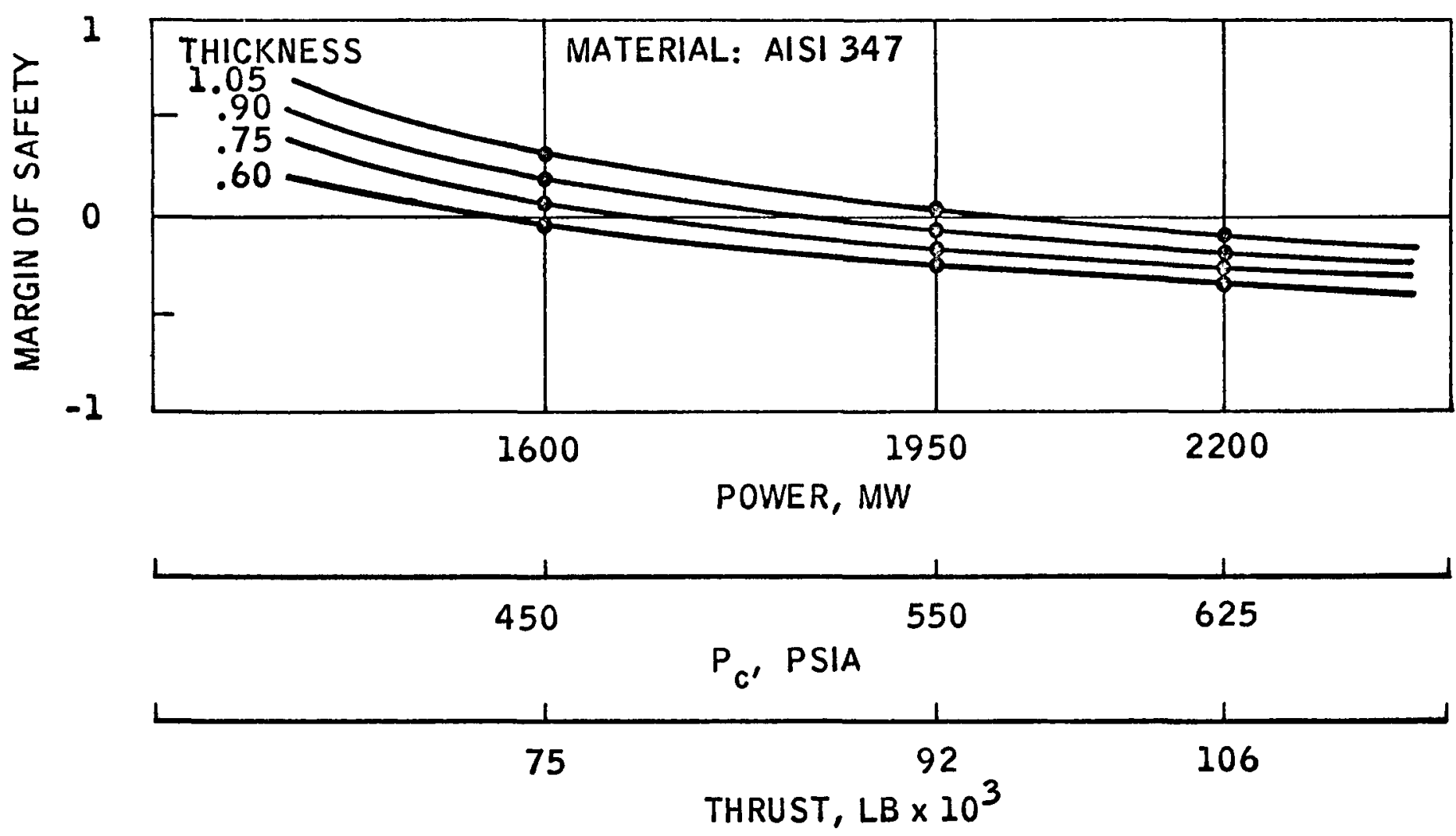


agc-pino

NOZZLE MATERIALS TRADE STUDY

COOLANT CHANNEL MARGIN OF SAFETY

(OPER)

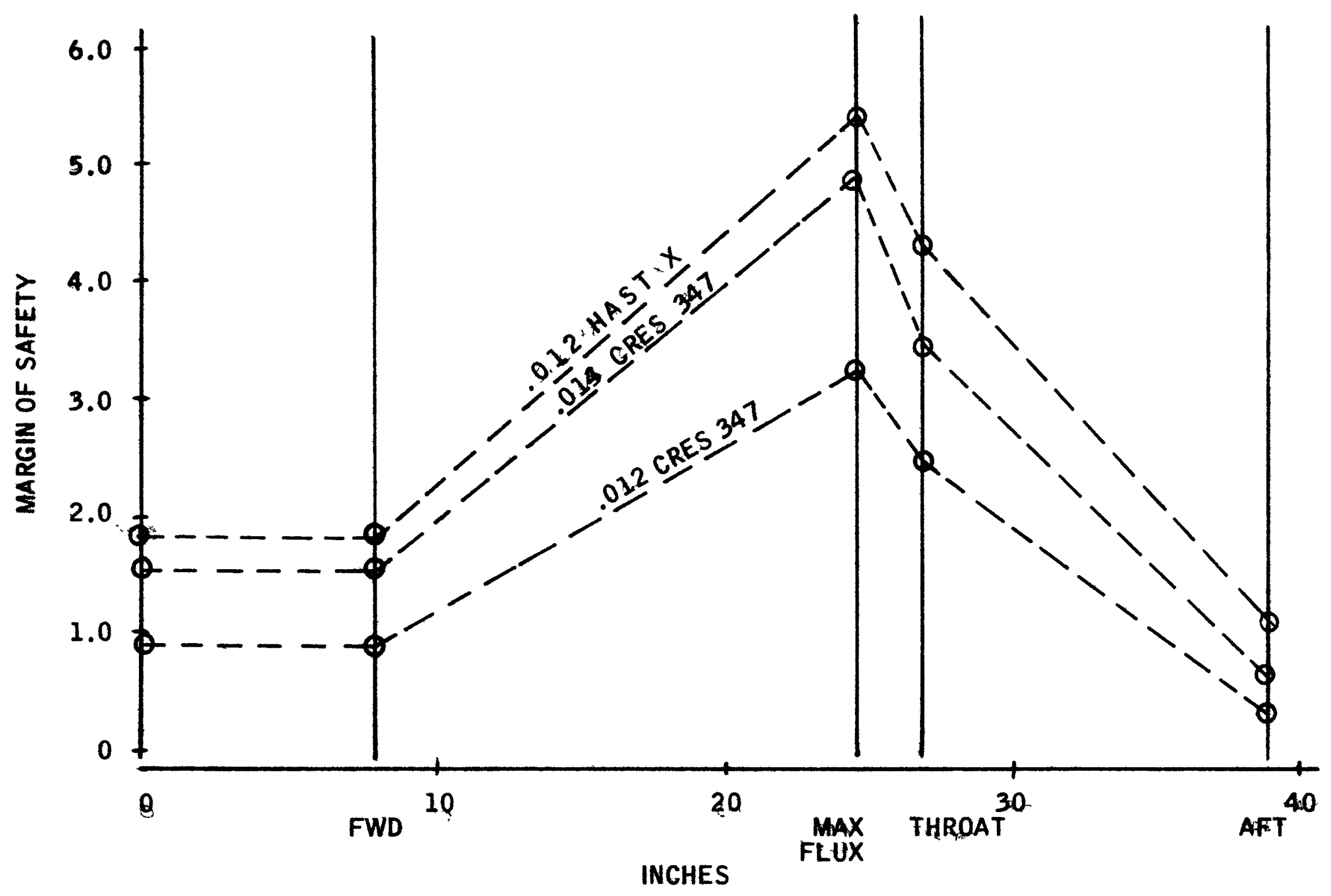

269150 
Ace.p.10

NOZZLE MATERIALS TRADE STUDY

COOLANT CHANNEL MARGIN OF SAFETY

(HYDRO)

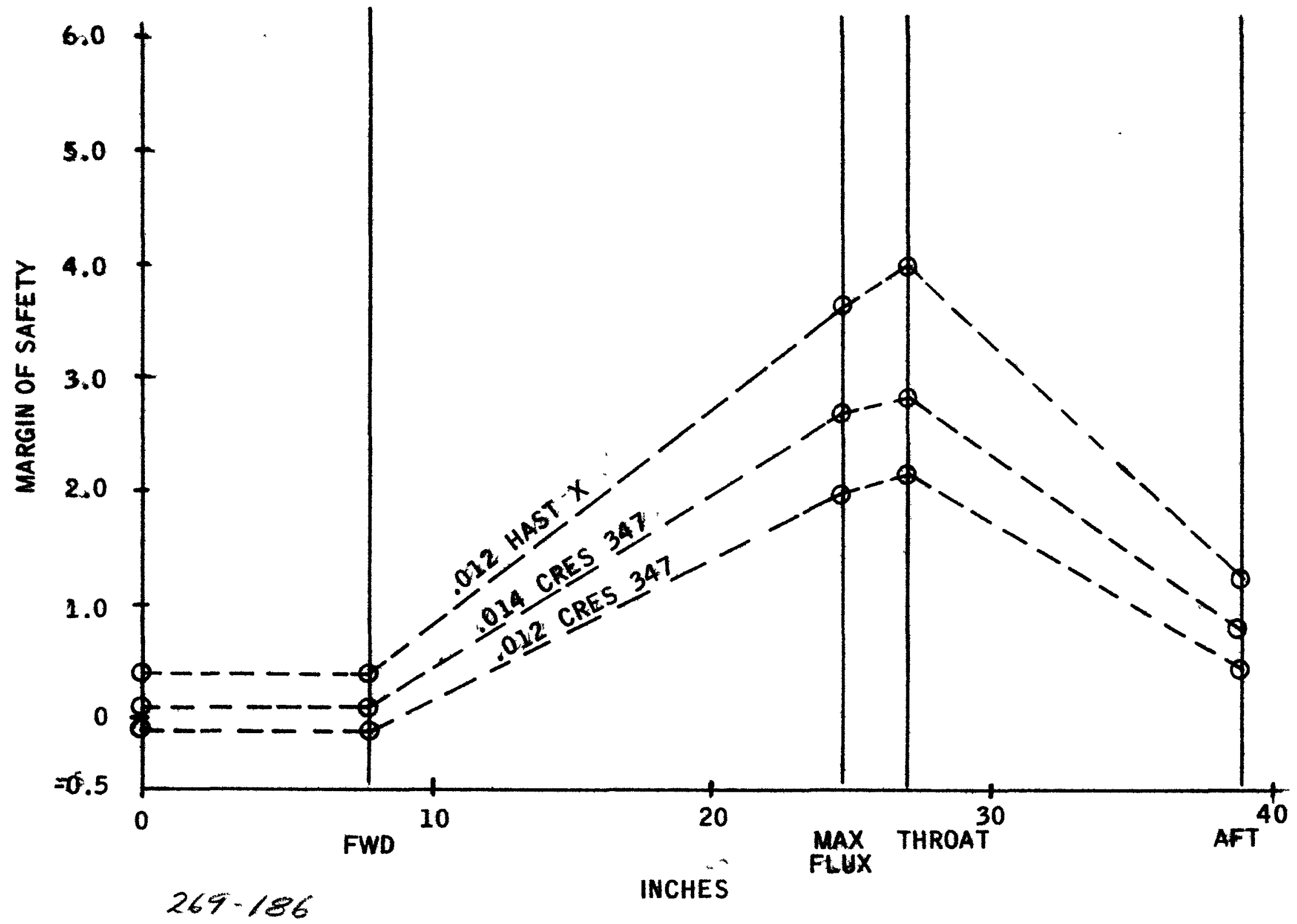




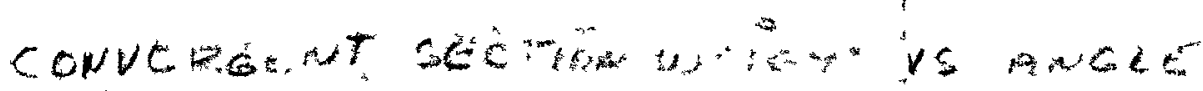

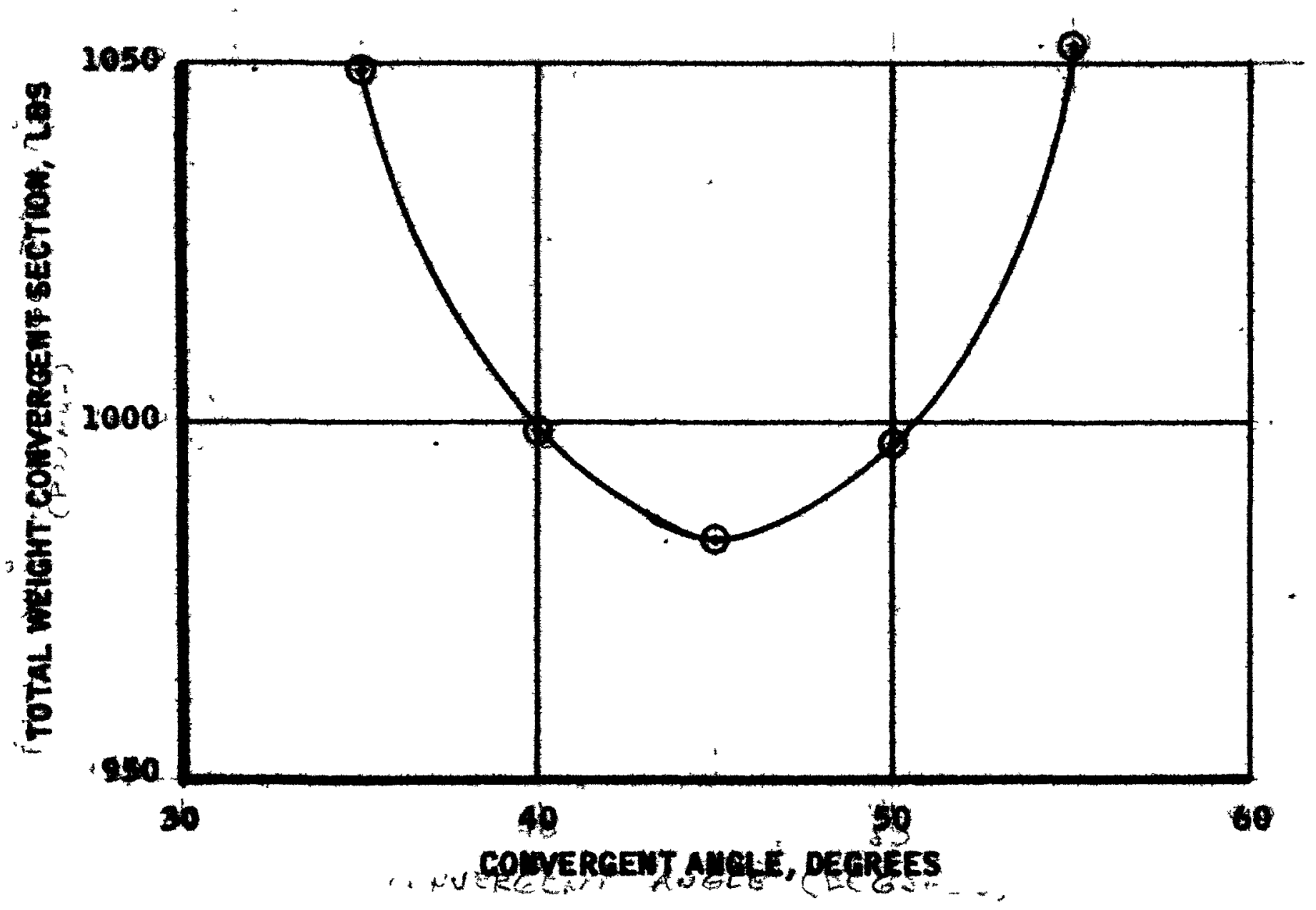




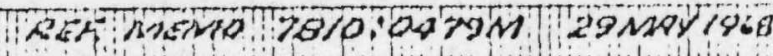

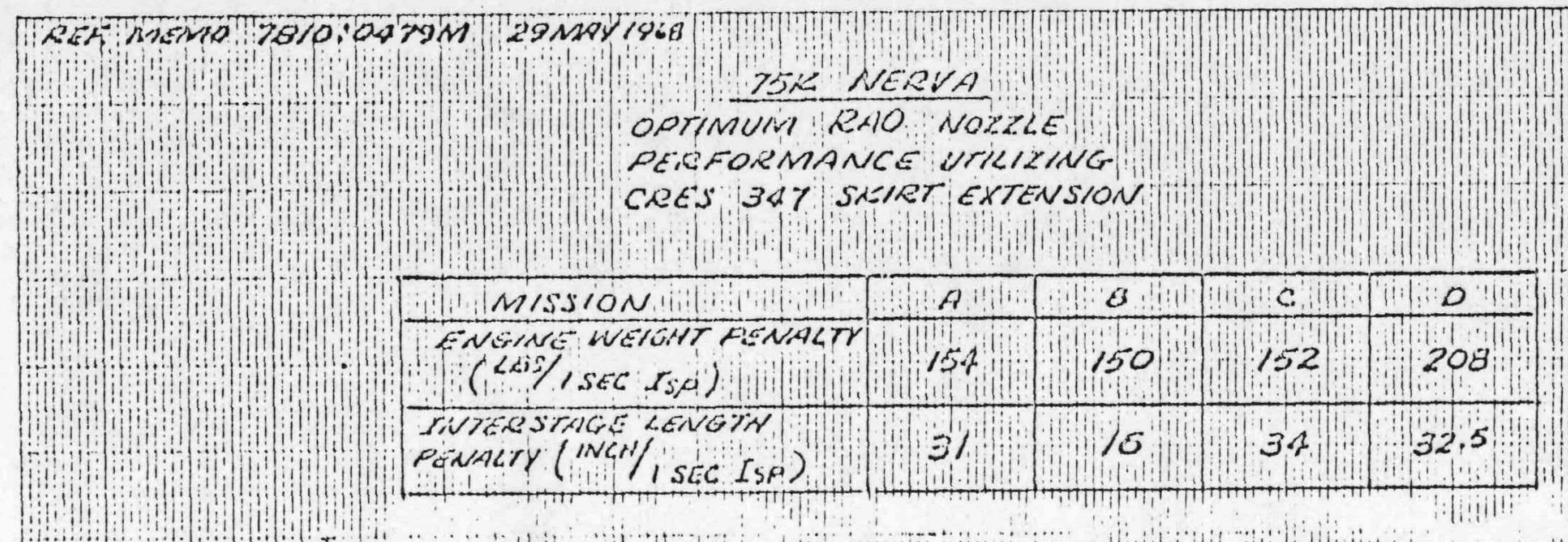

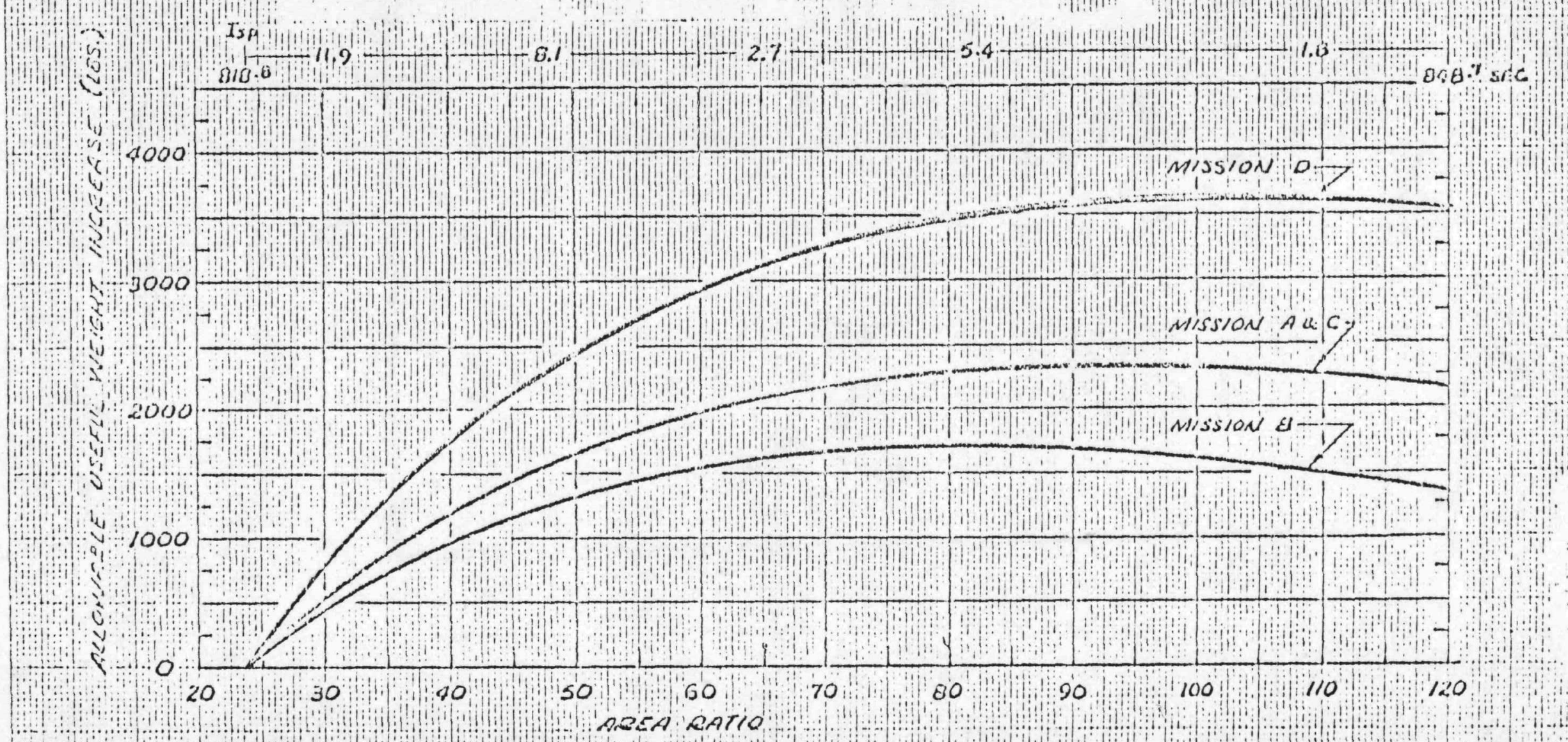
o s/át 14. 


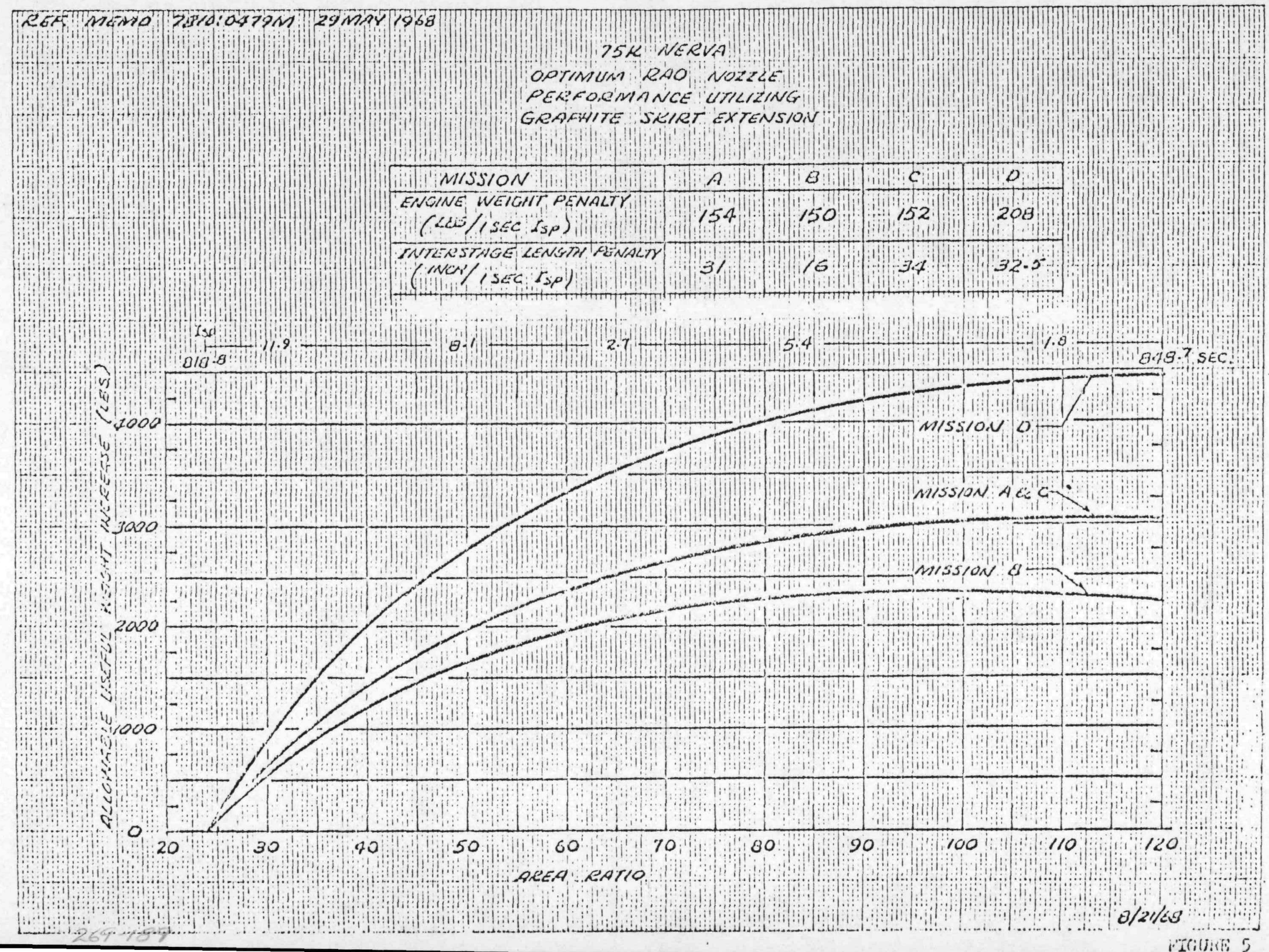




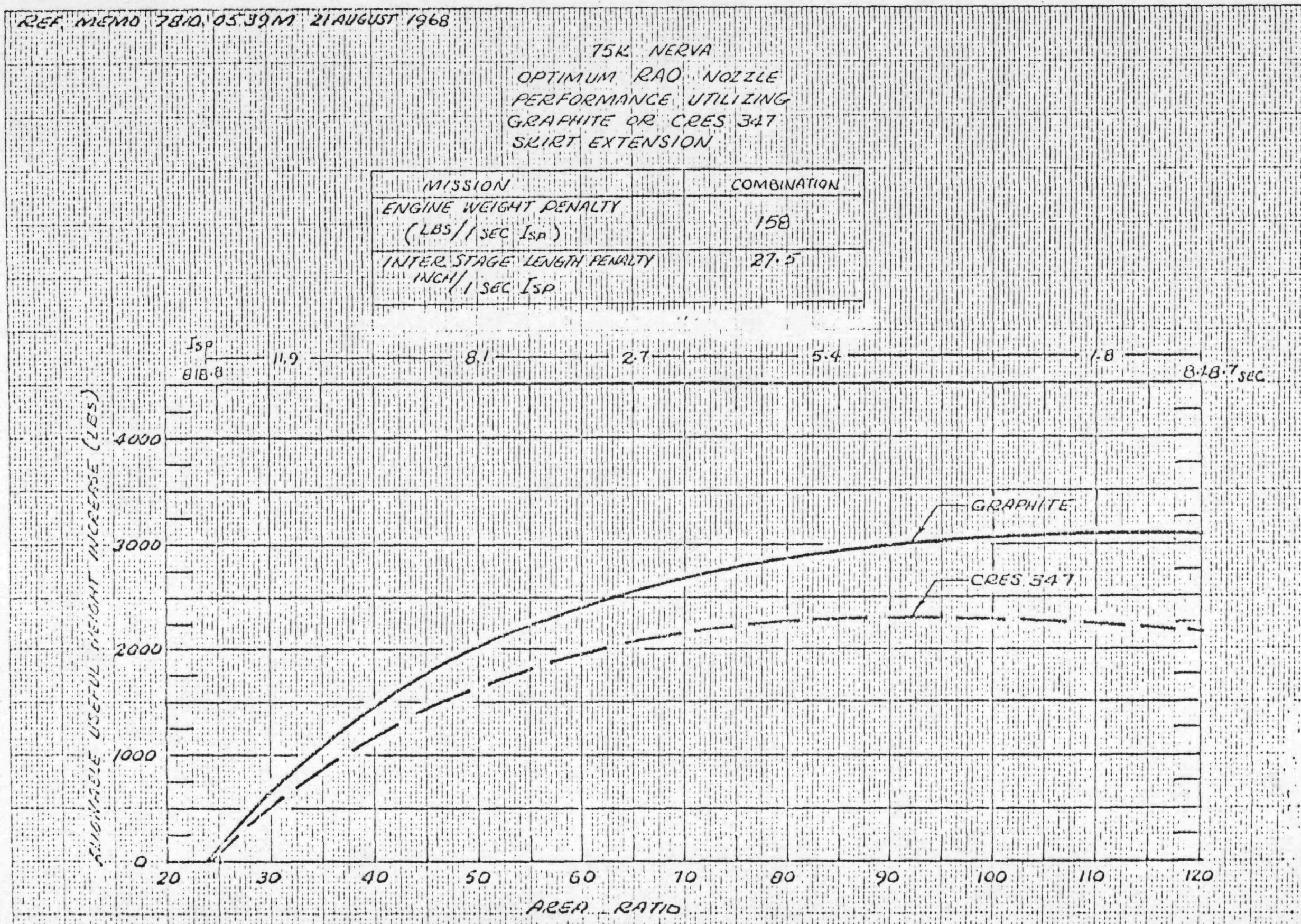


OEMAROED COOLANT (TUROUE EXLAUST) MACH MUNBER AS FUNCTION OF MOT GAS SIDE WALL TEMERATURE AND NOZZLE AREA RATHO WN THE 75K SKIRT EXTENSION GWLET (PRELIMANARV

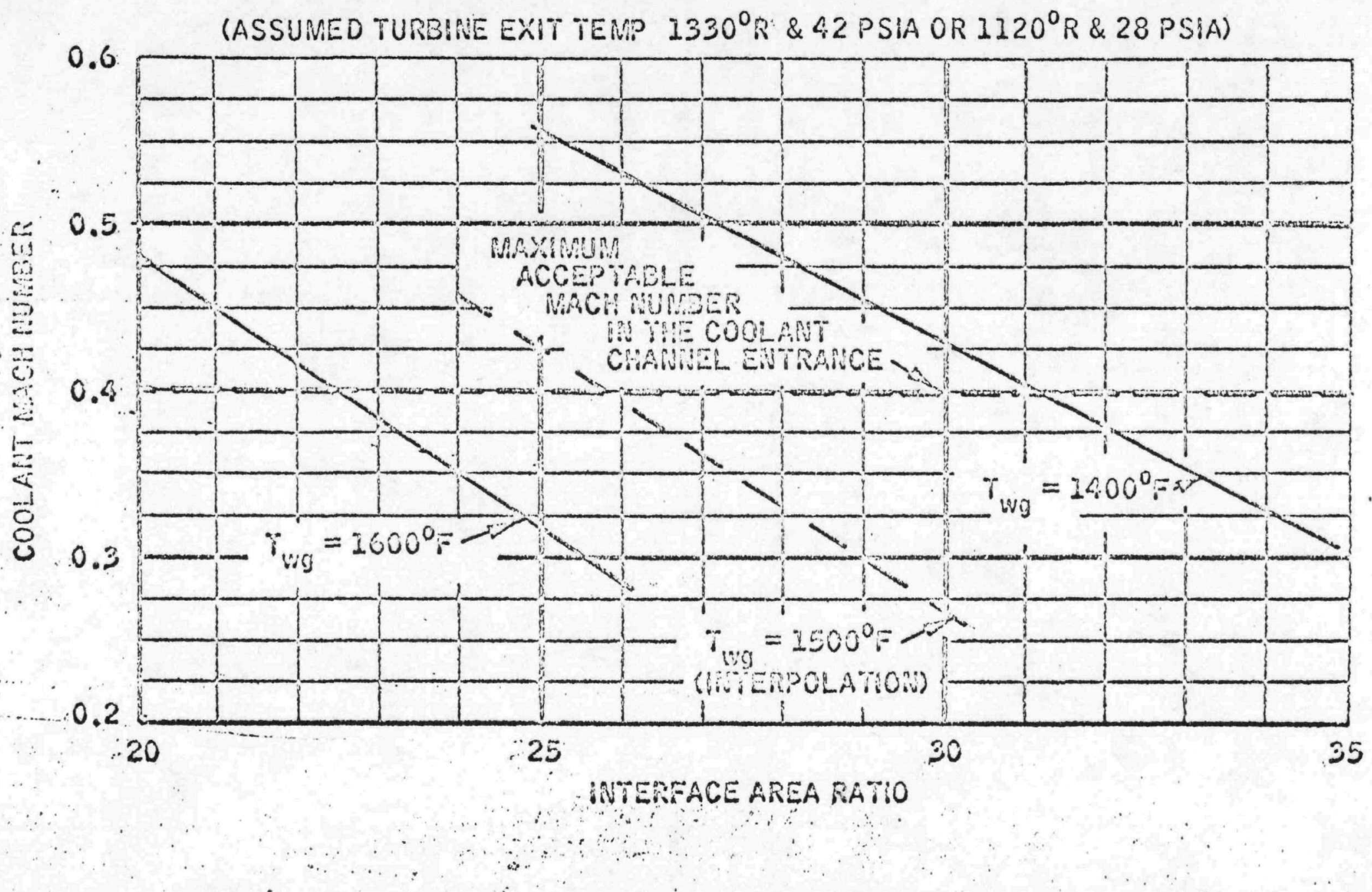




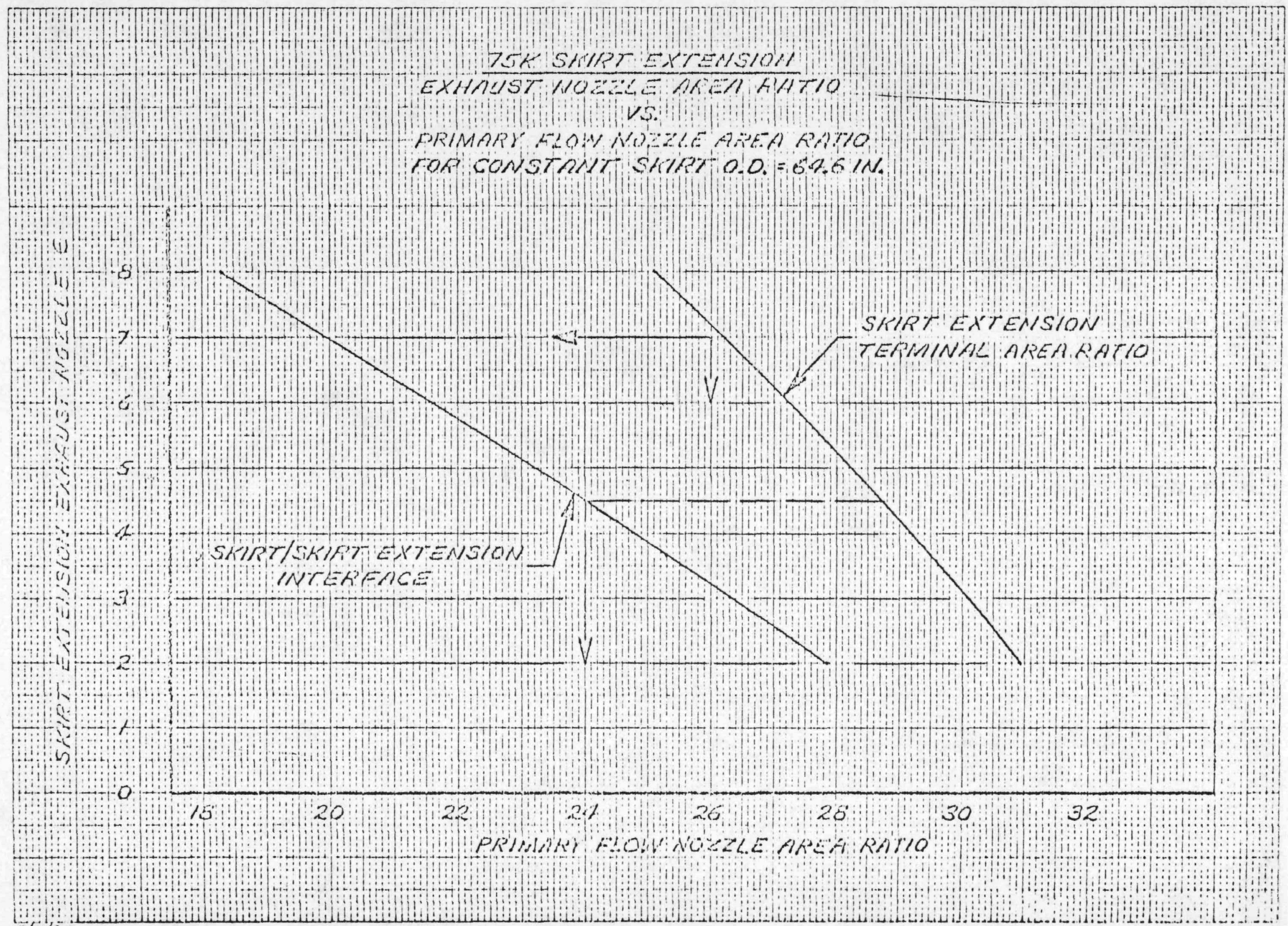




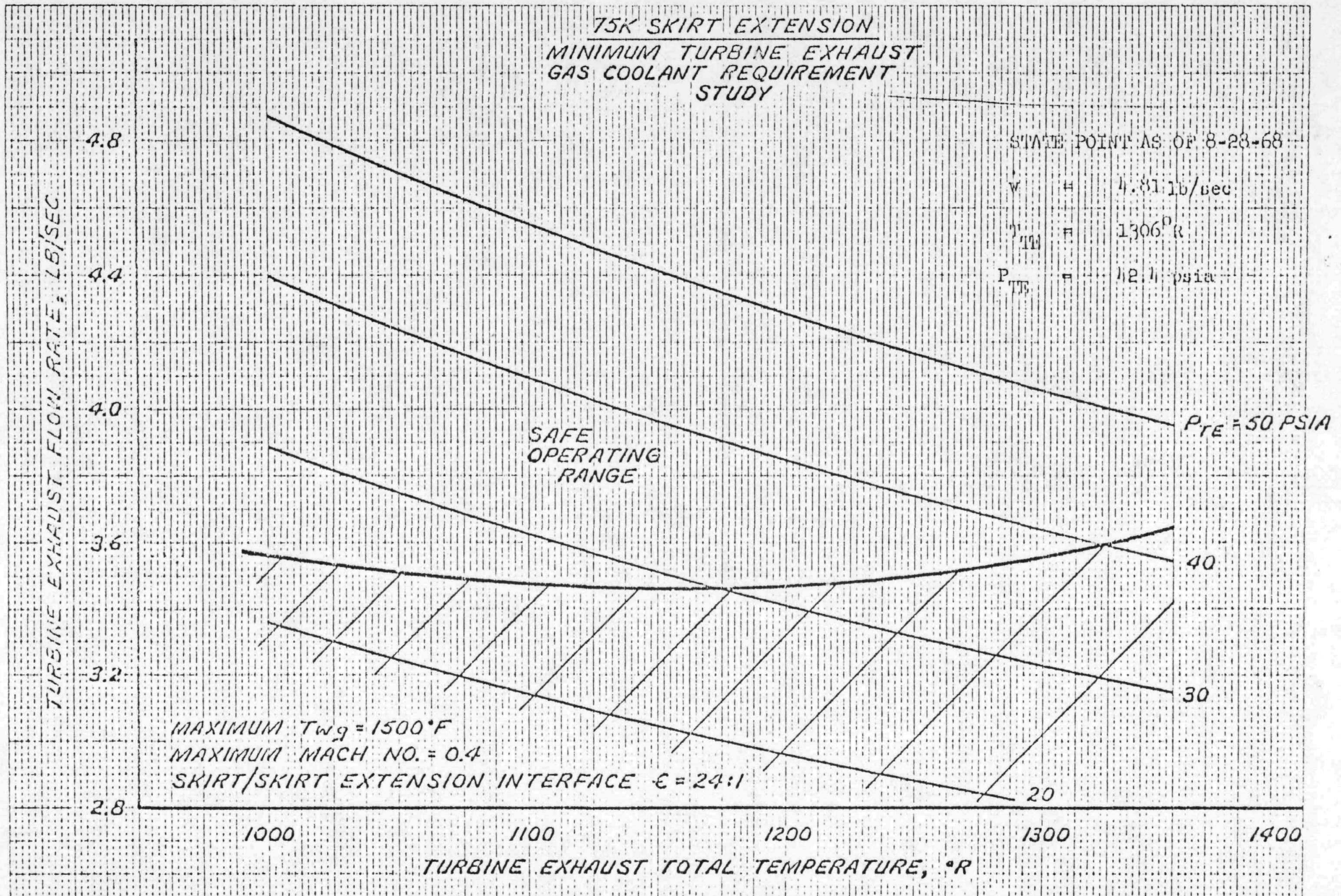




\section{NOZZLE / SKIRT \\ PARALLEL COOLANT FLOW \\ SCHEMATIC \\ (SINGLE PASS SKIRT)}

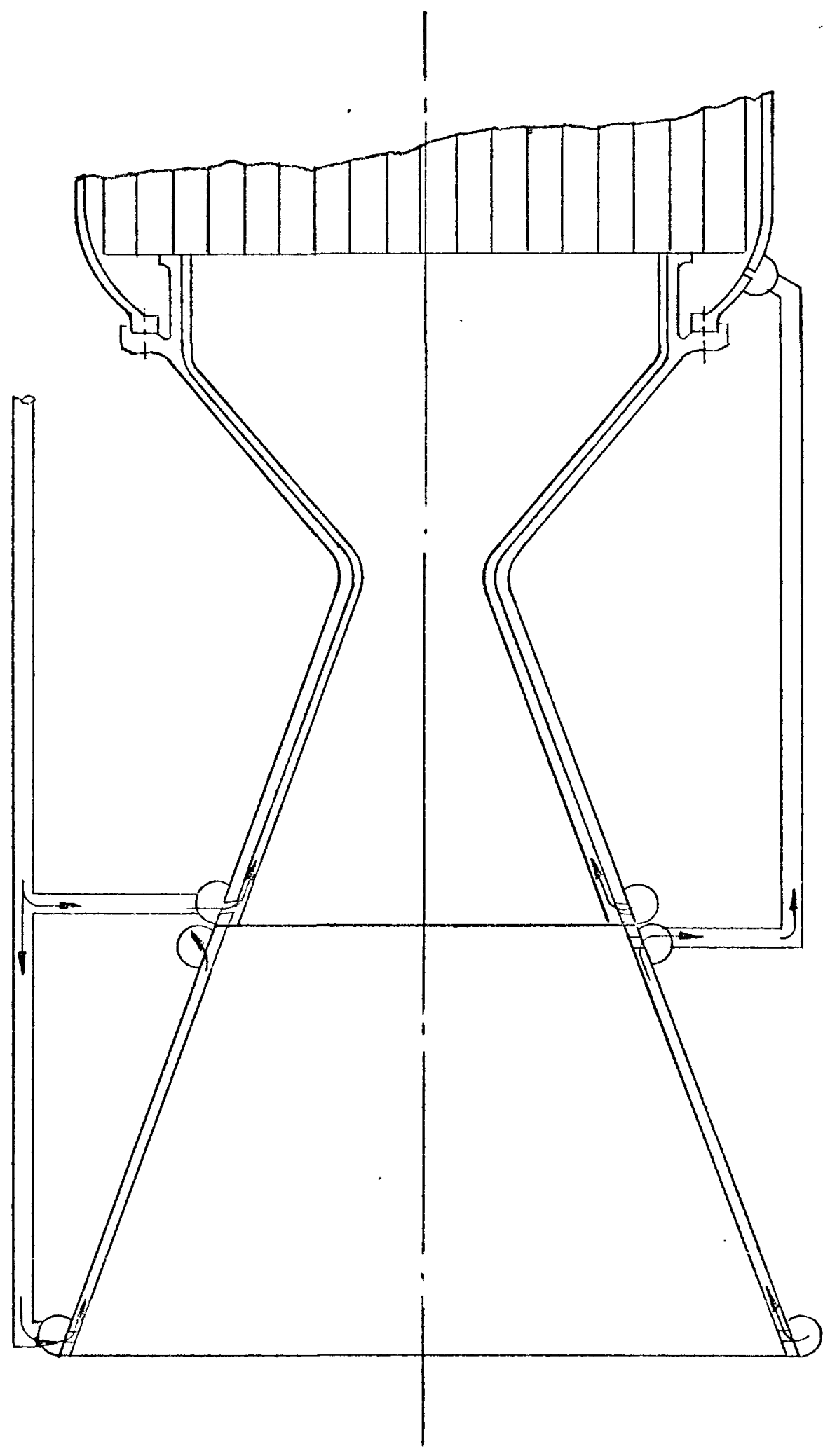

FIG. II 


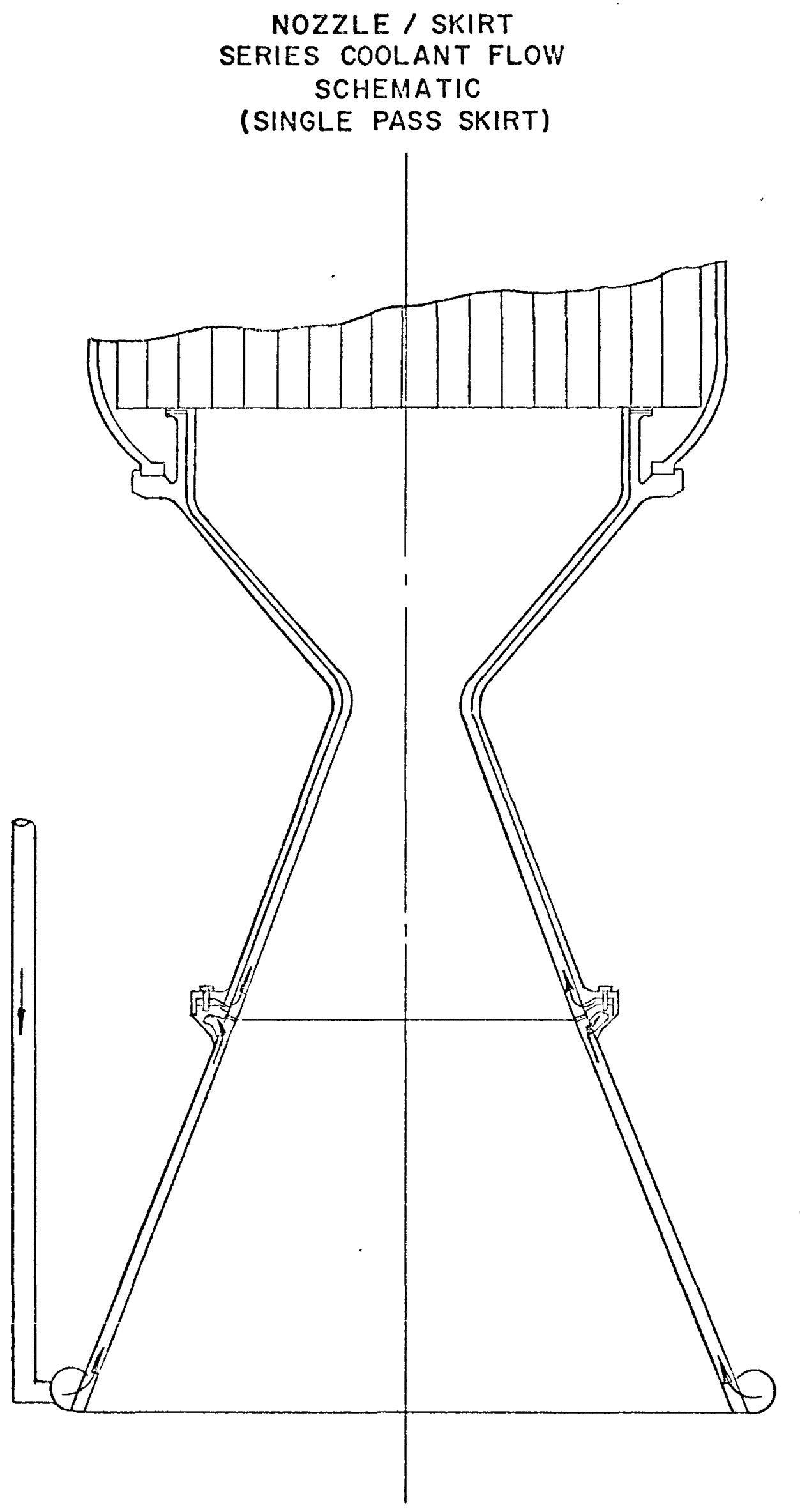

FIG. I2 


\section{NOZZLE / SKIRT \\ SERIES COOLANT FLOW \\ SCHEMATIC \\ (DOUBLE PASS SKIFT)}

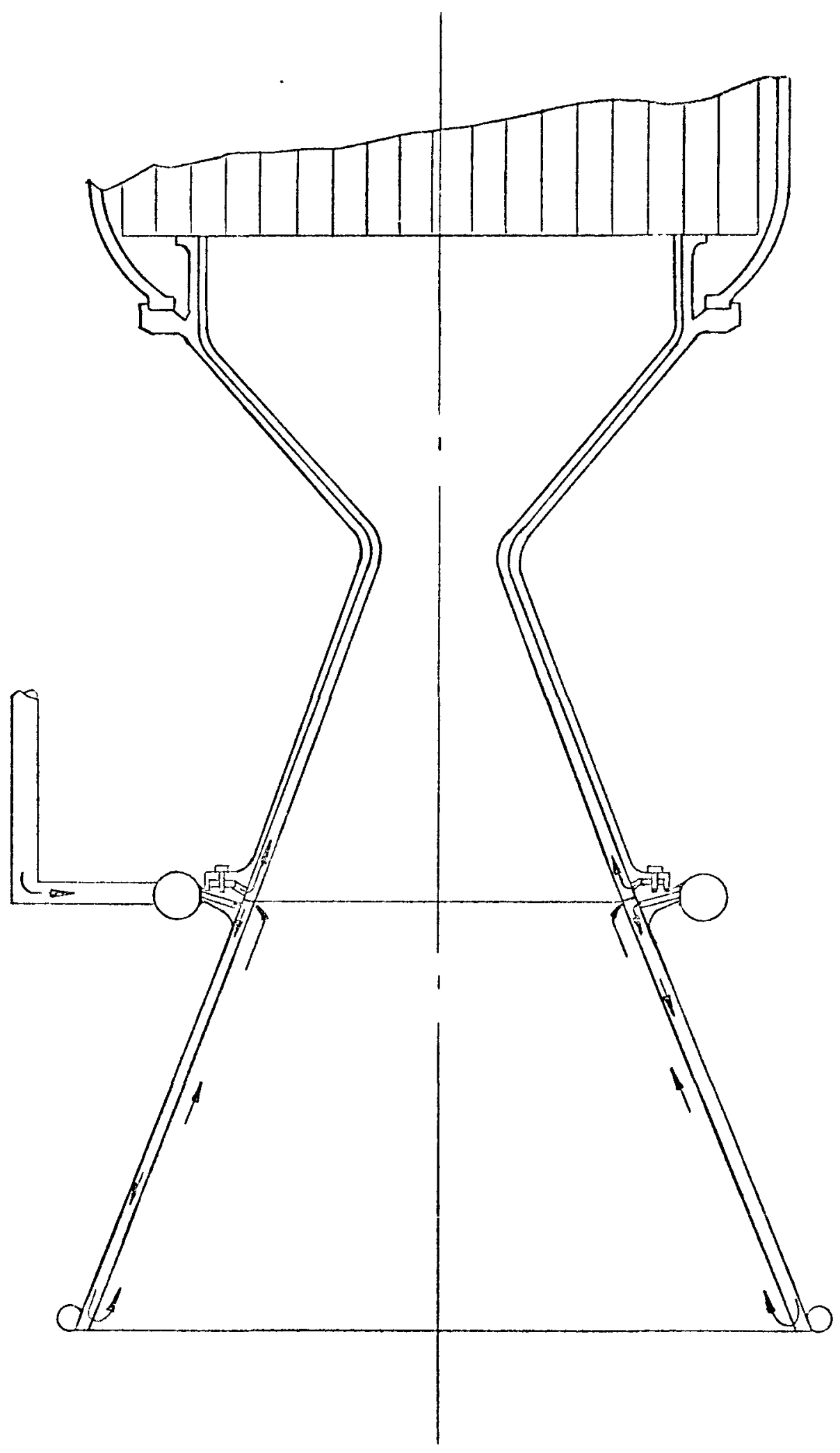

FIG. I3 


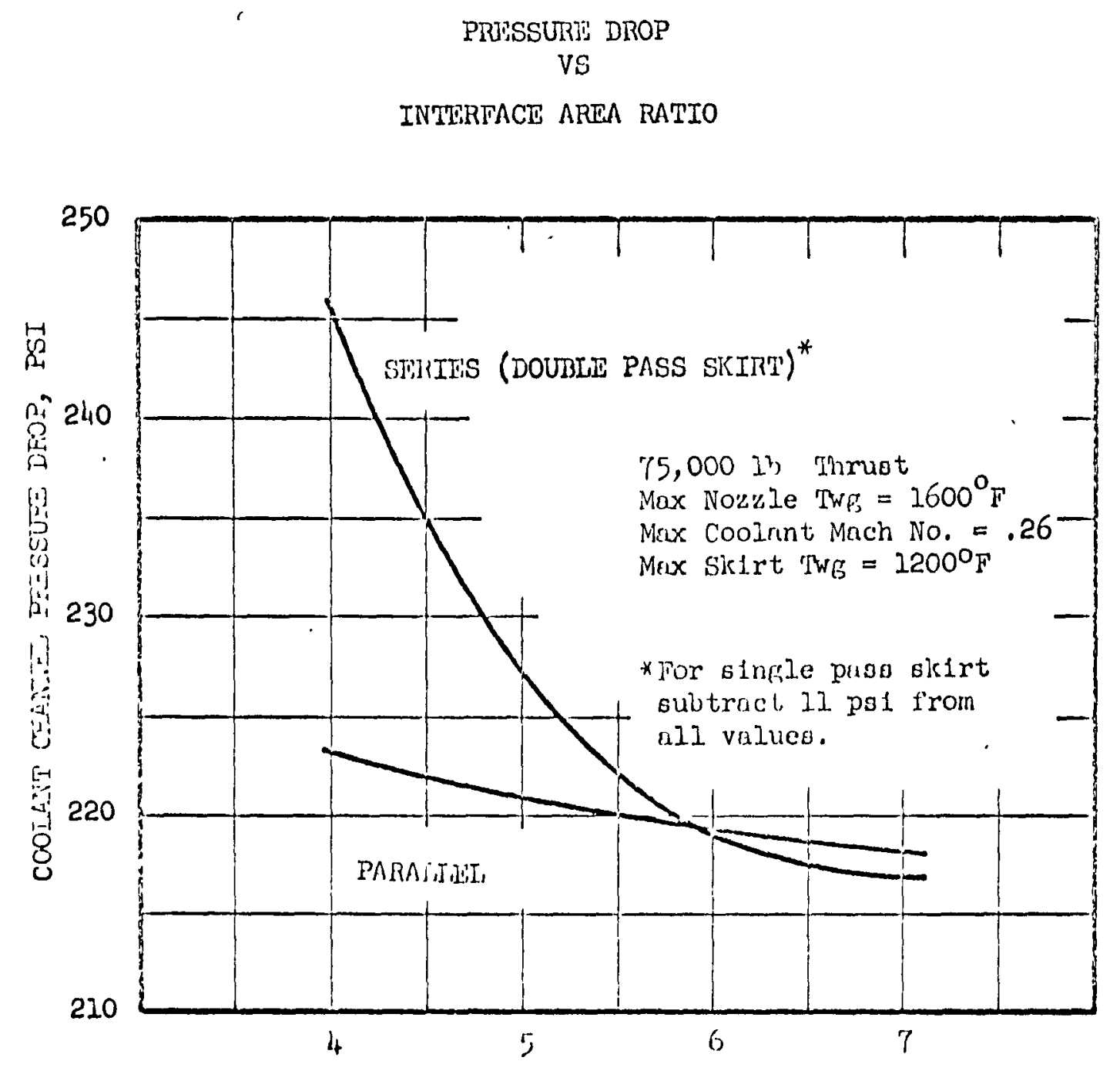

NOZZLLE/SKIKI INITRFACE NREA RATIO 


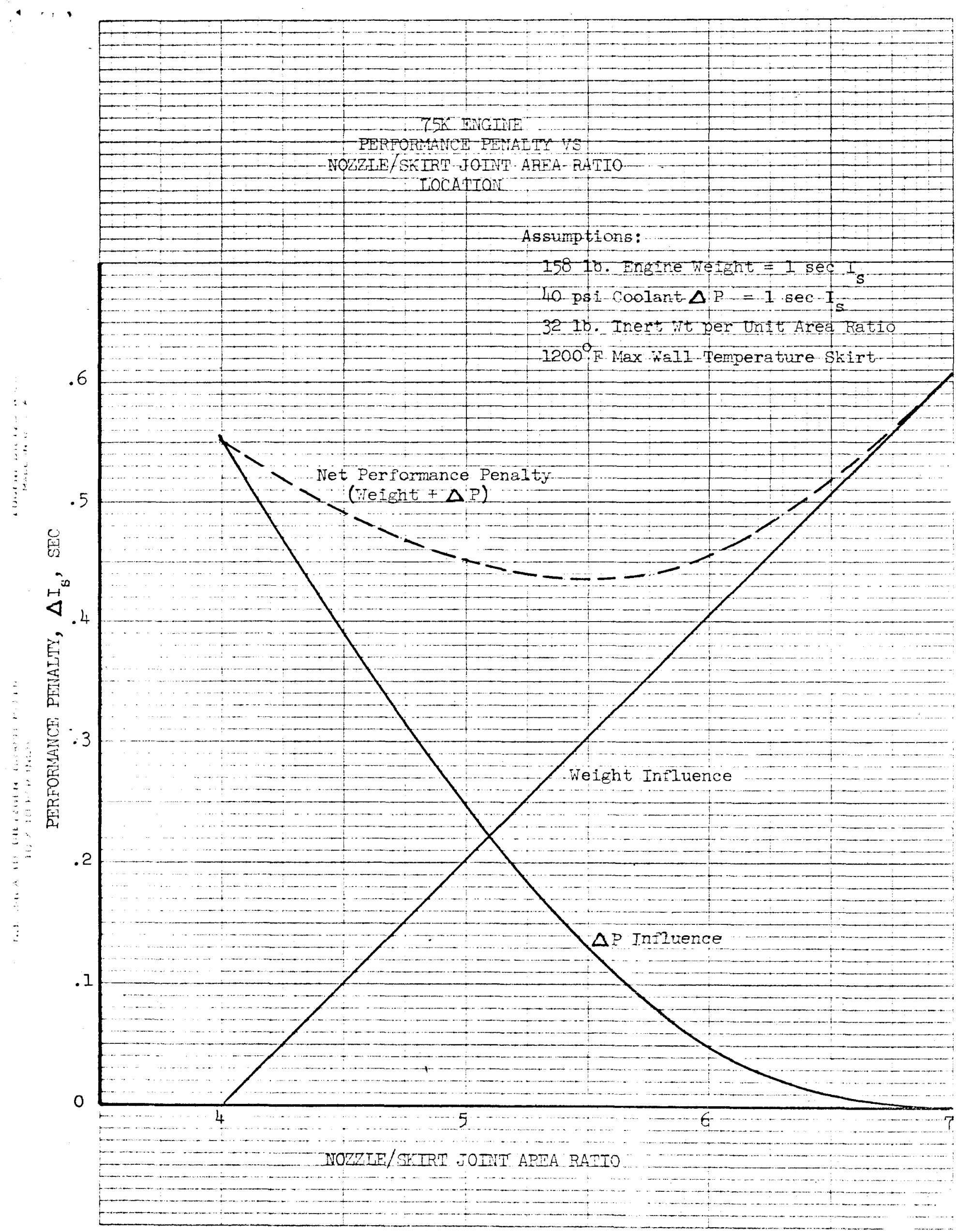




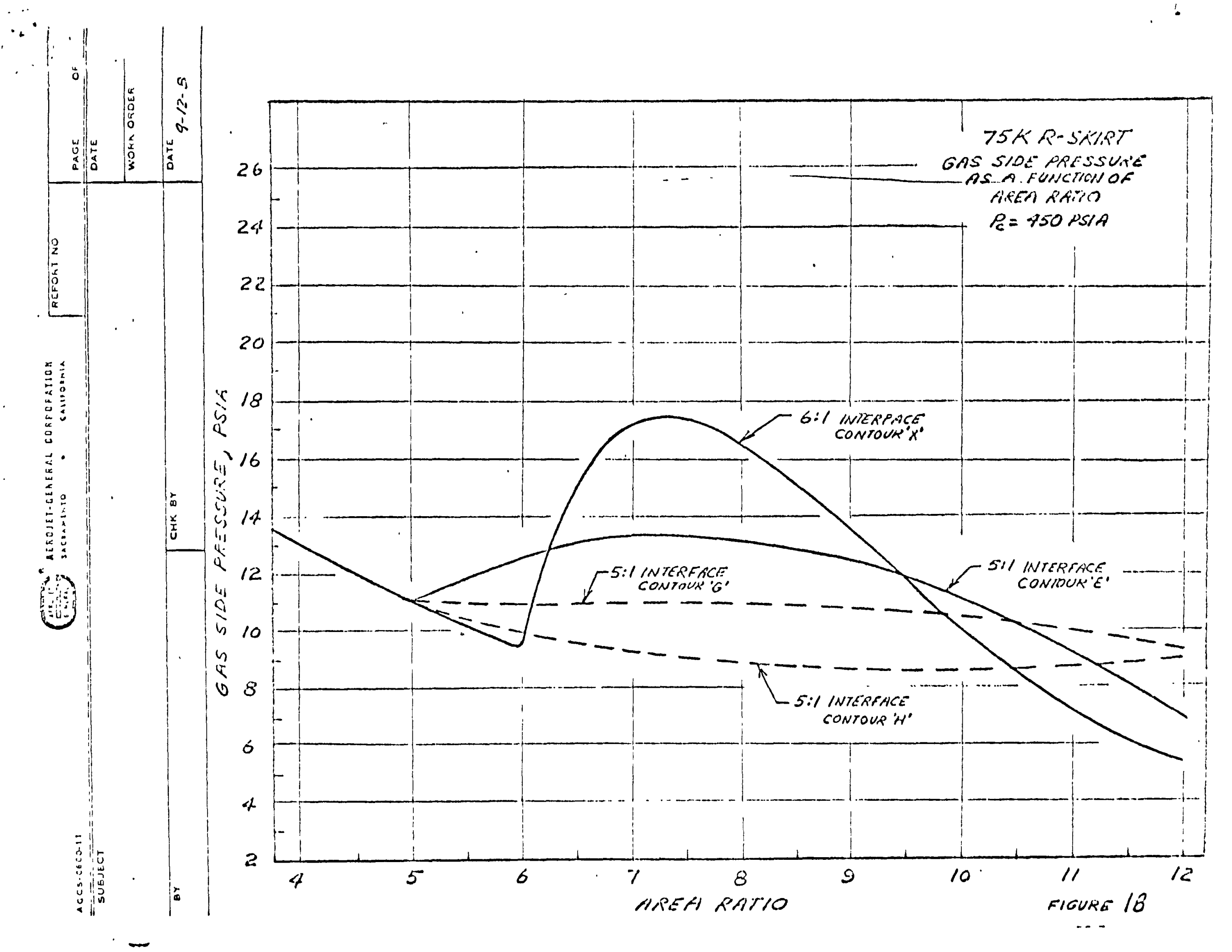




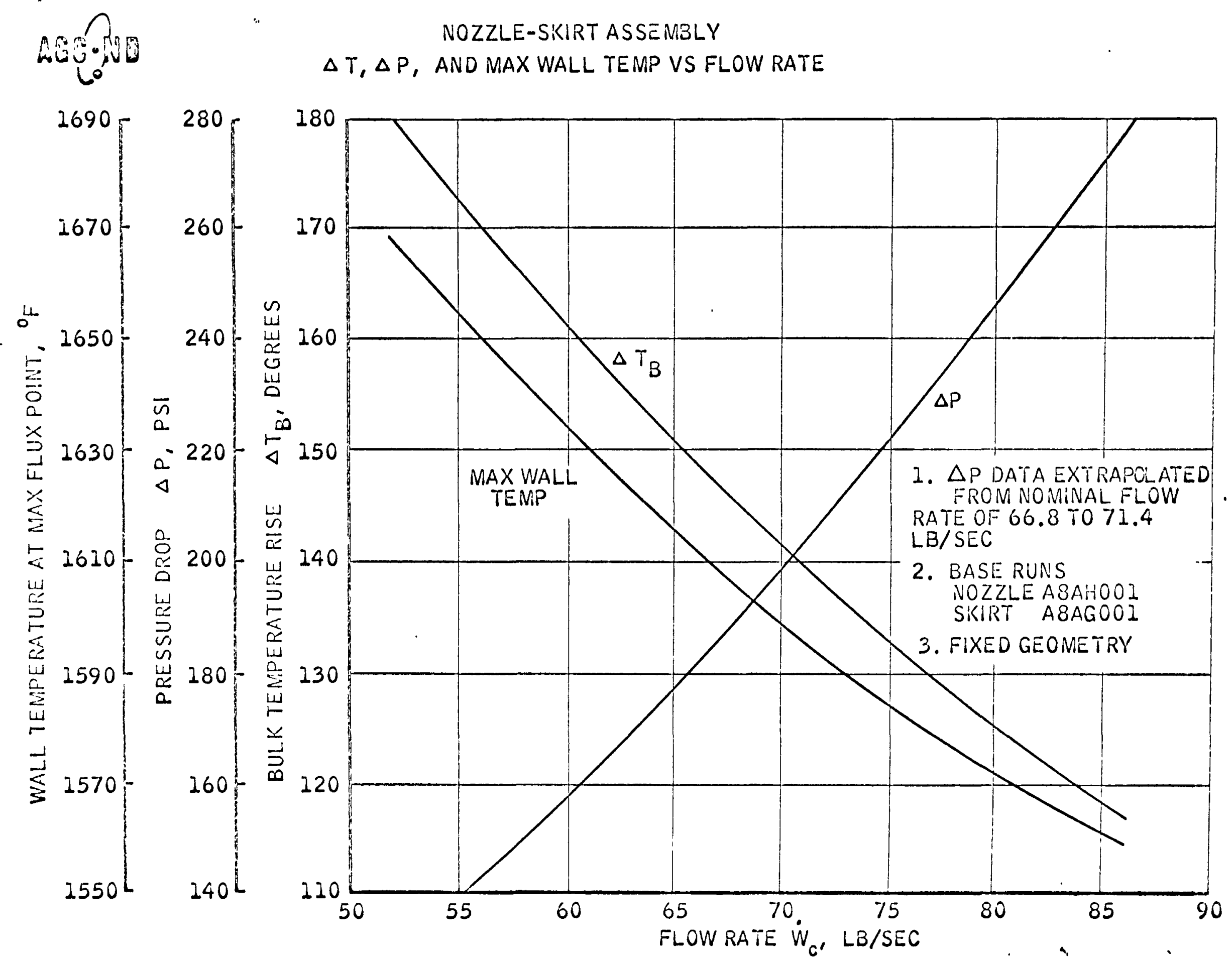




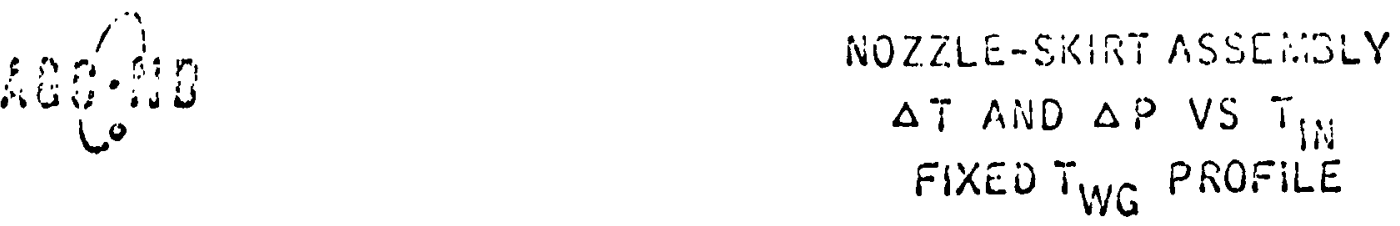

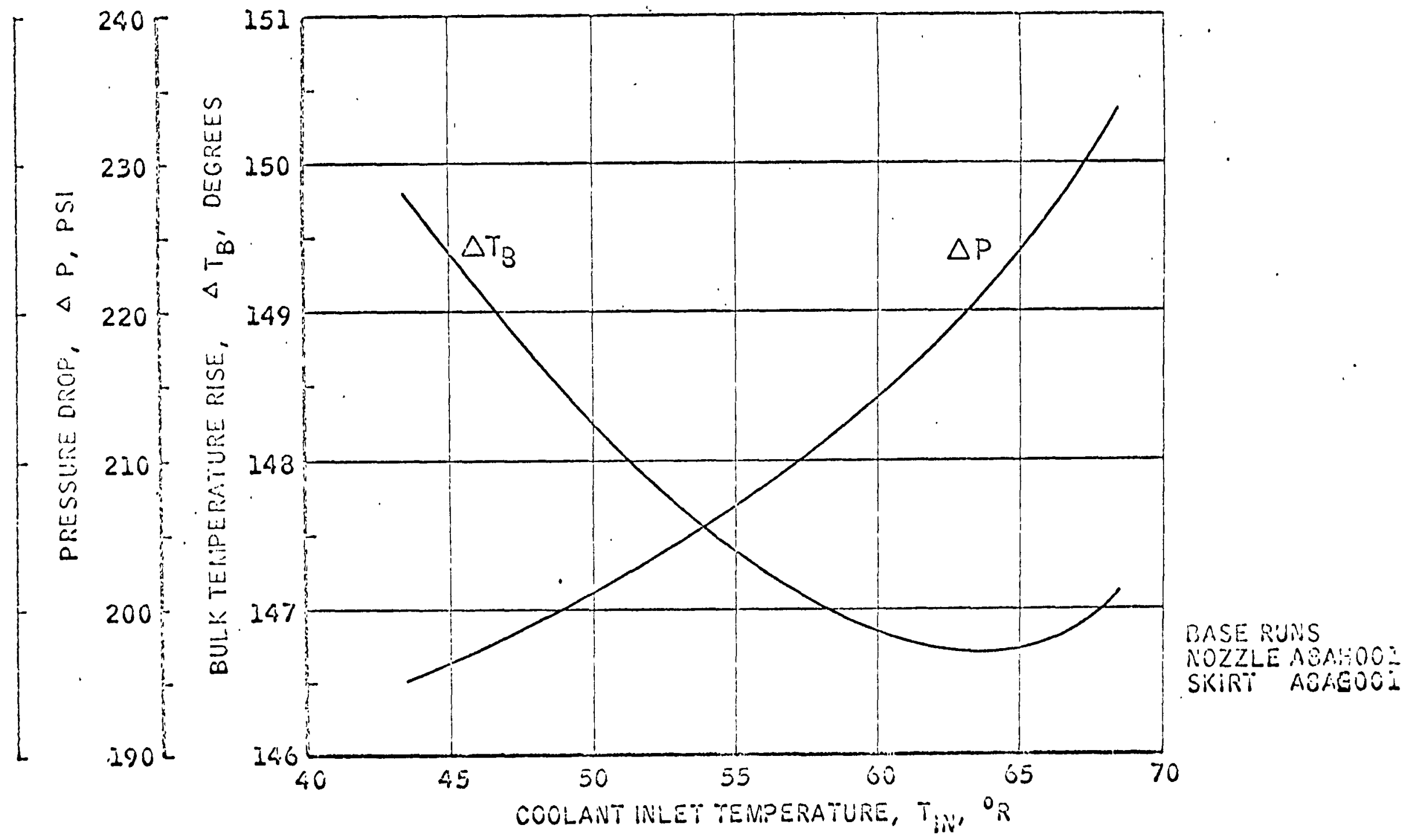




\section{NOZZLE TRADE STUDY}

PERT EVENT NO.

SCOPE OF STUDY

STATUS

RESULTS
S 1165128

DETERMINE RELATIONSHIPS BETWEEN VARIATIONS IN NOZZLE CONTOUR AND AREA RATIO WITH RESPECT TO WEIGHT AND SPECIFIC IMPULSE

$20 \%$ COMPLETE

NOT AVAILABLE 
ace.jno

SHIFTMG EQULLUBRIUN RAO NOZZLE PERFORMANCE

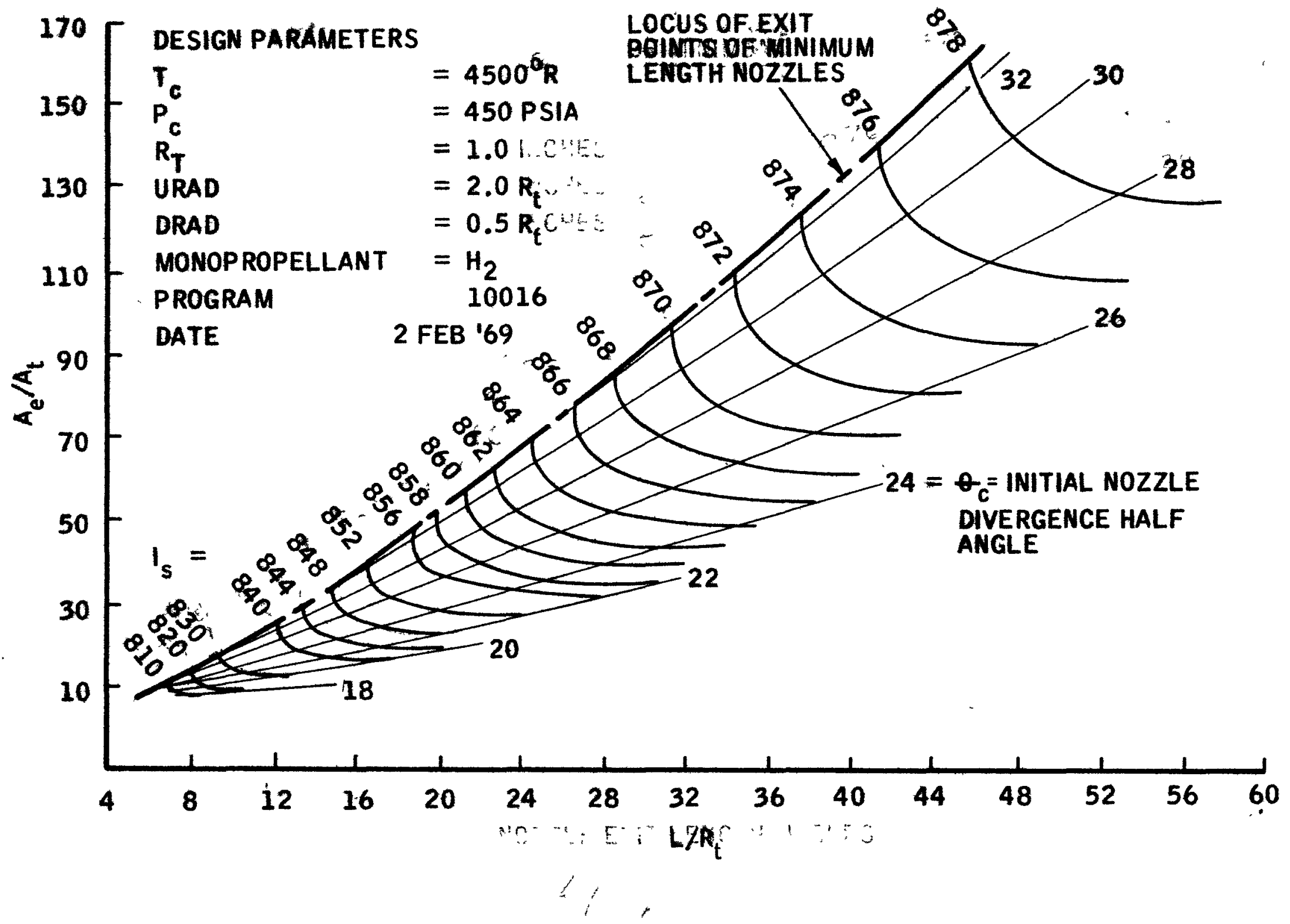


NOZZLE TRADE STUDY

PERT EVENT NO.

SCOPE OF STUDY

STATUS

RESULTS
S 1165130

DETERMINE SHIELDING REQUIREMENTS FOR VARIATIONS IN NOZZLE CONTOURS AND AREA RATIOS

SCHEDULED TO START DATE 3-10-69

NOT AVAILABLE 


\section{ABC.MO}

PERT EVENT NO.

SCOPE OF STUDY

STATUS

RESULTS

\section{NOZZLE TRADE STUDY}

S 1165132

EVALUATE PERFORMANCE PENALTIES AS A FUNCTION OF NOZZLE ASSEMBLY AND SHIELD WEIGHTS FOR ALL MISSIONS ANDESTABLISH OPTIMUM SHIELD/NOZZLE ASSEMBLY FOR EACH MISSION

SCHEDULED TO START DATE 4-15-69

NOT AVAILABLE 


\section{AEC.9.10}

PERT EVENT NO.

SCOPE OF STUDY

STATUS

RESULTS
NOZZLE TRADE STUDY

S 1165176

ANALYSIS OF NOZZLE SYSTEM HAETERATIONS FOR VARIOUS MISSIONS

1. NOZZLE CONTOUR MODIFICATION

2. SKIRT ELIMINATION

$80 \%$ COMPLETE

NOTIAVAILABLE 


\section{NOZZLE TRADE STUOY}

PERT EVENT NO.

SCOPE OF STUDY

STATUS

RESULTS
S 1165183

PRELIMINARY PERFORMANCE STUDY - USING THREE DIFFERENT BASIC NOZZLE DESIGNS, MODIFY THE CONTOUR TO EXTEND TO OTHER EXIT AREA RATIOS FOR VARIOUS MISSION APPLICATIONS AND DETERMINE PERFORMANCE DEGRADATION

COMPLETED

PRELIMINARY EXAMINATION DID NOT REVEAL CONSISTENT PERFORMANCE DEGRADATION. ANALYTICAL METHOD REVISED 


\section{NOZZLE TRADE STUDY}

PERT EVENT No.

SCOPE OF STUDY

STATUS

RESULTS
S 1165186

REFINED PERFORMANCE STUDY - A MORE RIGOROUS ANALYSIS TO DETERMINE ACTUAL PERFORMANCE VALUES ( $I_{S P}$ ) WHEN USING THREE DIFFERENT BASIC NOZRLE DESIGNS WITH MODIFIED CONTOURS EXTENDING TO OTHER EXIT AREA RATIOS FOR VARIOUS MISSION APPLICATIONS

$25 \%$ COMPLETE

NOT AVAILBBLE 
PERT EVENT NO.

SCOPE OF STUDY

STATUS

RESULTS
S1165177

SKIRT ELIMINATION ANALYSIS - REPLACE CRYO-COOLED SKIRT BY EXTENDING THE GRAPHITE SKIRT EXTENSION TO THE NOZZLE

COMPLETE

ESTABLI SHED FEASIBILITY OF REPLACING CRYO-COOLED SKIRT BY EXTENDING THE GRAPHITE SKIRT EXTENSION TO THE NOZZLE 


\section{NOZZLE TRADE STUDY}

PERT EVENT NO.

SCOPE OF STUDY

STATUS

RESULTS
S1165178

NOZZLE COOLANT STUDY - NOZZLE COOLANT TEMPERATURE, PRESSURE AND MACH NUMBER EFFECTS AS A FUNCTION OF NOZZLE/GRAPHITE SKIRT EXTENSION INTERFACE

COMPLETE

GRAPHICAL RESULTS TO BE PREPARED 
$\triangle P$ AND $\triangle T_{B}$ AND THROAT MACH NUMBER FOR VARIED COOLANT INLET AREA RATIO

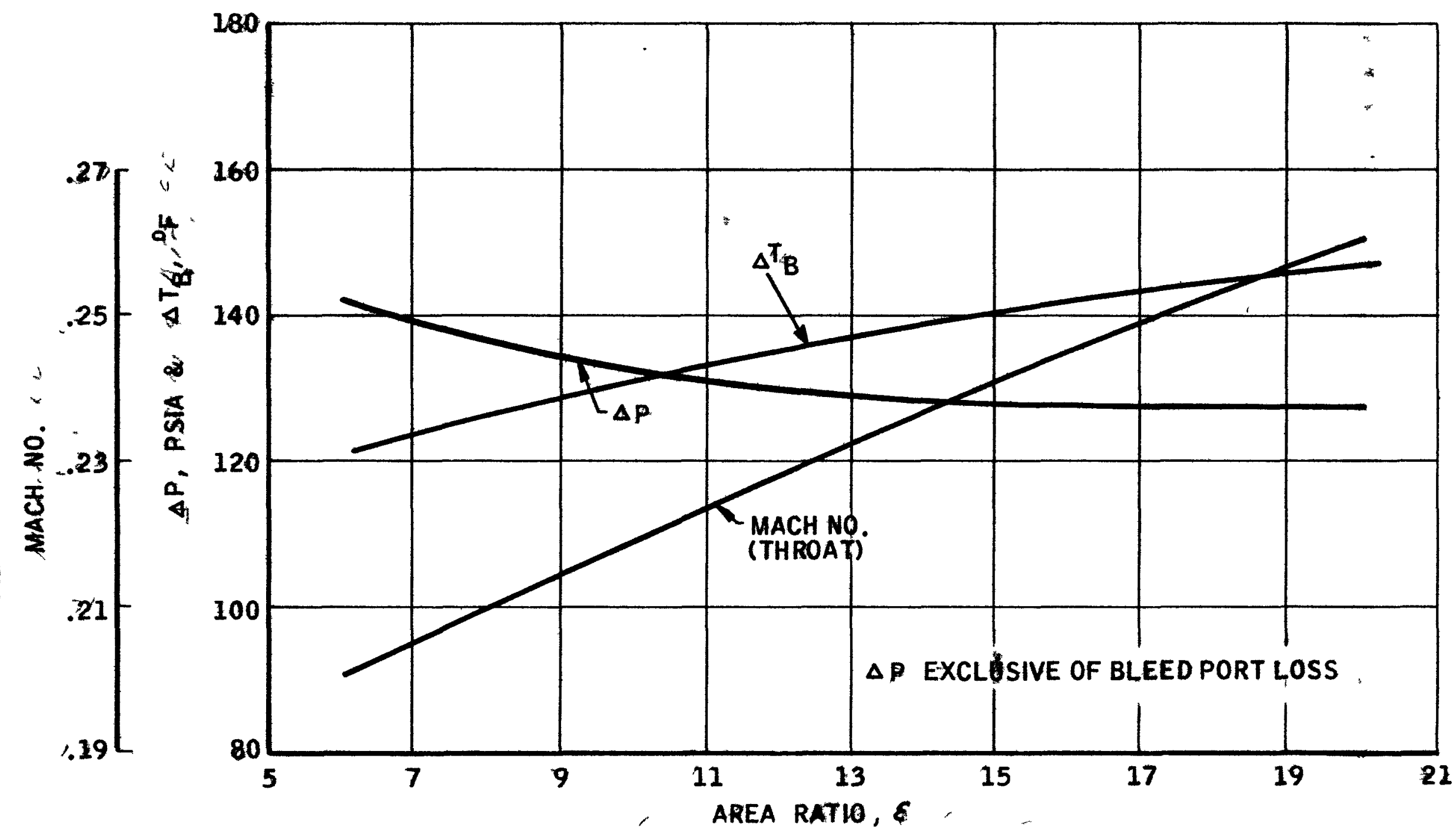


NOZZLE TRADE STUDY

PERT EVENT NO.

SCOPE OF STUDY

STATUS

RESULTS
S1165179

THERMAL ANALYSIS OF FIBER REINFORCED GRAPHITE SKIRT EXTENSION

COMPLETE

GRAPHICAL RESULTS TO BE PREPARED 
RADIATION COOLED GRAPHITE SKIRT EXTENSION

INSIDE WALL TEMPERATURE AS A FUNCTION OF AREA RATIO

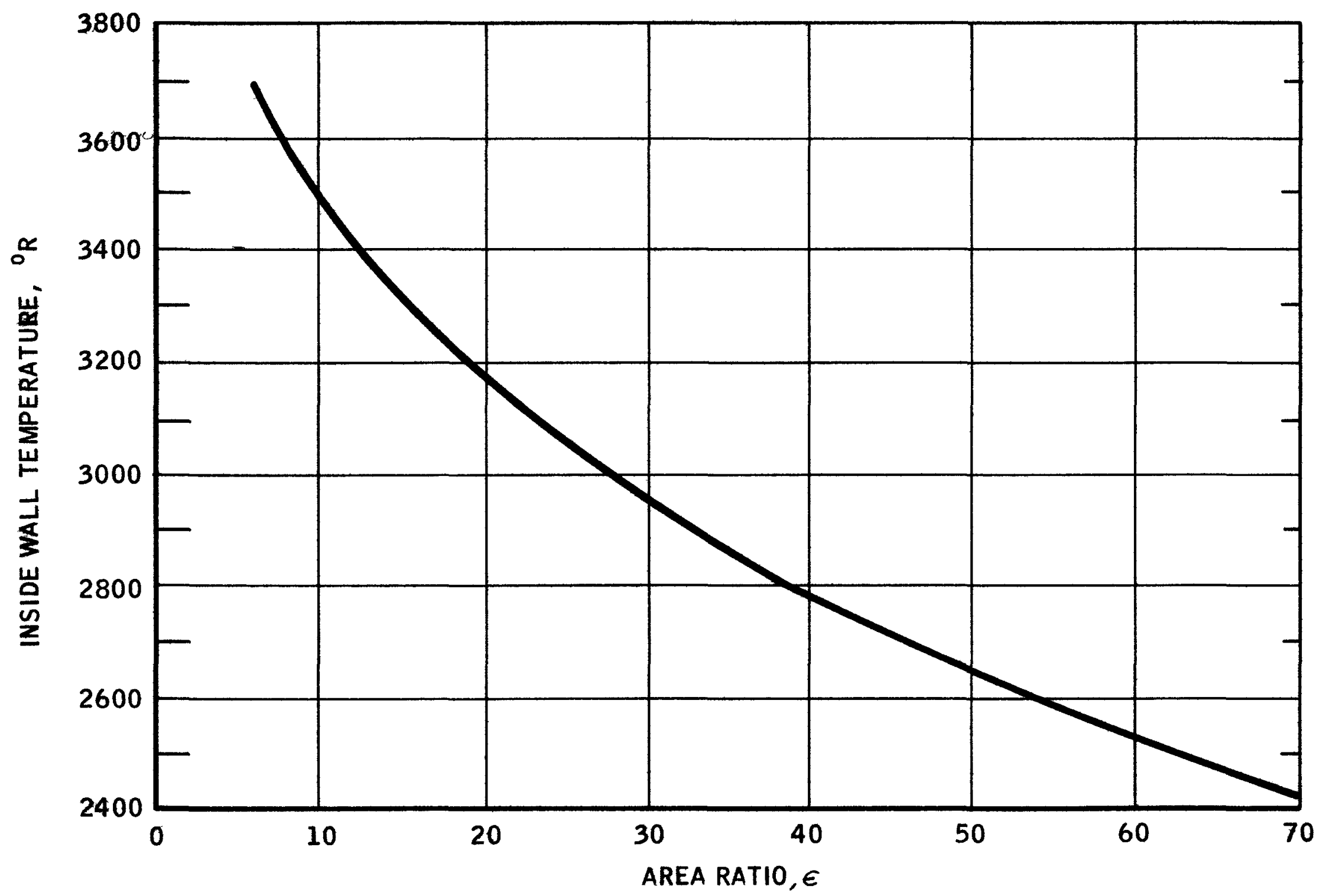




\section{NOZZLE TRADE STUDY}

PERT EVENT NO.

SCOPE OF STUDY

STATUS

RESULTS
S 1165101

MATERIALS INVESTIGATION FOR RADIATION COOLED SKIRT EXTENSION. MATERIAL CANDIDATES MUST EXHIBIT LOWER SECONDARY GAMMA EMIS SION THAN REFERENCE ENGINE SKIRT EXTENSION.

STUDY COMPLETED

CARBON AND GRAPHITE WERE FOUND TO BE THE ONLY MATERIALS CAPABLE OF MEETING THE REQUIREMENTS OF THE STUDY. FURTHER STUDY WILL BE MADE ON METALLICS AS BACKUP TO GRAPHITE. 


\section{NOZZLE TRADE STUDÝ}

PERT EVENT No.

SCOPE OF STUDY

STATUS

RESULTS

REFRACTORY ALLOY

MOBHU ALLOYS

TANTALUM ALLOYS

MOLYBDENUM

TUNGSTEN

TUNGSTEN - 25 RHENUM *

$S 1165118$

LITERATURE SEARCH FOR METALS CAPABLE OF USE ON SKIRT EXTENSION WITH RADIATION COOLING ONLY

IN PROCESS;, COMPLETION SCHEDULED FOR 5-15-69

PRELIMINARY

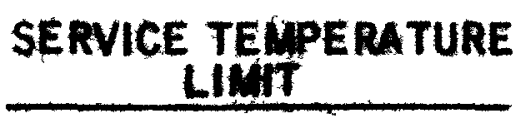

$2700^{\circ} \mathrm{R}$

$3100^{\circ} \mathrm{R}$

$3100^{\circ} \mathrm{R}$

$3300^{\circ} \mathrm{R}$

$3350^{\circ} \mathrm{R}$
LOW TEMPERATURE DUCTILITY 2WD CYCLE

GOOD

GOOD

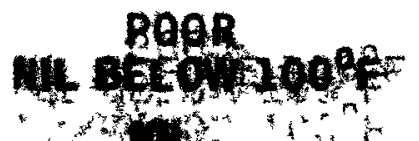

5-10\% RLIOAC.
GH $_{2}$ GMnETHIT

EMBRITTLED

EABRITTLED

UNAFFECTED

UNAFFECTED

UNAFFECTED

* NOTE: AVAILABLE ONLY IN NARROW SHEET, HIGH MATERIAL \& FAB COOST 


\section{NOZZLE TRADESTUOY}

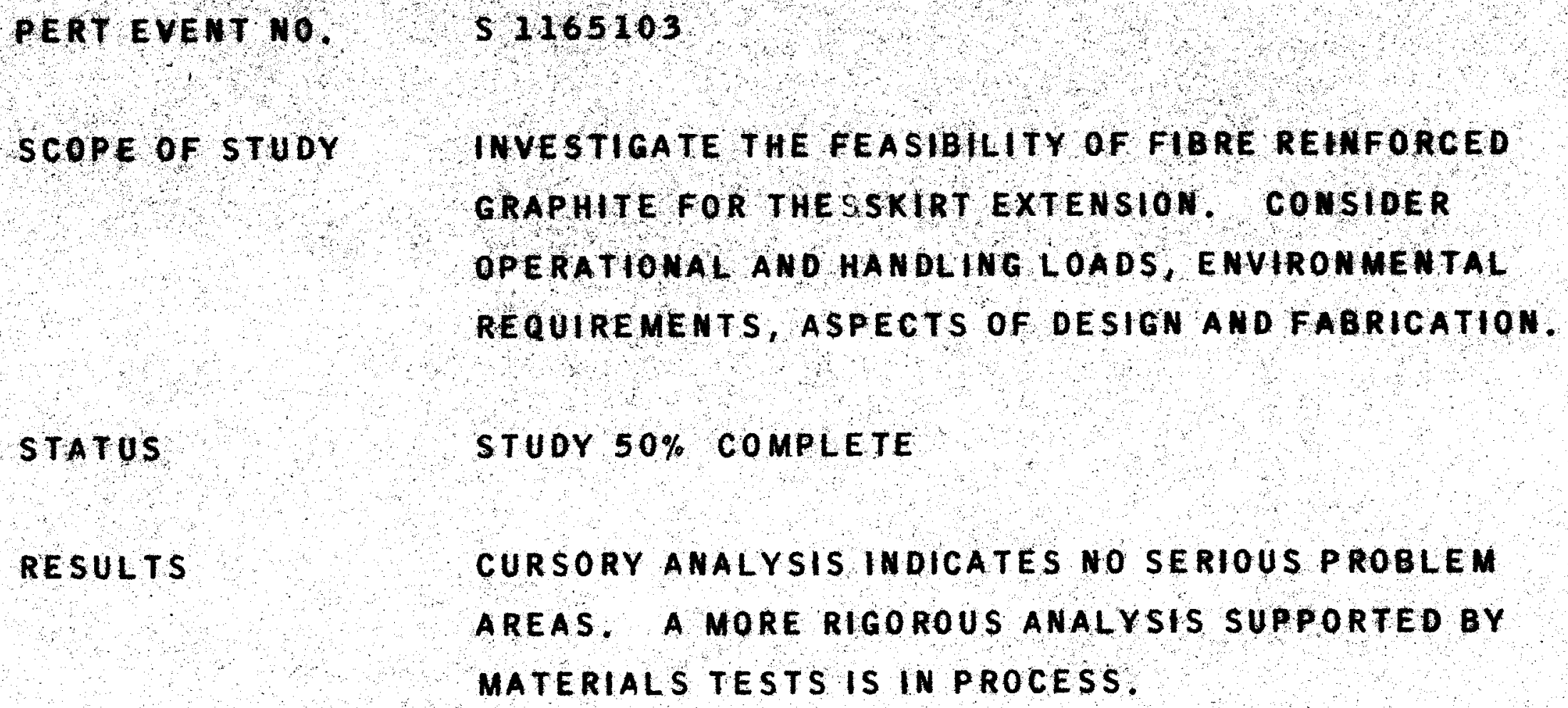


PERT EVENT NO.

SCOPE OF STUDY

STATUS

RESULTS

\section{4}

WWUSTRY SEARCH FOR MECHANICAL AHO PHYSICAL PROPERTIES DATA AHD FABRICATION TECHNIQUES AND PROCESSES FOR FIBRE REIMFORCED COMPOSITES OF GRAPHITE ANB CARBON.

PROOBOE CONCEPTUAL DESIGNS OF SKIRT EXTENSION FROM THIS INFORMATION

TO BE COMPLETED $3-15-69$

DATA ON UNION CARBIDE CORP AND THE CARBORUNDUM COMPANY PRODUCTS (TRADE NAMES: THORMEL AND CARBITEX) ARE BEING ASSEMBLED.

FABRICATION TECHNIQUES; TAPE WRAP, SHIMGLE WRAP, FILAMENT WINDING, AND INT EGRATED HONEYCOMB REINFORCEMENT (AGC TRADE NAME; INTREMOLD) ARE BEING ASSESSED. 

NOZZLE TRADE STUDY

PERT EVENT NO.

SCOPE OF STUDY

STATUS

RESULTS

\section{$\$ 1165106$}

PREIMINARY STRESS ANALYSIS OF FIBRE REINFORCED GRAPHITE SKIRT EXIENSION TO IDENTIFY FAILURE MODES AND MECHANICAL DESIGN REQUIREMENIS. THIS ANALYSIS IS LIMITED IN EFFECTIVENESS BY THE RUDIMEETARY NATURE OF MATERIAL PROPERTIES AVAILABLE.

STUDY COMPLETED

A SKIRT EXTENSION WITH AN AVERAGE THICKNESS GREATER THAN 0.2 IN. SHOULD BE ADERUATE FOR ALL STATIC AND ACCEERATION LOADS. THE EFFECTS OF VIBRATION EXCITATION CANNOT BE ADEQUATELY DETERMINED UNTIL BETTER DATA IS AVAILABLE. 
PERT EVENT NO.

SCOPE OF STUDY

STATUS

RESULTS

\section{C1165108}

PROCURE FIBRE REINFORCED GRAPHITE COMPOSITE MATERIAL FOR MECHANICAL AND PHYSICAL PROPERTIES INVESTIGATION. THE DATA TO BE USED TO REFINE THE STRUCTURAL ANALYSIS, ANO TO EVALUATE SOME VARIATIONS IN TECHNIQUES OF FABRICATION.

FIRST SHIPMENT IN TRANSIT TO AGC-SACRAMENTO. EXPECT TO RECEIVE COMPLETE ORDER BY 24 MARCH 1969.

THE PRODUCT CHOSEN FOR EVALUATION IS AG CARB-101, A GRAPHITE SQUARE WEAVE CLOTH IN A GRAPHITE MATRIX. FLAT PLATES, $1 / 4^{\prime \prime}$ AND $1^{\prime \prime}$ THICK, AND CONES $1 / 2^{\prime \prime}$ THICK WERE PROCURED. RIGID CONTROL WAS MAINTAINED OF EACH STEP OF FABRICATION PROCESS AND COMPLETE DOCUMENTATION MAINIAINED. 
PERT EVENT NO.

SCOPE OF STUDY

STATUS

RESULTS

\section{NOZZLE TRADE STUDY}

$\$ 1165126$

CONDUCT MECHANICAL AND PHYSICAL PROPERTIES TESTS OF AGCCARB-101 AT ROOM TEMPERATURE AND AT TEMPERATURE ABOVE MAXIMUM EXPECT OPERATING POINT

SCHEDULED TO START 3-15-69

TEST SPECIMEN DESIGN AND CUTTING PLAN IS COMPLETE

SCHEDULE OF TESTS TO BE CONDUCTED IS COMPLETE TEST FACILITIES ARE AVAILABLE TEST FUNDS HAVE BEEN REEEASED

TABULATION OF TESTS ON NEXT VIEWGRAPH 


\begin{tabular}{|c|c|c|c|c|c|}
\hline TYPE OF TEST & TEST & NE. & TYPE OF TEST & TEST ${ }_{\text {TEMP. }}{ }^{\circ} \mathrm{F}$ & NE. \\
\hline $\begin{array}{l}\text { 1. PRELIM TENSILE } \\
\text { 2. TENSILE }\end{array}$ & $\begin{array}{l}\text { R.T. } \\
\text { R.T. } \\
2000 \\
3000\end{array}$ & $\begin{array}{l}80(80) \\
46(48) \\
35(30) \\
35(30)\end{array}$ & $\begin{array}{l}\text { 10. FATIGUE } 10^{3} \text { to } 10^{6} \text { CYCLES } \\
\text { 11. ELECT. RESISTIVITY }\end{array}$ & $\begin{array}{l}\text { R.T. } \\
1500 \\
2000 \\
\text { R.T. }\end{array}$ & $\begin{array}{l}36(35) \\
34(0) \\
0(35) \\
5(10)\end{array}$ \\
\hline $\begin{array}{l}\text { 3. HOOP } \\
\text { 4. BIAS TENSILE }\end{array}$ & $\begin{array}{l}\text { R.T. } \\
2000 \\
\text { R.T. } \\
2000 \\
3000\end{array}$ & $\begin{array}{c}14(9) \\
0(6) \\
10(10) \\
0(10) \\
10(0)\end{array}$ & $\begin{array}{l}\text { 12. THERMAL EXPANSION } \\
\text { 13. FRACTURE TOUGHNESS }\end{array}$ & $\begin{array}{l}\text { R.T. to } \\
1500 \\
\text { R.T.T to } \\
3000 \\
\text { R.T. }\end{array}$ & $\begin{array}{l}6(0) \\
6(6) \\
48(48)\end{array}$ \\
\hline 5. BLOCK TENSILE & R.T. & $30(30)$ & 14. HARDNESS & R.T. & $30(30)$ \\
\hline 6. COMPRESSION & $\begin{array}{l}\text { R.T. } \\
3000\end{array}$ & $\begin{array}{r}22(30) \\
8(0)\end{array}$ & $\begin{array}{l}\text { 15. POROSITY } \\
\text { 16. DENSITY }\end{array}$ & $\begin{array}{l}\text { R.T. } \\
\text { R.T. }\end{array}$ & $\begin{array}{l}3(3) \\
3(3)\end{array}$ \\
\hline & & $32(30)$ & 17. DYNAMIC MODULUS \& & R.T. to & $6(6)$ \\
\hline $\begin{array}{l}\text { 8. FLEXURAL PARALLEL } \\
\text { TO PLIES }\end{array}$ & $\begin{array}{l}\text { R.T. } \\
2000 \\
3000\end{array}$ & $\begin{array}{l}20(40) \\
20(40) \\
20(0)\end{array}$ & $\begin{array}{l}\text { DAMPING FACTOR } \\
\text { 18. PERMEABILITY }\end{array}$ & 2000 & $3(0)$ \\
\hline $\begin{array}{l}\text { 9. THERMAL DIFFUSIVITY } \\
\theta \mathrm{a} . \text { THERMAL CONDUCTIVITY }\end{array}$ & $\begin{array}{l}\text { R.T. to } \\
1500 \\
1500 \text { to } \\
3000\end{array}$ & $6(0)$ & & & \\
\hline
\end{tabular}




\section{PROPERTIES TEST PLAN \\ AG CARB- 101} TEST ${ }^{\circ} \quad$ TESTS

TVPE OF TEST

\section{TVE OF TEST}

1. PRELIM TENSILE

A.t.

2. TENSILE

R.T. 3000

3. HOOP

4. BIAS TENSILE

5. BLOCK TENSILE

6. COMPRESSION

7. INTERLAM. SHEAR

8. FLEXURAL PARALLEL R.T. TO PLIES
2000

2000

R.T.

R.T.

R.T.

2000
80

48
30
6

10
10

30

30

14. HARDNESS

30

15. BOROSTTY

40
40

9. THERMAL DIFFUSNITY

10. FATIGUE $10^{3}$ to $10^{6}$ DYCLES

11. ELECT. RESISTIVITV

12. THERMAL EXPANSION

13. FRACTURE TOUGHNESS

17. DYNAMIC MODULUS
\& DAMPING FACTOR

TEST

TENP, OF

R. 1,10 3000

RT

2000

R.T. TO 3000

R.T TO

3000

R.T.

R.T.

R.T.

R.T.

R.T. 2000
35

6

48

No.

TESTS

6

35

10

30

3

3

6 
PERT EVENT NO.

SCOPE OF STUDY

STATUS

RESULTS

NOTE
$\$ 1165124$

CONDUGT TESTS OF FIBRE REINFORCED GRAPHITE COMPOSITE TO EVALUATE EROSIONICORROSION CHARACTERISTICS WHEN EXPOSED TO A HIGH TEMPERATURE, HIGH VEIOCITY, LOW PRESSURE HYOROGEN STREAM SIMULATING THE NERVA ENGTNE EXHAUST

SCHEOULED TO START 3-15-69

TEST PLAN AND SPECIAL TEST EQUIPMENT DESIGN ARE IN PROCESS. TEST FACILITY IS AVALLABLE IHYPERSONIC PLASMA JET IN VACUUM TANK)

EXTRAPOLATED DATA FROM OTHER EXPERIMENTERS INDICATE THE REGRESSION RATE AT DESIGN CONDITIONS TO BE ON THE ORDER OF A FEW MICROINCHES PER HOUR. 
PE: EVENT NO.

SCOPE OF STUDY

STATUS

RESULTS
NOZZLE TRADE STUDY

S1165121

EVALUATE THE RESULTS OF MATERIALS PROPERTIES TESTS

OF AG CARB-10I AND GOMPARE WITH DATA AVAILABLE FROM OTHER SUPPLIERS. ASSESS THE EFFECTS OF FABRLEATION TECHNIQUES UPON THE STRUCTURAL. INTEGRITY OF THE COMPONENT AND PRODUCE A CONCEPTUAL DESIGN AND PRELIMINARY PROPERTIES SPECIFICATION. SUBMIT THESE TO STRUCTURAL ANALYSIS.

SCHEDULED TO START 5-15769

NONE AVAILABLE 
PERT EVENT NO.

SCOPE OF STUDY

STATUS

RESULTS

\section{NOZZLE TRADE STUDY}

\section{S1165113}

EVALUATE STRUCTURAL CHARACTERISTICS AND ASSESS THE REIABILITY OF THE FIBRE REINFORCED GRAPHITE SKIRT EXTENSION CONCEPT DEVEOPED FROM MATERIALS TESTS AND MATERIALS AND PROCESS INVESTIGATIONS

STUDY TO START 5-31-69

PRE IMINARY REIABILITY ASSESSMENT IS IN PROCESS, BUT NO RESULTS AVAILABLE 


\section{6-10}

PERT EVENT NO.

SCOPE OF STUDY

STATUS

RESULTS

\section{NOZZLE TRADE STUDY}

\section{$\$ 116109$}

EVALUATE REQUIREMENTS AND DEVEOP A CONCEPTUAL DESIGN FOR A DEMOUNTABLE JOINT BETWEEN A FIBRE REINFORCED GRAPHITE COMPOSITE SKIRT EXTENSION

I. AND A RUID COOLED STEE SKIRT. THE DESIGN MUST CONSIDER SEALING REQUIREMENTS.

70\% COMPLETE, SCHEDULED FOR 3-15-69

FANGE CONCEPTS HAVE BEEN PRODUCED, AND A CANDIDATE SELCTED FOR THERMAL ANALYSIS. THE RESULTS OF THE ANALYSIS WILL PROVIOE DIRECTION FOR FURTHER DESIGN CONSIDERATIONS. 


\section{NOZZLE TRADE STUDY}

PERT EVENT NO.

SCOPE OF STUDY

STATUS

RESULTS
S1165110

PERFORM HEAT TRANSFER ANALYSIS OF CANDIDATE DESIGN OF RLANGE ATIACHMENI OF FIBRE REINFORCED GRAPHITE COMPOSITE SKIRT EXTENSION TO FLUID COOLED SKIRT EXTENSION TO DETERMINE TEMPERATURE DISTRIBUTION THROUGHOUT THE JOINT.

SCHEDULED TO BE COMPLETED 2-28-69

HEAT TRANSFER MODE HAS BEEN PREPARED AND SUBJECTED TO COMPUTER PROGRAMMING. COMPUTER RESULTS ARE BEING VALIDATED AND PLOTTED. 



\section{NOZZLE TRAOE STUDY}

PERT EVENT NO.

SCOPE OF STUOY

STATUS

RESULTS
$51165138-61165139$

COMDUCT MEAT TRALSFER STUDY TO ESTABLISH MATERIAL COOLING REQUIREMGHT USING TURBINE EXHAOST GAS AS COOLANT CANDIDATE MATERIALS WERE:

$$
\begin{aligned}
& \text { CRES } 347 \\
& \text { A- } 110 \text { AT } \\
& \text { ZIRCOLOY - } 2
\end{aligned}
$$

STUDY COMPLETED

1) CRES 347 ACCEPTABLE FOR THE AVAILABLE TURBINE EXHAUST GAS CONOITION

2) A-110AT \& QIRCOLOY-2 UNACCEPTABLE

3) ATTEMPIS WERE MADE TO DILUTE THE TURBINE EXHAUST GAS TO LOWER TEMPERATURE FROM $1306^{\circ} \mathrm{R}$ TO $270^{\circ} \mathrm{R}$ BUT THE MIXED COOLANTS WERE INCAPABLE OF REDUCING COOLANT TUBE "T 6 " $=700^{\circ} \mathrm{F} @$ MAXIMUM COOLANT MACH $\#=0.4$ 
COOLANT MACH NUMBER VS. COOLANT FLOW RATE

FOR A WALL TEMPERATURE OF $700^{\circ} \mathrm{F}$

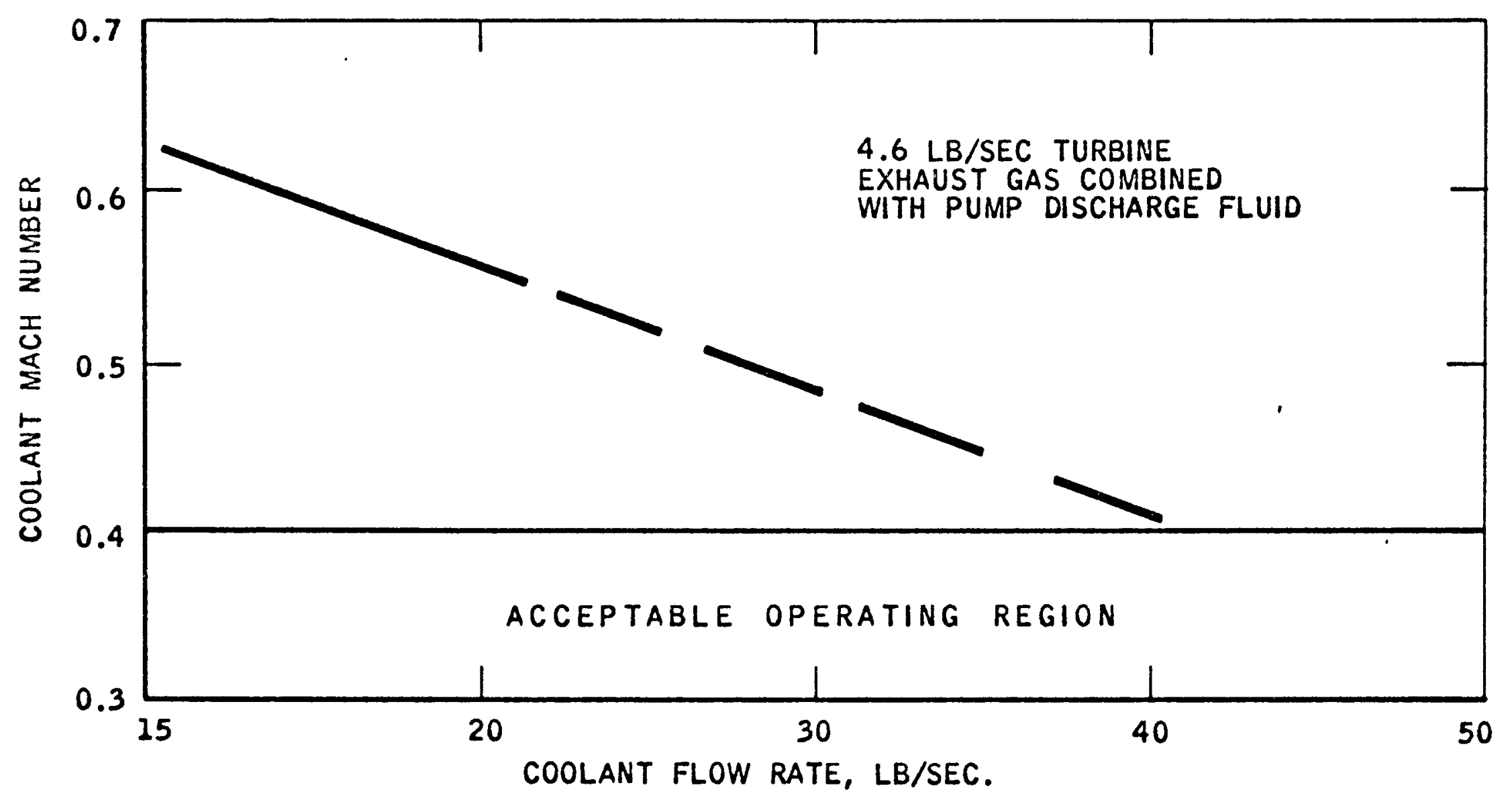


NOZZLE TRADE STUDY

PERT EVENT NO.

SCOPE OF STUDY

STATUS
$1165141-C 1165150$

CONDUCT TURBINE GAS COOLED SKIRT EXTENSION CONCEPTUAL DESIGN OF TURBINE GAS COOLED SKIRT EXTENSION CONSIST OF

MATERIAL: CRES 347

CONSTRUCTION: ONE PASS COOLANT TUBE BUNDLE BRAZED \& WITH REINFORCING HOOP BAND. FLUID ENTER AT $A_{e} / A_{t}=24: 1$ AND EXIT@ $A_{e} / A_{t}=100: 1$ THROUGH INDIVIDUAL COOLANT TUBE EXHAUST NOZZLES

PRESENT DESIGN CALLS FOR 480 COOLANT TUBE BUNDLE WITH INDIVIDUAL COOLANT TUBE EXHAUST NOZZLE (P/N 1136173)

COMPLETION SCHEDULED 15 MAY 1969 


\section{NOZZLE TRADE STUOY}

PERT EVENT NO.

SCOPE OF STUOY

STATUS

RESULTS
$51165142-C 1165151$

CONDUCT PRELIMNARY MINIMUM COOLAUT REQUIREMENT STUOY FOR REFERENCE ENGINE USING SIMGLE TURBOPUMP. DETERUINE TURBINE EXHAUST FL OW REQUIREMENT AT THE COLLECTOR OUTLET (P, T, W) FOR SKIRT EXTENSION

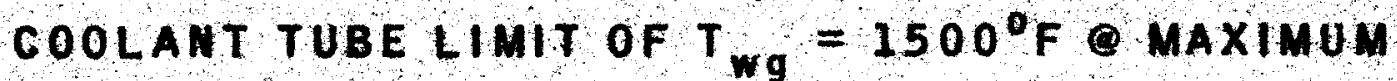
COOLANT MACH $+=0,4$

STUDY COMPLETED

GRAPHICAL DATA 


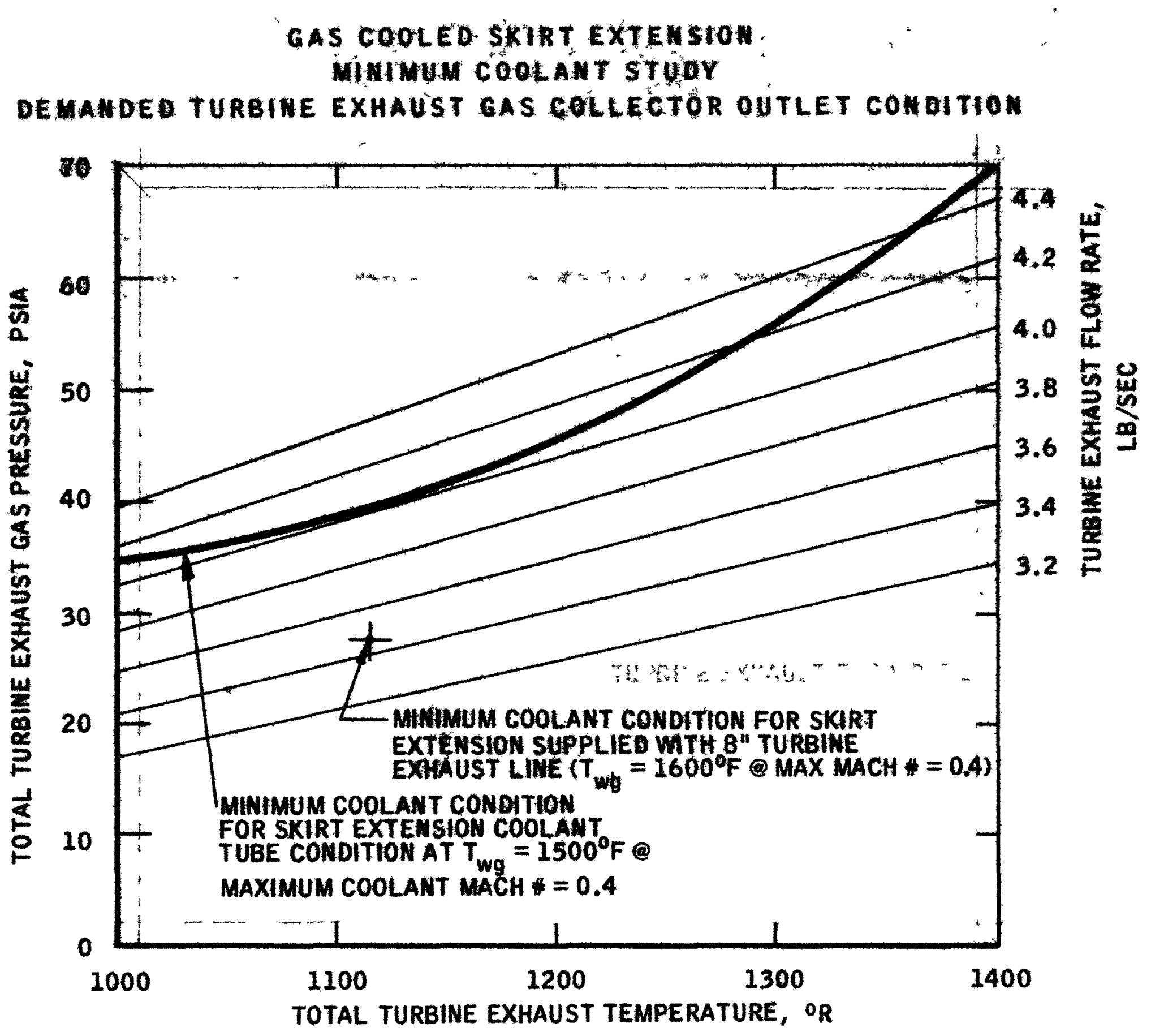


PERT EVENT NO.

SCOPE OF STUDY

STATUS
$S 1165152-C 1165153$

GAS COOLED SKIRT EXTENSION "UP-GRADE" MINIMUM COOLANT REQUIREMENTS STUDY FOR CHANGE IN STATE POINT USING TWO TURBOPUMP SYSTEM

AWATING ENGINE SYSTEM STATE POINT UTILIZING TWO TPA.

COMPLETION SCHEDULED 1 MAY 1969 


\section{MOZZLE TRADE STUDY}

PERT EVENT NO.

SCOPE OF STUDY

STATUS
S116143 - C 116140

INTERMEDIATE POWER HOLO EFFECTS ON GAS COOLED SKIRT EXTENSION DURING TRAUSIENT PHASE OF THE EUGINE SYSTEM UTILIZIMG TWO TPA \& ITERATE AS REQUIRED

AWAITING ENGNE SYSTEM DEFINITION OF INTER MEDIATE POWER LEVELS COMPLE T EON SSOHEDUL E D 1 MAY 1969 


\section{NOZZLE TRAOE STUOY}

PERT EVENT NO.

SCOPE OF STUDY

STATUS

RESULTS
$S 1165147-61165148$

CONDUCT COOLANT TUBE EXHAUST NOZZLE DESIGN/ PERFORMANCE STUOY FOR APPLICATION TO GAS COOLED SKIRT EXTENSION. DESIGN/PERFORMANCE EVALUATION OF COOLANT TUBE EXPANSION NOZZLE USING:

MOLYBDENUM

TUNGSTEN

CRES 347

COMPLETEO EXCEPT FOR DESIGN DETAILS

CRES 347 FOUND SATISFACTORY WITH INNER WALL REMOVED. 


\section{NOZZLE TRADE STUDY CRYO-COOLED, SKIRT EXTENSION}

ESTABLISH:

1. alterquitive to turbine eXhaust coOlé EXTENSION

2. BACKUP TO GRAPHITE CONSTRUCTION 


\section{NOZZLE TRADE STUDY}

PERT EVENT NO.

SCOPE OF STUDY
S1165154-C1165155

CONDUCT MATERIAL SEARCH WITH LOW CREW RADIATION DOSE FAGTOR FOR USE WITH CRYOGENIC HYDROGEN COOLED SKIRT EXTENSION OPERATING EITHER IN PARALLEI FLOW OR IN SRIES ROW WITH NOZZLEISKIRT ASSEMBLY.

THE CANDIDATE MATERIALS FOR THE GRYO-COOLED SKIRT EXTENSION CENCEPTS

CRES 347

A-HOAT (TITANIUM ALLOY)

7039761 (ALUMINUM ALLOY)

ZIRCOLOY-2 


\section{WLLLE TRADE STUOY}

PERT GVEN NO.

SCOPE OF STUDY

STATUS

RESULTS

\section{S116517 - 01165172}

MECHANICAE DESICN EVALUATION OF GRES-34, A-11OAT, 703961 MATERIAL FOR CRYO-COOLD SKTM EXTENSION APPLICATION. MECHANICAL DESIGN EVALUATION CONSISTS OF:

1) COOLANT PASSAGE DESIGN

2) FABRICABHLITY INCLUDING BRAZING AND WELIING TECHNIQUES

3) COOLANT MANIFOLD DESIGN

4) COCLANT TUBE MATERIAL THICKNESS

5) CREW RADIATION DOSE REDUCTION

STUDY COMPLETE

1) CRES-347, A-110AT, \& 7039T61 MATERIALS WERE ACCEPTABLE

2) A-1HOAT EIIMINATED ON THE BASIS OF NO ADVANTAGE OVER CRES-347 WITH RESPECT TO GENERATED SECONDARIES. 
NOZZIE COOLANT MACH INMBER

VS. COOLANT INLET \& DISCHARGE TTMMPERATURE

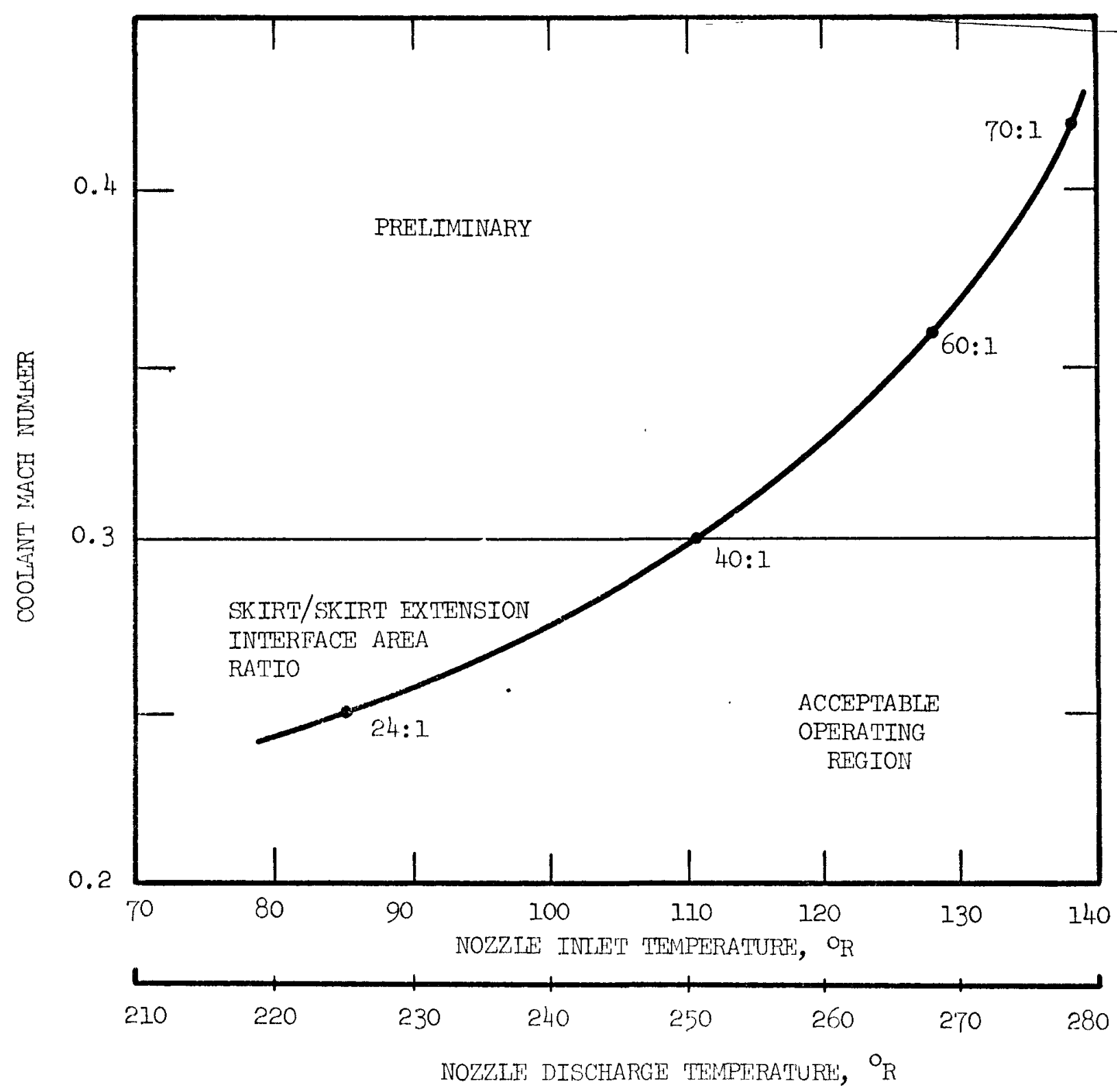




\section{NOZZLE TRROE STUDY}

PERT EVEMT NO.

SCOPE OF STUDY

CONSIDERED

RESULTS

\section{$51165156-\mathrm{CH} 65159$}

SKIRT EXIENSION CRYO-COOLING SYSTEM

1. SERIES ROW WITH NOZZZEISKIRT ASSEMBLY

2. PARALLA FLOW WITH NOZZLEISKIRT ASSEMBLY

E.IMINATED (1)

NOT ABLE TO COOL PAST $A_{e} / A_{t}=40: 1$ WITHOUT CAUSING MACH NUMBER TO EXCEED 0.3 AT THE NOZZLE THROAT 
Ace.p.

MOZZLE TRADE STUDY

PERT EVENT NO. S1165157 - C1165158

SCOPE OR STUDY: CRYO-COOLED SKIRT EXTENSION

COOLANT CIRCUIT HEAT TRANSFER STUDY

(PARALLEL FLOW)

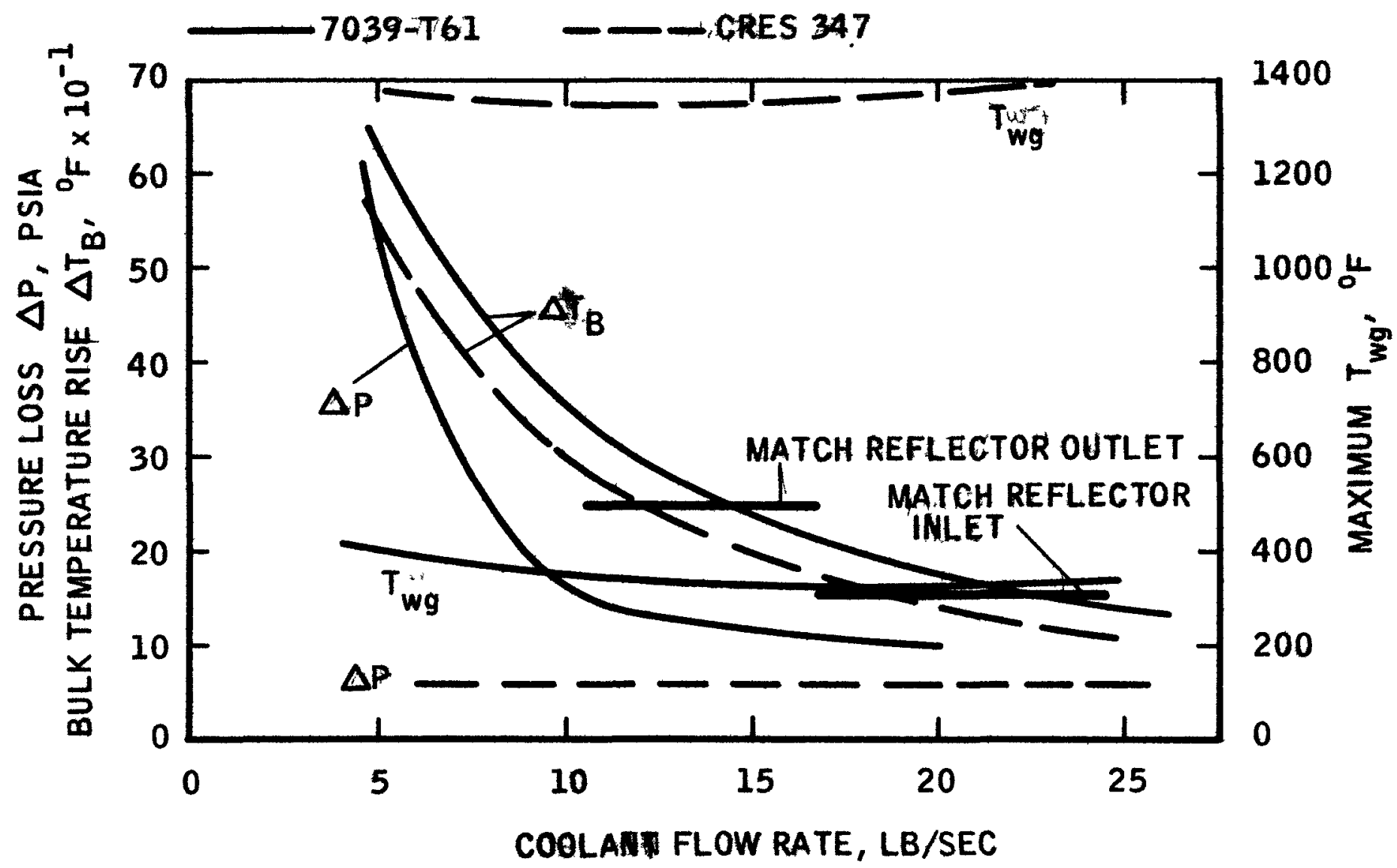




\section{NOZZLE TRABC STUDY}

PERT EVET NO.

SCOPE OF STUDY

RESULTS
$612+15-011010$

SAECTION OF CANDIOATE MATERIAL FOR CONGEPTUAL DESIGN PMASE.

CRITERIA:

1) REDUCED CREW RADIATION DOSE RATE FROM CRES 37 REFERENCE

2) EASE OF FABRICABILITY

3) COMPATIBLE WITH NOZZLE/SKIRT FLOW SCHEME

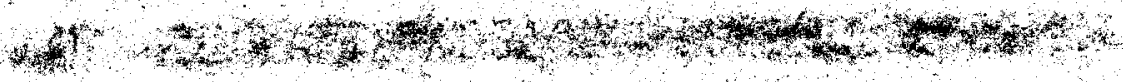

SEIECT FOR FURTHER STUDY

1) 7039T61 ACCEPTABLE FOR ABOVE CRITERIA IMAY HAVE FABRICABILITY PROBLEMS)

2) CRES-347 ACCEPTABLE AS BACK-UP FOR ABOVE CRITERIA INO REDUCTION IN CREW RADIATION DOSE RATE) 


\section{NOZZLE TRAOE STUDY}

PERT EVEM NO.

SCOPE OF STUDY

STATUS
$\$ 116162-01165175$

CONDUCT CRYO-COOLED SKIRT EXIBNSION CONCEPTUAL DESIGN STUDY

CONCEPTUAL DESIGN OF CRYO-COOLED SKIRT EXTENSION CONSISTS OF:

MATERIAL: 7039T6I ALUMINUM ALLOY CRES-347 "BACKUP" CONSTRUCTION: TWO PASS COOLANT TUBE I DOWN \& 2 UP PATTERN) BUNDLE BRAZED TOGETHER AND WITH REINFORCING HOOP BANDS AND OPERATING IN PARALLEI WITH NOZZLE/SKIRT ASSEMBLY. FLUID ENTERS \& EXITS Q $\epsilon$ - 24:1 SKIRTISKIRT EXTENSION INIERFACE.

PRESENT CONCEPT CALLS FOR 504 COOLANT TUBES WITH .012 IN. THICK WALLS (PIN 1136165) ALUMINUM 
NOZZLE TRADE, STUOY

PERT CVET NO.

SCOPE OF STUDY

STATUS

\section{$\$ 116173-0116174$}

CONDUCT FABRICATION PROCESS STUDY ON CRYO-COOLED SKIRI GKENSION MADE FROM ALUMINUM ALCOY MATERIAL

1 PRESEMTIY ANALYZING TUBE JOINING PROCESSES

2) ESTABLI SHED COOLANT TUBE WALL THICKNESS REQUIREMENT AS A FUNCTION OF REINFORCING BAND SPAGING

3) EXPECT TO COMPLETE STUDY ON I APRIL 1969

(NO EXPERIMENTAL WORK UNDERTAKENI 


\section{NOZZLE TRADE STUDY}

PERT EVENT NO.

SCOPE OF STUDY

$$
51165167-01165170
$$

CONDUCT CRYO-COOLED SKIRT EXIENSION HEAT TRANSFER STUDY

1. ESTABLISH FLOW SPLIT BETWEEN SKIRT EXTENSION \& NOZZLE/SKIRT ASSEMBLY AT INTERMEDIATE POWER HOLDS

2 DETERMINE THE DEGREE OF TEMP RISE MISMATCH

3. ANALYZE THERMAL ASPECT AT INIERMEDIATE POWER HOLDS AT $W$ AS ESTABLISHED IN (1)

4. ITERATE AS REQUIRED TO INCLUDE EFFECTS OF $3 \sigma$ ENGINE OPERATING PARAMETERS

STATUS TO BE ACCOMPLISHED 4-18-69 


\section{BOLT COOLANT \& DILUENT FLOW TRADE STUDY}

PERT EVENT S1165301-302

STATUS: COMPLETE

RESUL.TS:

METHOD OF OILUENT CONTROL

DILUENT PORT LOCATION

DCV POSITION

1. ORIFICE

DOME

2. ORIFICE

IN PV AT REFLECTOR INLET

3. ORIFICE

REVERSE BOLT FLOW PLENUM

4. DCV

DOME

BELOW SHIELD

5. DCV

IN PV AT REFLECTOR INLET

6. DCV

REVERSE BOLT FLOW PLENUM

BELOW SHIELD

7. DCV

DOME

8. DCV

IN PV AT REFLECTOR INLET

9. DCV

REVERSE BOLT FLOW PLENUM

BELOW SHIELD

ABOV E SHIELD

ABOVE SHIELD

ABOVE SHIELD 
AGC.ND

BOLT COOLANT \& DILUENT FLOW TRADE STUDY

PERT EVENT NO. S 1165311-312 (PARTIAL

FORWARD BOLT COOLANT FLOW

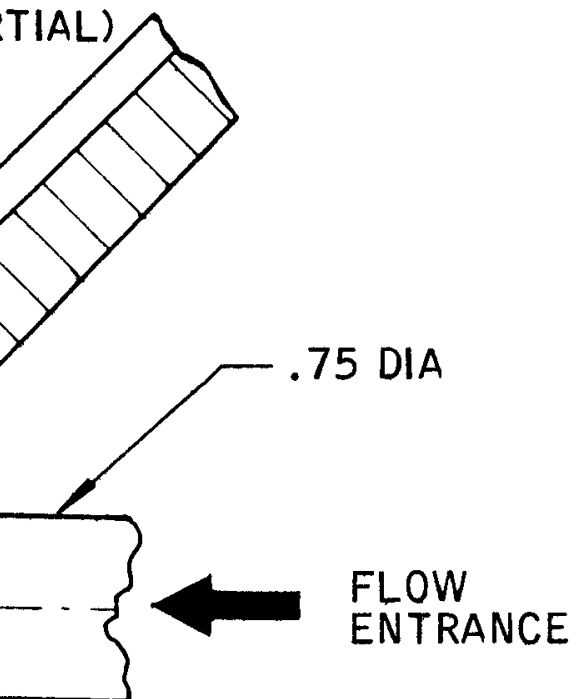

LOW

EXIT

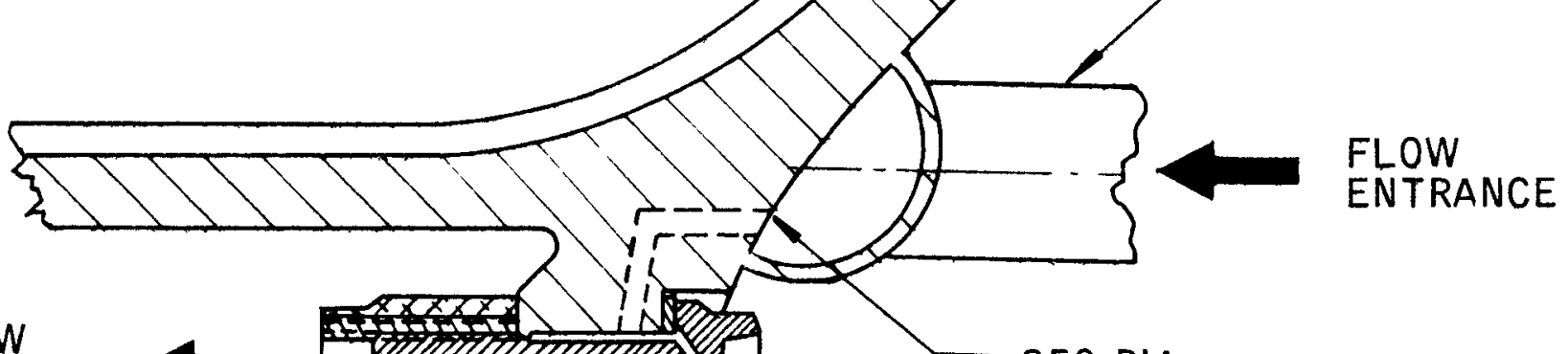

.125 DIA

.186

.192

DIA 


\section{AGC.ND}

\section{DESIGN VARIATIONS OF DILUENT PORT LOCATIONS}

IN PRESSURE VESSEI

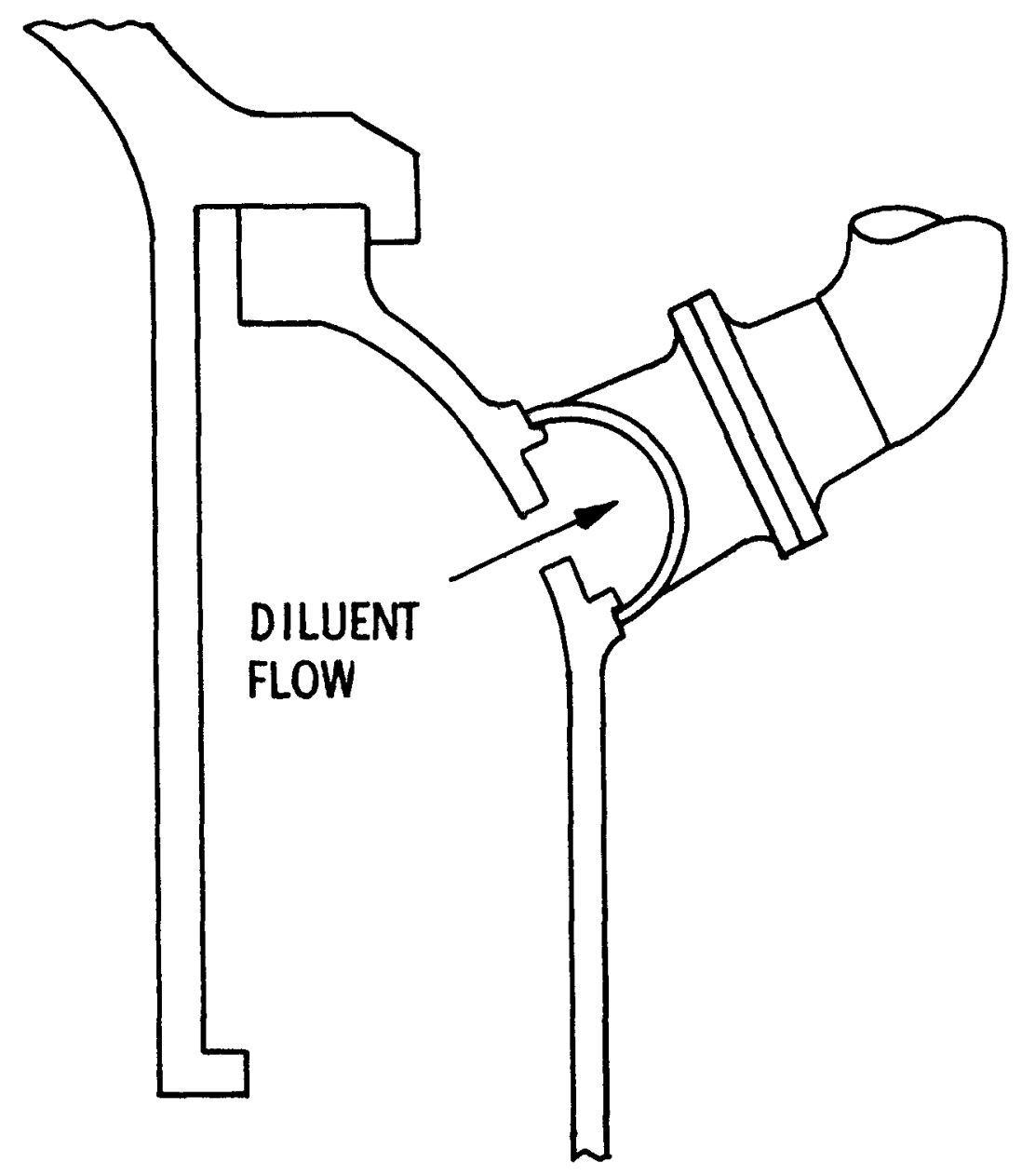

MULTIPLE PENETRATION WITH

COMMON MANIFOLD

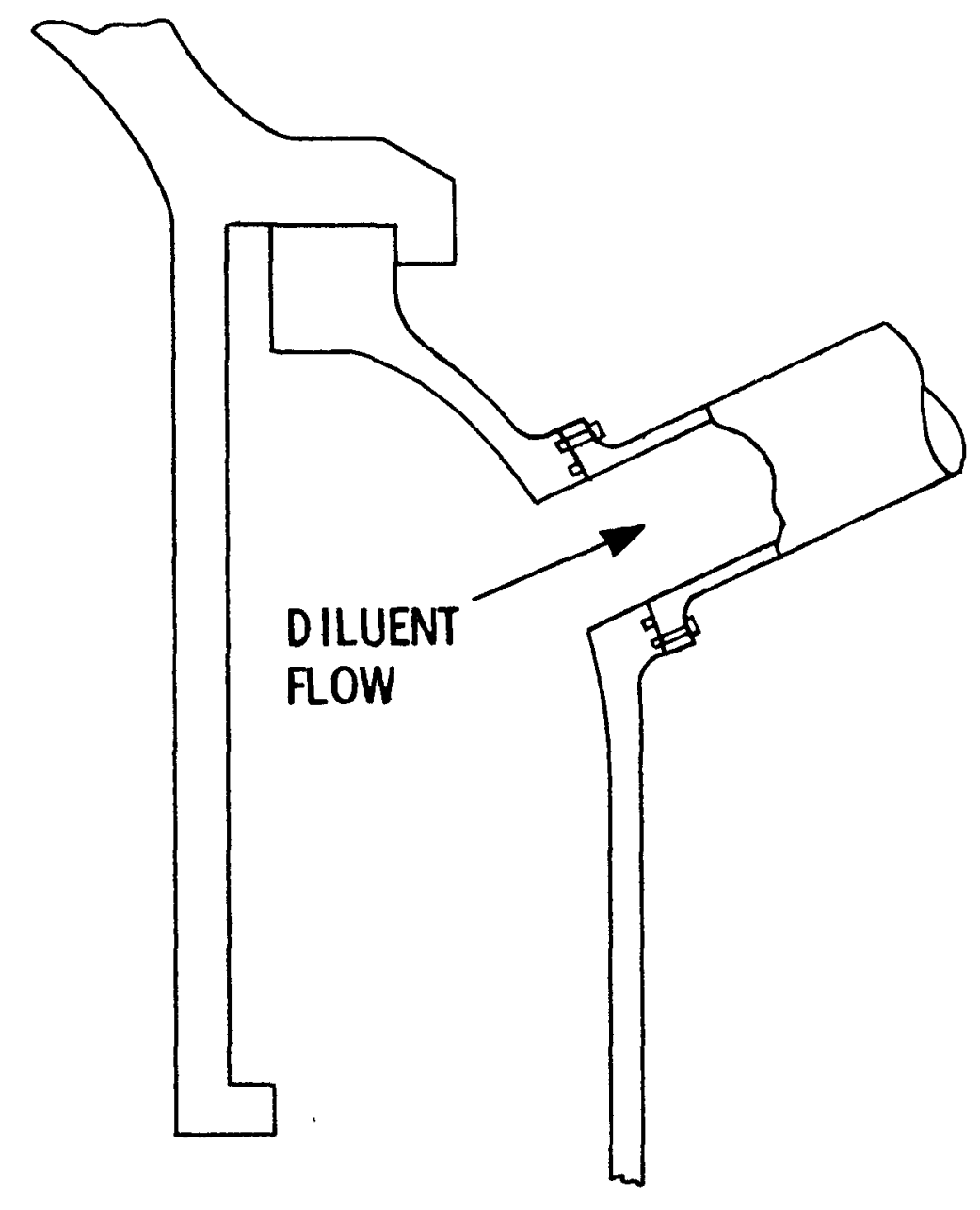

SINGLE PENETRATION 







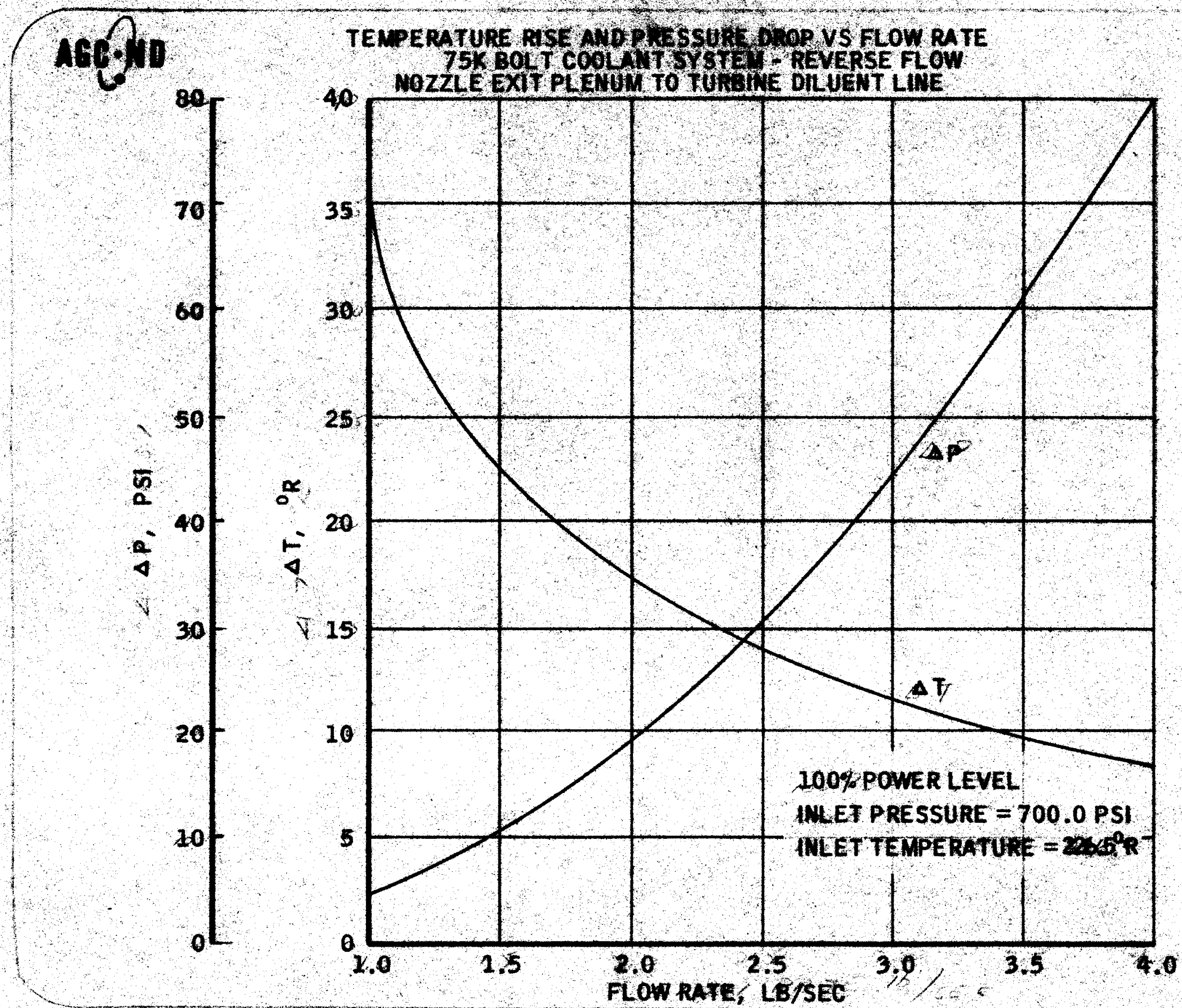




\section{BOLT COOLANT \& BILUENT FLOW TRADE STUDY \\ PERT EVENT S1165311-312 \\ PRELIMINARY FLUID CALCULATIONS SUMMARY}

OPERATING CONDITIONS:

1. 75K ENGINE FLOWING FROM NOZZLE OUTUET TO DILUENT INLET

2. NOZZLE OUTLET PRESSURE 680 PSIA

3. NOZZLE OUTLET TEMPERATURE $213^{\circ} \mathrm{R}$

4. BOLT COOLANT FLOW RATE 2.8 LB/SEC

\section{PRESSURE DROP SUMMARY:}

PASSAGE

DRILLED PASSAGES IN BOLT

NOZZLE FLANGE PASSAGES

OUTLET MANIFOLD

DILUENT LINE

DILUENT LINE VENTURI

BLEED PORT AND JACKETED ELBOW

(ESTIMATED FROM NERVA TECH EXPERIENCE)

TOTAL PRESSURE DROP

PRESSURE DROP AVAILABLE (NOZ OUTLET TO $P_{c}$ )

$\triangle P$ AVAILABLE FOR ORIFICING OR DILUENT CONTROL VALVE

\section{PRESSURE DROP (PSI)}

23.35

16.24

1.98

9.40

6.50

100.00

157.47

250.00

92.53 


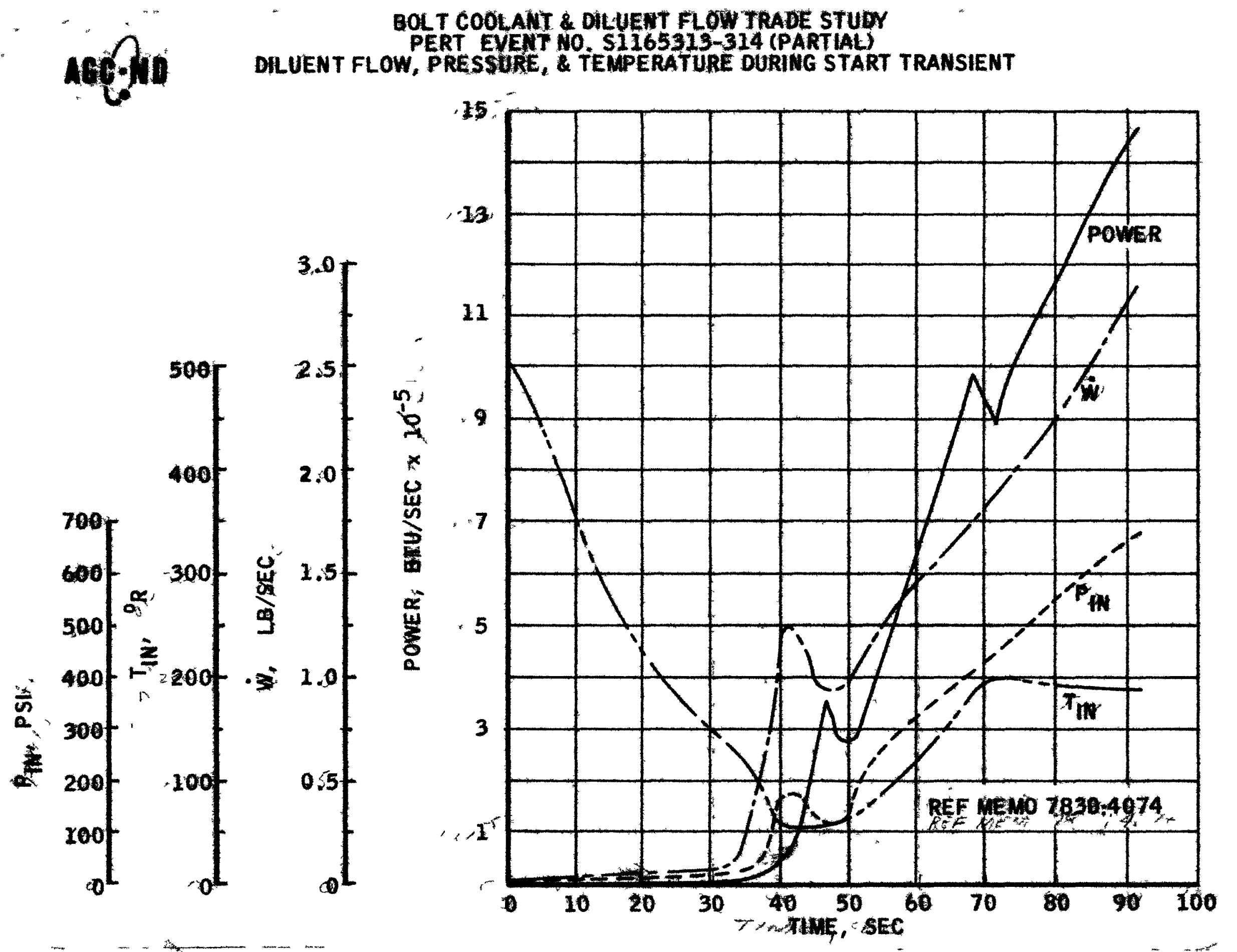

$269-254$ 


\section{BOLT COOLANT \& DILUENT FLOW TRADE STUDY}

PERT EVENT S 1165303 - C \$ 1165304

\section{ITEM}

1. DETERMINE DCV FLOW RESISTANCE COEFFICIENT AS A FUNCTION OF' SHAFT POSITION

2. DETERMINE LINE ROUTING, SIZES \& WEIGHTS NECESSARY TO STUDY ALL OPTIONS

3. DETERMINE MASS OF DCV
STATUS

1. COMPLETE - SEE VUGRAPH

2. NO WORK ACCOMPLISHED - COMPLETION DATE WILL SLIP 2 WEEKS

3. COMPLETE FOR 2 INCH LINE. WAITING FOR LINE SIZE CONFIRMATION PRIOR TO TRANSMITTI NG DATA TO SYSTEMS ENGINEERING 


\section{A6fing}

BOLT COOLANT \& DLUENT FLOW TRADE STUDY

DCV FL OW RESISTIMCE

PERT EVENT S1165303-304



$K=.447 \hat{A}^{2} S \Delta P N^{2}$

$A=$ IMLET AREA, $\mathrm{w}^{2}$

$S=$ SPECIFIC WEIGHT, LB/FT ${ }^{3}$

$P=$ PRESSURE, PSI

$\dot{w}=$ FLOW RATE, LB/SEC 


\section{BOLT COOLANT \& DILEENT FLOW TRADE STUDY PERT EVENT S1165323 - C1165324 ANALYZE EFFECTS OF RADIATION ON DCV}

$\underline{\text { ITEM }}$

1. DETERMINE

a. HEAT GENERATION RATES

b. POTENTIAL MATERIAL DAMAGE

2. EVALUATE GROSS HEATING EFFECTS ON DCV

3. PRELIMINARY EXTIMATE OF VALVE LOCATION FEASIBILITY
STATUS

NO WORK ACCOMPLISHED

START 2 WEEKS LATE

NO EFFECT ON COMPLETION DATE OF 24 APRIL 1969 
BOLT COOLANT \& DILUENT FLOW TRADE STUDY PERT EVENTS S1165319 -- C1165322

$\underline{\text { ITEM }}$

1. COLLECT \& ORGANIZE ALL DATA GENERATED DURING ANALYTICAL PHASE OF STUDY

2. OBTAIN DECISION ON OPTIMUM SYSTEM

3. WRITE TRADE STUDY REPORT

4. PUBLISH REPORT
STATUS

NO WORK ACCOMPLISHED SCHEDULED START DATE IS

3 JUNE 1969 


\section{BOLT COOLANT \& DILUENT FLOW TRADE STUDY PERT EVENT S1165317 - C1165318}

\section{STRESS ANALYSIS}

ITEM

1. RECEIVE LAYOUT FROM DESIGN GROUP

2. DEVELOP COMPUTER MODEL FOR NOZZLE/ PV JOINT AREA

3. PARAMETRIC STUDIES TO DETERAINE A COMPATIBLE DESIGN FOR MECHANICAL LOADING

4. DETERMINE LOADING CONDITIONS

(MECHANICAL \& THERMAL)

5. CONDUCT DETAILED ANALYSIS ON FLANGE AREA WITH REVERSE BOLT COOLANT CONFIGURATION

\section{STATUS}

1. LAYOUT IN WORK

2. - 5. NO WORK STARTED SCHEDULED START DATE 11 MARCH 1969 ESTIMATED COMPLETION DATE 3 JUNE 1969 


\section{BOLT COOLANT \& DILUENT FLOW TRADE STUDY}

\section{PERT EVENT S1165309-C1165310}

ITEM

1. RECEIVE INPUT FROM ANALYTICAL EFFORT

2. SEPARATE DESIGN ALTERNATES \& ORGANIZE DATA FOR RELIABILITY ANELYSIS

3. EVALUATE FAILURE MODE POTENTIAL OF EACH ALTERNATIVE SYSTEM

4. QUANTIFY RELATIVE RELIABILITY OF EACH SYSTEM

5. COMPARE \& RATE RELATIVE RELIABILITY

6. MAKE RELIABILITY RECOMMENDATION
STATUS

NO WORK ACCOMPLISHED. ACTIVITY SCHEDULED TO START 24 APRIL 1969 
BOLT COOLANT \& DILUENT FLOW TRADE STUDY

PERT EVENT S1165305-306

VARIABLE EXTRACTION POINTS FOR TURBINE DILUENT FLOW

ITEM

1. GENERATE STEADY STATE \& TRANSIENT PERFORMANCE PREDICTIONS FOR ENGINE SYSTEMS WITH DILUENT EXTRACTION POINTS AT THE FOLLOWING LOCATIONS:

a. PUMP DISCHARGE LINE

b. REFLECTOR INLET (BASE CASE CONFIGURATION)

C: REFLECTOR OUTLET
STATUS

FULL POWER COMPUTER RUNS COMPLETE, BUT NOT EVALUATED TRANSIENT RUNS NOT STARTED ESTIMATED COMPLETION DATE 11 MARCH 1969 
POTENTIAL ADVANTAGES

1. LOWER NOZZLE PRESSURE DROP

2. MAY LEAVE HIGHER NUMERICAL RELIABILITY DEPENDS ON MODE OF FAILURE

3. SIMPLER JACKET DESIGN IF EXTERNAL COOLING IS NOT REQUIRED

4. BETTER DILUENT - HOT GAS MKIMG CAPABILITY

5. ELIMINATES PRESENT STRUCTURAL \& DESIGN PROBLEMS ASSOCIATED WITH PRESENT SINGLE BLEED PORT
POTENTI AL PROBLEM AREAS AND DISADVANTAGES

1. NEW, UNTRIED CONCEPT

2. INCOMPLETE MIXING COULD CAUSE CATASTROPHIC FAILURE OF JACKET

3. CONTROL OF DILUENT MAY BE MORE DIFFICULT AND THERE IS NO PROVISION FOR A DILUENT CONTROL VALVE

4. $1660^{\circ} \mathrm{R}$ GAS AT EXTERNAL SURFACE OF JACKET PLUS RADIATION HE KTING COULD CAUSE JACKET STRUCTURAL \& HEATING PROBLEMS

5. COOLING OF ENTRANCE SECTION WOULD BE MORE DIFFICULT THAN PRESENT DESIGN

6. VERY DIFFICULT TO CORRECT IN CASE OF FAILURE COMPARED WITH BLEED PORT INSERT REPLACEMENT 


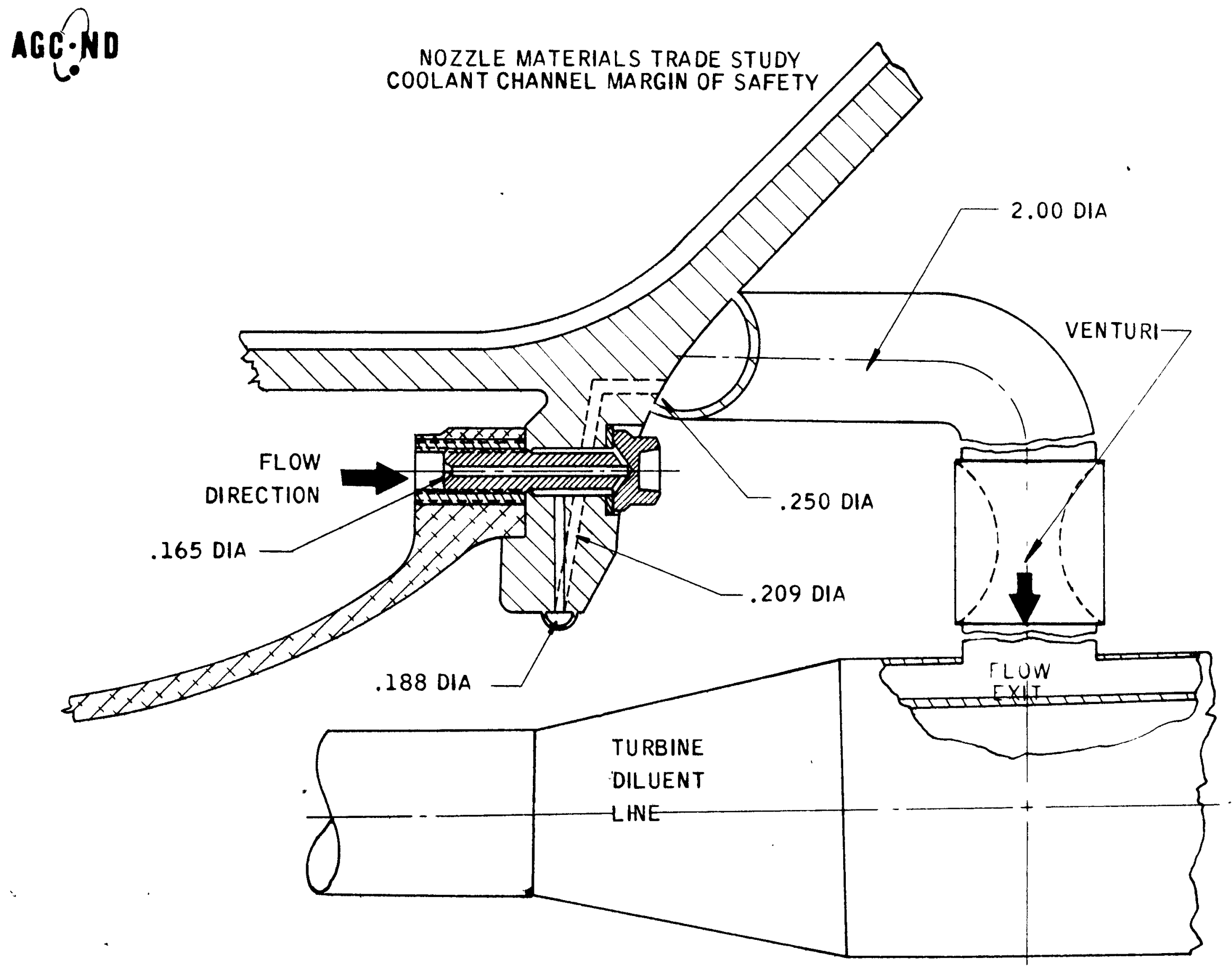


VIEW GRAPHS

Not USED

iN

Presentation 


\section{BOLT COOLANT \& DILUENT FLOW TRADE STUDY}

PERT EVENT S1165307-308

ITEMS

STATUS

1. DEMONSTRATE FEASIBILITY OF SERIES

COMPLETE:

BOLT COOLANT-DILUENT CONCEPT AT

MEMO 7830:M3803

ENGINE DESIGN OPERATING POINT

2. DEVELOP SIMPLIFIED MODEL OF SERIES

SEE VURRAPH

ESTIMATED COMPLETION

FLOW BOLT COOLANT/DILUENT CIRCUIT

DATE 24 MARCH 1969

FOR TRANSIENT ENGINE PROGRAM 31201

3. GENERATE START-TRANSIENT PREDICTIONS

ESTIMATED COMPLETION WITH SERIES FLOW MODEL

DATE 7 APRIL 1969

4. ADD DCV TO SYSTEMS MODEL \& GENERATE STEADY STATE \& TRANSIENT PREDICTIONS FOR ALTERNATE DILUENT FLOW SYSTEMS

SCHEDULED COMPLETION

DATE IN QUESTION 
BOLT COOLANT \& DILUENT FLOW TRADE STUDY PERT EVENT S1165311 - C C1165312

PRELIMINARY CALCULATIONS OF BOLT COOLANT CHANNEL GEOMETRY

ITEM

1. PREPARE LAYOUT OF BOLT COOLANT DILUENT SERIES FLOW SYSTEM

2. PERFORM FLUID CALCULATIONS TO SIZE PASSAGES \& ESTIMATE PRESSURE DROPS

3. INPUT DESIGN FOR SYSTEMS ANALYSIS

264.265
STATUS

COMPLETE - SEE VIEWGRAPH FOR SKETCH

COMPLETE - SEE VIEWGRAPH

COMPLETE - REF. MEMO 7750:M0294 


\section{BOLT COOLANT \& DILUENT FLOW TRADE STUDY \\ PERT EVENT S 1165313 - C1165314}

STEADY STATE PERFORMANCE CHARACTERISTICS - REVERSE B.C. SYSTEM

ITEM

$P_{I N}$, B.C. CIRCUIT(PSIA)

TIN, B.C. CIRCUIT $\left({ }^{\circ} R\right)$

W, DILUENT (LB/SEC)

$\triangle T$, IN B.C. CIRCUIT ( $\left.{ }^{\circ} R\right)$

TIN, DILUENT LINE ( $\left.{ }^{\circ} \mathrm{R}\right)$

$\triangle P$, TOTAL CIRCUIT (PSI)

$\triangle P$, B.C. CIRCUIT \& DILUENT LINE (PSI)

$\triangle P$, BLE ED PORT INSERT (PSI)

CONTROL PRESSURE AVAILABLE (PSIA)

TURBINE FLOW (LB/SEC)

TPCV GATE POSITION (DEGREES)

TURBINE INLET TEMP $\left({ }^{\circ} R\right)$

$\begin{array}{ll}\text { CASE 1 } & \text { CASE 2 } \\ 680 & 680 \\ 213 & 213 \\ 2.72 & 2.72 \\ 12 & 27 \\ 225 & 240 \\ 230 & 230 \\ 63 & 65 \\ 54 & 56 \\ 113 & 109 \\ 3.97 & 3.95 \\ 42.3 & 42.0 \\ 1660 & 1660\end{array}$

CONCLUSION: 75K NERVA ENGINE FULL POWER OPERATION COULD BE ACHIEVED WITH THE SERIES FLOW BOLT COOLANT-DILUENT SYSTEM, WITH POSITIVE CONTROL MARGINS FOR THE TPCV \& DILUENTILINE ORIFICE OR DCV. 
BOLT COOLANT \& DILUENT FLOW TRADE STUDY

PERT EVENT S1165313 - C C1165314

ITEM

1. PERFORM STEADY STATE SYSTEMS ANALYSES OF SERIES FLOW BOLT COOLANT - - DILUENT CIRCUIT

2. DEFINE TRANSIENT FLUID CHARACTERISTICS INPUT TO BOLT COOLANT CIRCUIT FOR TRANSIENT THERMAL ANALYSIS

\section{STATUS}

COMPLETE -- SEE VIEWGRAPH

REF. MEMO 7830:M3803

COMPLETE -- SEE VIEWGRAPH

REF. MEMO 7830:4074 


\section{BOLT COOLANT \& DILUENT FLOW TRADE STUDY}

\section{PERT EVENT S1165315 - C1165316}

ITEM

1. DETERMANE FLUID PRESSURE \& TEMPERATURE CHANGE THROUGH NOZZLE FLANGE UNDER TRANSIENT \& STEADY STATE CONDITIONS

2. PERFORM UPGRADED DETAILED THERMAL ANALYSIS OF NOZZLE - PV FLANGE AREA AT $30 \%, 60 \%, \& 200 \%$ POWER LEVELS TO OBTAIN METAL TEMPERATURE PROFILE
STATUS

STEADY STATE COMPLETE, SEE VUGRAPH TRANSIENT ANALYSIS IN PROCESS - SCHEDULED COMPLETION DATE 7 MARCH 1968

NO WORK STARTED - THE START OF THIS EFFORT IS BEING DELAYED PENDING RECEIPT OF NUCLEAR HEATING RATES. ESTIMATED START DATE IS 27 FEB 1969 
STUDY EFFECTS ON PRESSURE VESSEL STRUCTURE OF

LOCATING DILUENT PORT IN PRESSURE VESSEL

PERT EVENT S1165325-326

\begin{tabular}{|l|ll|}
\hline EFFORT & \\
\hline $\begin{array}{l}\text { 1. LAYOUT - DESIGN VARIATION } \\
\text { SIMGLE PENETRATI ON } \\
\text { MULTIPLE PENETRATIONS WITH } \\
\text { COMMON MANIFOLD }\end{array}$ & $1 . \quad$ IN PROCESS \\
$\begin{array}{l}\text { 2. AMALYSIS } \\
\text { EFFECT ON P.V. SHELL } \\
\text { STRESSES } \\
\text { EFFECT ON P.V. SHELL } \\
\text { TEMPERATURE }\end{array}$ & 2. $\quad$ FUTURE \\
3. FABRICATION & & \\
4. RELIABILITY & 3. FUTURE \\
5. COST & 4. FUTURE \\
\hline
\end{tabular}

$a-m \quad \ldots$

$2-25-6 y$

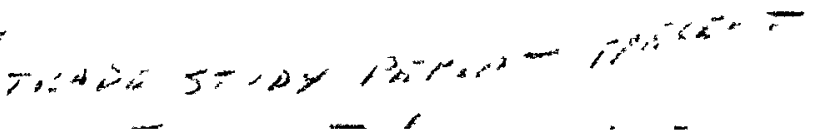


TEMPERATURE RISE ATD PRESSURE DROP VS FLOW RATE 75K BOL T COOLANT SYSTEM - FORWARD FLOW

BOLT COOLANT MAMIFOLD TO NOZZLE PLENUM

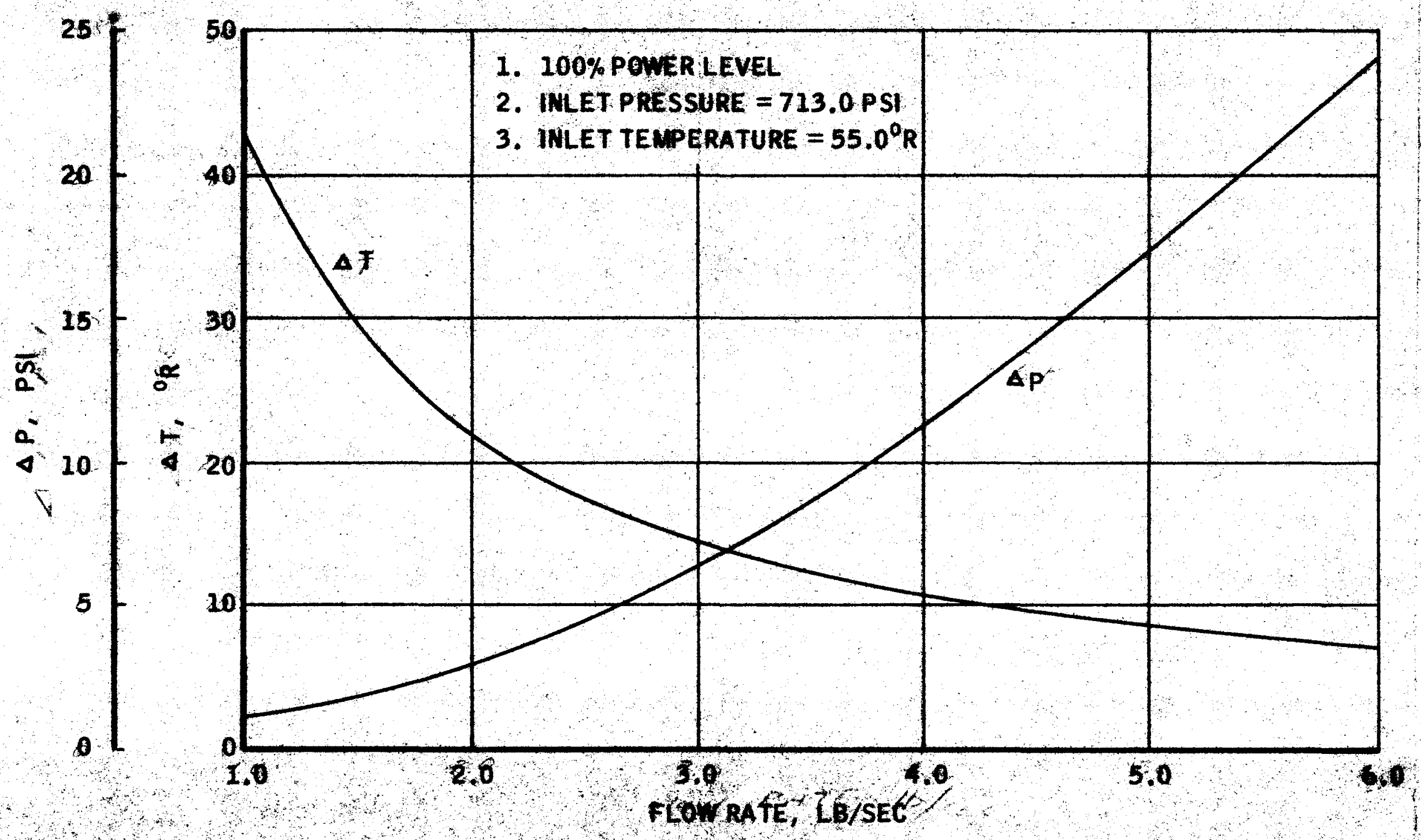


LIST OF TRADE STUDIES

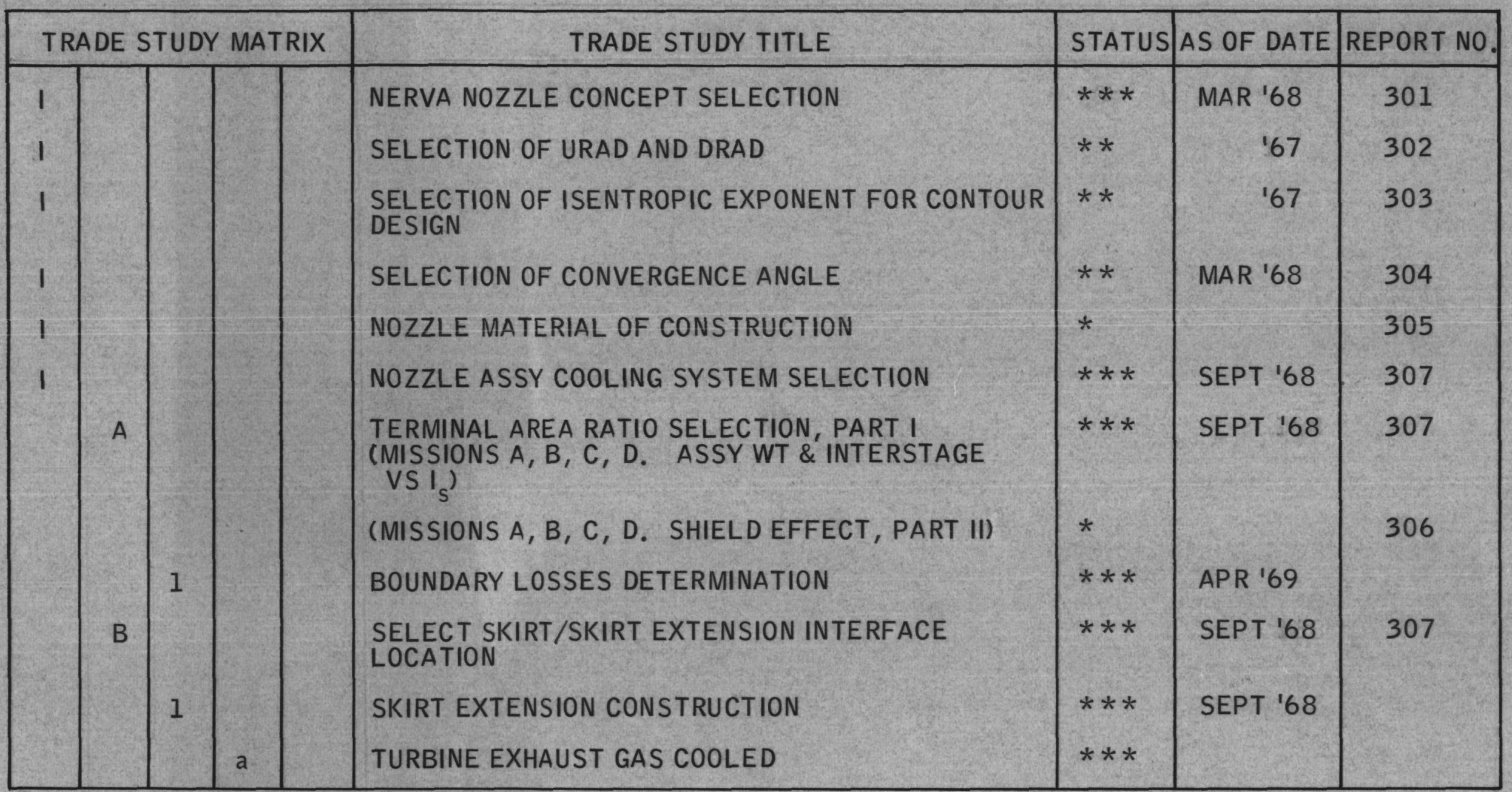

* IN PROCESS

** COMPLETED, EXCLUSIVE OF REPORT

*** PRELIMINARY REPORT WRITTEN

**** FORMAL REPORT WRITTEN

CONTINUED 


\section{AGC. AnO}

LIST OF TRADE STUDIES

\begin{tabular}{|c|c|c|c|c|c|c|c|}
\hline \multicolumn{4}{|c|}{ TRADE STUDY MATRIX } & TRADE STUDY TITLE & STATUS & AS OF DATE & REPORT NO. \\
\hline 1 & ; & $\begin{array}{l}\text { b } \\
c\end{array}$ & $\begin{array}{l}\text { (1) } \\
\text { (2) }\end{array}$ & $\begin{array}{l}\text { LIMITATION ON SKIRT EXTENSION COOLING } \\
\text { FEASIBILITY } \\
\text { EFFECT ON REFLECTOR INLET TEMPERATURE } \\
\text { RADIATIVELY COOLED } \\
\text { GRAPHITE } \\
\text { NOZZLE ASSY COOLING SYSTEM SELECTION } \\
\text { (CONTINUED) } \\
\text { NOZZLE/SKIRT INTERFACE LOCATION } \\
\text { NOZZLE/SKIRT PARALLEL VS SERIES FLOW } \\
\text { NOZZLE/SKIRT PARALLEL FLOW } \triangle P \\
\text { EFFECT OF COOLANT INLET LOCATION } \\
\text { NOZZLE/SKIRT SERIES FLOW } \triangle P \\
\text { SINGLE VS DOUBLE PASS FLOW } \\
\text { EFFECT OF COOLANT INLET LOCATION } \triangle P \text {, } \\
\text { WEIGHT, EQUIVALENT IS } \\
\text { R-SKIRT OPERATIONAL CONSIDERATIONS } \\
\text { SKIRT/SKIRT EXTENSION INTERCHANGEABILITY } \\
\text { EFFECT ON PERFORMANCE }\end{array}$ & $\begin{array}{l}* * * \\
\star * * \\
* * * \\
* * * \\
* * * \\
* * * \\
* *\end{array}$ & $\begin{array}{l}\text { SEPT '68 } \\
\text { SEPT ' } 68 \\
\text { SEPT '68 } \\
\text { MAR ' } 69\end{array}$ & $\begin{array}{l}309 \\
308\end{array}$ \\
\hline $\begin{array}{l}* \\
* * \\
* * * \\
* * * *\end{array}$ & $\begin{array}{l}\text { IN PI } \\
\text { COMI } \\
\text { PREL } \\
\text { FORI }\end{array}$ & MIN & POR & $\begin{array}{l}\text { CLUSIVE OF REPORT } \\
\text { PORT WRITTEN } \\
\text { WRITTEN }\end{array}$ & & & \\
\hline
\end{tabular}




\section{AGe.P.ND}

TRADE STUDIES IN PROGRESS

I. SELECTION OF TURBINE GAS DILUENT SOURCE

A. DOME (REFLECTOR OUTLET) WITH OR WITHOUT DILUENT FLOW CONTROL VALVE

B. REFLECTOR INLET ORIFICE FLOW CONTROL

C. BOLT COOLANT REVERSE FLOW - ORIFICE FLOW CONTROL

II. NOZZLE SKIRT EXTENSION SELECTION

A. RADIATIVELY COOLED

1. METALLIC

2. GRAPHITE

B. CONVECTIVELY COOLED

1. COOLED WITH TURBINE EXHAUST

2. CRES 347 CRYOGENICALLY COOLED

3. AL CRYOGENICALLY COOLED 
III. RE-EVALUATION OF SUITABILITY OF CRES 347 FOR NOZZLE CONSTRUCTION

IV. ALTERNATE MEANS OF TURBINE DRIVE FLUID CONSTRUCTION
A. PERIPHERAL FLOW WITH AND WITHOUT DILUENT
B. FROM SUBSONIC PORTION OF THE NOZZLE VIA MULTI INTERCOOLANT CHANNEL PASSAGES
C. FROM SUPERSONIC PORTION OF THE NOZZLE VIA MULTI INTERCOOLANT CHANNEL PASSAGES 


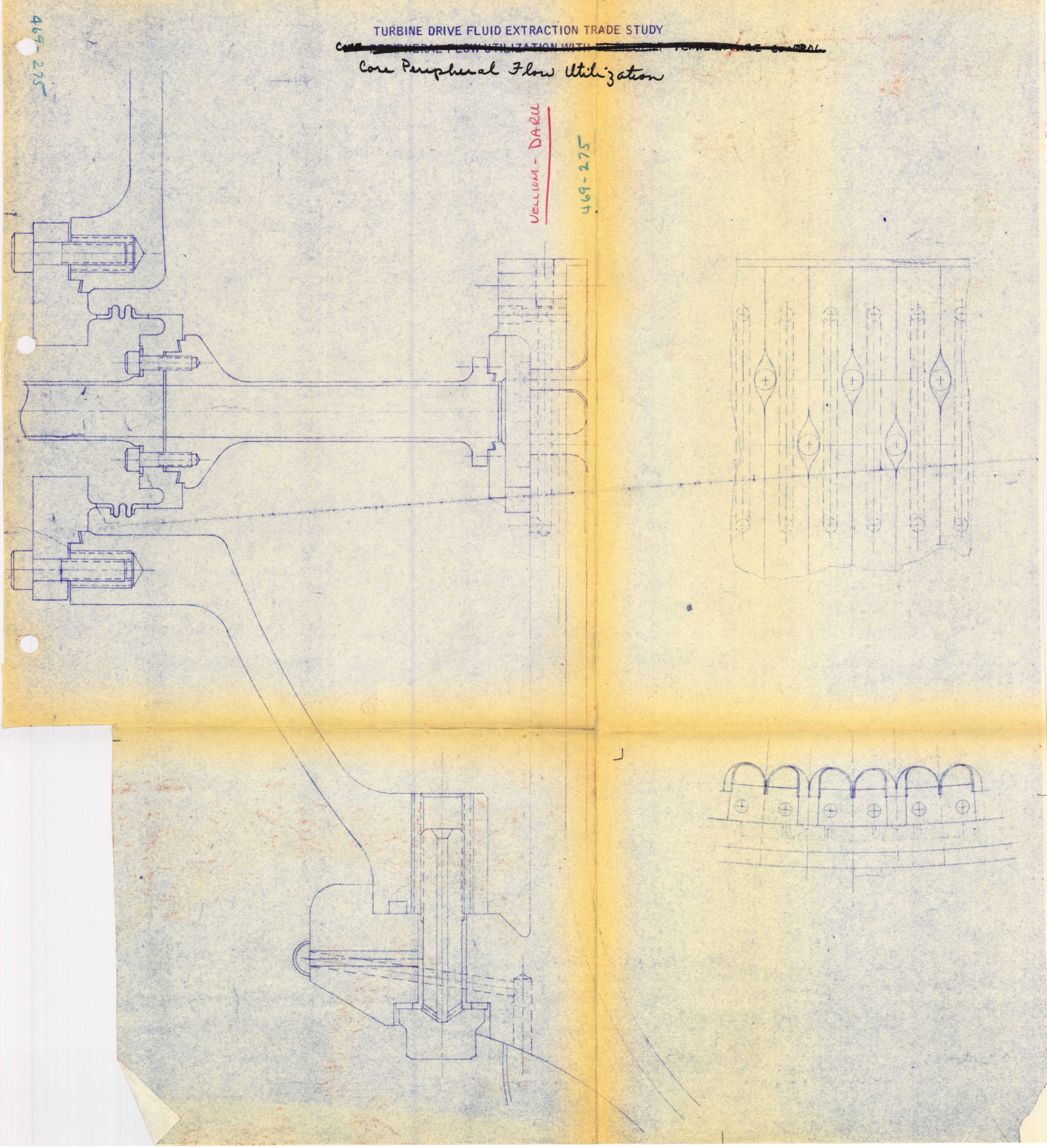


ADVANTAGES

1. NO DILUENT SYSTEM REQUIRED

2. LOWER NOZZLE PRESSURE DROP

3. NO DILUENT HOT GAS MIXING PROBLEMS

4. LESS HEAT FLUX AT GAS EXTRACTION HOLES

5. ALL NOZZLE COOLANT CHANNELS ARE AFFECTED TO THE SAME DEGREE

6. REDUCED CHANCES OF COMPROMISING THE MISSION BY FAILURE WITHIN ANY SINGLE PENETRATION

7. SYSTEM SUITABLE FOR INCORPORATING TWO TOTALLY INDEPENDENT TIL'S

\section{DISADVANTAGES}

1. DIFFICULTIES IN PREDICTING THE AXIAL LOCATION AT WHICH $1660^{\circ} \mathrm{R}$ GAS COULD BE EXTRACTED

2. WANL WOULD BE REQUIRED TO MAKE DESIGN CHANGES TO MODIFY REFLECTOR SUPPORT METHOD \& PROVIDE MORE LATERAL SUPPORT COOLANT FLOW

3. PRESSURE VESSEL WOULD REQUIRE REDESIGN

4. LESS TURBINE INLET PRESSURE THAN WITH PRESENT DESIGN

5. ADDITIONAL BOLTS, SEALS \& BELLOWS WOULD PROBABLY DEGRADE RELIABILITY

6. CONCEPT COULD NOT BE PROVEN BY CHEMICAL TESTING

7. FABRICATION WOULD BE MORE DIFFICULT AND COSTLY THAN PRESENT DESIGN

8. MULTI PENETRATIONS THROUGH THE PRESSURE VESSEL WALL 
TURBINE DRIVE FLU XTRACTION TRADE STUDY

CORE PERIPHERAL FLOW UTILIZATION WITH TEMPERATURE CONTROL
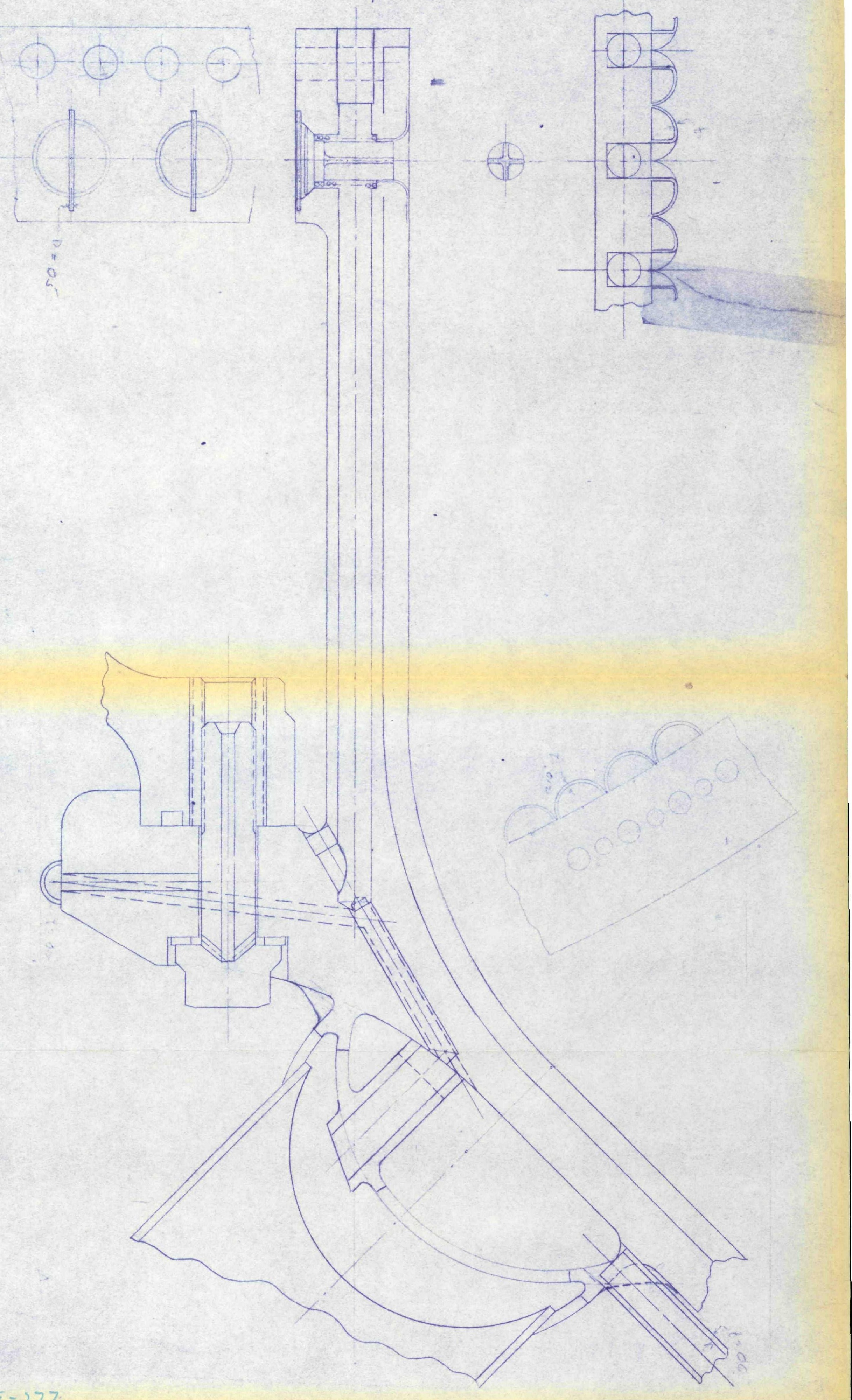

$469-277$ 







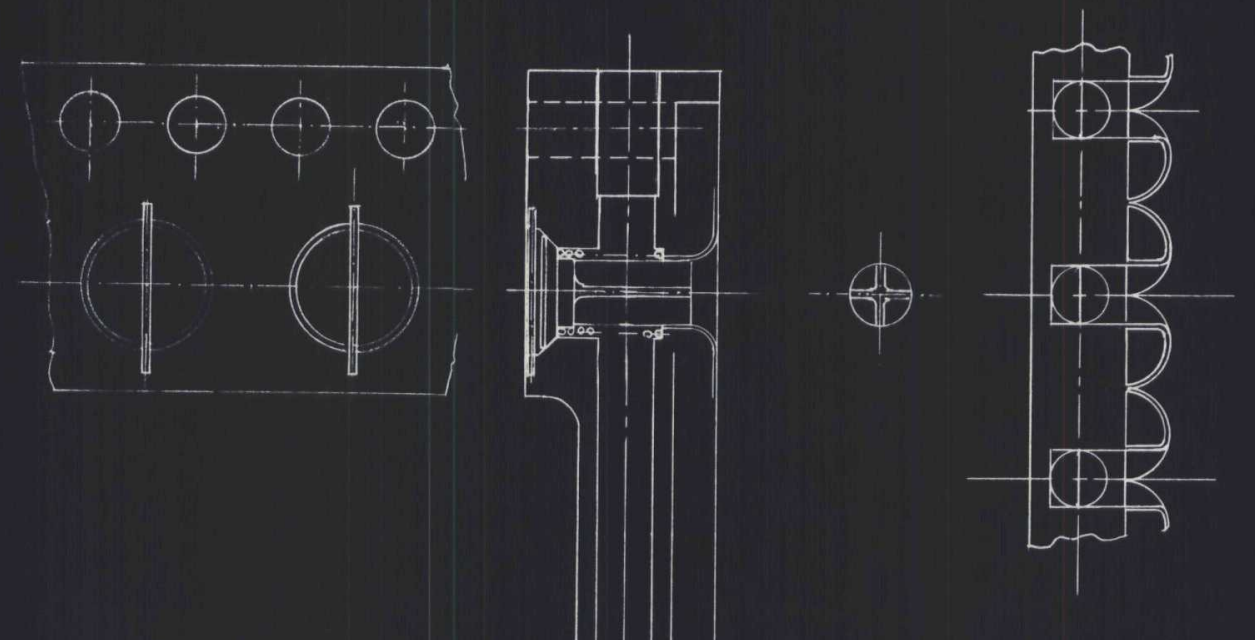

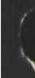




\section{ADVANTAGES}

1. SYSTEM WOULD BE PROTECTED FROM EXCESS TEMPERATURE TURBINE DRIVE GAS

2. ENERGY TO TURBINE COULD BE CONTROLLED EXCLUSIVELY BY TPCV

3. LOWER NOZZLE PRESSURE DROP

4. LESS HEAT FLUX AT GAS EXTRACTION HOLES

5. ALL NOZZLE COOLANT CHANNELS ARE AFFECTED TO THE SAME DEGREE

6. REDUCED CHANCES OF COMPROMISING THE MISSION BY FAILURE WITHIN ANY SINGLE PENETRATION

7. SYSTEM SUITABLE FOR INCORPORATING TWO TOTALLY INDEPENDENT TIL'S

\section{DISADVANTAGES}

1. ACCURACY AND RELIABILITY OF TEMPERATURE COMPENSATING VALVE WOULD BE QUESTIONABLE

2. REFLECTOR SUPPORT SYSTEM WOULD REQUIRE REDESIGN TO PROVIDE SUFFICIENT LATERAL SUPPORT COOLING FOR TURBINE DRIVE FLUID

3. MAINTAINING STRUCTURAL INTEGRITY AND RELIABILITY OF NOZZLE AS AFFECTED BY LARGE NUMBER OF DRILLED PASSAGES MORE DIFFICULT

4. LESS TURBINE INLET PRESSURE

5. CONCEPT COULD NOT BE PROVED BY CHEMICAL SIMULATION TESTING

6. FABRICATION WOULD BE MORE DIFFICULT AND COSTLY THAN PRESENT DESIGN 
TURBINE DRIVE FUID EXTRACTION TRADE STUDY

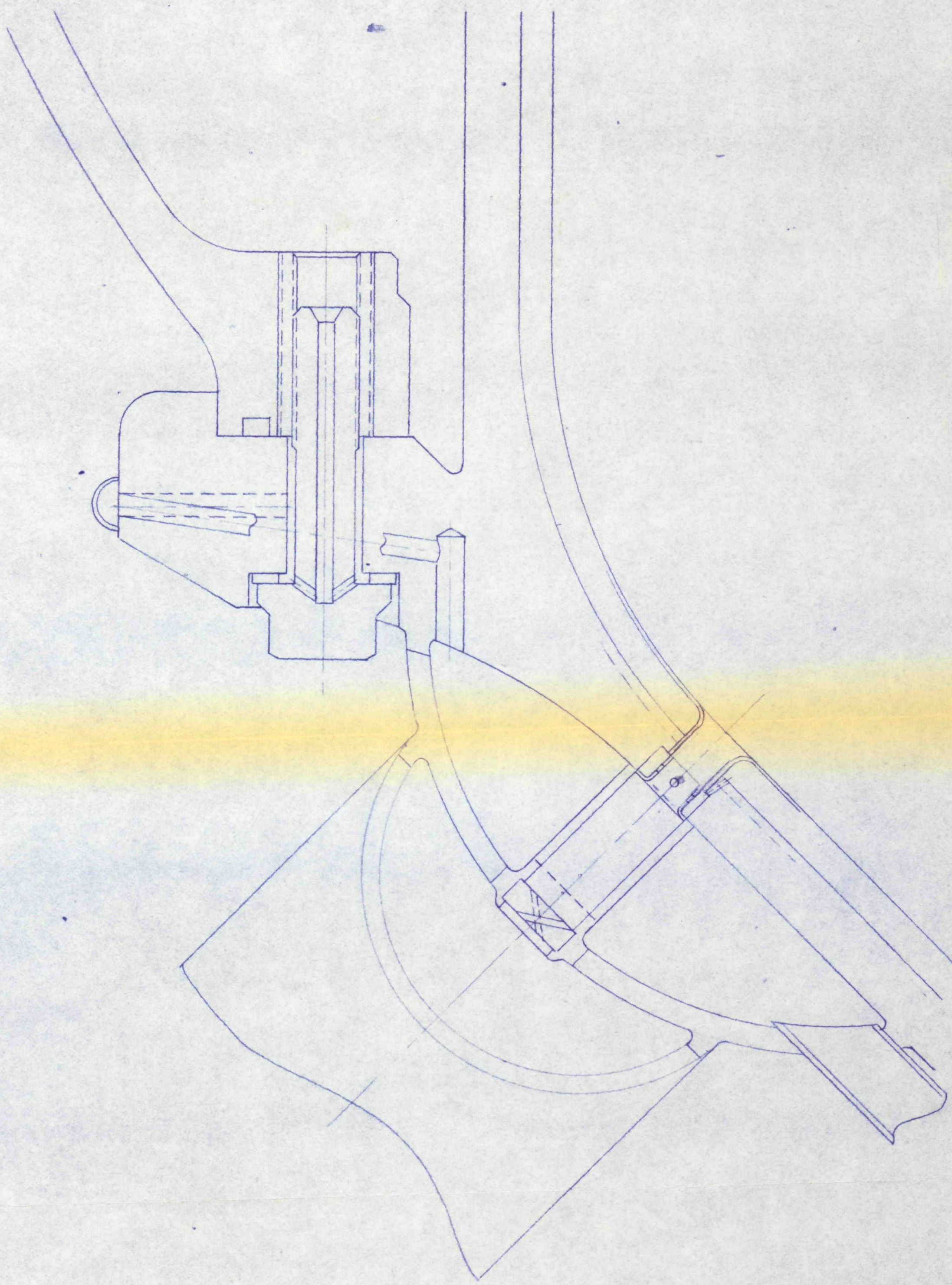




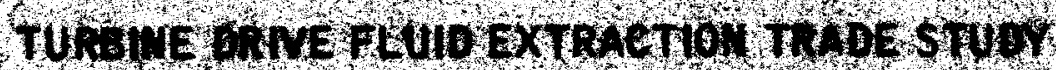

\section{COOLALT TAP-OFE FOR OLUEWT}

\section{ADVWTACES}

1, ELIMUNTES OLUENT SUPPLY SYSTEMS

2. LESS WOZZLE COOLANT PRESSURE DROP

3. ALL NOZZLE COOLANT CHANMELS ARE AFFECTED TO THE SAIE DECREE

4. REOUCEO CHANCES OF COMPROMISING THE MSSIOU BY FALURE WITHW ANY SINGLE PEUETRATION

5. SYSTEM SUITABLE FOR INCORPORATING TWO TOTALLY IMOEPENDENT TIL'S

\section{Gsavintacs}

1. NO METHOD OF COUTROLLUO DILUEMT FLOW

2. PROBABLY LESS TURBUE WLET PRESSURE

3. BURWOUT OF AWY OUE HOT CIS PASSACE COULD AFFECT JACKET STRUCT URAL WTEGRITY

4. SWALL OL UEUT FEED HOLES ARE SUSCEPTIBLE TO BEING PLUEGED WTH CONTAMWANTS

5. FABRICATION NORE DIFFICULT AND COSTLY

6. POTENTIAL LEAKACE AT SLEEVE TO TUEE BRAZE JOUNTS

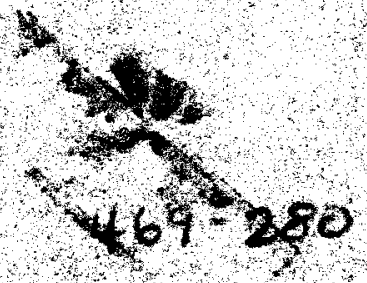




\section{ADVANTAGES}

1. BOLT COOLANT SYSTEM CAN BE USED AS A DILUENT SUPPLY SYSTEM

2. LESS NOZZLE COOLANT PRESSURE DROP

3. ALL NOZZLE COOLANT CHANNELS ARE AFFECTED TO THE SAME DEGREE

4. REDUCED CHANCES OF COMPROMISING THE MISSION BY FAILURE WITHIN ANY SINGLE PENETRATION

5. SYSTEM SUITABLE FOR INCORPORATING TWO TOTALLY INDEPENDENT TIL'S

\section{DISADVANTAGES}

1. NO ADEQUATE METHOD OF DILUENT CONTROL

2. FAILURE OF ANY ONE HOT GAS PASSAGE COULD AFFECT JACKET STRUCTURAL INTEGRITY

3. SMALL DILUENT FEED HOLES ARE SUSCEPTIBLE TO BEING PLUGGED WITH CONTAMINANTS

4. PROBABLY LESS TURBINE INLET PRESSURE

5. BRAZING HOT GAS PASSAGE SLEEVES TO TUBES AND TUBES TO JACKET COULD CAUSE LEAKAGE 



\section{AGC.ND}

TURBINE DRIVE FLUID EXTRACTION TRADE STUDY

CONTROLLED DILUENT CONCEPT

\section{ADVANTAGES}

1. DILUENT CAN BE CONTROLLED

INDEPENDENT OF HOT GAS

2. LESS NOZZLE PRESSURE DROP

3. ALL NOZZLE COOLANT CHANNELS ARE AFFECTED TO THE SAME DEGREE

4. REDUCED CHANCES OF COMPROMISING THE MISSION BY FAILURE WITHIN ANY SINGLE PENETRATION

5. SYSTEM SUITABLE FOR INCORPORATING TWO TOTALY INDEPENDENT TIL'S

\section{DISADVANTAGES}

1. BURNOUT OF ANY ONE HOT GAS PASSAGE COULD AFFECT JACKET STRUCTURAL INTEGRITY

2. PROBABLY LESS TURBINE INLET PRESSURE

3. FABRICATION MORE DIFFICULT AND COSTLY 


\section{AGe.}

PROPERTIES TEST PLAN

AG CARB-101

\begin{tabular}{|l|l|c|}
\hline TYPE OF TEST & $\begin{array}{l}\text { TEST } \\
\text { TEMP }{ }^{\circ} \mathrm{F}\end{array}$ & $\begin{array}{c}\text { NO } \\
\text { TESTS }\end{array}$ \\
\hline 1. PRELIM TENSILE & R.T. & $* 80(80)$ \\
2. TENSILE & R.T. & $46(48)$ \\
& 2000 & $35(30)$ \\
& 3000 & $35(30)$ \\
3. HOOP & R.T. & $14(9)$ \\
& 2000 & $0(6)$ \\
4. BIAS TENSILE & R.T. & $10(10)$ \\
& 2000 & $0(10)$ \\
& 3000 & $10(0)$ \\
5. BLOCK TENSILE & R.T. & $30(30)$ \\
6. COMPRESSION & R.T. & $22(30)$ \\
& 3000 & $8(0)$ \\
7. INTERLAM SHEAR & R.T. & $32(30)$ \\
8. FLEXURAL PARALLEL & R.T. & $20(40)$ \\
TOPLIES & 2000 & $20(40)$ \\
9. THERMAL DIFFUSIVITY & 3000 & $20(0)$ \\
9. THERMAL CONDUCTIVITY & 1500 & $6(0)$ \\
& 1500 to & \\
& 3000 & \\
\hline 5IGNIP to & \\
\hline
\end{tabular}

\begin{tabular}{|c|c|c|}
\hline TYPE OF TEST & $\begin{array}{l}\text { TEST } \\
\text { TEMP. }{ }^{\circ} \mathrm{F}\end{array}$ & $\begin{array}{l}\text { NO. } \\
\text { TESTS }\end{array}$ \\
\hline 10. FATIGUE $10^{3}$ to $10^{6}$ CYCLES & $\begin{array}{l}\text { R.T. } \\
1500 \\
2000\end{array}$ & $\begin{array}{l}36(35) \\
34(0) \\
0(35)\end{array}$ \\
\hline 11. ELECT. RESISTIVITY & R.T. & $5(10)$ \\
\hline 12. THERMAL EXPANSION & $R \cdot T$, to & $6(0)$ \\
\hline & $3000^{\text {R. to }}$ & $6(6)$ \\
\hline 13. FRACTURE TOUGHNESS & R.T. & $48(48)$ \\
\hline 14. HARDNESS & R.T. & $30(30)$ \\
\hline 15. POROSITY & R.T. & $3(3)$ \\
\hline 16. NENSITY & R.T. & $3(3)$ \\
\hline $\begin{array}{l}\text { 17. DYNAMIC MODULUS \& } \\
\text { DAMPING FACTOR }\end{array}$ & 2000 & $6(6)$ \\
\hline 18. PERMEABILITY & R.T. & $3(0)$ \\
\hline
\end{tabular}

SIGNIFIES REJISED TEST PLAN

( ) JIGNIFIES PREUIOUSGY SHOWN TEST PLAN 


\section{CIRCUMFERENTIAL $0.2 \%$ OFFSET YIELD STRENGTH \\ FOR TYPE 347 STAINLESS STEEL TAKEN FROM}

NERVA I NOZZLE SN-027

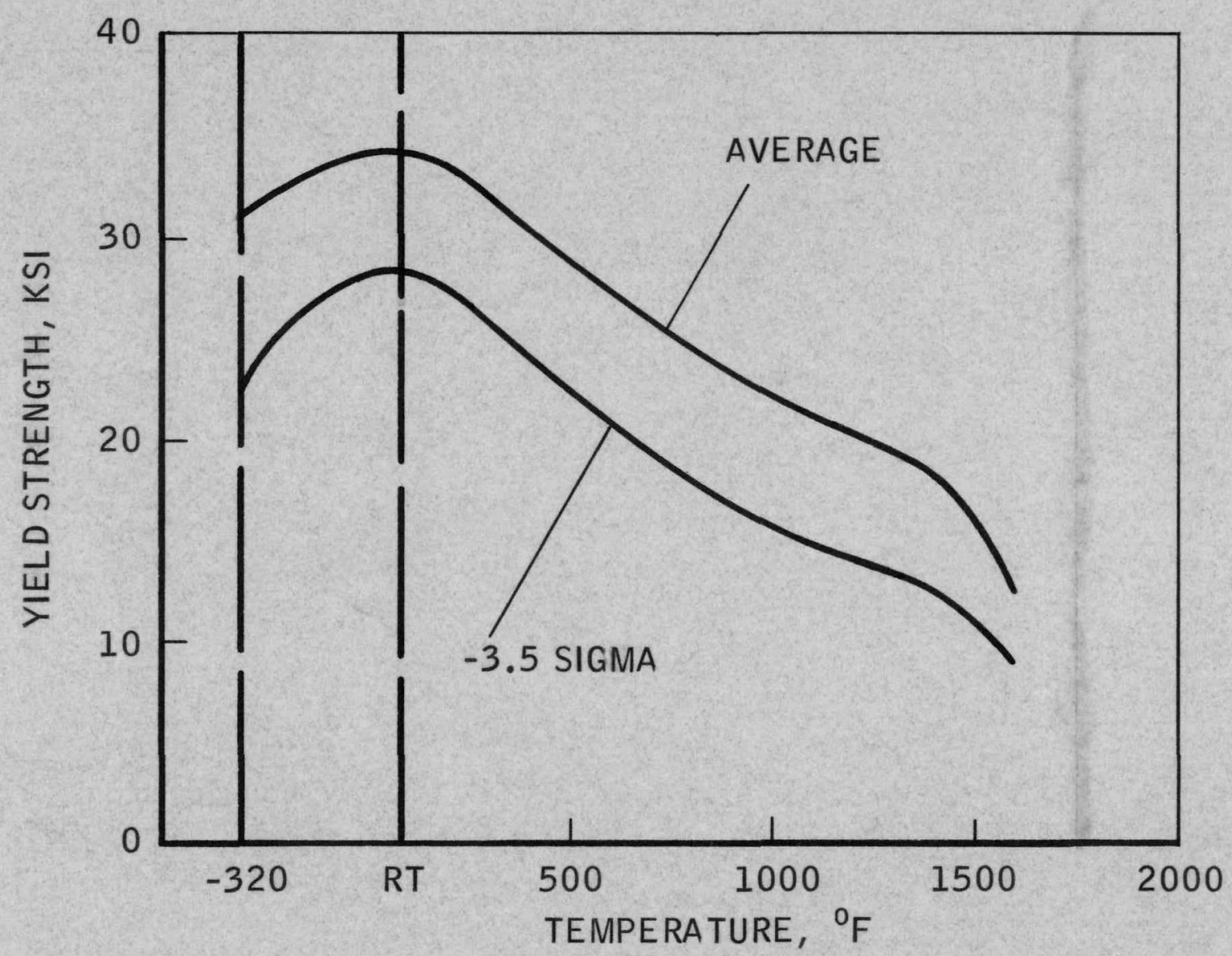




\section{JACKET RELIABILITY EVALUATION MAX STRESS POINT}

$$
\begin{aligned}
& \bar{s}=\frac{1.1 P R}{t \cos 6} \\
& \bar{s}=.85 \text { AVG TEST DATA }
\end{aligned}
$$

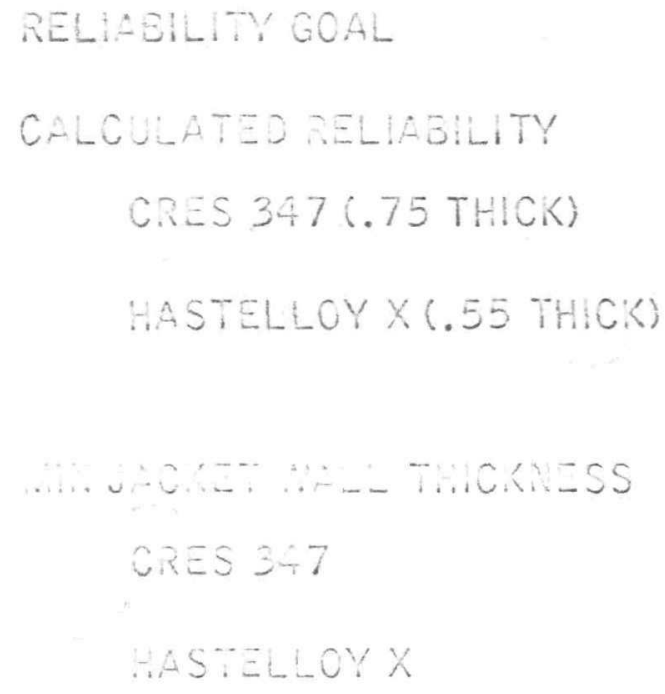


COOLANT TUBE RELIABILITY EVALUATION

NOZZLE AFT END - PRIMARY STRESS ONLY - $\underline{\underline{S}}=P R R$

$\bar{S}=.85$ AVG TEST DATA

RELABHLYGOAL $\quad 9_{5} 85$ (REF. MEMO 78400726)

CALCULATEO RELIABILITY (OPERATHNG CONOHIOASI

\begin{tabular}{|c|c|c|}
\hline CRES 3 TTUBE & RELIABILITY & OAROM OF SAREYY \\
\hline $0.012 \mathrm{kALL}$ & .9705 & .30 \\
\hline $0.02 \angle \mathrm{WALL}$ & $.911{ }^{70}$ & .60 \\
\hline \multicolumn{3}{|l|}{ HASTELLOY X } \\
\hline $0,072 \mathrm{WALL}$ & .9370 & 1.09 \\
\hline
\end{tabular}




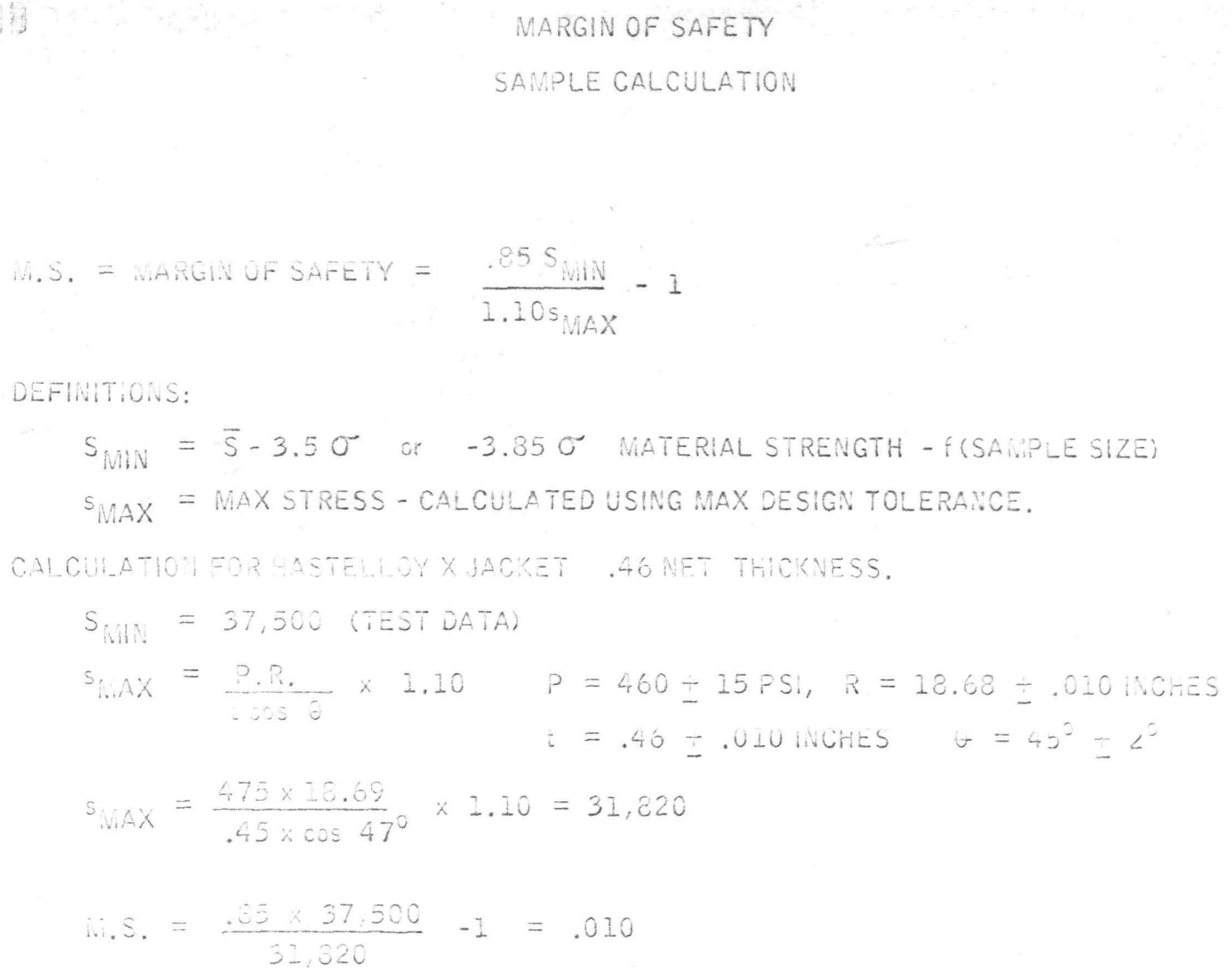


$\bullet$

AGG.NO

SAMPLE RELIABILITY CALCULATION

PROBLEM: DETERMINE RELIABILITY OF 0.51 THICK HASTELLOY X JACKET.

CONSTANTS: MEAN JACKET WALL TEMP $T=175^{\circ} \mathrm{F}-\mathrm{RP}$, TR: 0385

MEAN MATERIAL STRENGTH $\vec{S}_{1}=42 \mathrm{KSI}-$ TEST DATA

INTERNAL GAS PRESSURE $P=460$ PSI - COMPUTERTAB.

JACKET RADIUS $\quad R=18.68$ IN. - LAYOUT

JACKET WALL ANGLE $\quad \theta=45^{\circ}$ - LAYOUT

DEFINITIONS: $C=$ COEFFICIENT OF VARIATION OF RELIABILITY

$F=$ SAFETY FACTOR $=\bar{S} / \bar{S}$

$\sigma_{\mathrm{S}}=$ STANDARD DEVIATION OF STRENGTH

$\sigma_{S}=$ STANDARD DEVIATION OF STRESS

$t=$ JACKET WALL THICKNESS $-.5 \mathrm{IN}$ 
SAMPLE RELIABILITY CALCULATION CONTINUED

$$
\begin{aligned}
& \bar{S}=.85 \bar{S}_{1}=.85 \times 42,000=35,600 \mathrm{PSI} \\
& \bar{s}=\frac{P R 1.10}{t \cos \theta}=\frac{460 \times 18.68 \times 1.10}{.46 \cos 45^{\circ}}=29,065 \mathrm{PSI} \\
& \mathrm{F}=\frac{\bar{s}}{\bar{s}}=\frac{35,000}{29,065}=1.22 \\
& c=\sqrt{\sigma_{\mathbf{S}}^{2}+\sigma_{\mathrm{S}}^{2} / \bar{S}}=\quad \sigma_{\mathbf{S}}=1280 \text { CALCULATED VALVE } \\
& \mathrm{C}=1280^{2}+310^{2} \quad 35600 \quad \sigma_{\mathrm{S}}=310 \text { CALCULATED VALVE } \\
& C=0.037
\end{aligned}
$$

RELIABILITY ESTIMATED FROM DR. KECECLOGLU'S GRAPH* $=96^{2}$

CALCULATED RELIAG LITY USING NORMAL PROBABILITY FUNCTION.

$$
\begin{aligned}
& x=\bar{s}-\bar{s} \sqrt{\sigma_{s}^{2}+\sigma_{s}^{2}} \\
& x=5.10 \\
& \text { RELLIABILITY } 9_{6} 83
\end{aligned}
$$

* A UNIFIEd LOOK AT DESIGN SAFETy FACTORS. KECEClOglu PG 7 
VIEWGRAPHS NOT USED IN PRESENTATION-NO MASTER VELLUIMS " FILED IN MASTER FILE AT REAR OF SECTION IN FOLDER

1. BOLT COOLANT-DILUENT CONCEPT

2 COOLANT CHANNEL DILUENT CONCEPT

3 PERIPHERAL FLOW UTILIZATION CONCEPT WITH TEMPERATURE COMPENSATING VALVE 4 DILUENT CONTROL CONCEPT 
- 


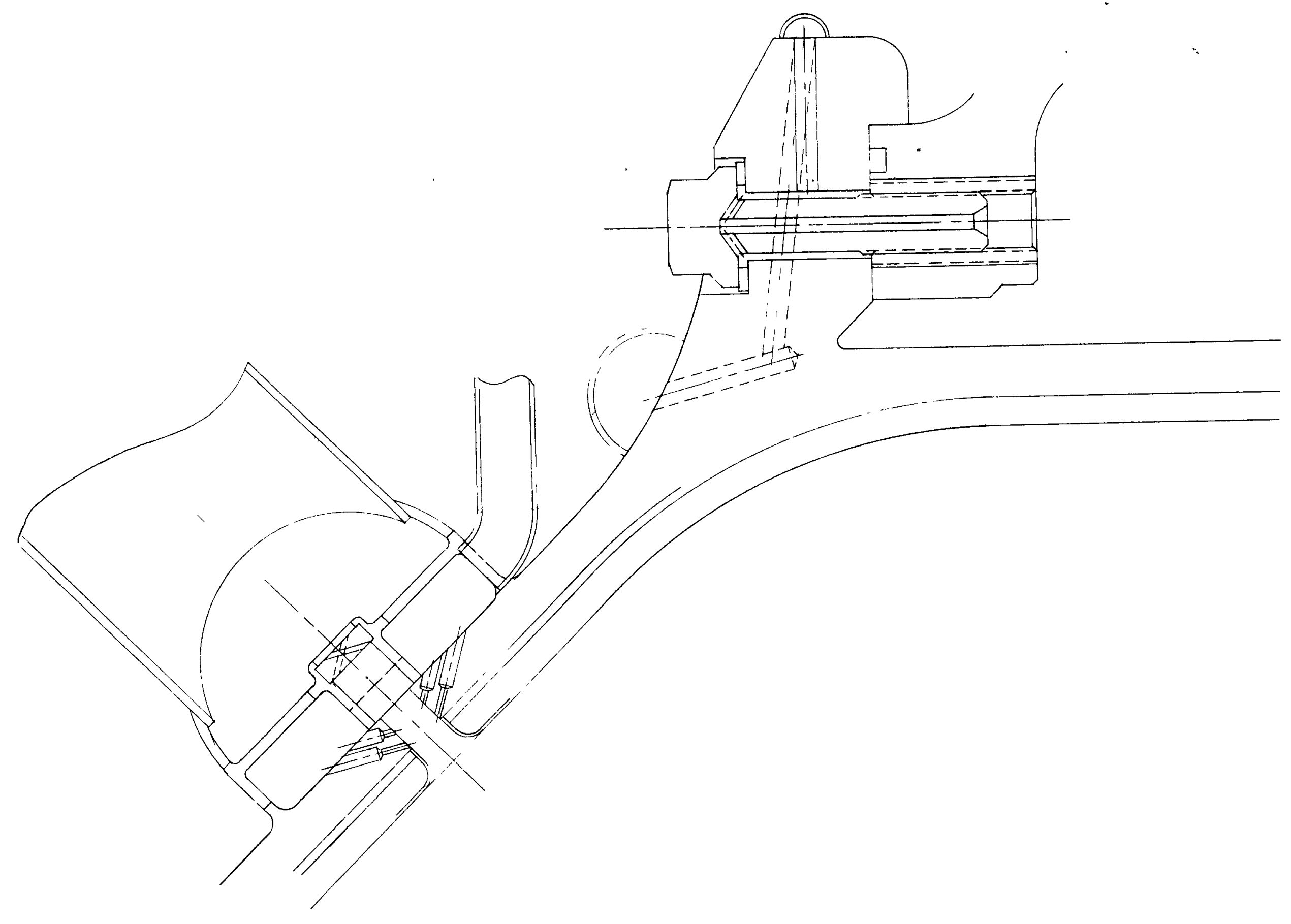




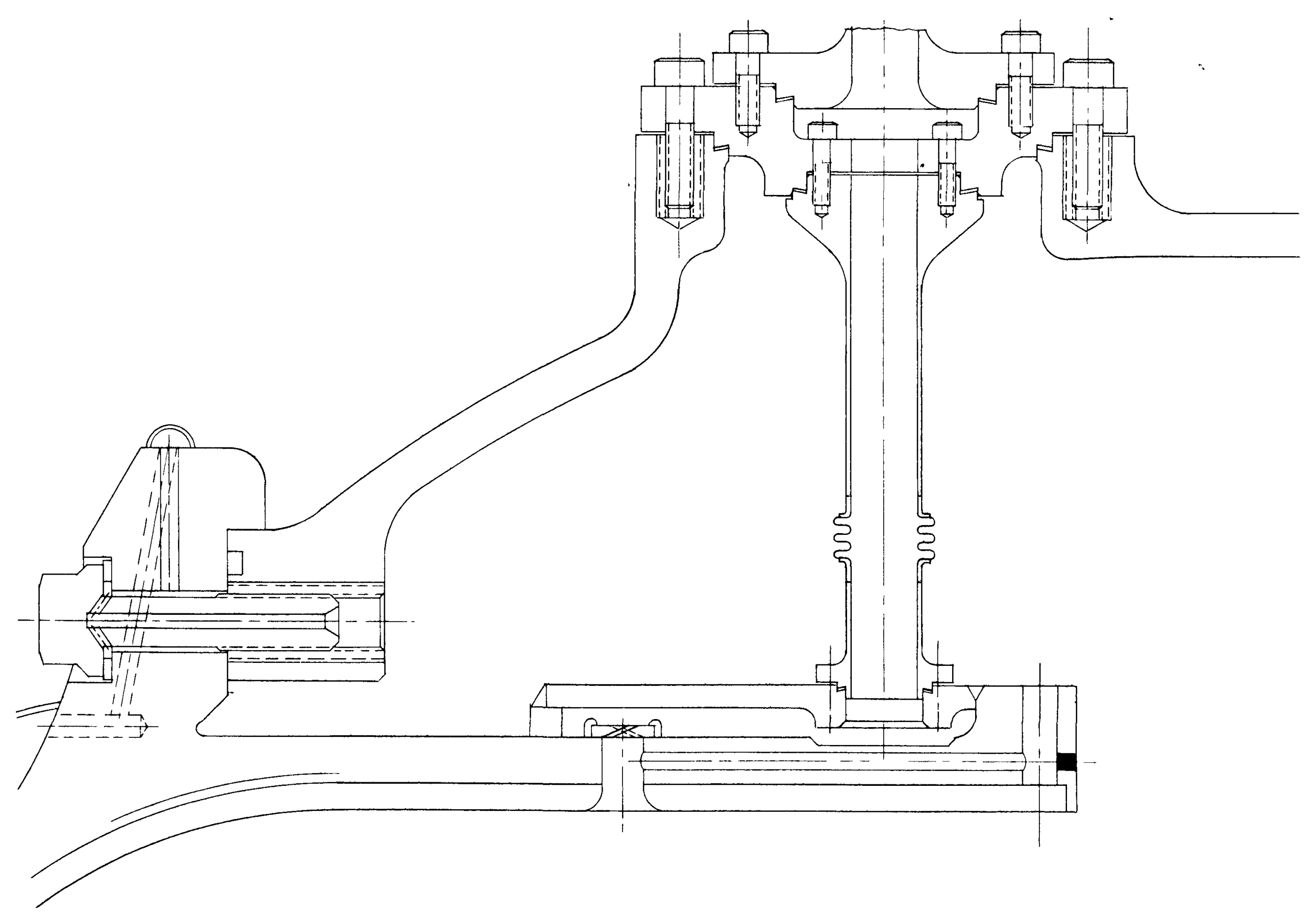




\section{NOZZLE}

GROSS INFORMATION FOR PDR IN DEC. 1969

KNOWN, NOW

1. 'U' TUBE TYPE CONVECTIVELY COOLED NOZZLE WITH CONSTRUCTION SIMILAR TO TECHNOLOGY AND PHOEBUS NOZZLES.

2. TURBINE DRIVE EXTRACTION SYSTEM WILL BE REQUIRED.

3. INTERFACE DIMENSIONS WITHIN \pm .25 INCHES.

4. NOMINAL (STEADY STATE ONLY) PROPELLANT CONDITIONS NECESSARY FOR NOZZLE SIZING.

5. NOZZLE COOLANT FLOW IN SERIES WITH SKIRT.

UNKNOWN, NOW

1. TYPE OF TURBINE DRIVE EXTRACTION SYSTEM.

2. NOZZLE MATERIAL (HASTELLOY ' $X$ ' OR CRES 347).

3. DETAIL FLUID CONDITIONS WITH TOLERANCES.

4. REACTOR INTERFACE LOADS. 


\section{AGC.ND}

GROSS INFORMATION FOR PDR IN DEC. 1969/CONT.

ASSUMPTIONS NECESSARY TO ARRIVE AT DEC PDR

1. NOZZLE MATERIAL - CRES 347.

2. ONE BLEED PORT INSERT.

3. DETAIL FLUID CONDITIONS WITH TOLERANCES AVAILABLE BY 8-15-69.

4. REACTOR INTERFACE LOADS AVAILABLE BY 7-1-69.

5. RELIABILITY WORK RESTRICTED TO A QUALITATIVE FAILURE MODE ANALYSIS.

6. NOZZLE DESIGN BASED ON STEADY STATE CONDITIONS AND CRITICAL TRANSIENT CONDITIONS ( IF REQUIRED) USING MAXIMUM TOLERANCES RATHER THAN STATISTICAL AVERAGES.

7. FORWARD BOLT COOLANT SYSTEM WITH EXTERNAL BOLT COOLANT LINES USED TO COOL FLANGE.

8. RAO CONTOUR, WITH $A_{e} / A_{t}=100$

9. NOZZLE SKIRT INTERFACE AT $A_{e} / A_{t}=5: 1$ 
KNOWN:

1. INTERFACE CONFIGURATION TO NOZZLE

2. HOT GAS CONDITIONS

3. FOUR PORT INSERT MODELS REQUIRED

UNKNOWN:

1. WHICH FOUR MODELS WILL BE SELECTED

2. MIXED GAS REQUIREMENTS PLUS TOLERANCE

3. DILUENT FLUID CONDITIONS PLUS TOLERANCE

4. ONE OR TWO PORT INSERTS

5. TURBINE INLET LINE INTERFACE CONFIGURATION

ASSUMPTIONS: $\quad$ 1. THE FOUR MODELS SELECTED ARE

A. CRES 347/COPPER

B. U-TUBE

C\&D. (TO BE DETERMINED BY 1 AUGUST 1969)

2. MIXED GAS REQUIREMENTS BY I NOVEMBER 1969

3. FINAL DILUENT FLUID CONDITIONS BY I AUGUST 1969

4. ONE OR TWO PORTS BY 1 AUGUST 1969

5. TURBINE INLET LINE INTERFACE BY I SEPTEMBER 1969

6. DILUENT EXTRACTED FROM DOME 


\section{NOZZLE-BLEED PORT INSERT}

DRB

ESSENTIALLY ALL INPUTS AVAILABLE

\section{PDR (DESIGN)}

S-31 STEADY STATE, NOMINAL CONDITIONS ONLY

5-38 MODAL ANALYSES \& PARTIAL FORCING FUNCTION ONLY

S-39 STATIC \& THERMAL LOADS ONLY

S-36 EXCLUSIVE OF VIBRATION AND VARIABILITY ANALYSES

R-202 FMA 75\% COMPLETE

PDR (DEMO)

R-202 FMA ONLY (NO QUANTITATNEE ARALYSES) 


\section{NOZZLE SKIRT DESIGN MATURITY}

FOR DRB $\quad 9-30-69$

GENERAL DESIGN ESTABLISHED W TH ANALYSIS ACCOUNTING FOR ALL FAILURE MODES EXCEPT VIBRATION AND ACOUSTIC LOADS.

DATA ITEMS SUPPORTING DESIGN IN MEMO FORM.

NO DESIGN ESTABLISHED FOR SKIRT FOR NSS DEVELOPMENT.

FOR PDR $\quad 11-24-69$

DATA ITEMS 75\% COMPLETE AND IN PRELIMINARY FORM.

RELIABILITY ANALYSIS UNAVAILABLE.

OPERATING MAP UNAVAILABLE.

NOTE: 6 MO. SLIP IF ENGINE $A_{e} / A_{t}$ OTHER THAN 100/1. 


\section{SKIRT EXTENSION DESIGN MATURITY}

FOR DRB 9-30-69

DESIGN CONCEPT SELECTED WITH SUPPORTING PRELIMINARY ANALYSIS EXCEPT FOR

FABRICABILITY VERIFICATION OF GRAPHITE COMPOSITE CONCEPT.

NO ACCOUNTABILITY FOR VIBRATION AND ACOUSTIC LOA DS.

FOR PDR $11-24-69$

GENERAL DESIGN ESTABLISHED WITH DATA ITEMS 50\% COMPLETE AND IN PRELIMINARY FORM

RELIABILITY ANALYSIS UNAVAILABLE.

OPERATING MAP UNAVAILABLE.

NOTE: 6 MO SLIP IF $A_{e} / A_{t}$ SELECTED IS OTHER 100// OR TURBINE EXHAUST GAS COOLED. 


\section{POISON WIRE REQUIREMENTS}

\section{PREFERRED OPERATION}

ASSUMPTIONS:

1. ENGINE ASSEMBLY, CHECKOUT AND ACCEPTANCE TESTING AT KSC.

2. REACTOR FULLY POISONED DURING HANDLING.

3. REMOVE ALL POISON WIRES FOR ENGINE ACCEPTANCE TESTING.

4. REINSERT CENTRAL POISON WIRES UPON COMPLETION OF ACCEPTANCE TESTING.

5. REMOVE CENTRAL POISON WIRES PRIOR TO LAUNCH FOR ALL MISSIONS. 


\section{POISON WIRE REQUIREMENTS}

\section{ALTERNATE OPERATION}

ASSUMPTIONS:

1. ACCEPTANCE TEST ENGINE AT MTF

2. REACTOR FULLY POISONED DURING HANDLING

3. REMOVE ALL POISON WIRES FOR ENGINE ACCEPTANCE TEST

4. REINSERT ALL POISON WIRES UPON COMPLETION OF ENGINE ACCEPTANCE TEST. (NOZZLE CAN BE REMOVED FOR INSTALLATION OF POISON WIRES.)

5. ASSEMBLE ENGINE, STAGE AND ASCENT SHELL AT MICHOUD AND PERFORM STAGE ACCEPTANCE TESTS

6. SHIP TO KSC BY BARGE

7. REMOVE PERIPHERAL POISON WIRES PRIOR TO VEHICLE ASSEMBLY

8. REMOVE CENTRAL POISON WIRES PRIOR TO LAUNCH FOR ALL MISSIONS 
POISON WIRE WITHDRAWAL/REINSERTION DEVICE

KNOWN:

1. NUMBER OF POISON WIRES CENTRAL AND PERIPHERAL (CLASSIFIED)

2. WITHDRAWAL RATE (WANL PRELIMINARY)

3. WITHDRAWAL FORCE (WANL PRELIMINARY)

UNKNOWN:

1. POISON WIRE FINAL CONFIGURATION (WANL)

2. POISON WIRE RETAINER DESIGN (WANL)

3. AGC/WANL INTERFACE

4. FINAL POISON WIRE OPERATIONAL REQUIREMENTS

ASSUMPTIONS: 1. WANL POISON WIRE CONFIGURATION WILL BE SAME AS 910 E 186

2. WANL RETAINER WILL BE AS SHOWN ON 929 F 109

3. POISON WIRE REQUIREMENTS ARE THOSE OUTLINED IN 23 MAY 1969 MEMO, SUBJECT: ACCEPTANCE OF NERVA ENGINE, FROM R. W. SCHROEDER TO W. O. WETMORE 
POISON WIRE WITHDRAWAL/REINSERTION DEVICE

DRB $\quad 9-30-69$

SELECTION OF A POISON WIRE WITHDRAWAL/REINSERTION CONCEPT.

WANL PARTICIPATION REQUIRED IN THE FOLLOWING ARE AS:

AGC WANL INTERFACE TRADE STUDY

WIRE WITHDRAWAL VELOCITY

WIRE WITHDRAWAL FORCE

WIRE RE TAINER SELECTION

ANALYTICAL WORK TO SUPPORT DESIGN SELECTION LIMITED TO EXPLORATION OF FEASIBILITY OF EACH CONFIGURATION, IDENTIFICATION OF PROBLEM AREAS \& SUGGESTED SOLUTIONS.

DATA ITEMS PREPARED WILL REFLECT PRELIMINARY NATURE OF THIS WORK.

PDR $11-24-69$

DETAILED STUDIES WILL ASSESS ENVIRONMENTAL CONDITIONS, THERMAL, STRESS, LOADS AND VIBRATION ANALYSIS, MATERIALS, RELIABILITY AND FABRICATION EVALUÁTION TO ESTABLISH THAT THE CA NDIDATE POISON WIRE WITHDRAWAL/REINSERTION DEVICE WILL PERFORM ALL OF THE SPECIFIED POISON WIRE REOUIREN TION WITH OTHER POISON WIRE DEVICES.

DATA ITEMS WILL BE COMPILED， DOCUMENTED TO THE EKTENT OF $\$ .5 \%$ COMPLETION FOR PDR REVIEW. 


\section{NERVA LINES \\ GROSS INFORMATION FOR PDR DECEMBER 1969}

KNOWN

ALL KEY OPERATING PARAMETERS RELATED TO STEADY STATE FLOW CONDITIONS.

UNKNOWN

ROUTING WITH DISC SHIELD

LINE DIAMETERS VERSUS NUMBER OF LINES (T.S.)

ONE VERSUS TWO TIL, DIL (T.S.)

ONE VERSUS 24 PDL (T.S.)

EXTREME OPERATING CONDITIONS INCLUDING HEAT GENERATION RATES.

CONFIGURATION OF ARTICULATED ME MBERS (T.S.)

LOCATION OF ARTICULATED MEMBERS (T.S.)

ASSUMP TIONS

TWO TPA, SAME CONFIGURATION, SAME LOADS

SIX TURBINE VALVES RIGIDLY SUPPORTED

DISC SHIELD DIAMETER AND STATION AS DWG 1136339 


\section{NERVA LINES}

CONTENT FOR DRB

LAYOUTS OF ALL REFERENCE ENGINE LINES

CALCULATED PRESSURE DROP, FLANGE LOADS, AND WEIGHTS FOR REFERENCE ENGINE LINES

LAYOUTS OF ONE OR TWO ALTERNATE ROUTING/BELLOWS CONCEPTS

DIAMETERS DEFINED

CONTENT FOR PDR

PARTIAL COMPLETION OF S-54 DESIGN TRADE STUDIES

. CIME SECONDATRGAMMA EMISSION

LINE ROUTING AND FLEXIBILITY

LINE MATERIAL SELECTION

LINE JOINT SELECTION

LAYOUTS OF 3 OR MORE ALTERNATES

LISTING OF MAXIMUM FLANGE LOADS \& PRESSURE DROPS BASED ON ANALYSIS OF 3 ALTERNATES

MODE OF FAILURE ANALYSES, BUT NO RELIABILITY ANALYSES 
BACKUP WIEWGRAPHS FOR

HWT MTE 6-7-54

669-309 thIN 669-307 MASTER

VELLANG HELO RY E. LATIN 


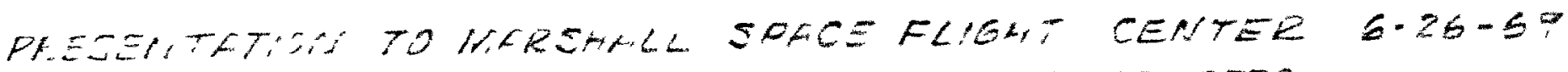

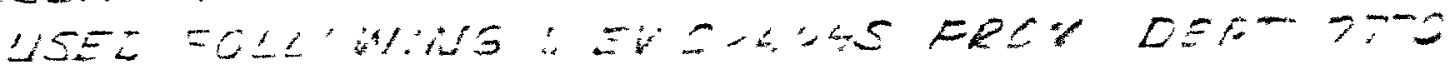

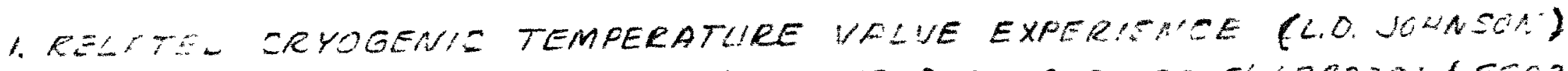

2. XE 5 INCH INTERNAL GIMEALL BELLOWS.OUAL. RESULTS SINBBC3CI I S8C3O5:

3. - CONT. OF 2

4 CONT UF 2

5 -X.ENGINE S" GMBALLED BELLOWS

$6 X E S^{\prime \prime}$ LIIE SEGMENT 
669-308 PHOEBUS COAXIAL INUECTOR VIEGRAPH MADE FROM PHOTO 
aGc:ADO

\section{OXYGEN/HYDROGEN TEST SUMMARY}

MR

$1.0-15.0$

$4.3-9.6$

$4.3-.75$
$P_{c}$

$25-100$

$100-350$

$350-540$
THRUST

$7 K-30 K$

$30 K-120 K$

$120 \mathrm{~K}-170 \mathrm{~K}$
TESTS

18

19

TOTAL $\frac{6}{43}$

IN ADDITION WE HAD:

(1) 19 SPARK IGNITION TESTS RANGING BETWEEN $1.7-8.3$ MR AND $295-429 \mathrm{P}_{\mathrm{c}}$.

(2) 18 MISCELLANEOUS ENGINE AND THRUST CHAMBER TESTS RANGING BETWEEN $3.4-8.9$ MR AND $120-435 P_{C}$. 
TECHNOLOGY NOZZLE TEST DATA

SN 027 CHEMICAL SIMULATION

\begin{tabular}{|c|c|c|}
\hline DURATION, SEC & CHAMBER PRESSURE, PSIA & MIXTURE RATIO \\
\hline 15.1 & 674 & 5.8 \\
\hline 1.7 & 437 & 1.5 \\
\hline 6.5 & 721 & 4.5 \\
\hline 3.5 & 661 & 355 \\
\hline 5.8 & 645 & 6.2 \\
\hline 20.8 & 736 & 6.0 \\
\hline 20.5 & 789 & 5.8 \\
\hline 12.0 & 801 & 5.7 \\
\hline 20.4 & 801 & 5.7 \\
\hline
\end{tabular}


TECHNOLOGY NOZZLE TEST DATA

SN 021 CHEMICAL SIMULATION

\begin{tabular}{|c|c|c|c|c|}
\hline \multirow{2}{*}{ DURATION, SEC } & \multicolumn{2}{|c|}{ CHAMBER PRESSURE, PSIA } & \multicolumn{2}{c|}{ MIXTURE RATIO } \\
\cline { 2 - 5 } & TARGET & ACTUAL & TARGET & ACTUAL \\
\hline 8.3 & 560 & 605 & 5.00 & 8.28 \\
\hline 6.2 & 650 & 662 & 5.25 & 5.01 \\
\hline 13.4 & 650 & 654 & 4.50 & 4.23 \\
\hline 37.1 & 650 & 647 & 4.50 & 4.22 \\
\hline 15.3 & 650 & 640 & 4.60 & 4.53 \\
\hline 26.0 & 650 & 645 & 4.60 & 4.72 \\
\hline 25.5 & 650 & 638 & 4.60 & 4.72 \\
\hline 25.4 & 650 & 647 & 4.60 & 4.82 \\
\hline 25.6 & 650 & 649 & 4.60 & 4.53 \\
\hline 68.4 & 650 & 652 & 4.60 & 4.59 \\
\hline
\end{tabular}




\section{AGe.:ND}

SUMMARY OF NOZZLE REACTOR TESTS

\begin{tabular}{|l|c|c|c|c|c|}
\hline $\begin{array}{c}\text { REACTOR } \\
\text { TEST }\end{array}$ & $\begin{array}{c}\text { NOZZLE } \\
\text { S/N }\end{array}$ & $\begin{array}{c}\text { MAX NOZ. } \\
\text { TEMP } \\
\text { (OR) }\end{array}$ & $\begin{array}{c}\text { MAX NOZ. } \\
\text { PRESS. } \\
\text { (PSIA) }\end{array}$ & $\begin{array}{c}\text { DUR. AT } \\
\text { MAX POWER } \\
\text { (MIN.) }\end{array}$ & $\begin{array}{c}\text { NO. OF RESTARTS } \\
\text { TO LOWER POWER }\end{array}$ \\
\hline NRX A3 & 22 & 3800 & 570 & 16 & 4 \\
\hline NRX A5 & 24 & 4100 & 570 & 31 & 1 \\
\hline NRX/EST & 31 & 4100 & 590 & 60 & 0 \\
\hline XE & 28 & 4100 & 567 & 30 & 5 \\
\hline
\end{tabular}


PHOEBUS 2 NOZZLE TEST DATA

CHEMICAL SIMULATION

\begin{tabular}{|c|c|c|c|c|}
\hline DURATION, SEC & \multicolumn{2}{|c|}{ CHAMBER PRESSURE, PSIA } & \multicolumn{2}{|c|}{ MIXTURE RATIO } \\
\hline & TARGET & ACTUAL & TARGET & ACTUAL \\
\hline 1.9 & 685 & 620 & 5.5 & 5.8 \\
\hline 1.9 & & 590 & & 5.9 \\
\hline 2.1 & & 665 & & 5.3 \\
\hline 4.0 & & 690 & & 5.3 \\
\hline 5.7 & & 685 & & 5.3 \\
\hline 15.4 & & 690 & & 5.4 \\
\hline 15.2 & & 685 & & 5.4 \\
\hline 15.8 & & 687 & & 5.5 \\
\hline 14.7 & & 686 & & 5.4 \\
\hline 14.6 & & 684 & & 5.4 \\
\hline 15.7 & & 686 & & 5.4 \\
\hline 14.9 & & 687 & & 5.4 \\
\hline 12.1 & 685 & 686 & 5.5 & 5.4 \\
\hline
\end{tabular}




\section{Ace्.10}

CONVERGENT SECTION-CONTROLLED DILUENT-110 ELLIPTICAL HOLES-SLEEVE FEED

(MATERIAL: CRES 347)
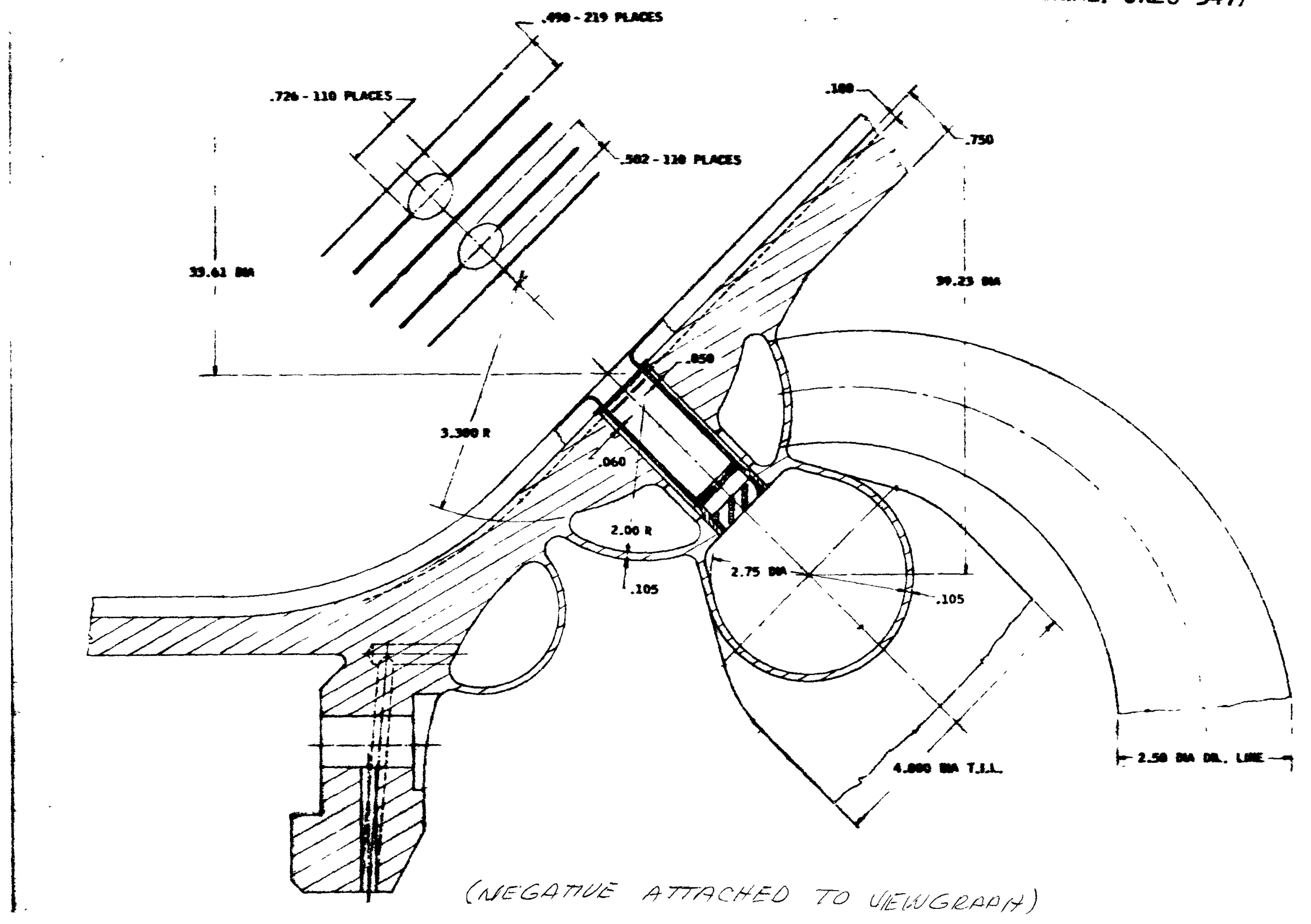


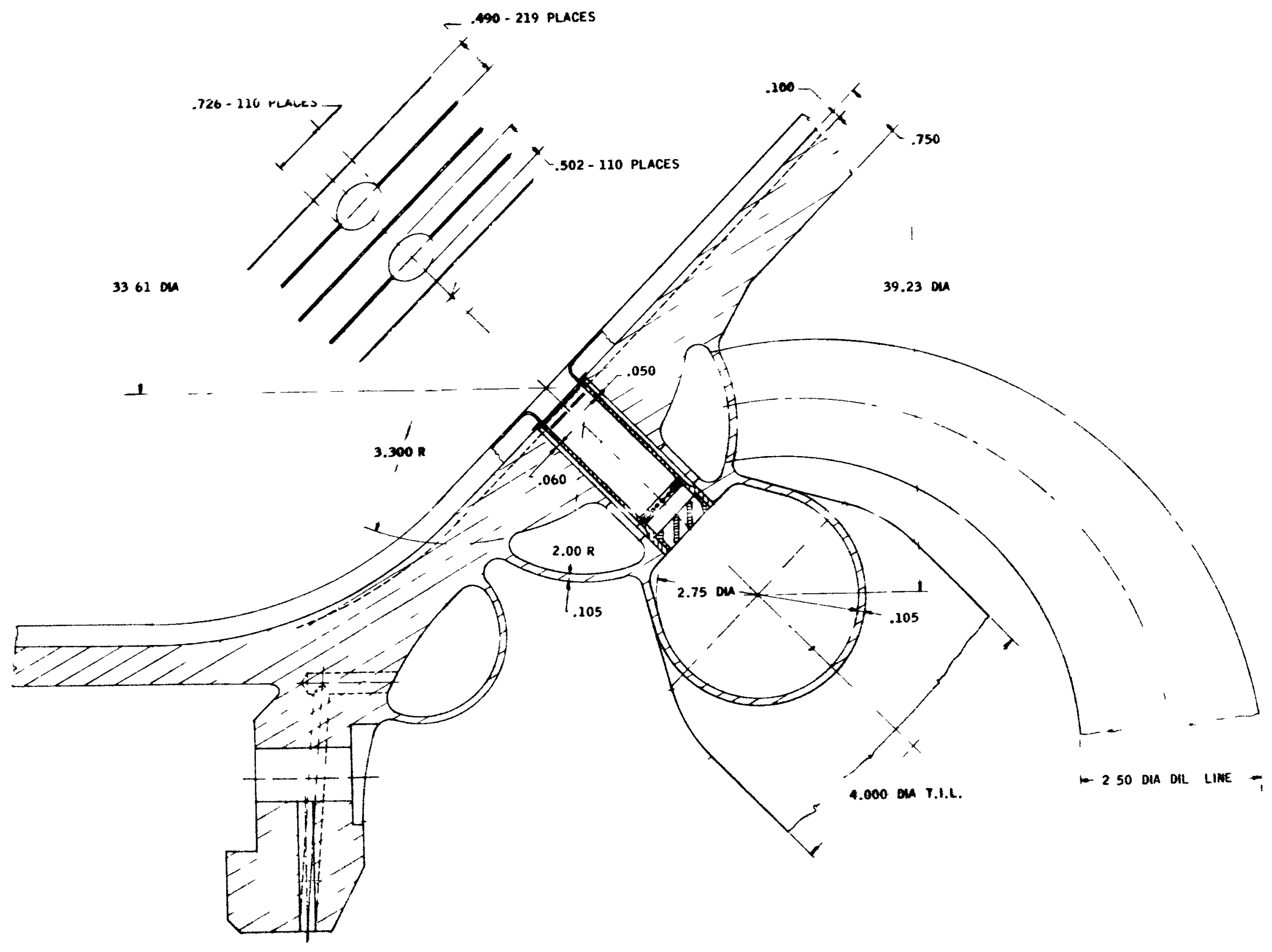


A6C.A.110 CONTROL SYSTEM FOR PHOEBUS I CHEMICAL SIMULATION TESTS

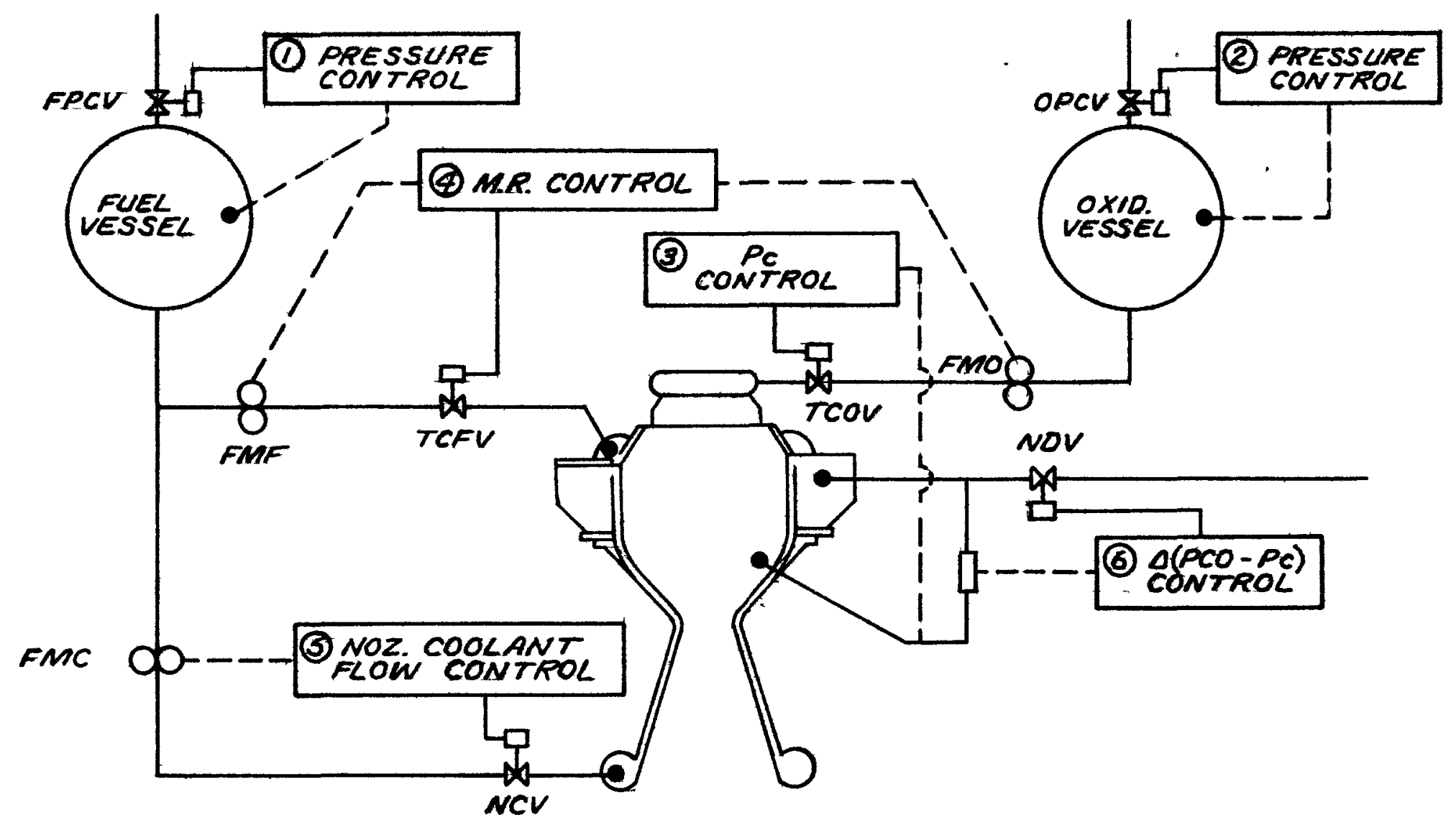




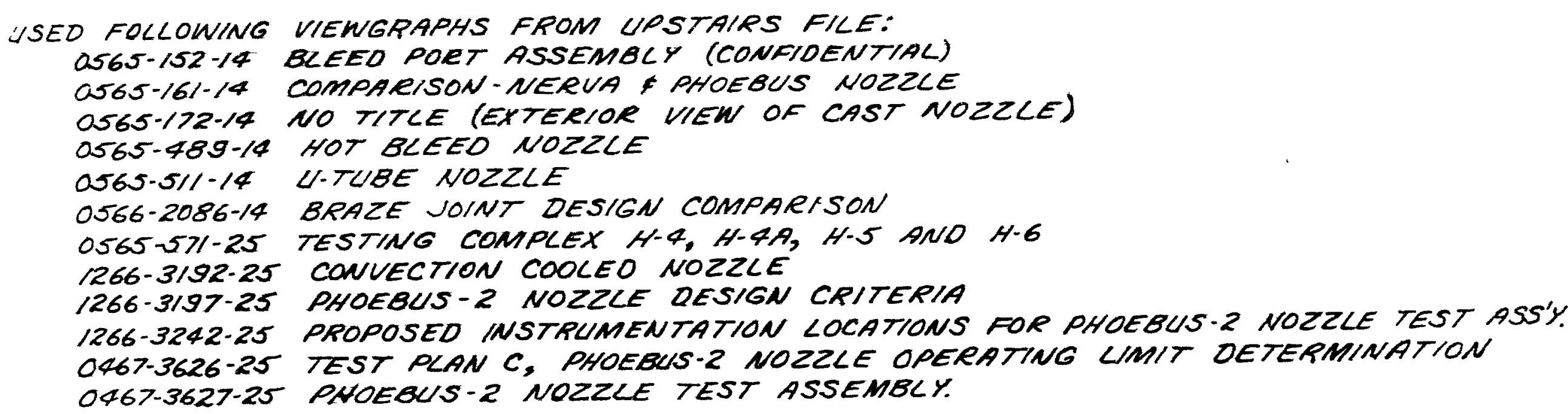

